University of Louisville

ThinkIR: The University of Louisville's Institutional Repository

Electronic Theses and Dissertations

$12-2019$

\title{
Electrospun fibers and nanoparticles for the prevention of sexually transmitted infections.
}

Kevin Michael Tyo

University of Louisville

Follow this and additional works at: https://ir.library.louisville.edu/etd

Part of the Nanomedicine Commons

\section{Recommended Citation}

Tyo, Kevin Michael, "Electrospun fibers and nanoparticles for the prevention of sexually transmitted infections." (2019). Electronic Theses and Dissertations. Paper 3344.

https://doi.org/10.18297/etd/3344

This Doctoral Dissertation is brought to you for free and open access by ThinkIR: The University of Louisville's Institutional Repository. It has been accepted for inclusion in Electronic Theses and Dissertations by an authorized administrator of ThinkIR: The University of Louisville's Institutional Repository. This title appears here courtesy of the author, who has retained all other copyrights. For more information, please contact thinkir@louisville.edu. 


\title{
ELECTROSPUN FIBERS AND NANOPARTICLES FOR THE PREVENTION OF SEXUALLY TRANSMITTED INFECTIONS
}

\author{
By \\ Kevin Michael Tyo \\ BS, Virginia Tech, 2010 \\ MS, University of Louisville, 2016

\begin{abstract}
A Dissertation
Submitted to the Faculty of the

in Partial Fulfillment of the Requirements

for the Degree of

Doctor of Philosophy

in Pharmacology and Toxicology
Department of Pharmacology and Toxicology
University of Louisville
Louisville, Kentucky \\ School of Medicine of the University of Louisville
}

December 2019 
Copyright 2019 by Kevin Michael Tyo

All rights reserved 



\title{
ELECTROSPUN FIBERS AND NANOPARTICLES FOR THE PREVENTION OF SEXUALLY TRANSMITTED INFECTIONS
}

\author{
By \\ Kevin Michael Tyo \\ BS, Virginia Tech, 2010 \\ MS, University of Louisville, 2016
}

A Dissertation Approved on

September 24, 2019

by the following Dissertation Committee:

Dr. Jill M. Steinbach-Rankins

Dr. Hermann B. Frieboes

Dr. Joshua L. Fuqua

Dr. Nobuyuki Matoba

Dr. Kenneth E. Palmer 


\section{DEDICATION}

I dedicate this Ph.D. dissertation to my parents, my family, and friends, whom I am eternally grateful for their love, and their continued support. 


\section{ACKNOWLEDGEMENTS}

I have had the pleasure and blessed fortune of having many mentors in my life. Without them, I would not be the person I am today.

First and foremost, I would like to thank Dr. Jill Steinbach-Rankins for her guidance as my Ph.D. mentor. During my tenure at the University of Louisville, she has always supported me in my scientific endeavors, as well as challenging me to grow as a student and as a scientist. I know she has spent countless hours to accomplish this task. I am in awe of her ability to surmount any problem and to graciously manage multiple projects. I first joined her laboratory based on solely on her research in delivery platforms. However, as time went on, I began to appreciate working in the vibrant laboratory environment that Dr. SteinbachRankins has labored so extensively to create. I'm eternally grateful that I have been given the opportunity to work with such a hardworking, intellectual, considerate, and forgiving human being.

I would also like to thank the other members of my dissertation committee, Dr. Frieboes, Dr. Fuqua, Dr. Matoba, and Dr. Palmer for agreeing to lend their expertise, and assistance during the duration of my Ph.D. studies. Along with Dr. Steinbach-Rankins, I could not have asked for a greater group of experts for my committee. 
I would also like to thank all the members of the Steinbach-Rankins, past and present. Specifically, I would like to thank Farnaz Minooei, Keegan Curry, Longyun Zhang, Kyle Vuong, Steven Winter, Sarah NeCamp, Lee Sims, and Danial Malik for both their kindness and friendship.

I would also like to thank my colleagues from industry Mr. Bill Adams, Dr. Jay Pierotti, and Derek Burton. I am grateful for their friendship and their guidance prior to my tenure at the University of Louisville.

Lastly, I would like to acknowledge my parents, Michael and Teresa Tyo who have supported me from the very beginning. Despite all the tough times we have experienced, they have never wavered in their support for me. I am extremely thankful that I have such loving parents. 


\begin{abstract}
ELECTROSPUN FIBERS AND NANOPARTICLES FOR THE PREVENTION OF SEXUALLY TRANSMITTED INFECTIONS

Kevin Michael Tyo
\end{abstract}

September 24, 2019

Human immunodeficiency virus-1 (HIV-1) and herpes simplex virus 2 (HSV-2) affect hundreds of millions of people worldwide, with women disproportionately impacted by these infections. Currently, only oral pre-exposure prophylaxis (PrEP) is approved specifically for the prevention of HIV-1, but is challenged with adverse side effects associated with long-term use. Topical delivery platforms, such as gels and films, deliver agents directly to the female reproductive tract, but are limited in providing transient-release. The technology of polymeric electrospun fibers may serve as alternative topical delivery platform to the female reproductive tract. In these studies, we fabricated electrospun fibers comprised of different polymers or polymer blends that possess different physical attributes and fiber architectures. The goal was to provide sustained-release of agents such as the antiretroviral tenofovir disoproxil fumarate (TDF) and the antiviral lectin, Griffithsin (GRFT). We hypothesized that these delivery platforms would prevent HIV-1 and HSV-2 infections, while retaining the safety and biocompatibility of free agent. To determine the amount of GRFT loading and release from fiber formulations, ELISA 
was conducted, whereas TDF quantification was performed using absorbance measurements. Next, the in vitro efficacy of composites was assessed in HIV-1 and HSV-2 infectivity assays. From these initial results, multilayered fiber composites, free NPs, and hydrophilic fibers were tested for safety and antiviral efficacy within a murine model. Animal studies were conducted using 5-week-old female BALB/c mice, histology and cytokine expression were evaluated from mouse reproductive tracts and vaginal lavages collected 24 and $72 \mathrm{hr}$ following platform administration. In parallel experiments, mice were administered fibers, followed by a single challenge 4 or 24 hr later with HSV-2 (LD90). Viral progression was monitored for 14 days post viral challenge to evaluate potential infection. Statistical significance for all studies was determined using one-way ANOVA with Bonferroni post hoc test $(p<0.05)$, while log-ranked post hoc tests were used for antiviral efficacy studies. Future studies will consider encapsulation of multiple antiviral compounds to provide synergetic protection against infection.

Chapters included in this dissertation represent papers that have been or will be submitted, which may result in duplicate descriptions across chapters; however, these have been provided for the sake of completeness. CHAPTER 1 contains material that has been published in Pharmaceutics. CHAPTER 2 was published in the International Journal of Pharmaceutics, whereas CHAPTER 3 was published in the European Journal of Pharmaceutics and Biopharmaceutics. Finally, the appendices section contains material published in the Journal of Visualized Experiments. CHAPTERS 4 and 5 are undergoing preparation for submission. 
TABLE OF CONTENTS

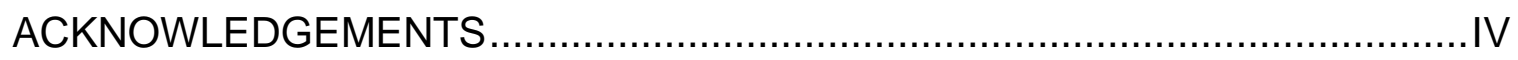

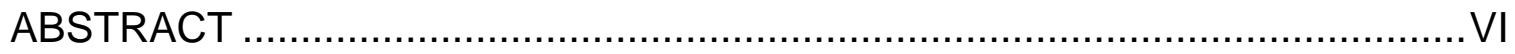

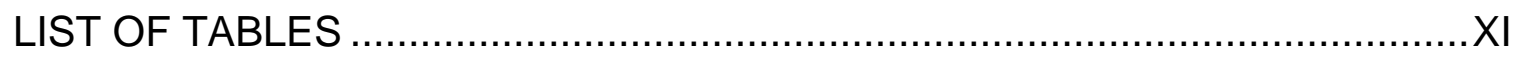

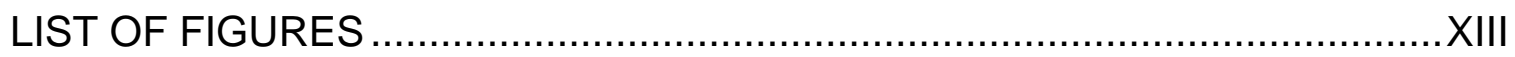

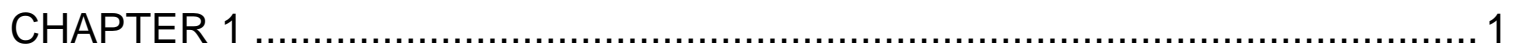

BACKGROUND, SIGNIFICANCE, AND RELATING ADVANCED ELECTROSPUN FIBER ARCHITECTURES TO THE TEMPORAL RELEASE OF ACtIVE AGENTS tO MEET the NEEDS OF NEXT-GENERATION INTRAVAGINAL DELIVERY APPLICATIONS ........................................... 1

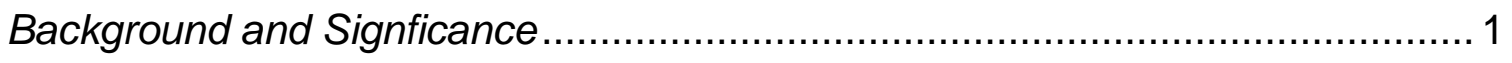

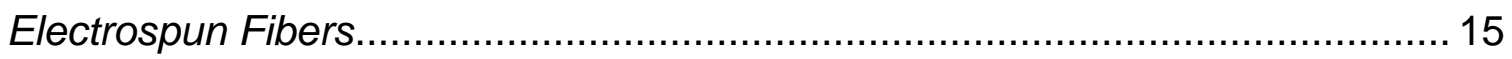

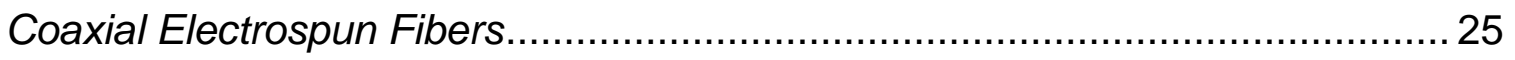

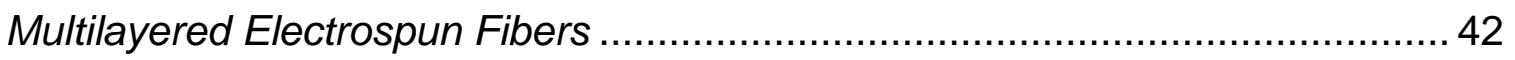

Composite Nanoparticle-Fiber Delivery Vehicles.......................................... 52

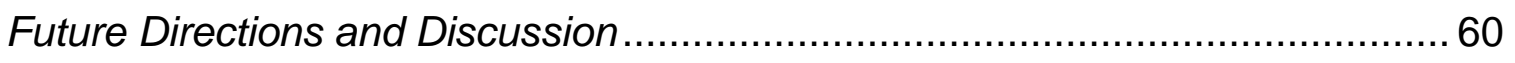

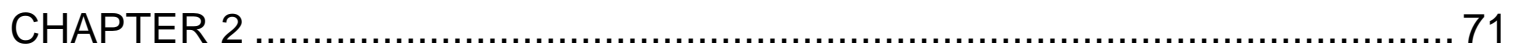


MULTIPURPOSE TENOFOVIR DISOPROXIL FUMARATE ELECTROSPUN FIBERS FOR THE PREVENTION OF HIV-1 AND HSV-2 INFECTIONS IN VITRO. .71

Introduction. 71

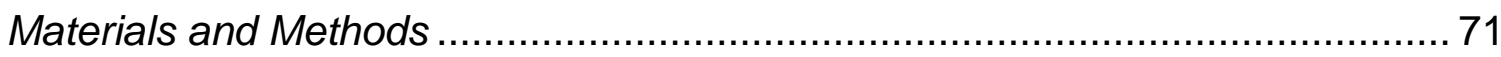

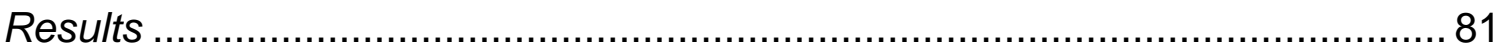

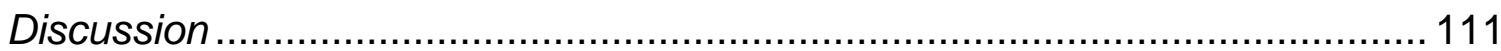

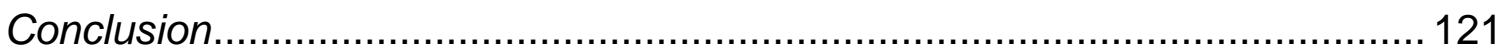

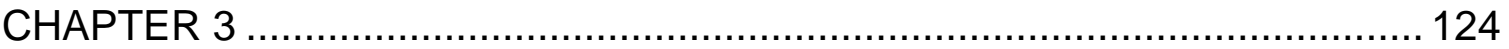

PH-RESPONSIVE DELIVERY OF GRIFFITHSIN FROM ELECTROSPUN

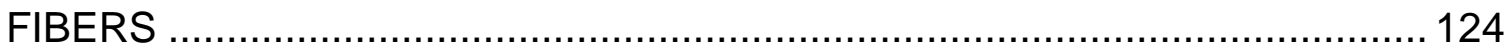

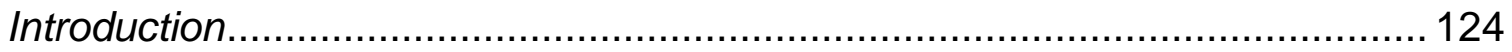

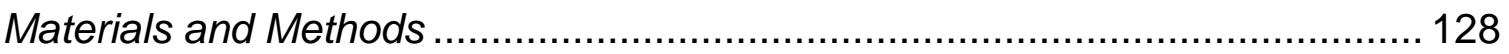

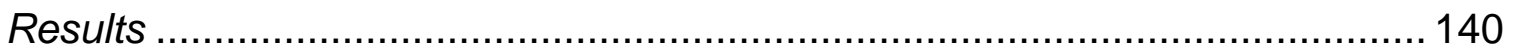

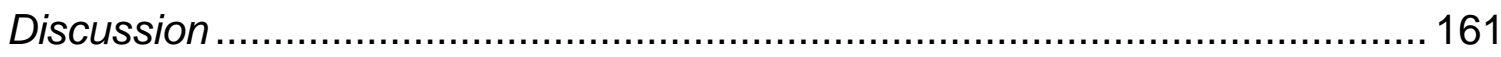

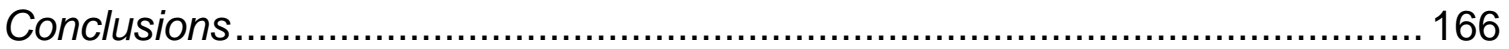

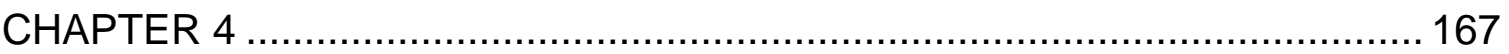

RAPID RELEASE GRIFFITHSIN ELECTROSPUN FIBERS FOR USE AGAINST

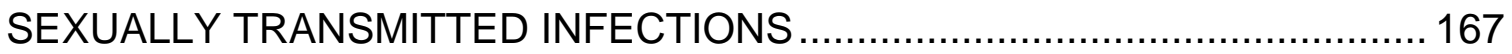

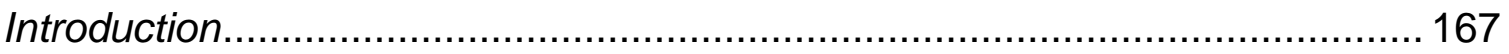

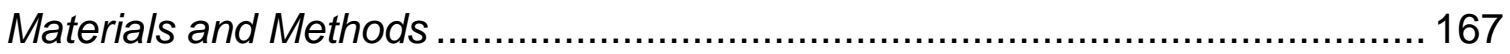

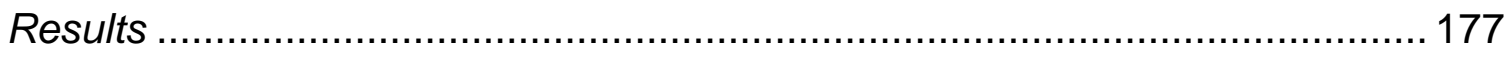

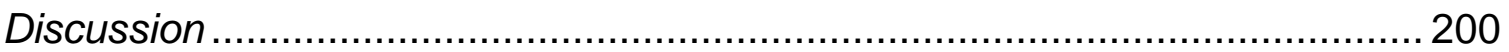


CHAPTER 5

MULTIPURPOSE GRIFFITHSIN NANOPARTICLE-ELECTROSPUN FIBER COMPOSITES AGAINST HIV-1 AND HSV-2 INFECTIONS .......................... 208

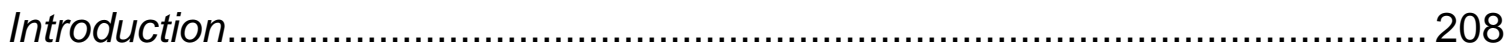

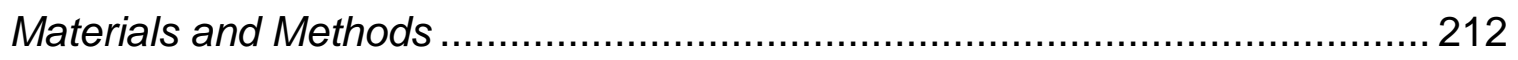

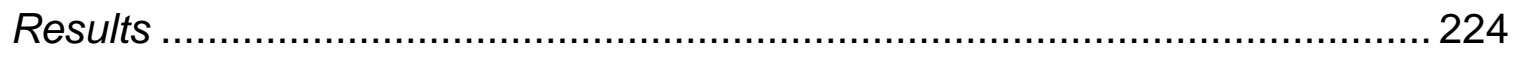

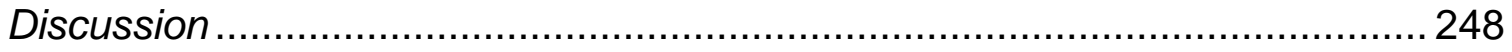

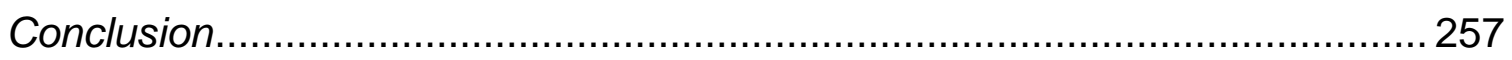

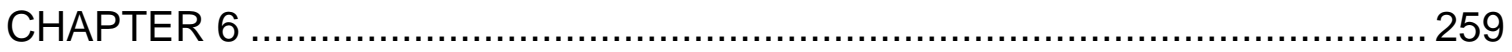

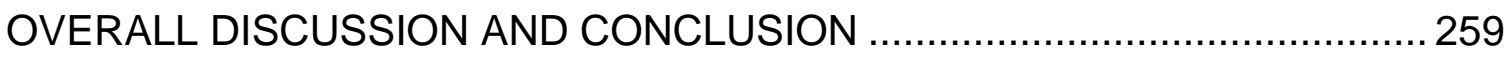

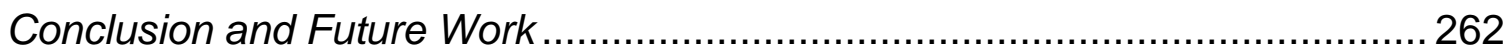

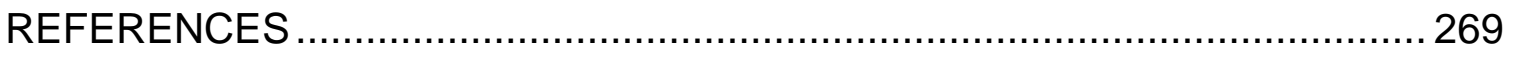

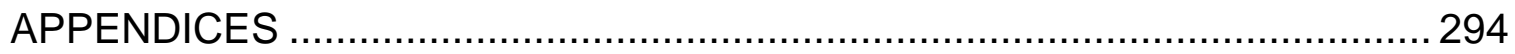

FABRICATION AND CHARACTERIZATION OF GRIFFITHSIN-MODIFIED FIBER SCAFFOLDS FOR PREVENTION OF SEXUALLY TRANSMITTED

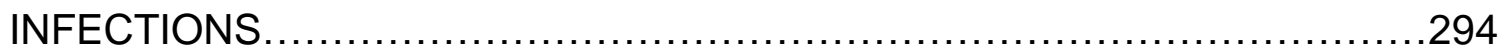

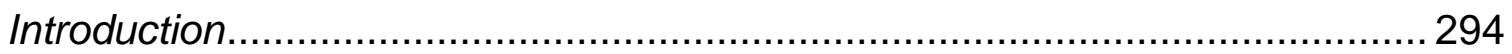

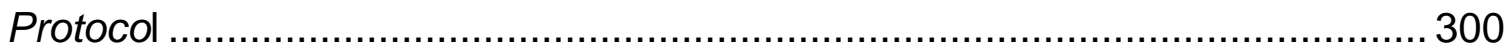

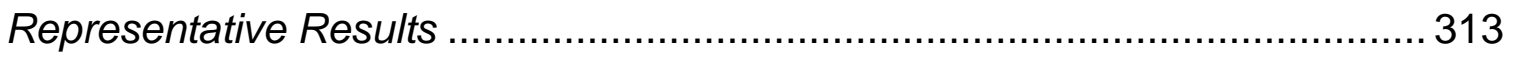

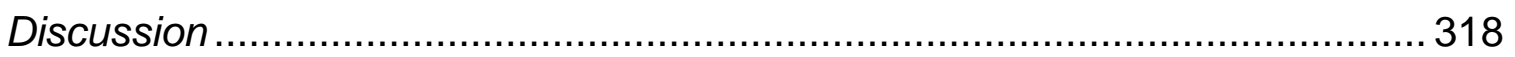

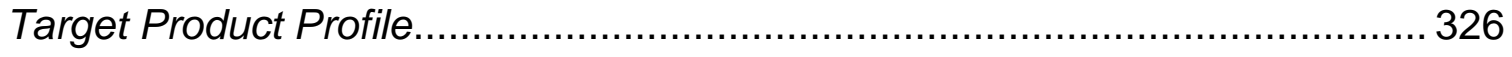

LIST OF ABBREVIATIONS ..................................................

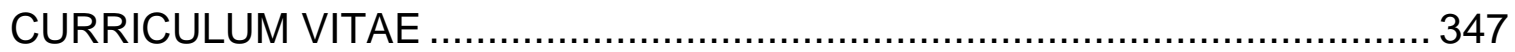




\section{LIST OF TABLES}

Table 2.1 Diameters of electrospun fibers. Blank and TDF fibers were

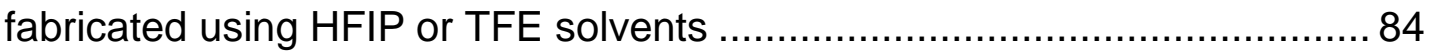
Table 2.2 Quantification of TDF fiber loading and encapsulation efficiency. 91 Table 2.3 The $\mathrm{IC}_{50}$ S of PLGA and PLCL fibers (against HIV-1) after administration of the 1 and $24 \mathrm{~h}$ release eluates. ..................................... 97 Table 2.4 The $\mathrm{IC}_{50}$ S of PLGA and PLCL fibers (against HIV-1) after

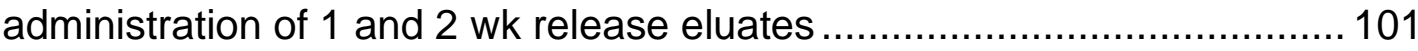
Table 2.5 The IC50S of PLGA and PLCL fibers (against HSV-2) after administration of the 1 and $24 \mathrm{hr}$ release eluates. solvents ..................... 105 Table $3.1 \mathrm{pH}$-responsive fiber diameters measured from SEM images ..... 144 Table 3.2 GRFT loading and encapsulation efficiency based on formulation

Supplemental Table 4.1 Fiber diameters as a function of polymer formulation

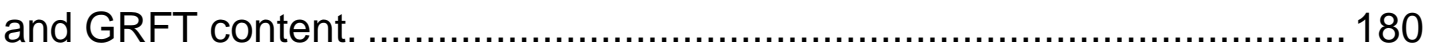

Table 4.1 IC 50 values of in vitro HIV-1 and HSV-2 infection assays........... 185 Table 4.2 GRFT doses administered in fibers or gel for in vivo HSV-2 infectivity studies 189

Table 5.1 Griffithsin loading in mPEG-PLGA and PLGA NPs .................. 228 
Table 5.2 Coumarin-6 NP loading in PEO, PVA, and PVP NP-EF composites 232

Table 5.3 Dosing regimen of GRFT NPs and NP-EF composites for in vivo studies. 242

Table A.1 GRFT Electrospun Fibers Target Product Profile. 326

Table A.2 GRFT Nanoparticle Target Product Profile............................... 335 


\section{LIST OF FIGURES}

Figure 1.1 Map depicting the total number of people living with HIV-1 infection worldwide.

Figure 1.2 Schematic depicting the structure and specific layers of the vaginal mucosa that can act as a barrier to active agent transport (not to scale) ..... 12 Figure 1.3 Schematic depicting examples of transient, short-term, and

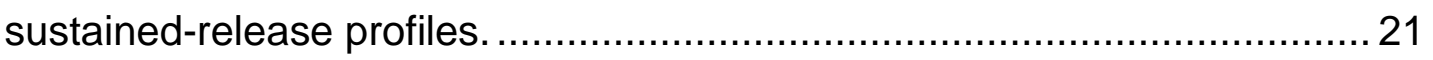

Figure 1.4 Schematic of different electrospun fiber composites.................. 24 Figure 1.5 Schematic of anticipated release profiles from different coaxial fiber

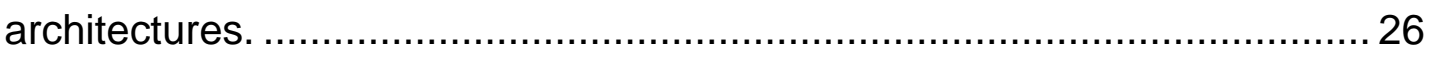
Figure 1.6 Schematic of anticipated active agent release from multilayered fibers 45

Figure 1.7 Schematic of electrospun nanoparticle-fiber composites that integrate coaxial and multilayered fiber architectures ............................... 58

Figure 2.1 SEM images of blank PLGA and PLCL fibers electrospun using different solvents. 83

Figure 2.2 SEM images of PLGA and PLCL fibers prepared with increasing concentrations of TDF, using HFIP as the solvent ................................... 85 Figure 2.3 Average diameters of electrospun fibers measured from SEM images, using ImageJ 87 
Supplemental Figure 2.1 HPLC chromatogram of (A) a standard containing pure TDF and TFV, (B) 20\% PLGA TDF EF eluate, and (C) 20\% PLCL TDF EF eluate. 89

Figure 2.4 Release profiles of TDF from 1, 10, and 20\% TDF PLGA and PLCL fibers in SVF 93 Figure 2.5 PLGA and PLCL fiber eluates inhibit HIV-1 infection in vitro after 1 and $24 \mathrm{~h}$, and 1 and $2 \mathrm{wk}$ of release 95 Figure 2.6 Both PLGA and PLCL fiber release eluates prevent HSV-2 infection in vitro 103

Figure 2.7 Cytotoxicity assessed via MTT assay 107 Figure 2.8 EpiVaginal cytotoxicity was assessed via H\&E staining (A) and LDH assay (B) 108

Figure 2.9 Cytokine expression from EpiVaginal studies after (A) 48 and (B) $72 \mathrm{~h}$ administration of fibers 110

Figure 3.1 Schematic of mPEG-PLGA and PBA-co-PAA co-polymers and the fiber fabrication process. 132

Supplemental Figure 3.1 NMR Spectrograph of GRFT Fibers 138 Figure 3.2 SEM images of (A) PLGA and (B) mPEG-PLGA electrospun polymer fibers that incorporate GRFT. 142

Figure 3.3 SEM images of different $\mathrm{pH}$-responsive electrospun fiber blends that incorporate GRFT 143

Figure 3.4 In vitro release profiles of GRFT from mPEG-PLGA fibers 148 
Figure 3.5 In vitro $\mathrm{pH}$-responsive release profiles of GRFT from different PLGA:PBA-co-PAA blended fiber formulations, each theoretically loaded with $30 \mu \mathrm{g}$ GRFT/mg polymer. 150

Figure 3.6 In vitro pH-responsive release profiles of GRFT from mPEGPLGA:PBA-co-PAA (90:10, w/w) fibers. 154

Figure 3.7 HIV-1 inhibition assays were conducted to assess the functional activity of extracted GRFT after electrospinning into fibers, relative to free GRFT 156

Figure 3.8 HIV-1 inhibition assays were conducted to assess the antiviral activity of GRFT mPEG-PLGA:PBA-co-PAA (90:10) fiber release eluates against HIV-1 infection. 158

Figure 3.9 In vitro safety evaluation of mPEG-PLGA:PBA-co-PAA (90:10) fibers on cervicovaginal cell viability.. 160

Figure 4.1 Scanning electron microscopy (SEM) images of (A-C) blank, (D-F) 1\% w/w GRFT, and (G-I) 10\% w/w GRFT fibers. 179

Figure 4.2 GRFT loading in different hydrophilic fiber formulations 182 Figure 4.3 GRFT fibers demonstrate complete protection against in vitro HIV1 and HSV-2 infections. 184

Figure 4.4 The cytotoxicity of PEO, PVA, and PVP fibers administered to vaginal VK2/E6E7, Ect1/E6E7, and End1/E6E7 cell lines for (A) 24, (B) 48, and (C) $72 \mathrm{hr}$ was assessed using the MTT assay. 187

Figure 4.5 Schematic timetable and Kaplan-Meier survival curves of in vivo HSV-2 efficacy study 190 
Figure 4.6 Griffithsin fibers protect mice against HSV-2 infection 193

Figure 4.7 The in vivo safety of rapid release fibers was assessed by intravaginally administering fibers for 24 and $72 \mathrm{hr}$. 195 Supplemental Figure 4.1 Images of H\&E stained tissue outliers 196 Figure 4.8 Cytokine expression from extracted murine tissue and vaginal lavages collected (A, B) 24 and (C, D) $72 \mathrm{hr}$ after fiber administration. ..... 199 Figure 5.1 Schematic depicting the incorporation of NPs within hydrophilic fibers to create multilayered NP-EF composites 211

Figure 5.2 Scanning electron microscopy images of (A) blank, (B) 50, (C)100, and (D) $200 \mu \mathrm{g} / \mathrm{mg}$ GRFT PLGA NPs, and (E) blank, (F) 50, (G) 100, and (H) $200 \mu \mathrm{g} / \mathrm{mg}$ GRFT mPEG-PLGA NPs 225

Supplemental Figure 5.1 Characterization of the average diameter of blank and GRFT NPs 226

Figure 5.3 The cumulative release of GRFT from mPEG-PLGA and PLGA NPs shown as $(A)$ total GRFT release or $(B)$ the percent of total loading .......... 230 Figure 5.4 Scanning electron microscopy images of GRFT NP-EF composites taken from $(A)$ inner PEO fibers $(5 \% \mathrm{w} / \mathrm{w})$ that incorporate $20 \% \mathrm{w} / \mathrm{w}$ blank mPEG-PLGA NPs, (B) outer layer PCL fibers, and (C) a cross-sectional image of an NP-EF composite 234

Figure 5.5 Cumulative release of GRFT from multilayered GRFT NP-EF composites 236

Figure 5.6 Results from in vitro HIV-1 inhibition and MTT toxicity assays.. 238 
Figure 5.7 Schematic, timetable, and results of in vivo HSV-2 efficacy study 240

Figure 5.8 The safety of NPs and NP-EF composites was assessed by administering platforms to murine reproductive tracts for 24 and $72 \mathrm{hr}$..... 245 Supplemental Figure 5.2 The cumulative release of GRFT from multilayered fibers with different outer layer thicknesses shown as (A) total GRFT release

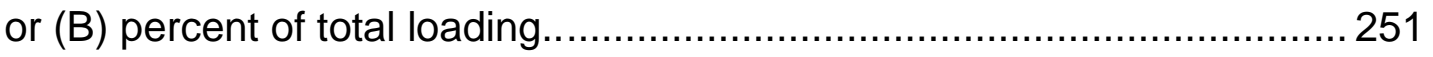

Figure A.1 The macroscale morphology of electrospun fibers .................. 296

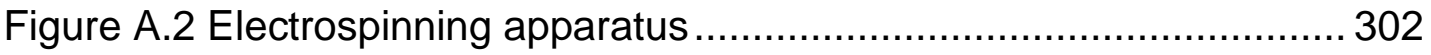

Figure A.3 Schematic of EF modification with GRFT using EDC-NHS

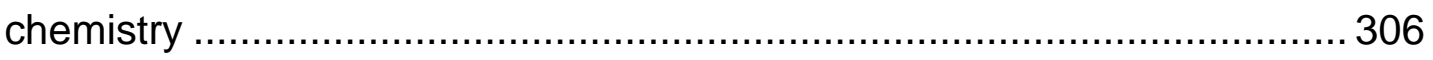

Figure A.4 Schematic illustrating GRFT quantification using ELISA .......... 311

Figure A.5 Effects of solvent choice on PLGA EF morphology .................. 314 Figure A.6 SEM images and fiber diameters of bare and GRFT-modified EFs 315

Figure A.7 Quantity of GRFT conjugated to and desorbed from GRFT-modified EF 317 


\title{
CHAPTER 1
}

\author{
BACKGROUND, SIGNIFICANCE, AND RELATING ADVANCED \\ ELECTROSPUN FIBER ARCHITECTURES TO THE TEMPORAL RELEASE OF \\ ACTIVE AGENTS TO MEET THE NEEDS OF NEXT-GENERATION \\ INTRAVAGINAL DELIVERY APPLICATIONS
}

\section{Background and Significance}

Sexually transmitted infections (STIs) represent a global health challenge, with over one million new cases reported daily. Currently, over 36 and 500 million people worldwide live with human immunodeficiency virus-1 (HIV-1) and herpes simplex virus type-2 (HSV-2) respectively ${ }^{1}$. Due to the socioeconomic conditions within parts of the developing world, women are disproportionately affected by these infections ${ }^{1}$. Furthermore, HSV-2 infection has been shown to significantly enhance the risk of HIV-1 acquisition by as much as 2 to 4 -fold ${ }^{2,3}$. Additionally, studies have shown that co-infected individuals harbor higher viral loads relative to individuals with only one infection type ${ }^{3}$. Despite decades of research, there is currently no cure for either HIV-1 or HSV-2, and infection rates among specific demographics remain high ${ }^{4,5}$.

The HIV-1 pandemic is a relatively recent phenomena, with the first confirmed cases of infections originating in the Democratic Republic of the Congo 
during the late $1950 s^{6}$. Human contraction of HIV-1 is believed to have originated from cross-species contamination of simian immunodeficiency viruses (SIVs), which normally infect primates. The first known cases of HIV-1 infection in the United States were observed in 1981, and were followed by the designation Acquired Immune Deficiency Syndrome (AIDS) in 1983, with the isolation of virus confirming its role in causing the disease. The virus itself, can be transmitted through homo- and heterosexual intercourse, mucosal layer exposure, or bloodto-blood transmission? ${ }^{7}$. To date, over 36 million people worldwide live with HIV-1 infection, with women in the developing world bearing the highest burden of infection (Figure 1.1). 


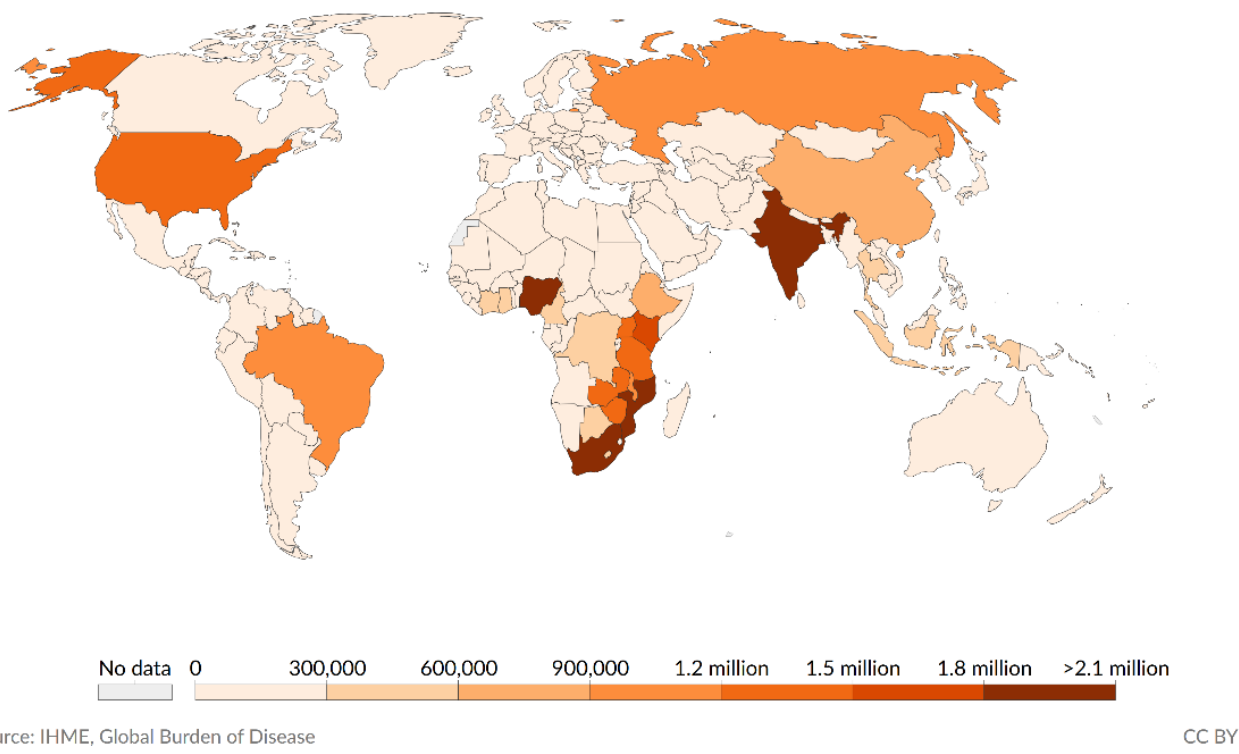

Figure 1.1 Map depicting the total number of people living with HIV-1 infection worldwide. The developing world is more highly affected by HIV-1, with higher rates of infection. Furthermore, women worldwide are disproportionally more susceptible to infection. Image taken from online site at OurWorldlnData.org ${ }^{8}$. 
The global challenge in curing HIV-1 is hindered by the sheer genetic diversity observed in the viral population. Despite the recent origin of the pandemic, the high mutation rate of HIV-1 has created an immense array of viral subtype. As of now, there are two types of HIV-1 known, the more virulent and widespread HIV-1, and HIV-2 which is localized in central Africa9 ${ }^{9}$. Because of the large genetic variability, HIV-1 is further divided into three groups: major (M), outlier $(\mathrm{O})$, and new $(\mathrm{N})^{10}$. The largest HIV-1 group, $\mathrm{M}$, can be further divided into subtypes, or viral clades. These clades, ranging from A to $\mathrm{K}$, are heavily localized in specific locations, and show high correlation with incidence rates and geography. In North America, clade B is the most common form of virus, while clade $\mathrm{C}$ is predominant in Africa ${ }^{11}$.

Despite the diversity of HIV-1 subtypes within the global population, all HIV1 particles share the same general structure. Each HIV-1 particle is comprised of two single stranded RNAs that are enclosed within a viral capsule comprised of p24 proteins ${ }^{12}$. In addition, the capsule is surrounded by an envelope (Env), comprised of glycoproteins 120 (gp120) and 41 (gp41). The viral envelope plays an essential role in enabling initial cell binding and virus entry. Within this viral capsule, reverse transcriptase and integrase proteins also exist, in addition to single-stranded RNAs ${ }^{13}$. These proteins are critical in "hijacking" the host cellular machinery for repurposing in viral replication.

As mentioned previously, HIV-1 glycoproteins gp120 and gp41 are essential for host cell binding and entry. Once inside the body through intercourse, mucus, or blood transmission, these glycoproteins allow HIV-1 to target CD4 T- 
helper cells, dendritic cells, macrophages, as well as Langerhans cells ${ }^{13}$. These cells express the cluster of differentiation 4 (CD4) receptor, as well as chemokine receptor 5 (CCR5), or chemokine receptor type 4 (CXCR4), which are the targets of viral glycoprotein interactions ${ }^{13}$. When the viral envelope glycoproteins bind to the CD4 receptor, a conformational change occurs on the viral envelope, causing additional binding to either CCR5 or CXCR4 co-receptors ${ }^{14}$. This binding leads to viral fusion with the cell surface, resulting in virus uptake. After entry, the viral proteins and RNA appropriate host cell machinery for the sole purpose of new viral particle production, although some infected cells can act as viral reservoirs, delaying the production of active virus.

Since the first confirmed isolation of HIV-1 in 1983, there has been a continued effort to eradicate HIV-1. The first antiviral agent to treat HIV-1 was Zidovudine (or azidothymidine, AZT), and was first approved by the Food and Drug Administration (FDA) in 1987. AZT is classified as a dideoxynucleoside reverse transcriptase inhibitor (NRTI), and inhibits the process of viral replication. However, due to high viral mutation rates and low tolerance in many patients, the sole use of AZT was not successful. Since the first introduction of AZT, dozens of new therapeutics have been introduced to combat the AIDS pandemic. Most of these therapeutics are used in combination to ensure complete viral inhibition. This course of action, called combinational highly active antiretroviral therapy (HAART) has shown success in treating infections ${ }^{14}$.

Currently, there are a variety of drugs that exist to treat HIV-1, with a total of six main types. These types: nucleoside/nucleotide reverse transcriptase 
inhibitors (NRTIs), non-nucleoside/nucleotide reverse transcriptase inhibitors (NNRTIs), protease inhibitors (PIs), fusion inhibitors, co-receptor inhibitors, and integrase inhibitors are categorized based on mechanism of action ${ }^{15}$. Nucleotide and nucleoside reverse transcriptase inhibitors (such as AZT) are agents that directly bind to the active site of the viral reverse transcriptase enzyme, which subsequently inhibits viral replication. In contrast, non-nucleotide/nucleoside reverse transcriptase inhibitors inhibit reverse transcriptase by binding to the active site of the protein. Protease inhibitors act to inhibit the formation of mature HIV-1 proteins, preventing new particle fabrication. These three classes act against HIV1 after the virus has entered the cell. In contrast, fusion inhibitors bind to and inactivate envelope (Env) proteins such as gp41, preventing HIV-1 entry into host cells. The use of co-receptor inhibitors can prevent gp120 and CCR5 interactions, again preventing viral entry into cells. Finally, integrase inhibitors, much like reverse transcriptase and protease inhibitors, work after viral infection and prevent the virus from integrating its genetic material into the host cell DNA.

This large array of HIV-1 therapeutics has been developed due to the highly mutagenic nature of HIV-1. Previous studies have shown administration of one agent will only inhibit infection temporarily, with viral mutations rendering the agent inactive ${ }^{16}$. Thus, HAART has been implemented, in which at least three drugs comprised of two different classes HIV-1 antiretrovirals are administered to treat HIV $-1^{16}$. These medications are taken orally daily, with several RTIs and at least one PI being used ${ }^{17}$. The use of drugs with different mechanism of actions reduces the risk of HIV-1 adaptation. Furthermore, another benefit of using a diversity of 
agents is the reduction of toxicity and off-target effects, relative to using the same class of agents ${ }^{15}$. With the implementation of HAART, patient life expectancy has increased tremendously by the continuous suppression of HIV-1, preventing AIDS.

In contrast to the more recent emergence of HIV-1, herpes simplex virus type-2 is estimated to have existed over 1 million years ago ${ }^{18}$. This virus is the root cause of genital herpes, of which there are two types. HSV-2 typically results in genital infection, while HSV-1 predominantly results in oral manifestations, and very rarely infecting the genital area. To date, there are over 530 million cases of HSV-2 infection, representing roughly $25 \%$ of the global adult population ${ }^{19}$. This high incidence of HSV-2 is largely attributed to the ability of virus to infect during asymptomatic periods, and to infect surface epithelial cells, as well latently infecting neuronal cells ${ }^{19,20}$. Although HSV-2 is not life threatening, no cure exists, resulting in the need for life-long maintenance.

Each HSV-2 virion is comprised of a viral capsid, or capsule containing double-stranded DNA ${ }^{21}$. The capsule itself is composed of proteins that form an icosahedral shape, surrounded by a lipid bilayer that forms the viral envelope. The lipid bilayer contains a variety of surface glycoproteins, five of which are essential for binding and entry to host cells: $g B, g C, g D, g H$, and $g L^{22,23}$. Unlike HIV-1, the capsid only encodes viral DNA; whereas the cellular machinery of the host provides the means for producing additional viral particles.

Initially, HSV-2 infects epithelial cells; however, following the initial infection the virus migrates to neuronal ganglia, providing a reservoir for latent infection. 
Cell entry is enabled by viral glycoproteins $\mathrm{gB}$ and $\mathrm{gC}$ located on the surface of the lipid bilayer. These two glycoproteins non-specifically bind to a cell surface proteoglycan called heparan sulfate ${ }^{20}$. The initial binding results in envelope conformational changes stabilized by both $\mathrm{gB}$ and the $\mathrm{gH}-\mathrm{gL}$ dimers, resulting in receptor-mediated cell binding and internalization via glycoprotein gD facilitation ${ }^{21}$. After this complex process, the virus enters the cell, resulting in the transcription of viral DNA via cellular machinery and subsequent viral replication.

Due to the latent, asymptomatic and irregular recurrences exhibited by the virus, no cure for HSV-2 exists. Additionally, relatively few agents are available to treat HSV-2, relative to HIV $-1^{24}$. The most commonly prescribed agents for HSV-2 treatment are: Acyclovir, Valaciclovir, and Famciclovir. Acyclovir is a guanosine derivative, which acts to specifically inhibit viral DNA polymerase activity after binding to the enzyme's active site. Additionally, Acyclovir was one of the earliest therapeutics available to combat HSV-2; however, due to the relatively low bioavailability of Acyclovir, newer therapeutics have been developed. One of the successors of Acyclovir is Valaciclovir, a prodrug that provides a 3-5-fold increase in bioavailability compared to Acyclovir. Last, Famciclovir, a prodrug of Penciclovir, possesses a similar mechanism of action as Acyclovir but can be topically administered $^{25}$.

Despite the numerous agents available to treat HIV-1 and HSV-2 infections, to date there are no FDA-approved agents that prevent or completely cure these infections. The lack of viable prevention agents for HSV-2 further contributes to the challenges of managing and combating HIV-1 infections. As previously mentioned, 
HSV-2 acquisition increases the risk of HIV-1 infection, by promoting inflammation, and more chronically causing small epithelial tears and genital lesions that increase the likelihood of HIV-1 transmission. Additionally, the asymptomatic nature of HSV-2 may result in the lack of awareness of infection, and hence increased risk of transmitting ${ }^{26}$. Therefore, the development of multipurpose regimens, active agents, or platforms that have the ability to mechanistically prevent both HSV-2 and HIV-1 infections are urgently needed.

Thus far, in terms of prevention, oral pre-exposure prophylaxis (PrEP) has been the primary method to prevent HIV-1 infection in high-risk individuals via daily oral administration. Truvada, a pill containing the antiretrovirals Tenofovir Disoproxil Fumarate (TDF) and Emtricitabine, is the only FDA-approved oral PrEP ${ }^{27}$. Clinical trials such as Centers for Disease Control and Prevention CDC 4243 and CDC 4940, and the International AIDS Vaccine Initiative IAVI E001 and IAVI E002 have demonstrated the efficacy of oral PrEP, with daily Truvada administration resulting in an HIV-1 prevention rate of $62 \%{ }^{28}$. Other clinical trials show similar results, with oral PrEP reducing HIV-1 acquisition in heterosexual couples $(75 \%)$, and in young adults $(44-62 \%)^{28}$.

Despite the success of oral PrEP, studies have shown that the protection imparted by oral PrEP is wholly dependent on strict user adherence. This was underscored in the VOICE (MTN-003) clinical trial, in which oral PrEP (and topical microbicide gels) failed to provide any meaningful protection due to a lack of user adherence ${ }^{29}$. Additionally, there are major concerns regarding long-term use and off-target side effects. Studies have shown that long-term administration of 
antiretrovirals may result in renal and bone toxicity due to the high doses required to overcome first-pass metabolism ${ }^{29}$. Furthermore, concerns exist over the use of TDF and Emtricitabine in both prevention and treatment regimens, with the risk of acquiring viral resistance to therapeutic agents, which would then render prophylactic methods, based on the same active agents, ineffective.

The challenges of oral PrEP have prompted the development of several topical delivery platforms that provide localized protection as well as the incorporation of biologics. Additionally, intravaginal delivery platforms are being developed to address the disparity of infections seen in women in the developing world by seeking to provide a convenient, low-cost, and discreet product for selfadministration. To date, intravaginal delivery has been an effective strategy to improve the localization of antiviral, antibacterial, antifungal, chemotherapeutic, and contraceptive agents within the female reproductive tract (FRT) ${ }^{30,31}$. One of the key advantages of topical PrEP, relative to oral is the avoidance of first-pass hepatic clearance and the harsh gastrointestinal environment, due to the dose localization. This results in an increase in drug bioavailability within target tissue and corresponding functional activity by decreasing off-target effects and systemic exposure $^{32}$. The inherent characteristics of the FRT, including its large surface area and low enzymatic activity, additionally make the FRT a favorable site for localized active agent administration and targeting ${ }^{33,34}$. Furthermore, localized administration provides the added benefit of decreasing potential drug toxicity. This results in more efficacious inhibition of HIV-1 at the site of transmission and initial infection $^{35}$. The potential of topical PrEP was first demonstrated in the CAPRISA- 
004 clinical study, where an intravaginally administered topical gel containing TFV was used to effectively reduce HIV-1 transmission by $39 \% 36,37$.

Although intravaginal delivery offers a variety of advantages to enhance the delivery of active agents ${ }^{38}$, challenges unique to the FRT must be overcome to provide efficacious prophylaxis and treatment. One of the most important components of the FRT is the mucus layer, which protects the epithelium and lamina propria from incoming pathogens (Figure 1.2). However, it can also act as a barrier, impeding active agent transport to underlying epithelial and immune cells ${ }^{39,40}$. In addition to these challenges, the frequent shedding and production of cervicovaginal mucus can decrease active agent retention, while bacterial flora, enzymes, and the acidic environment created by beneficial bacteria can contribute to metabolization and degradation of active agents, reducing efficaciousness. 


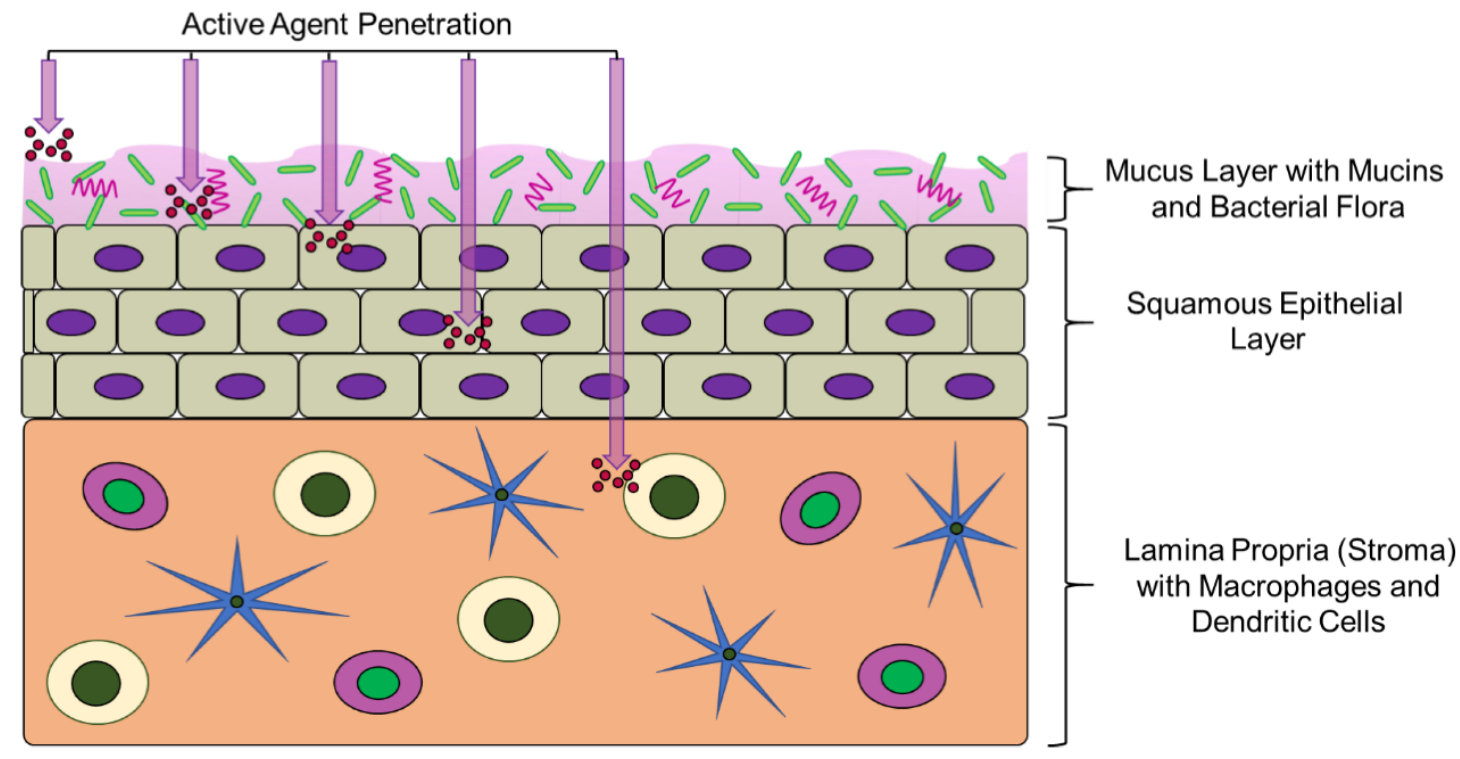

Figure 1.2 Schematic depicting the structure and specific layers of the vaginal mucosa that can act as a barrier to active agent transport (not to scale). The mucus layer of the female reproductive tract (FRT) frequently sheds and can immobilize active agents (shown in red), leading to decreased efficacy of the administered agents. The bacterial flora normally present within the FRT can also metabolize and degrade agents, further contributing to decreased efficacy. Last, the squamous epithelium can hinder transport to underlying immune cells present near the epithelial surface and/or in the lamina propria. 
To address these challenges, intravaginal delivery platforms have been formulated as solid or semi-solid dosage forms that include suppositories, tablets, capsules, gels, rings, and creams to enhance delivery to and retention in the $\mathrm{FRT}^{41-45}$. While these dosage forms have enabled high levels of active agent incorporation and localization, these traditionally used delivery platforms still face significant challenges, including difficulty of self-administration, economic feasibility, poor user-compliance, vaginal irritation, the need for frequent administration, and low residence times ${ }^{46}$. Of these platforms, intravaginal rings have provided the "gold standard" for long-term delivery due to their ability to sustain the release of one or multiple active agents for weeks to months, avoid leakage and loss of active agent, and improve drug stability ${ }^{47-51}$. However, some biological agents have difficulty withstanding the high temperature and solvent processes often required for fabrication, limiting their incorporation ${ }^{52}$.

Of these topical platforms, , fast dissolving inserts (FDIs), films, and tablets act to provide transient $(\leq 24 \mathrm{hr}$ ) protection, requiring use immediately prior to an exposure event, which may limit their utility ${ }^{35,53-55}$. Previous clinical trials assessing the efficacy of intravaginal gels demonstrated suboptimal results, which were attributed to a lack of user adherence ${ }^{53}$. Together, the current challenges of user adherence and the inability to provide sustained-release may limit the effectiveness of these delivery platforms.

In contrast to the previous technologies, IVRs, as mentioned earlier, provide the gold standard for sustained-release applications. Previous studies have shown that IVRs are able to release antiretrovirals for up to 6 months ${ }^{56,57}$. Additionally, 
IVRs have been developed to release hydrophilic agents, hormones, and recently biologics $^{58}$. The recent ASPIRE (MTN-020) clinical trial, has shown the promise of IVRs, with the reduction of HIV-1 infections by $27 \%$ in selected participants using IVRs encapsulating the antiretroviral dapivirine ${ }^{55}$. However, within this trial, it was seen that women ages 18-21, who are most vulnerable to infection, were less likely to adhere to an IVR regimen. Furthermore, in the recent HOPE study, despite an estimated user adherence of $90 \%$, intravaginal rings encapsulating dapivirine imparted only partial protection against HIV -1 , by reducing infections in $39 \%$ of women ${ }^{59}$. These challenges have prompted the development of new dosage platforms that may also integrate biologics to provide alternative options for women.

Concurrent with traditional antiretroviral development, the use of biologics in the field of STI prevention has increased within the last decade. Biologics, or active agents consisting of, or derived from living organisms, have shown the ability to provide enhanced specificity and decreased adverse off-target effects compared to traditional prophylactics and therapeutics ${ }^{60}$. However, many biologics lack oral bioavailability, preventing them from being used in oral treatment regimens. Given this, biologics may be incorporated into topical delivery platforms, which can overcome this limitation and additionally inhibit viral infection using different mechanisms of action, thus reducing the likelihood of acquiring antiviral resistance. One of the most promising biological microbicide candidates is the antiviral lectin, Griffithsin (GRFT). Originally derived from a species of red algae, GRFT has demonstrated potent antiviral activity against a variety of viruses, 
including HIV-1 and HSV-261-66. Among the biologically-based agents studied thus far, GRFT is one of the most potent against HIV-1, inhibiting activity with picomolar concentrations. GRFT acts against HIV-1 by specifically binding to viral gp120 glycoproteins, and inactivating the viral particle ${ }^{66,67}$. Additionally, previous studies using a $0.1 \% \mathrm{w} / \mathrm{w}$ GRFT gel protected mice from intravaginal infection with HSV$2^{65}$. This protection was attributed to the prevention of the cell-to-cell spread of HSV-2 and demonstrated the potential of GRFT in multipurpose (multi-virus) applications. Finally, GRFT has been shown to be highly stable and to have high resistance to denaturation, while simultaneously exhibiting biocompatibility and safety within human cells and murine models ${ }^{68,69}$. Despite the potential of GRFT, the lectin itself lacks oral bioavailability and is poorly absorbed, rendering oral administration of the antiviral agent unlikely, without the development of oralspecific formulations.

Although GRFT lacks oral bioavailability, it is an excellent candidate for delivery to the FRT. GRFT has demonstrated synergistic activity against viral infections, with antivirals such as of Tenofovir (TFV) and other biologics such as carrageenan $^{70-72}$. FDls encapsulating GRFT have demonstrated short-term protection against HSV-2 infection in vivo, with FDI-incorporated GRFT maintaining similar efficacy to free GRFT alone ${ }^{73}$. Additionally, GRFT stability and activity were demonstrated in gels, which are now under evaluation in clinical trials. However, despite the interest in GRFT, to date there is no delivery platform capable of prolonging the release of GRFT for more than $72 \mathrm{hr} 65,73,74$.

\section{Electrospun Fibers}


As a relatively new microbicide delivery technology, electrospun fibers (EFs) may provide a promising alternative for prolonged and localized agent delivery, with the potential to protect against multiple STIs. Some of the advantages of EFs include the ability to highly incorporate a diversity of active agents including drugs and biologics ${ }^{33,75}$, to tailor sustained-release by selecting different polymeric materials, and to maintain agent stability during the course of delivery ${ }^{76,77}$. Biodegradable polymers, such as poly(lactic-co-glycolic acid (PLGA) and poly(caprolactone) (PCL), are approved by the U.S. Food and Drug Administration (FDA) for therapeutic use, indicating their proven biocompatibility and potential for translation ${ }^{78}$. Together, these attributes have recently established polymeric EFs as an attractive platform for localized delivery against STIs.

Electrospun fibers have recently gained attention for intravaginal delivery due to their ease of use, ability to be fabricated into various geometries and sizes, and tunable release properties ${ }^{79,80}$. They have been considered for sustaineddelivery, a characteristic that is often desirable for intravaginal applications, due to their high surface area-to-volume ratio, degree of interconnected porosity, tunable pore sizes, surface-modification potential, interchangeable polymer options, and diverse fiber architectures that enable finer control over the rate, duration, and site of agent release ${ }^{81}$. Electrospun fibers have the additional advantage that they can be fabricated using a variety of natural or synthetic polymers to tailor release properties $^{82}$, and these polymer types are typically selected based on their biocompatibility, hydrophobicity, and related degradation properties. 
Over the past decade, researchers have begun to incorporate antiviral agents into polymeric EFs to prevent HIV-1 infection. One of the first studies to utilize EFs to combat HIV-1 developed pH-responsive fibers that encapsulated cellulose acetate phthalate (CAP) ${ }^{83}$. While CAP EFs exhibited long-term stability in low $\mathrm{pH}$ environments characteristic of the female reproductive tract, the EFs quickly degraded with the introduction of semen, to release active CAP and neutralize HIV-1 particles. Later research by the same group utilized surfacemodified polystyrene and polypropylene fibers to bind to and inhibit HIV-1 with higher efficacy than unmodified fibers alone ${ }^{84}$.

In addition to $\mathrm{pH}$-sensitive and surface-modified fibers, researchers have utilized EFs to provide tunable release of one or more incorporated active agents for HIV-1 prevention ${ }^{85,86}$. Polymer blends of polyethylene oxide (PEO) and poly(Llactic acid) (PLLA) were synthesized to encapsulate and tailor the release of the antivirals Maraviroc (entry inhibitor) and AZT for up to several weeks ${ }^{86}$. In another study, PLGA and PCL fibers were loaded with various concentrations of the antiretroviral TFV ${ }^{85}$. These fibers demonstrated sustained-release of TFV for 30 days, as well as efficacy against HIV-1 infection in vitro. Similarly, but less extensively for HSV-2, sustained-release delivery vehicles have been recently developed. In one study, ACV was incorporated into EFs. Release eluate collected up to 28 days post-release provided sustained protection against HSV-2 infection in vitro ${ }^{87}$.

One of the most significant factors that contributes to active agent release from fibers is the relative hydrophobicity of the selected polymer material ${ }^{88,89}$. In 
addition to polymer hydrophobicity, the medium (in vitro) or environment (in vivo) surrounding the fiber can impact drug release. Simulated vaginal and seminal fluids, often used to preliminarily assess intravaginal release, may alter the release of agents relative to testing in water or phosphate buffered saline (PBS) (in vitro) or in vivo, due to differences in viscosity, salt, and protein concentrations, as well as $\mathrm{pH}$. Therefore, depending on the degree of polymer hydrophobicity and the environment release it is tested in, the same encapsulated active agent can have distinctly different release profiles, in some cases ranging from hours to months ${ }^{90,91}$. Usually, independent of these conditions, the use of hydrophilic polymers often results in the immediate release of both hydrophilic and hydrophobic active agents due to the high solubility and degradation rate of hydrophilic polymers in aqueous environments ${ }^{92}$. Natural polymers such as collagen, gelatin, chitosan, elastin, and laminin, and synthetic polymers including poly(ethylene oxide) (PEO), polyvinyl alcohol (PVA), and polyvinylpyrrolidone (PVP) are examples of hydrophilic materials that have been fabricated into fibers with micron- and nanometer-scaled properties. In contrast, synthetic hydrophobic polymers including polycaprolactone (PCL), poly(lactic-co-glycolic acid) (PLGA), and polyurethane (PU) have demonstrated burst or sustained-release kinetics depending on the hydrophobicity of the incorporated active agent ${ }^{93-97}$. Moreover, synthetic hydrophobic polymers can also serve as a mechanical and structural basis for different fiber architectures in which the release of single or multiple encapsulants may be tailored by using more complex fiber designs or composites. Fiber release rates can also be optimized by adjusting the polymer molecular 
weight or hydrophilicity, for example, by adding hydrophilic groups such as aliphatic poly(phosphoester) to the polymer structure ${ }^{98}$. Together, these features have enabled the incorporation and release of a variety of antiviral, antimicrobial, and biological agents from fiber scaffolds ${ }^{99-101}$.

Active agent release from polymeric fibers typically occurs via diffusion, polymer degradation, and erosion ${ }^{89}$. When fibers are first administered, solvent or solution diffuses through the porous fiber matrix. Once in contact with the solvent or solution, the polymer matrix swells, loosening polymer chains and enabling the diffusion of active agents, dependent in part on molecular size. Concurrently, the fiber surface may undergo bulk erosion at a rate corresponding to polymer hydrophilicity. These features in combination with the large surface-to-volume ratio of the fibers allows for the increased diffusion of encapsulants relative to diffusion from non-porous bulk materials ${ }^{102}$. Traditionally, fibers have been electrospun as uniaxial fibers or fibers that comprise a single polymer or polymer blend and exhibit homogeneous morphology. Diffusion of active agents from more traditional uniaxial fibers is dependent upon the compatibility of the encapsulant, polymer, and surrounding eluant. In contrast with diffusion, polymer degradation is observed when fibers are exposed to aqueous environments, and polymer bonds are cleaved by either passive hydrolysis or enzymatic reaction ${ }^{103}$, resulting in slow degradation of the fiber scaffold. This degradation alters the distance between and size of interconnected pores, thereby impacting the diffusion and release of incorporated active agents. For most synthetic polymers, hydrolysis is the most common mechanism of degradation, although hydrolysis-resistant polymers have 
been utilized ${ }^{104}$, which significantly impact active agent release. As the fibers degrade, they can also undergo surface or bulk erosion, which is dependent upon solvent diffusivity into the fiber, polymer solubility, and overall fiber matrix dimensions ${ }^{105}$.

As a result of these mechanisms and the materials selected, electrospun fibers can tailor the release of encapsulated agents within different durations to achieve immediate (transient or rapid), short-term, or sustained-release. Within this review, we defined release as transient, when the complete release of active agents occurs within $24 \mathrm{~h}$ of administration; short-term, when the release occurs from one day to one week; or sustained, when the release of the active agent occurs over a duration of weeks to months. A schematic showing an example of these different potential release profiles is provided in Figure 1.3. Factors including the electrospinning parameters, polymer materials, fiber architecture, the resulting structure and morphology, and the distribution and amount of incorporated active agent each contribute to the resulting release kinetics and efficacy of delivery ${ }^{80}$. 

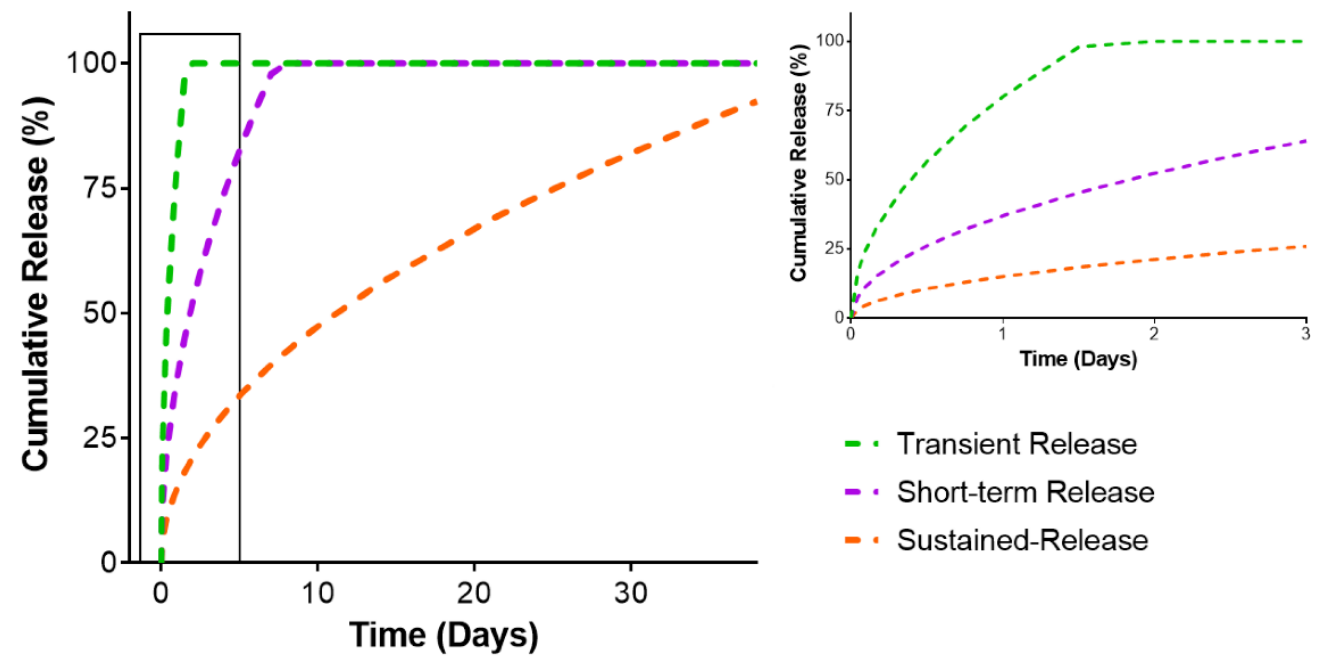

- Transient Release

- Short-term Release

- Sustained-Release

Figure 1.3 Schematic depicting examples of transient, short-term, and sustainedrelease profiles. 
Traditional uniaxial electrospun fibers in which each individual fiber is composed of a single cohesive polymer layer were the first fiber architectures to be fabricated ${ }^{106}$ and have been utilized in a variety of drug delivery applications over the past decade ${ }^{79,80,107-109}$. While uniaxial fibers offer high encapsulation efficiencies, cost-effectiveness, and ease of use, they have suffered from burst release and challenges in tailoring release properties ${ }^{80,90,110}$. These challenges are most evident in achieving the sustained-release of hydrophilic agents, often necessitating hydrophilic polymers to attain high encapsulation efficacy as well as hydrophobic polymers for sustained-release. More complex fiber architectures offer alternative options to address these limitations by combining different polymer types in distinct layers to modulate the release.

While the release characteristics of traditional uniaxial electrospun fibers have been thoroughly reviewed in literature ${ }^{76,111-115}$, to our knowledge, there has not yet been a review of the more advanced fiber architectures used to deliver active agents, nor a review that considers the impact these architectures may have on intravaginal delivery applications. Here, we seek to provide an overview of different polymer architectures including coaxial, multilayered, and nanoparticlefiber composites (Figure 1.4) as a function of the materials used to construct these architectures that have been utilized in a diversity of health applications. We seek to present different material combinations in these architectures to systematically relate material type and fiber architecture to active agent release kinetics. Last, we explore how lessons derived from these different architectures might be applied in the context of intravaginal delivery to address the needs of future topical sustained- 
release platforms for a given prophylactic or therapeutic application. The overall goal of this review is to provide a summary of different fiber architectures that have been useful for active agent delivery and to provide guidelines for the development of new formulations based on the knowledge obtained from previous work across other applications. While some of these more complex architectures have only recently been investigated relative to uniaxial fibers, they have demonstrated promise in enabling greater tunability of release and may be useful to apply as new dosage forms for intravaginal delivery and other similar applications. 
A

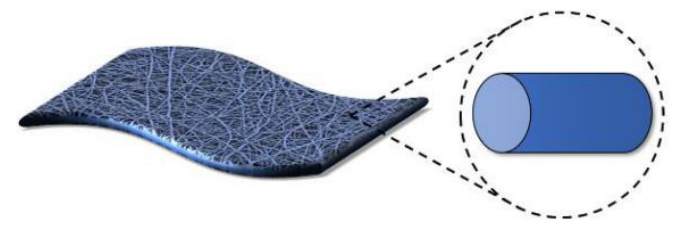

C

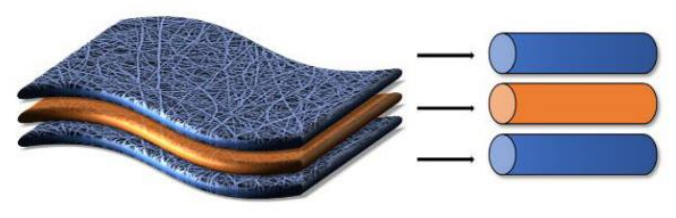

B

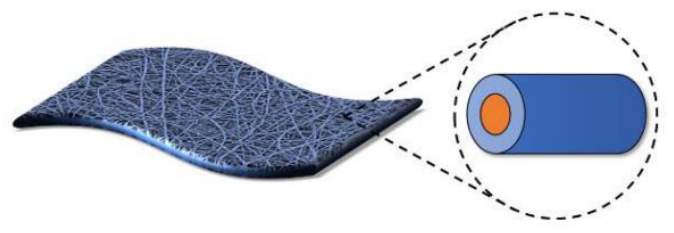

D

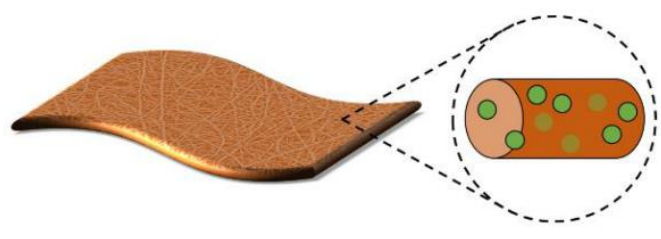

Figure 1.4 Schematic of different electrospun fiber composites. Diagrams representing (A) traditional uniaxial fibers, (B) coaxial fibers, (C) multilayered fibers, and (D) nanoparticle-fiber composites. (A) Uniaxial fibers are comprised of a single polymer or polymer blend (shown in blue) that is distributed homogenously throughout the fiber structure. (B) In contrast, coaxial fibers contain both core (orange) and shell (blue) layers that are chemically distinct. (C) Multilayered fibers result from sequentially electrospinning different fiber layers together or integrating individual layers post-fabrication. (D) Finally, nanoparticle-fiber composites consist of hydrophilic or hydrophobic fibers (orange) that encapsulate nanoparticles (green). 


\section{Coaxial Electrospun Fibers}

\section{Coaxial Architectures and Properties}

Coaxial electrospinning, adapted from uniaxial or single axial electrospinning, provides a multicomponent fiber scaffold that easily allows the tunable release of active agents ${ }^{116,117}$. Coaxial fibers are usually comprised of two parts, an outer protective layer or shell and an inner layer or core ${ }^{118}$, where encapsulants are typically localized (Figure 1.4B and Figure 1.5). Coaxial fibers can provide several advantages relative to uniaxially spun fibers. First, electrospinning the core and shell polymer solutions simultaneously through a coaxial spinneret allows for the design of unique fiber architectures. The thickness and ratios of the core and shell layers can be modulated, providing more reproducible fiber properties with a greater ability to alter encapsulant release relative to other fabrication methods. Additionally, coaxial electrospinning ensures that the active agent in the core phase is protected within harsh physiological environments, such as the female reproductive tract ${ }^{101}$. Furthermore, a variety of materials can be used as either the core or shell to finely regulate encapsulant release (Figure 1.5) ${ }^{116,119 .}$ 

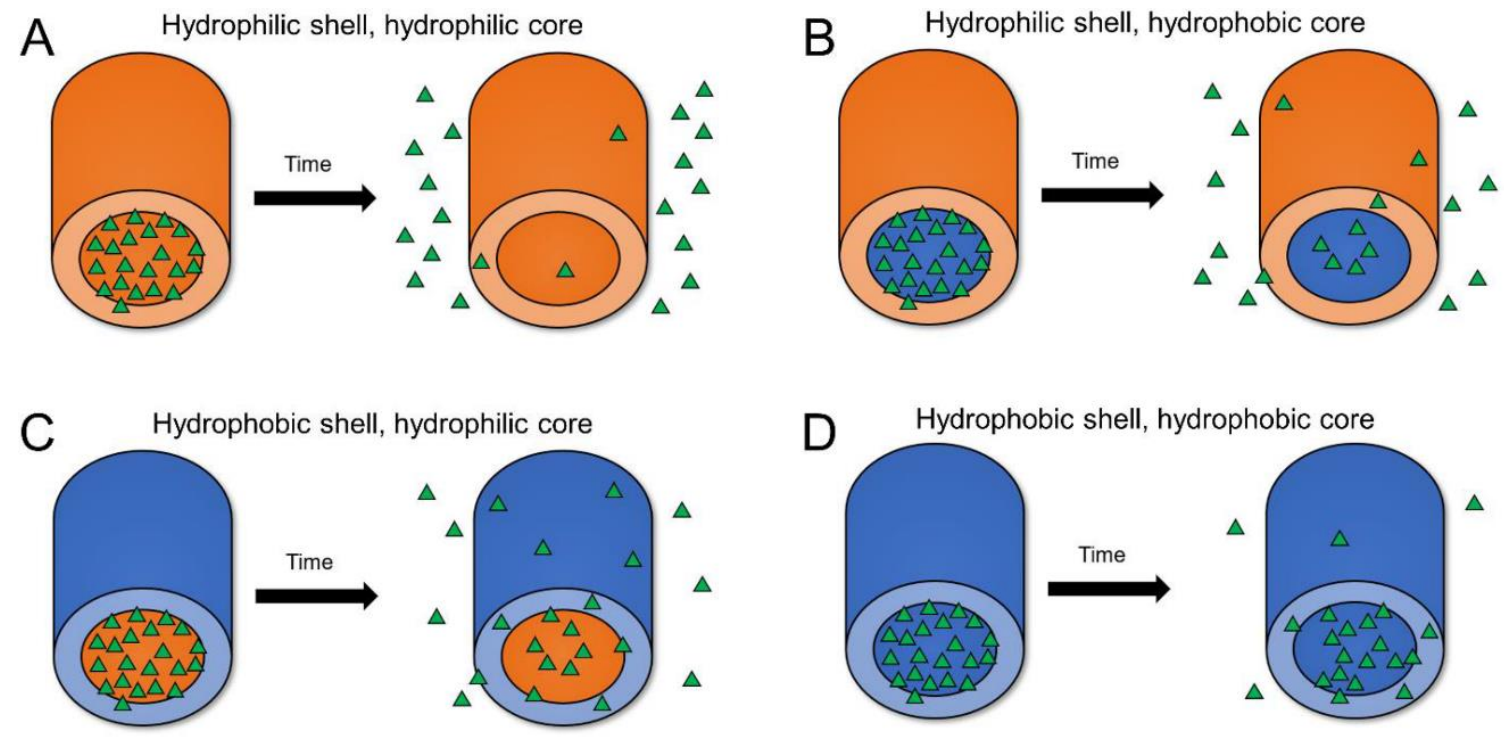

Figure 1.5 Schematic of anticipated release profiles from different coaxial fiber architectures. Generally, the release of encapsulants from coaxial fibers is dependent on the core and shell hydrophobicity. The release of active agents from coaxial fibers with (A) hydrophilic core and shell, (B) hydrophobic core and hydrophilic shell, (C) hydrophilic core and hydrophobic shell, and (D) hydrophobic core and shell are shown. Hydrophilic polymers (shown in orange) typically promote transient release, while more hydrophobic polymers (blue) are typically used to provide short-term or sustained-release. 
Despite these advantages, the added complexity of simultaneously electrospinning two or more polymer phases and the additional interactions between the core and shell solutions requires additional optimization relative to uniaxial electrospinning in terms of selecting compatible polymers and solvents. In addition to the core-shell architecture itself, the release profiles of active agents from coaxial fibers are impacted by solvent choice, polymer-solvent miscibility, the miscibility between core and shell solvents/solutions, solvent volatility, and layer thicknesses ${ }^{120,121}$. Solvent choice has been shown to alter fiber diameter and structure $^{122}$, thereby impacting active agent release ${ }^{123}$. Additionally, miscible core and shell solvents/solutions may lead to the partial dissolution of core encapsulants in the shell, whereas, immiscible core and shell solvents may promote material delamination at the core-shell interface, facilitating burst release of the core encapsulant. Therefore, the polymers and solvents for both core and shell layers must be selected based on their individual properties as well as their anticipated interactions ${ }^{124,125}$. In addition, solvent volatility and evaporation rate can affect the distribution and subsequent release of active agents, while the thickness of the polymer shell, polymer composition, and spinning conditions influence encapsulant diffusion rates ${ }^{126}$. Here, we discuss coaxial fibers as a function of their core-shell design, composition, and incorporated active agents to help relate these considerations to the resulting transient, short-term, or sustainedrelease characteristics.

\section{Release Kinetics from Coaxial Fibers}

\section{Transient Release (within $24 \mathrm{~h}$ )}




\section{Hydrophobic Shell—Hydrophilic Core}

Electrospun fibers can be designed to release the active agent immediately or within $24 \mathrm{~h}$ of administration if a rapid onset of action is needed for a given application ${ }^{127}$. Moreover, multiple active agents can be incorporated into different layers of a coaxial fiber (core or shell) to provide transient release.

For application to infectious diseases, coaxially spun fibers that demonstrate burst release followed by lower levels of short-term release may provide on-demand protection against incoming pathogens, increasing the immediate efficaciousness of agents by releasing initially high (burst) concentrations. This type of release can be achieved by employing coaxial fibers comprised of hydrophobic shells and hydrophilic cores. In one study, coaxial and triaxial fiber multi-drug delivery platforms that used PCL as the outermost shell released $\sim 15 \%$ and $\sim 80 \%$ of two different hydrophilic dyes, keyacid blue and keyacid uranine (KAB and KAU), from the PVP core and PCL shell fibers, respectively, within one hour ${ }^{116}$. In both the coaxial and triaxial fibers, the PVP core containing $\mathrm{KAB}$ was protected by the surrounding $\mathrm{PCL}$ layer containing KAU, which helped to extend the release of the remaining KAB to $24 \mathrm{~h}$. For the triaxial fibers, a blank PCL layer was electrospun between the outer PCL shell and the inner PEO core. In both the coaxial and triaxial fibers, KAU was released from the shell within $3 \mathrm{~h}$; however, the triaxial fibers better modulated the release of KAB from the core, releasing $50 \%$ less during the first hour. The burst release of the KAU dye, observed from both coaxial and triaxial fibers, was attributed to water penetrating the porous fiber shell, allowing transient release. In another example 
of coaxial fiber design, water-soluble PVP was used as a core with a hydrophobic ethyl cellulose (EC) shell to encapsulate hydrophobic compounds of either quercetin or ketoprofen. Using this architecture, $\sim 75 \%$ of both hydrophobic encapsulants were released within $24 \mathrm{~h}$.

\section{Hydrophilic Shell—Hydrophobic or Hydrophilic Cores}

Similarly, coaxial fibers that have hydrophilic shells can facilitate the rapid release of encapsulated agents with an initial burst release of 1 to $4 \mathrm{~h}$ followed by continued transient release within $24 \mathrm{~h}$ of administration. One architecture that has been adopted to achieve rapid- or on-demand release from coaxial fibers is a hydrophilic shell in combination with a hydrophilic or hydrophobic core. In one study, zein-PVP core-shell fibers were developed that incorporated the active agent in both the core (zein) and the shell (PVP) layers ${ }^{128}$. Zein, a natural, moderately hydrophobic polymer was used to achieve immediate and transient release of the hydrophobic drug, ketoprofen. A burst release of $43 \%$ was observed within the first hour, followed by transient release of the remaining ketoprofen over $10 \mathrm{~h}$. The initial burst release was correlated with rapid dissolution of the hydrophilic shell, while the more transient $10 \mathrm{~h}$ release was attributed to the hydrophobic core. In another study, the release profile of a hydrophobic drug, asiaticoside, was compared between coaxial fibers composed of chitosan cores with either a hydrophilic alginate and PVA-blended polymer shell or a hydrophobic centella triterpenes cream shell ${ }^{129}$. The coaxial fiber with the alginate-PVA shell demonstrated $80 \%$ more asiaticoside release relative to the centella control within

$10 \mathrm{~h}$, which was attributed to the shell hydrophilicity ${ }^{129}$. Additionally, the trend of 
burst release followed by more gradual transient release was attributed to rapid degradation of the alginate-PVA shell, followed by subsequent degradation of the chitosan core. While this example incorporated a polymer blend (alginate-PVA) as the hydrophilic shell, to be considered a core-shell structure, it should be noted that the material itself needs to be electrospinnable without other polymers. As this example demonstrates, hydrophilic polymers such as PVP, PVA, or PEO can be electrospun alone or in blends to create hydrophilic core and shell layers.

\section{Core-Shell Architectures with Similar Core-Shell Hydrophobicity}

Coaxial fibers comprised of both hydrophilic core and shell layers have also been investigated to provide transient release of active agents. For example, coaxial fibers fabricated with a hydrophilic PVP shell and hydrophilic cellulose acetate core were investigated. These coaxial fibers with both a hydrophilic core and shell released $31 \%$ of their hydrophobic encapsulant (epicatechin) within 10 min, followed by $80 \%$ release after $4 h^{130}$.

In addition to the utilization of materials with similar hydrophobicities, coaxial fibers consisting of identical core-shell materials have been fabricated to provide the rapid release of active agents. In one study, fibers with PVP shells and cores were investigated to provide rapid release of the hydrophobic drug, quercetin. The PVP shell-PVP core fibers released quercetin within one minute ${ }^{131}$, and this burst release was similarly observed in a separate study that used the same fiber formulation to deliver acyclovir ${ }^{132}$. In another study, the hydrophobic antibiotic, allyltriphenylphosphonium bromide, was incorporated within the core of coaxial 
fibers, and the volumetric ratios of core-shell solutions were varied to study release. Fibers comprised of zein-zein with core-shell volume ratios greater than $1: 2$ were found to suppress the burst release of the antibiotic, only releasing $15 \%$ within the first hour. In contrast, $35 \%$ and $45 \%$ of the antibiotic were released from fibers with a 1:1 core:shell volumetric ratio or blended fiber controls over the same duration ${ }^{133}$. In a separate study, a triaxial fiber in which all three layers were comprised of ethyl cellulose provided zero-order release of ketoprofen over $20 \mathrm{~h}$ due to the gradual increase in the drug content moving from shell to the core ${ }^{134}$. These studies highlight the role of the active agent distribution within the fiber layers, suggesting that encapsulant localization within the fiber core may enhance release.

Finally, the release of fluorescently labeled bovine serum albumin (BSA) from core-shell hydrogel nanofilaments composed of a poly(lactide-co- $\varepsilon$ caprolactone) (PLCL) shell and $\mathrm{N}, \mathrm{N}$-isopropylacrylamide (NIPAAm)/N,N'methylene bisacrylamide crosslinked core was studied. The crosslinker, N,N'methylene bisacrylamide, was used to polymerize NIPAAm during the electrospinning process. This study showed that by changing the NIPAAmcrosslinker (w/w) ratio from 4:1 to $37: 1$, the release of BSA increased from 0.15 to $0.7 \mathrm{ug} / \mathrm{mg}$ over $24 \mathrm{~h}$. However, in the absence of a hydrogel within the core, BSA showed nearly complete release over the same duration. This study demonstrated that the mechanical and corresponding drug release properties could be more finely tailored by altering the NIPAAm-crosslinker $(\mathrm{w} / \mathrm{w})$ ratio $^{135}$.

\section{Stimuli-Responsive Coaxial Architectures}


Another method to modulate the release of active agents from coaxial fibers is to integrate stimuli-responsive layers to precisely release agents in response to surrounding physiological conditions ${ }^{136}$. Unlike stimuli-responsive uniaxial fibers, the more complex interactions between the core and shell layers in coaxial fibers can provide increased control of active agent release via $\mathrm{pH}$ - or other stimuli-based mechanisms. A variety of natural and synthetic materials have been investigated for their use in $\mathrm{pH}$-responsive applications. In one example, a coaxial fiber comprised of a lecithin-diclofenac sodium core and a Eudragit S100 shell provided the $\mathrm{pH}$-responsive release of ferulic acid for $10 \mathrm{~h}^{137}$. Ferulic acid release was facilitated under conditions of neutral $\mathrm{pH}(\mathrm{pH} 7)$, with minimal release occurring in a more acidic $(\mathrm{pH} 2)$ environment. Another $\mathrm{pH}$-sensitive polymethacrylate-based copolymer ${ }^{137-139}$, Eudragit EPO, was used to fabricate $\mathrm{pH}$-responsive antibacterial fibers. Here, Eudragit EPO cores, which dissolve below $\mathrm{pH} \mathrm{5,} \mathrm{were} \mathrm{used} \mathrm{in}$ combination with Eudragit L100 shells, which dissolve at a pH greater than 6 . These coaxial fibers provided $\mathrm{pH}$-responsive release for an hour under slightly acidic conditions $(\mathrm{pH} \mathrm{6)}$ while demonstrating attenuated release in very acidic conditions $(\mathrm{pH} 2)^{29}$. Additionally, two separate studies investigated coaxial fibers comprised of Eudragit S100 shells and PEO cores to stimulate $\mathrm{pH}$-responsive release within the gastrointestinal tract ${ }^{140,141}$. In both studies, the release of hydrophobic indomethacin and hydrophilic mebeverine hydrochloride agents was minimal ( 10\%) after $2 \mathrm{~h}$ under acidic conditions, followed by rapid release for $6 \mathrm{~h}$ when switched to neutral conditions ( $\mathrm{pH}$ 7.4). Coaxial fibers comprised of cellulose acetate phthalate shells with polyurethane cores, as well as gelatin-sodium 
bicarbonate shells with PLCL cores have also been used to provide similarly rapid $\mathrm{pH}$-responsive release of ciprofloxacin and rhodamine B (Rhd B). These studies demonstrated the potential of coaxial fibers as $\mathrm{pH}$-sensitive delivery systems ${ }^{142,143}$.

Coaxial fibers with other stimuli-responsive properties have been investigated for on-demand, rapid release applications. Although studies with other stimuli-responsive systems have been limited, one study investigated the use of self-immolative polymers, or polymers that depolymerize when exposed to specific external stimuli, for rapid stimuli-responsive release ${ }^{144}$. In this study, selfimmolative fibers comprised of dibutyltin dilaurate and phenyl (4(hydroxymethyl)phenyl) carbamate were blended with polyacrylonitrile and used as shells to surround PVP cores. The fibers provided minimal release of KAB dye when incubated in water; however, the fibers depolymerized when exposed to trifluoroacetic acid, resulting in zero-order release of $\sim 40 \%$ dye within a week.

\section{Short-Term Release (One Day to One Week)}

\section{Hydrophobic Shell—Hydrophilic Core}

A key advantage of short-term release specifically for intravaginal delivery is that the burden of frequent or daily administration may decrease, thereby increasing user adherence of prophylactics and therapeutics. Traditionally, hydrophobic materials have been well-suited to provide longer durations of release (depending on the encapsulant) due to their decreased degradation rates in aqueous environments. For more traditional uniaxial hydrophobic fiber platforms, most hydrophobic small molecule drugs or larger macromolecules achieve release 
for up to one week due to the similar hydrophobic properties of the polymer and encapsulant ${ }^{38}$. This compatibility allows for hydrophobic encapsulants to partition more evenly within and distribute throughout hydrophobic polymers. However, hydrophilic agents, which have low solubility in nonpolar polymers, often partition to the fiber surface, resulting in burst release and suboptimal short-term and/or sustained-release properties. To address this challenge, coaxial fibers in which hydrophilic agents are encapsulated within a hydrophilic core and surrounded by a protective hydrophobic shell can prolong and adjust the release of hydrophilic molecules.

The use of coaxial fibers with hydrophobic shells and hydrophilic cores has been shown to extend the release of many encapsulants ${ }^{118,145,146}$. In one study, a coaxial fiber comprised of a hydrophobic ethyl cellulose shell with a hydrophilic PVP core was investigated for short-term release. These fibers released maraviroc over a duration of hours to days depending on the thickness of the hydrophobic shell, which was modulated via flow rate and total electrospun volume. The increased thickness of the hydrophobic shell extended encapsulant release from $24 \mathrm{~h}$ to five days by increasing the shell-to-core volume ratio from 0.5 to $4^{145}$. In another study, a PCL fiber shell surrounding a PVP-graphene oxide blended core was studied. These fibers released $65 \%$ of hydrophilic vancomycin hydrochloride within $4 \mathrm{~h}$ and attained full release of vancomycin after $96 \mathrm{~h}^{147}$. Although this coaxial fiber provided short-term release, the long-term safety of graphene oxide within the FRT is unknown, and further studies are required to assess its safety in intravaginal delivery applications. Finally, a coaxial fiber composed of a synthetic 
hydrophilic poly-cyclodextrin core and hydrophobic poly(methacrylic acid) shell reduced the burst release of a hydrophilic drug, propranolol hydrochloride, by $50 \%$, and extended release to $180 \mathrm{~h}$ relative to the 140 hour release obtained from uniaxial fibers ${ }^{148}$.

\section{Hydrophobic Shell-Hydrophobic Core}

In addition to the widely used hydrophobic shell-hydrophilic core coaxial architectures, the use of hydrophobic materials in both the core and the shell layers has also been investigated to provide the short-term release of active agents. In one study, a PCL core surrounded by an outer PCL shell was used to prolong the release of the antibiotic ampicillin. Ampicillin, a hydrophilic compound, normally localizes to the surface of PCL when spun as a uniaxial fiber, resulting in burst release $^{149}$. As an alternative, a $4 \%(\mathrm{w} / \mathrm{v})$ PCL solution was used to fabricate an ultra-thin shell to delay release. In addition, the parameters for coaxial electrospinning were modified using dilute sheath solutions to improve the control of fiber diameter and morphology. The resulting coaxial fiber efficiently encapsulated ampicillin and provided short-term release for $\sim 80 \mathrm{~h}^{149}$. In another study, coaxial fibers comprised of a zein shell with a PCL core reduced the burst release of the hydrophilic antibiotic, metronidazole, achieving short-term release for more than four days ${ }^{125}$.

\section{Stimuli-Responsive Coaxial Architectures}

Coaxial fibers exhibiting stimuli-responsive properties have also been investigated to provide short-term release of active agents. As one example, 
poly $(\mathrm{N}$-isopropylacrylamide), a thermoresponsive polymer, was used as a core layer in combination with an ethyl cellulose and anhydrous ethanol shell solution. At room temperature, poly( $\mathrm{N}$-isopropylacrylamide) exhibits hydrophilic properties; however, at temperatures above $32{ }^{\circ} \mathrm{C}$, the polymer demonstrates more hydrophobic characteristics. At room temperature and after $55 \mathrm{~h}$, the fibers released $65 \%$ of ketoprofen in PBS, while only $40 \%$ of the same drug was released at $37^{\circ} \mathrm{C}^{150}$.

\section{Blended Polymers in Coaxial Architectures}

Another method of prolonging release is to use blended polymers to formulate coaxial fibers, which can decrease fiber wettability. One study combined gelatin, a natural hydrophilic protein, with the hydrophobic polymer, PCL, to create coaxial fibers with increased hydrophobicity and mechanical stability relative to gelatin alone ${ }^{151}$. In one study, the release of hydrophilic doxycycline was measured from three different fiber architectures-a uniaxial PCL-gelatin blended fiber, coaxial fibers with three different cores (PCL, gelatin, or a PCL-gelatin blend) and a PCL-gelatin blended shell, and a triaxial fiber with both a PCL-gelatin blended core and outer shell and an intermediate gelatin layer. Among these five designs, uniaxial PCL-gelatin blended fibers released the most doxycycline within $24 \mathrm{~h}$ (90\%), while coaxial fibers with a PCL-gelatin core and shell released the least (50\%). Additionally, only coaxial fibers with either a PCL-gelatin or gelatin core prolonged release over five days. Furthermore, the other architectures including the uniaxial PCL-gelatin blend, coaxial fiber with PCL core, and triaxial fibers failed to release doxycycline for more than $30 \mathrm{~h}$. The burst release observed in fibers 
with PCL cores was attributed to the lack of compatibility between the hydrophobic PCL cores and hydrophilic encapsulant, which caused doxycycline to localize on the core surface. Additionally, the subsequent suboptimal encapsulant release was attributed to low water penetration into the hydrophobic core. These studies demonstrate that utilization of both hydrophobic and hydrophilic polymers alone or as blends can modulate the short-term release of hydrophilic encapsulants due to the variation in the permeability of different layers and core-encapsulant interactions.

\section{Sustained-Release (One Week to Multiple Months)}

\section{Hydrophobic Shell—Hydrophilic Core}

Similar to fibers that provide short-term release, fibers designed for sustained-release commonly use hydrophobic polymers as the outer shell to prevent the fiber from undergoing rapid hydrolysis. Studies have demonstrated that the most promising coaxial architecture to achieve sustained-delivery utilizes a hydrophobic shell and hydrophilic core ${ }^{38}$. A polymer that is frequently used in coaxial fibers to provide sustained-release is poly(lactic-co-glycolic acid) (PLGA). In one study, a coaxial fiber composed of a PLGA shell was used to shield a hydrophilic core consisting of tragacanth gum. The encapsulant, tetracycline hydrochloride, served as a model hydrophilic agent. Investigators observed that PLGA (shell)-tragacanth gum (core) coaxial fibers diminished burst release and provided sustained-release of tetracycline hydrochloride for 75 days, releasing $68 \%$ of tetracycline hydrochloride during this period ${ }^{152}$. In another study, a PLGA 
(shell)-polyethylenimine (PEI, core) architecture was used to prolong the release and stability of bone morphogenetic protein-2 plasmid (pBMP2-2). The hydrophilic PEI core was used to encapsulate and retain the bioactivity of pBMP2-2, while the hydrophobic PLGA shell was used as a protective barrier to prolong release. When compared to uniaxial PLGA-PEI blended fibers, the PLGA (shell)-PEI (core) coaxial fiber exhibited both improved bioactivity and prolonged release of the pBMP2-2 plasmid. The coaxial fiber released $80 \%$ of the plasmid over 20 days, while the uniaxial fibers released the same amount over seven days ${ }^{153}$.

Polymers other than PLGA have been used as hydrophobic shells to sustain the release of active agents from coaxial fibers. One study formulated coaxial fibers containing a hydrophilic dextran core and hydrophobic PCL shell. The addition of polyethylene glycol (PEG) to the PCL shell increased the release of the encapsulated BSA by forming pores in the shell layer. Although all fibers released $\sim 20 \%$ BSA within the first $24 \mathrm{~h}$, increasing the PEG concentration increased the amount of BSA released over extended durations. Interestingly, all fibers demonstrated sustained-release regardless of PEG concentration; coaxial fibers fabricated with $5 \%$ PEG shells released $\sim 60 \%$ BSA, while fibers containing $40 \%$ PEG shells released $90 \%$ BSA over 27 days ${ }^{154}$. In another study, the relationship between PEG (core):PCL (shell) molar ratio and the release of BSA or lysozyme was investigated. The thinnest shell layers with a core:shell molar ratio of 1.59 and a core flow rate of $2 \mathrm{~mL} / \mathrm{h}$ provided complete release of both encapsulants within 24 days, compared to only $50 \%$ release from thicker fibers with a core:shell molar ratio of 0.32 and a core flow rate of $0.6 \mathrm{~mL} / \mathrm{h}$. Moreover, the fibers preserved the 
bioactivity of lysozyme and released BSA over 29 days, with no noticeable differences between BSA and lysozyme release rates $^{155}$. In addition to conventional coaxial spinning, the use of emulsion electrospinning has also been investigated to fabricate coaxial fibers, which can be electrospun using a uniaxial spinneret ${ }^{117}$. One study that used emulsion electrospinning fabricated core-shell fibers composed of a PEG-poly(d,I-lactic acid) shell and methyl cellulose core to minimize the burst release of lysozyme ${ }^{156}$. The release of lysozyme from the core was achieved over 15 days and was dependent on the percent of lysozyme loaded, while the structural integrity and bioactivity of lysozyme was protected by the shell. A later study compared these same coaxial fibers to blended uniaxial fibers composed of PCL and PEG and showed that the coaxial fibers improved sustained-release by releasing $\sim 50 \%$ of BSA over 35 days relative to blended fibers, which released $\sim 75 \% \mathrm{BSA}^{157}$.

Another study explored the effects of multiple processing parameters, including PEG and PCL concentrations, PEG molecular weight, encapsulant concentration, and fiber diameter, in modulating the release of plasmid DNA (pDNA). Plasmid DNA was encapsulated in a PEI core, and a non-viral gene delivery vector ( $r$-PEI-HA) was incorporated within a PCL shell ${ }^{158}$. An increase in fiber diameter was observed with an increase in all of the three other parameters, while the loading and release of r-PEI-HA were correlated to pDNA concentration in the fiber core and PEG molecular weight. The fibers formulated with high PEG molecular weight and low pDNA concentration exhibited $\sim 30 \%$ release of $r-\mathrm{PEI}-$ 
HA over 60 days, while the fibers with high pDNA concentration and low molecular weight PEG completely released pDNA within 60 days.

\section{Core-Shell Architectures with the Same Core-Shell Hydrophobicity}

Although coaxial architectures with similar core and shell hydrophobicities have been utilized to obtain transient and short-term release, coaxial fibers that use the same materials have been less frequently investigated to provide sustained-release. In one study, PLGA was utilized in both the core and shell layers to investigate the effect on vancomycin and ceftazidime delivery ${ }^{159}$. Both hydrophilic drugs were encapsulated within the core PLGA layer and exhibited similar burst release kinetics within the first day, followed by a second phase of more gradual release over five to ten days. Ninety percent of the antibiotics were released after 11 days, followed by complete release after 25 days, with the more gradual release attributed to the PLGA barrier layer.

\section{Applications for Intravaginal Delivery}

The enhanced tunability and versatility provided by the core and shell layers of coaxial fibers make them excellent candidates for intravaginal delivery applications. While uniaxial fibers have been studied for sustained- and stimuli-

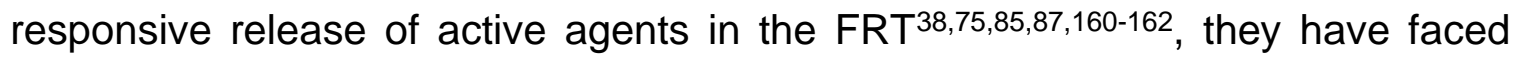
challenges in providing the sustained-release of therapeutically relevant concentrations of individual active agents and effectively modulating the release of multiple agents core ${ }^{38}$. Often, compatibility between the polymer and encapsulant can pose challenges to achieving sustained-release with uniaxial 
fibers, while coaxial fibers may circumvent this issue by integrating two different polymers, enabling the separation of agents within a compatible polymer formulation (core or shell). Moreover, the additional outer shell can help to modulate release. One can envision that with a coaxial architecture, multiple agents may be delivered against a particular infection to provide a synergistic effect or to provide protection against multiple types of viral or bacterial infections. Together, these features allow for enhanced tunability with the option of providing immediate to short-term release for on-demand applications while also providing long-term release that may be particularly useful in prophylactic or contraceptive applications.

A variety of release kinetics can be attained from coaxial fibers by using different combinations of materials in the core and shell layers. Transient or rapid release of active agents is often accomplished with the use of hydrophilic polymers due to their rapid dissolution in aqueous environments. To achieve short-term release extending to one week, a hydrophilic core in combination with a hydrophobic shell is the most frequently used architecture, enabling the slow dissolution of the shell layer, which acts as a barrier to encapsulant diffusion from the core. For sustained-release applications that require delivery on the order of weeks to months, hydrophobic polymers such as PLGA and PCL are often selected as shell polymers due to their slower degradation kinetics and biocompatibility. Yet, due to the number of parameters involved in the synthesis of coaxial fibers, two similar architectures may still be tailored to perform very differently by altering physical versus chemical properties. An example may be 
seen in which fibers composed of similar or even the same polymers display very different release rates due to the modulation of shell thickness. In these cases, thinner shells have been shown to provide more transient release, while increasing the shell thickness delays or alters the trend to more gradual release.

Coaxial fibers have been investigated previously for intravaginal delivery $^{142,145}$. In one study, maraviroc release from coaxial fibers was adjusted by varying the drug loading and solution flow rates to provide release over five days $^{145}$. In addition, $\mathrm{pH}$-responsive coaxial fibers have been fabricated to react in the presence of semen by utilizing the $\mathrm{pH}$-sensitive polymer cellulose acetate phthalate as a shell. The outer shell dissolved immediately after exposure to PBS, promoting $\mathrm{pH}$-responsive release of $\mathrm{Rhd}^{142}$.

Although coaxial fibers have shown promise in general drug and initial intravaginal delivery applications, further refinements are required to expand their overall utility. First, compatibility between the solvents of the two polymer electrospinning solutions may limit the potential combinations of core-shell materials and encapsulated agents to achieve successful electrospinning. Additionally, residual solvents from the electrospinning process may interact with and inactivate encapsulated active agents in the core layer. Therefore, while research in coaxial fiber design is still ongoing, other fiber architectures such as multilayered fibers may offer additional advantages to advance intravaginal delivery.

\section{Multilayered Electrospun Fibers}




\section{Multilayered Fiber Architectures and Properties}

Multilayered fibers can provide layer-by-layer delivery platforms that are relatively simple and inexpensive to fabricate while allowing for the encapsulation of different active agents within the individual layers. The topology, thickness, and composition of each individual layer can be easily tuned to provide different release properties based on the envisioned application. Moreover, multilayered fibers have been shown to have increased mechanical stability and flexibility compared to coaxial fibers ${ }^{163}$. While the interactions between two or more polymer solution interfaces must be considered for coaxial fibers, multilayered fibers can be fabricated from normally incompatible polymers due to their sequential versus simultaneous fabrication process.

Electrospun multilayered fibers can be fabricated by sequential layering, stacking, or interweaving fibers ${ }^{164-166}$. In sequential layering, the first layer of polymer is electrospun onto a collector, followed by electrospinning additional polymer layers directly onto the same collector. In comparison, "stacking" fibers refers to individually electrospinning each layer separately and subsequently adhering individual layers together post-spin. Stacked fibers share similar physical properties with sequentially-layered fibers, enabling temporally-programmed or spatially-specific delivery of active agents ${ }^{167}$. Finally, the fabrication of interwoven fibers utilizes dual or multiple-syringes to simultaneously electrospin two or more different polymer solutions (usually one hydrophilic and hydrophobic) onto the same collector. In contrast to fibers produced using the sequential layering and stacking processes, which have distinct, separate layers of polymeric fibers, 
interwoven fibers result from the blending of these different polymer solutions from syringes placed opposite of or adjacent to each other into one integrated layer ${ }^{168-}$ 170. This technique seamlessly integrates both hydrophilic and hydrophobic polymers in a way that prevents unwanted interactions between the electrospun polymer solutions ${ }^{170,171}$ while enabling the porosity of the hydrophilic fibers to be altered to more finely tune fiber degradation ${ }^{172}$. Although interwoven fibers do not have a shell layer, the interwoven architecture has been beneficial in promoting cell adhesion and growth and has the potential to more finely modulate active agent release via porosity-based mechanisms for drug delivery applications ${ }^{173,174}$.

Regardless of fabrication technique, multilayered fibers are beneficial in that they can temporally modulate the release of multiple agents from a single delivery platform and can provide additional tunability by modulating the barrier or discrete layers of the multilayered structure (Figure 1.6). In addition, the ability to impart spatially-specific release-where specific layers of the multilayered fiber possess distinct release profiles-is a key advantage of this architecture. This advantage may be envisioned for intravaginal delivery applications where one layer provides rapid active agent release to the mucus while another layer enables sustaineddelivery specific to underlying epithelial or immune cells ${ }^{164,165}$. For interwoven multilayered fibers, studies have shown that the incorporation of a hydrophilic polymer can alter the overall porosity and wettability ${ }^{172,175,176}$, while using a hydrophobic outer layer in multilayered fibers (similar to coaxial fibers) can decrease surface wettability and corresponding active agent release ${ }^{177}$. 
A

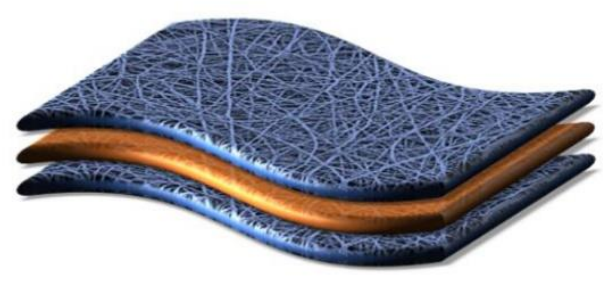

B

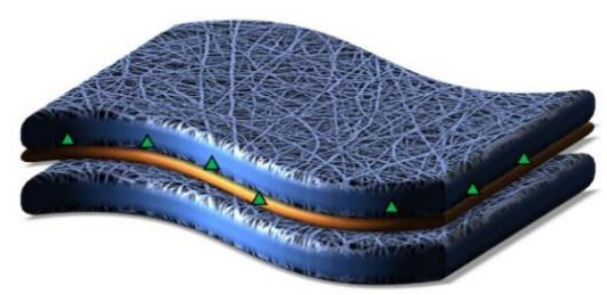

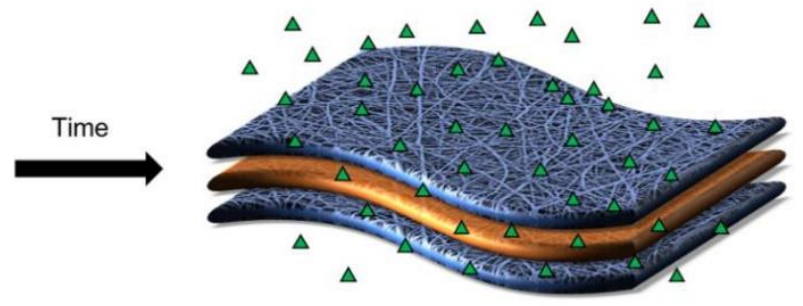

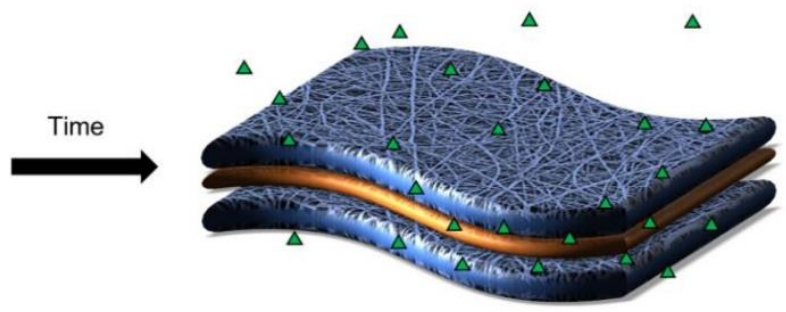

Figure 1.6 Schematic of anticipated active agent release from multilayered fibers. One method to modulate the release of active agents (shown in green) is to vary the thickness of the outer layer (shown in blue). (A) A thin outer layer provides both rapid burst release and limited sustained-release of encapsulants. (B) In contrast, increased outer layer thickness can delay the release of some active agents. 
While the process of creating multilayered fibers is well established, more work is required to elucidate how each polymer layer impacts release kinetics. Physical properties including the pore size, fiber diameter, and thickness of traditional uniaxial fibers are known to impact the delivery kinetics of active agents from individual layers. Thus, the presence of one or more fiber layers can contribute to the complexity in establishing and predicting the release kinetics of diverse active agents from differently layered architectures. Despite these considerations and complexities, the adoption of different layering techniques to create multilayered fibers can achieve diverse patterns of release for transient, short-term, and sustained-release applications.

\section{Release Kinetics from Multilayered Fibers}

\section{Transient and Short-Term Release}

Multilayered fibers have shown promise in providing transient and shortterm release of active agents. Conventionally, a hydrophilic layer serves as a reservoir for active agents, while hydrophobic materials provide an outer shell layer to prolong release. One study utilized a multilayered fabrication approach to encapsulate the hydrophobic antibiotic, gentamicin, in a hydrophilic PVA center layer and utilized a PU outer layer to envelop the inner PVA fiber ${ }^{178}$. Three separate fibers were fabricated by altering the thickness of the PU outer layer

between 3.4 and $8.1 \mu \mathrm{m}$. The release of gentamicin was modulated with the thinnest PU layer $(3.4 \mu \mathrm{m})$ demonstrating complete release within $1 \mathrm{~h}$, relative to $10 \%$ release obtained from the thickest layer $(8.1 \mu \mathrm{m})$. Furthermore, the thickest 
PU layer continued to release gentamicin for $24 \mathrm{~h}$. Another study using interwoven electrospun fibers containing PEO and PCL demonstrated that by adjusting the ratio of the two polymers, tunable fiber degradation could be achieved from the resulting changes in pore size and porosity ${ }^{170}$. Although this study investigated interwoven fibers to enhance cell infiltration through the pores, the use of sacrificial fiber layers may be applied to modulate active agent release from the fibers for intravaginal delivery applications ${ }^{170}$.

In addition to modulating the outer layer thickness and overall fiber composition, alterations to the number of layers have been shown to impact active agent release. In one study, fibroin-gelatin blended uniaxial fibers exhibited release of trypan blue, fluorescein isothiocyanate (FITC)-inulin, and FITC-BSA within minutes ${ }^{179}$. In contrast, multilayered fibers composed of the same materials extended the release of all three model compounds to 28 days ${ }^{179}$. In another study, dual-release, multilayered electrospun fibers containing the model dyes, 5,10,15,20-tetraphenyl-21H,23H-porphinetetrasulfonic acid disulfuric acid (TPPS) and chromazurol B, were encapsulated in four-layered PLCL $(75: 25)$ fibers. The release rate and duration of the dyes were controlled by the fiber diameter and individual fiber layer thicknesses. Minimal release of both dyes was observed for the first $15 \mathrm{~min}$, followed by a quasi-linear release profile for up to $4 \mathrm{~h}$. However, increasing the thickness of dye-loaded layers resulted in higher quasi-linear release rates due to the reduced density of the fiber surface ${ }^{180}$. In another study, the transient release of ketoprofen was achieved using trilayer fibers composed of two EC outer layers surrounding a center PVP fiber. These fibers provided nearly 
complete release of ketoprofen within $24 \mathrm{~h}^{164}$. Last, asymmetric multilayered polylactide fibers with different designs on each side were fabricated to prevent liver cancer recurrence by promoting one-sided prolonged chemotherapeutic release ${ }^{181}$. The fiber was composed of five poly(lactic acid) (PLA) layers, with each layer serving as either a barrier to release or a drug encapsulating reservoir. In vivo studies in a murine model demonstrated tumor suppression for at least four days, indicating that the multilayered fiber may provide localized chemotherapy for short-term durations ${ }^{181}$.

Multilayered fibers with stimuli-responsive properties have also been investigated for transient and short-term release applications. In one of the first studies to investigate multilayered architectures, the $\mathrm{pH}$-responsive polymers, poly(acrylic acid) (PAA) and poly(allylamine hydrochloride) (PAH), were electrospun together to create a blended fiber. These fibers were loaded with a low molecular weight cationic molecule, methylene blue, and demonstrated rapid release of methylene blue ( 10 $\mathrm{min}$ ) at a neutral $\mathrm{pH}(7.4)$. However, by gradually adjusting the $\mathrm{pH}$ from 6 to 2 in aqueous solutions, the step-wise $\mathrm{pH}$-responsive release of methylene blue was achieved over three and a half days. Building upon this work, the effect of coating the fibers with a thermoresponsive polymer blend, poly(N-isopropylacrylamide)-PAA, or perfluorosilane was assessed. The addition of the thermoresponsive poly( $\mathrm{N}$-isopropylacrylamide)-PAA coating modulated methylene blue release via temperature. Above a critical temperature, the thermoresponsive polymer became insoluble and formed intramolecular hydrogen bonds, which led to the release of methylene blue within 50 min (PBS, pH 7.4). In 
comparison, coating with perfluorosilane modulated release for up to $20 \mathrm{~h}$ at neutral $\mathrm{pH}$. When both the $\mathrm{pH}$-responsive and multiple layers of thermoresponsive polymers were integrated and evaluated at 25 and $40{ }^{\circ} \mathrm{C}$, dye released for a maximum of $10 \mathrm{~h}$ regardless of layer thickness ${ }^{182}$.

\section{Sustained-Release}

The ability of multilayered fibers to provide long-term release has been demonstrated in a variety of studies ${ }^{113,114,183}$. In one study, the release of a hydrophobic chemotherapeutic agent, 7-ethyl-10-hydroxycamptothecin (SN-38), was prolonged to 30 days by using a triple-layered fiber in which $\mathrm{SN}-38$ was encapsulated in the center layer and surrounded by two superhydrophobic outer layers consisting of PCL and poly(glycerol monostearate-co- $\varepsilon$-caprolactone) ${ }^{177}$. Similar to the trends seen for transient and short-term release from multilayered fibers, increasing the thickness of the outer fiber substantially improved the longevity and amount of drug released. In another study, multilayered fibers comprised of a PCL shell and a PEO/Rhd B core were fabricated to assess the effect of increasing the outer layer thicknesses between 46.1, 68.9, and 186.1 $\mu \mathrm{m}^{184}$. While the thinnest $46 \mu \mathrm{m}$ layers released $85 \%$ of Rhd B in one day, the 68.9 and $186.1 \mu \mathrm{m}$ layers increased release to 15 and 25 days, respectively. Moreover, the release from the two fibers with the thicker outer layers demonstrated zeroorder kinetics, producing gradual, even release of drug with respect to time.

\section{Applications for Intravaginal Delivery}


Multilayered fibers have shown promise as a platform to co-deliver or prolong the release of active agents in different environments. The process of creating multilayered fibers is relatively simple, eliminating the more complex setup and considerations of polymer-solvent interactions between the adjacent, simultaneously spun layers present in coaxial spinning. By removing this complexity of interactions, multilayered fibers can achieve "programmed release" by simply modulating the thickness of each layer.

Multilayered fibers possess other unique features that make them excellent candidates for intravaginal delivery applications. One of the unique strengths of multilayered fibers is that they can provide spatially-specific release in that, unlike other architectures, the individual layers of multilayered fibers can be designed for specific and discrete purposes. For example, one layer may be designed to improve mucoadhesion for enhanced longevity and biocompatibility within the FRT, while another layer may provide release of active agents dependent on its location within the multilayered matrix. Compared to coaxial fibers, the optimization of multilayered fibers is not limited by solvent compatibility, as they can be sequentially spun and assembled post-fabrication. Moreover, multiple individually spun layers can increase the ease of encapsulating multiple types of active agents, which serve mechanistically different roles against a single type of viral infection or as a multipurpose viral-contraceptive or viral-bacterial dosage form. Finally, each fiber layer can be adjusted to have distinct mechanical properties that include tensile strength, porosity, and elasticity, important for comfort and user preference ${ }^{185}$. 
To date, the use of multilayered fibers for intravaginal delivery has been briefly explored ${ }^{104,145,166}$. In one study, circular sheets of pre-spun PVP and PVPEC fibers were stacked and annealed via a pressed metal die that was dipped in solvent. The die annealed the edges of the stacked fibers, creating a multilayered fiber with a PVP inner layer surrounded by blended PVP-EC sheaths. Other multilayered fibers were also constructed by folding the outer layers and pressing the seams. Both types of multilayered fibers encapsulated the hydrophilic compound maraviroc and provided biphasic release, exhibiting an initial burst release followed by short-term release for up to five days. Another study from the same group examined tenofovir (TFV) localization within stacked PCL/PLGA fibers. It was found that TFV localization within the multilayered fiber could be predicted by considering the changes in polymer crystalline structure caused by encapsulant-polymer interactions and correlating drug-polymer hydrophilicity ${ }^{104}$.

Both multilayered and coaxial fibers have the potential to provide tunable and sustained-release; however, each architecture still faces the challenges surrounding FRT delivery. For example, the interplay between two polymer solutions still needs to be considered for interwoven multilayered (and coaxial) fibers, which may result in challenges to altering active agent release. Additionally, as stated previously, the most significant obstacles to intravaginal delivery are providing a dosage form that can facilitate active agent penetration of mucus and retention and release of therapeutically relevant agent concentrations within the FRT. To improve retention, fibers can be fabricated using polymers or polymer blends that have mucoadhesive properties. However, this longevity is rarely 
translated to active agents once they have been released from fibers. Thus, new measures may be considered to provide efficacious and sustained-delivery from fibers.

\section{Composite Nanoparticle-Fiber Delivery Vehicles}

\section{Nanoparticle-Fiber Architectures and Properties}

Over the past two decades, polymeric NPs have been extensively studied as efficacious drug delivery platforms for a variety of applications. Polymeric nanoparticles are an attractive option for intravaginal delivery relative to traditional delivery platforms such as gels and films due to the tunability of active agent release, ability for surface modification, potential for targeted delivery, enhanced distribution potential, and the often resulting enhanced efficacy of encapsulated agents. Additionally, polymeric NPs have been shown to elicit minimal immune response and to improve the delivery and bioactivity of biologics ${ }^{186-188}$. Although metallic nanoparticles have also been explored for use in many drug delivery applications, they have been less commonly administered within the FRT, hence, a more comprehensive review of their applications may be found in ${ }^{189,190}$.

Many physicochemical characteristics of NPs can be altered, such as particle size, surface charge, and hydrophobicity, which contribute to their success in achieving sustained-release and localization to target sites ${ }^{191}$. Although NPs have proven to be effective delivery platforms, as discussed in previous reviews $^{192,193}$, achieving the prolonged release of active agents can be difficult due to the natural clearance mechanisms of the FRT. In particular, NPs are challenged 
with retention in the vaginal cavity due to mucus clearance and transport through mucus to underlying tissue ${ }^{194-196}$. These challenges may be overcome by incorporating NPs into electrospun fibers, thereby creating a composite delivery vehicle that complements the capabilities of both technologies. One might envision that fibers may act as a reservoir for NPs, improving NP and active agent retention, while the innate fiber porosity can help to more finely tune encapsulant release from NPs relative to the release observed from freely administered NPs or fibers.

Nanoparticle-fiber composites are dual-component systems that have the ability to alter the release kinetics of active agents from NPs or NPs themselves ${ }^{197,198}$. Often, the active agent of interest is encapsulated within the NPs, which are then preloaded into polymer solutions for subsequent electrospinning. While a variety of inorganic NPs have been incorporated into fibers $^{199-201}$, concerns still persist regarding the safety of their use relative to polymeric NPs, particularly for intravaginal applications. By utilizing biocompatible polymeric materials for both nanoparticles and fibers, composites may provide safe and prolonged release for clinical applications.

\section{Release Kinetics from Nanoparticle-Fiber Composites}

\section{Transient Release}

Nanoparticle-fiber composites have been used to rapidly release NPs and their encapsulated agents. A study was conducted with hydrophilic PVA and PEO fibers that incorporated PLGA NPs that contained the dye, Coumarin 6202. PEO fibers released $90 \%$ of NPs within 30 min when immersed in a 50:50 ethanol:PBS 
solution, followed by additional release (5\%) after $3.5 \mathrm{~h}$. In comparison, PVA fibers released approximately $70 \%$ of PLGA NPs within 30 min, followed by a decrease in NP release (15\%) over $8 \mathrm{~h}$. Slightly slower release over $24 \mathrm{~h}$ was observed when PVA fibers were crosslinked prior to NP incorporation. This study highlights that nanoparticle-fiber composites can be used to successfully incorporate NPs and to modulate the transient release of NPs from these composites within aqueous solutions 202 .

\section{Short-Term Release}

Several studies have utilized nanoparticle-fiber composites to provide the short-term release of active agents. One group explored a composite drug delivery system that encapsulated the antibiotic, erythromycin, in gelatin NPs and free lidocaine hydrochloride within PVA-chitosan blended fibers ${ }^{203}$. Eighty percent of the lidocaine hydrochloride was released from the fibers within $54 \mathrm{~h}$, while $70 \%$ of the erythromycin was released after $70 \mathrm{~h}$. In contrast, free gelatin NPs released $90 \%$ of erythromycin within the same duration. In a separate study, chitosan-PEO blended fibers containing methoxypolyethylene glycol (mPEG)-b-PLA micelles demonstrated a low initial burst release (15\%) of 5-fluorouracil (5-FU), followed by prolonged release $(91 \%)$ for $109 \mathrm{~h}^{204}$. In another study, the release of free hydrophobic naproxen and chitosan nanoparticles containing Rhd B was studied from PCL fiber scaffolds ${ }^{205}$. Rhodamine B exhibited low levels (5\%) of burst release, while $30-40 \%$ of naproxen was released within the first $2 \mathrm{~h}$. Moreover, after $72 \mathrm{~h}$, only $20 \%$ of Rhd B was released, while $60 \%$ of naproxen was released. The rapid release of naproxen was achieved via incorporation within the fiber 
scaffold, while the extended release of Rhd B was obtained and enhanced through nanoparticle-fiber encapsulation. These results demonstrate the utility of nanoparticle-fiber composites in providing the short-term release of multiple agents.

\section{Sustained-Release}

Nanoparticle-fiber composites have also demonstrated long-term release capabilities in several studies. In one study, dual-release nanoparticle-fiber composites were used to mend and treat critically sized calvarial defects in rats ${ }^{206}$. These composites, consisting of PCL-Co-PEG fibers encapsulating dexamethasone and BSA NPs and loaded with bone morphogenic protein-2 (BMP2), demonstrated sustained-release of both molecules over 35 days. Another study explored the incorporation of siRNA into chitosan NPs and PLGA fiber composites $^{198}$. In these composites, the release of active siRNA was sustained in vitro, with $95 \%$ of siRNA released from the fibers over 32 days, while gene silencing activity was maintained. Sustained-release from nanoparticle-fiber composites was also demonstrated in another study with chitosan-PEO electrospun fibers that were loaded with PLGA NPs encapsulating phenytoin. Nearly complete release of phenytoin from the composite scaffold was achieved over nine days ${ }^{207}$. Lastly, PLA fibers encapsulating chitosan particles provided sustained-release of BSA (45\%) for 27 days, while chitosan particles alone released $80 \%$ BSA in 14 days $^{208}$. 
In addition to NP incorporation within traditional uniaxial or blended fibers, NPs have been incorporated in more complex fiber architectures to prolong the release of active agents. For instance, the effect of combining a multilayered fiber architecture with nanoparticle-fiber composites was investigated by fabricating alternating layers of poly-I-lactic acid (PLLA) and PCL fibers with layers of PCL fibers encapsulating positively-charged chitosan BSA NPs ${ }^{209}$. The multilayered composite released $80 \%$ of the BSA in approximately eight days, whereas the monolayer control released the same concentration of BSA within $24 \mathrm{~h}$.

\section{Applications for Intravaginal Delivery}

Composite delivery vehicles containing nanoparticles and fibers have thus far been primarily studied in wound healing and tissue engineering to fabricate scaffolds for tissue regeneration and bone remodeling ${ }^{133,210-212}$. However, these platforms may be promising candidates for intravaginal delivery applications due to their structural stability and ability to sustain the release of active agents. In such systems, the fibers may be utilized as a reservoir for NPs to aid in intravaginal retention by helping to decrease NP clearance during shedding. In addition, it is envisioned that, depending on fiber formulation and, importantly, NP size and charge, NP (and active agent) release may be modulated, enabling NPs to traverse mucus and deliver agents to target cells that reside in the epithelium or underlying lamina propria. Similar to other architectures, fiber parameters such as polymer composition and size can be tailored to impact release in combination with altering NP composition, size, and loading within the fiber. 
For intravaginal delivery applications, NPs can impart cell specificity, cell internalization, as well as mucoadhesive or mucopenetrative properties to their encapsulated active agents ${ }^{46}$. Numerous studies have demonstrated the ability of NPs to enhance cell targeting via surface modification ${ }^{213,214}$. Additionally, surface modification can increase cell internalization, which may enhance the transport, subcellular localization, and corresponding efficacy of drugs like tenofovir disoproxil fumarate (TDF), which require cell internalization. Furthermore, the NP surface charge can be modulated to provide either mucoadhesive or mucopenetrative properties that further enhance active agent delivery. Additionally, fibers can be fabricated to encapsulate NPs for sustained-release as well as free agents for rapid release, providing both on-demand and sustainedrelease in one platform. Finally, nanoparticle-fiber composites, when coupled with coaxial or multilayered fiber architectures, provide an attractive strategy to retain and sustain the release of active agents within the FRT (Figure 1.7). 
A
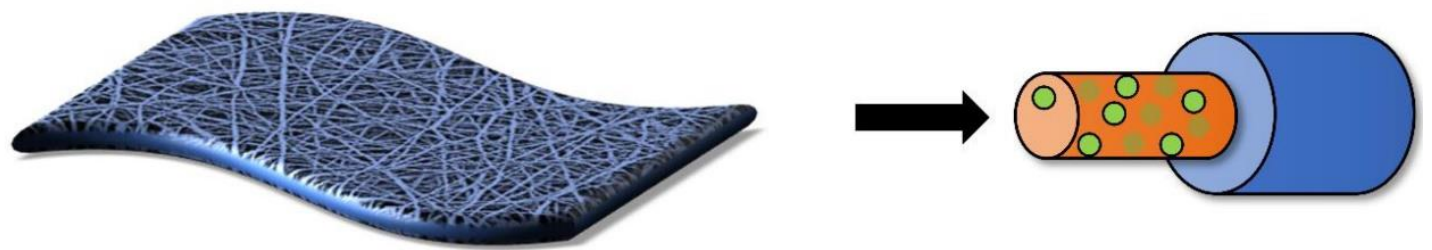

B
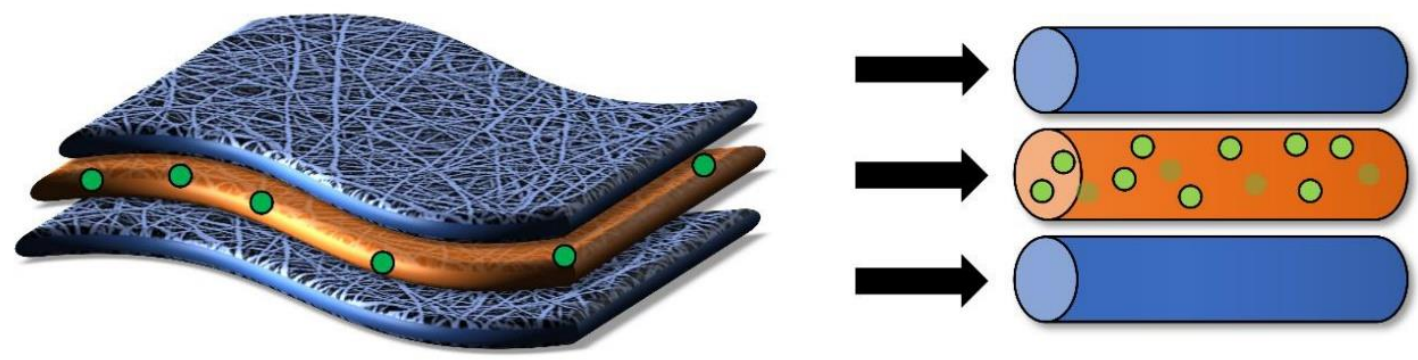

Figure 1.7 Schematic of electrospun nanoparticle-fiber composites that integrate coaxial and multilayered fiber architectures. (A) Coaxial fibers can be fabricated to encapsulate nanoparticles (NPs) within the core fiber, conferring sustained- or delayed-release of active agents that are encapsulated in NPs (shown in green). (B) Multilayered fibers that encapsulate NPs can also act as reservoirs for either NP or active agent release. 
As with multilayered fibers, the use of nanoparticle-fiber composites has only recently been investigated for intravaginal delivery. In a proof-of-concept study, rapid-release PEO, PVA, or PVP fibers encapsulated PLGA NPs containing C6 dye or etravirine drug ${ }^{215}$. In this study, composites and free NPs were administered within murine FRTs and assessed for retention and release. The encapsulated nanoparticles exhibited a 30 -fold increase in retention in the mouse FRTs relative to free NPs. Furthermore, nanoparticles alone provided transient release of etravirine, while all nanoparticle-fiber composites demonstrated release for up to seven days. To date, this is the only investigation of nanoparticle-fiber composites for use in intravaginal delivery. However, the significant difference in retention and release rate achieved with nanoparticle-fiber composites highlights the immense potential of this architecture for sustained-delivery in the FRT.

Although combining nanoparticles and electrospun fibers into one delivery vehicle has demonstrated potential, challenges exist for this platform. The major concern is related to the concentration of nanoparticles that can be effectively encapsulated within fibers without hindering the ability of the polymer solution to be electrospun ${ }^{216}$. Furthermore, the concentration of active agent may decrease with the use of a coaxial or multilayered architecture, as only specific layers of the fiber will encapsulate NPs. Finally, polymeric NPs are often comprised of the same or similar polymers as electrospun fibers, thus care must be taken to prevent polymer solvents from dissolving the NPs prior to or during the electrospinning process $^{217}$. Moreover, the morphology of NPs may also be adversely affected by electrospinning voltage. These factors limit the combinations of fiber and 
nanoparticle materials available for composite fabrication. Thus, for composite delivery applications to succeed, polymer choice and electrospinning conditions must be taken into consideration.

\section{Future Directions and Discussion}

Within the past decade, electrospun fibers have been explored as a multipurpose delivery platform to prevent and treat sexually transmitted infections (STIs). For intravaginal applications, fibers have typically been uniaxially electrospun to release active agents targeted to HIV-1/HSV-2 infections and contraceptive applications. However, other electrospun architectures have been developed that may provide more finely-tuned active agent release, the encapsulation of multiple agents, and longer release durations, desirable for nextgeneration vehicles. Given this, the goal of this review was to summarize the advancements in electrospun fiber architectures including coaxial, multilayered, and nanoparticle-fiber composites, to meet these needs, and to review their use in other drug delivery applications. We sought to relate different temporal regimens of delivery, including transient (occurring within hours), short-term (spanning hours to one week), and sustained (extending from one week to months), to architectural design and materials selection to help guide the design of future platforms that meet the unique temporal needs of intravaginal delivery.

One of the major challenges facing intravaginal delivery is the lack of user adherence surrounding the administration of current delivery platforms. Several clinical trials have highlighted how a lack of user adherence contributes to 
decreased efficacy in clinical trials. In both the FACTS-001 and VOICE trials, South African women deemed high risk for HIV-1 exposure were given antiretroviral TFV gels to administer prior to intercourse ${ }^{29,218}$. Despite the known efficaciousness of TFV, the gels provided suboptimal protection against HIV-1 infection, which was attributed to low user adherence of the gels prior to intercourse. Another study examined the efficacy of gels that incorporated the antiviral polysaccharide, carrageenan, in women in Thailand. This study demonstrated similarly disappointing clinical outcomes, with low user adherence considered the most significant reason for the lack of clinical efficacy ${ }^{219}$. Negative outcomes in other trials such as PRO-2000 and cellulose sulfate gel studies, which examined the efficacy of anti-HIV gels in female populations, further validated these studies, highlighting that both user preference and adherence regimens must be considered during product design rather than at the clinical trial stage. As a result of these studies, there has been an increased emphasis to design vehicles that decrease the administration frequency by prolonging active agent release after a single topical application.

In conjunction with improving user adherence, the development of multipurpose delivery vehicles that offer long-term protection against the various stages of a single infection or a diversity of different types of infections is highly desirable 220 . For single infections, a delivery platform may administer multiple agents with different mechanisms of action that target different stages of the viral or bacterial life cycle. However, the increased likelihood of viral co-infections, such as HSV-2 and HIV-1, as well as bacterial and fungal infections will likely require 
co-administration of antiviral and antimicrobial agents to be successful. Furthermore, applications that seek to meet both antiviral and contraceptive needs in the same dosage form will require the incorporation of multiple types of agents to expand a platform's effectiveness. Therefore, a delivery platform that has the capability to release multiple active agents, each over time frames relevant to the application or active agent, will have greater utility and enable more convenient administration schedules based on specific user needs.

Despite these needs, tailoring the delivery of multiple types of active agents for viral, bacterial, fungal, and contraceptive applications is an ambitious goal given the unique chemical properties of each agent. For example, the antiretroviral TFV and its pro-drug TDF have similar structures and both work as nucleoside reverse transcriptase inhibitors yet possess markedly different hydrophobicities. As such, a delivery platform designed to prolong TFV release may result in different release kinetics of TDF, requiring the formulation of distinct delivery vehicles specific to the selected active agents ${ }^{104,161,162,221,222}$. Furthermore, each active agent may necessitate specific temporal dosing regimens to provide protection or treatment. For example, it may be desirable to administer viral entry inhibitors, which inactivate virions prior to cell entry, over a different time frame than active agents that work inside of cells and need to transport through and localize to target tissue. Several studies have investigated this and have found that more complex and specialized architectures may be useful to achieve temporal delivery goals by tuning the release properties of multiple encapsulants for multiple targets ${ }^{223,224}$. Similarly, for contraceptive applications, on-demand and/or zero-order release with 
equivalent daily dosing may be desirable for spermicides and hormonal/nonhormonal contraceptives, respectively. Conversely, it may be desirable to deliver active agents such as hormones and small hydrophilic drugs (e.g., etonogestrel and acyclovir) within the same time frame for simultaneous long-term contraception and prevention. However, the drastically different chemical properties of these agents will require more complex solutions to achieve similar release profiles. Given this, multipurpose intravaginal delivery platforms must be tailored to maximize the efficacy of individual active agents, including small molecule drugs, proteins, antibiotics, hormones, and live organisms (e.g., probiotics), to meet the needs of these diverse applications.

While providing distinct release profiles of different active agents is an important criterion for the development of future intravaginal platforms, to date, intravaginal rings (IVRs) are the only platforms that provide delivery over a duration of weeks to months ${ }^{225-229}$. Furthermore, IVR studies indicate that more complex dosage forms, such as rings with drug-encapsulating pods, may more likely succeed, particularly in challenging delivery scenarios, e.g., achieving the sustained-release of small hydrophilic molecules ${ }^{223}$. These and other studies $223,224,230,231$ emphasize the need to offer alternative delivery vehicles for women, with the key lesson that platform architecture must be designed to consider the hydrophobicity and chemical compatibility of the encapsulants in combination with its surrounding materials.

In addition to the development of fibers with more complex architectures, active agent release and transport from these platforms must be assessed. Tissue 
mimetics and ex vivo tissues have been used to assess these parameters within the context of intravaginal delivery applications ${ }^{161,232-237}$. One of the most common ways in which to assess intravaginal delivery is by using human ectocervical tissue explants derived from patients ${ }^{233-237}$. These explants provide a representative environment in which to measure transport by accounting for the three-dimensional structure of patient tissue. However, patient-specific variations and tissue availability can limit the use of vaginal explants. Given this, organotypic threedimensional vaginal tissue models such as EpivaginalTM tissue have been created to help evaluate the safety, transport, and efficacy of active agents within an FRT mimetic ${ }^{238,239}$. Other in vitro models have also been developed to explore bacteria and host cell interactions in the reproductive environment ${ }^{240}$. Moreover, within the past decade, new biomarkers and assay endpoints have been identified and studied in different models to more fully assess microbicide interactions with the $\mathrm{FRT}^{241}$. The use of tissue models promises to streamline the assessment of future fiber platforms as viable intravaginal delivery platforms.

To date, a variety of studies have developed uniaxial electrospun fibers for intravaginal applications, including HIV-1 prevention $75,83,85,86,104,160-162,221,222,242,243$. In these studies, electrospun fibers have demonstrated promising potential for intravaginal applications due to their mucoadhesive characteristics, mechanical properties, and ability to be fabricated in different shapes and sizes ${ }^{101}$. Depending upon the polymer hydrophilicity, traditional uniaxial fibers have been formulated as transient, short-term, or long-term delivery platforms. For the purposes of ondemand and short-term release, many of these studies use hydrophilic fibers, 
which dissolve or degrade quickly. In contrast, fibers consisting of more hydrophobic materials are expected to persist within the FRT, acting as reservoirs to sustain the release of active agents. We envision (and have observed) that longterm delivery vehicles maintain their structure during the delivery duration of interest and may require physical removal from the FRT, similar to current IVRs. However, one of the key challenges for intravaginal delivery has been to sustain the release of small hydrophilic antiretrovirals due to their rapid diffusion through the porous fiber matrix, solubility in aqueous solutions, and chemical incompatibility with hydrophobic polymer cores ${ }^{38}$. Many of these uniaxial fibers demonstrated burst release of hydrophilic agents followed by short-term release ${ }^{107,244}$, partially attributed to the localization of hydrophilic agents on the fiber surface. Compounding this, concerns exist that the subsequent release of active agents may be insufficient to provide complete protection against future infections. While blended uniaxial fibers have been moderately successful in addressing these challenges, more work is required ${ }^{85}$.

The primary parameters that impact release from uniaxial fibers are the choice of solvent and polymer. Other factors such as polymer concentration and electrospinning parameters also play a role in attaining different release profiles; however, it is unlikely that these factors alone are sufficient to overcome the challenge of delivering sustained and therapeutically-relevant concentrations of hydrophilic agents. Furthermore, it is difficult to utilize traditional uniaxial fibers for the encapsulation of multiple diverse agents such as large proteins and small 
drugs. Due to these issues, other electrospinning architectures may be better suited to meet the diverse challenges of intravaginal delivery.

As discussed previously, coaxial fibers have shown promise for the encapsulation and release of small hydrophilic and hydrophobic molecules, which may be useful for intravaginal delivery applications. The different goals of transient, short-term and long-term release can be achieved by changing the composition and hydrophobicity of core and shell materials as well as by modulating the shell thickness and core:shell ratio. As described, the shell layer can help regulate active agent release, while the core layer is designed to provide optimal compatibility with an encapsulant. For instance, by using $\mathrm{pH}$-responsive polymer shells, an immediate stimuli-responsive release of agents can be achieved when the fiber is in contact with semen. In this scenario, the core layer may be tailored to encapsulate multiple agents, while the shell, comprised of $\mathrm{pH}$-sensitive polymers, retains encapsulants until needed. Another advantage of coaxial fibers is that they can be fabricated to exploit drug-polymer hydrophilicities. For example, a coaxial fiber comprised of a hydrophobic shell and hydrophilic core can be utilized to provide long-term release of hydrophilic compounds. Agent encapsulation into both layers would allow for both transient burst release from the shell due to surface localization and high loading and sustained-release from the core layer. Finally, coaxial fibers can provide release of biological agents such as large proteins. Coaxial cores may be engineered to achieve high protein encapsulation and biocompatibility, while shells can be constructed with porous surfaces, allowing tunable release. This is particularly significant given that many biologics 
are being investigated as future viral prophylaxes and therapeutics. Although coaxial electrospinning is a more complex process that requires additional optimization, relative to uniaxial spinning, it may enable a versatile platform for transient, short-term, and long-term release ${ }^{85}$.

Multilayered fibers combine different polymer layers via sequential or postspinning to incorporate multiple and chemically distinct drugs within specific layers, thereby tailoring the release kinetics for each encapsulated agent. Multilayered interwoven fibers can be utilized to provide transient release using sacrificial layers to encapsulate agents for on-demand applications. The sacrificial layers comprised of hydrophilic polymers would provide on-demand release of agents based on their immediate degradation when exposed to physiological fluids. Active agent release can be further modulated by the number, thickness, and porosity of each fiber layer ${ }^{245}$. Moreover, blank fibers may be incorporated within the multilayers to either act as a physical barrier for sustained-release or for contraceptive purposes. The layer thickness and level of porosity of blank fibers can be conveniently modulated to delay the release of small hydrophilic molecules from the drug-loaded layers, serving to prolong release. Additionally, multilayered fibers have the potential to deliver biologics and non-hormonal contraceptives. These agents, although efficacious, may degrade when exposed to harsh solvents during the electrospinning process. By incorporating these active agents in distinct layers and integrating barrier layers, multilayered fibers can provide long-term release of drugs and biologics while retaining their activities. 
While each of these strategies offers advantages relative to uniaxial spinning, the delivery of active agents may be further enhanced by integrating nanoparticles with fibers. A composite platform may offer a new alternative to address the challenges of intravaginal delivery, such as the maintaining active agent stability, providing cell-specific targeting (via NPs), and enhancing cell internalization. Like electrospun fibers, nanoparticles can be designed to encapsulate virtually any compound. The limitations of nanoparticle-fiber composites mentioned earlier may be overcome by utilizing fibers as a reservoir for both active agents and nanoparticles to release multiple therapeutics. Furthermore, the release rates of encapsulants from both nanoparticles and fibers may be modulated by adjusting the composition of the polymeric scaffold. For ondemand transient release, hydrophilic polymers may be used to enable rapid release of NPs for immediate distribution through and enhanced retention within tissue. In contrast, more hydrophobic fibers may be used to delay the release of NPs or NP-encapsulated agents. Although drug-polymer hydrophobicity is a major contributor to release, other factors such as polymer choice, molecular weight, and crystallinity, as well as solvent choice and electrospinning parameters, also affect the release of agents from fibers.

The application of advanced fiber architectures has only recently been explored in the context of intravaginal delivery. Advanced fiber architectures demonstrate the potential to provide the sustained-release of individual active agents in addition to concurrently providing both transient and sustained-delivery of multiple active agents. These are key advantages over traditional uniaxial fibers, 
which are challenged with the long-term delivery of small hydrophilic molecules, in addition to providing transient and sustained-release simultaneously. We envision that future fiber architectures will localize active agents within specific sections of the fiber to tailor the release of individual agents independent of other encapsulants. Moreover, we anticipate that future platforms will combine architectures to maximize or complement the advantages of individual platforms. As previous clinical trials have shown, effective protection will be dependent upon fulfilling user preferences, offering convenience, and providing necessary release profiles from one vehicle, which fibers have the potential to realize.

Building upon this previous research, the goal of this work was to employ EFs to topically deliver antiviral agents and provide dual-protection against both HIV-1 and HSV-2 infections. Towards this end, fibers were fabricated to incorporate and provide release ( $>1$ week) of TDF. Additionally, we began to develop delivery vehicles to deliver the antiviral biologic GRFT, using a variety of uniaxial fiber types. These included fibers that were covalently surface-modified with GRFT to serve as "viral traps", and pH-responsive GRFT fibers, enabling ondemand protection against infections. In addition, hydrophilic rapid-release polymer formulations were fabricated to high incorporate GRFT, to provide ondemand protection against viral infections. Finally, EFs and nanoparticles were combined to create a NP-EF composite, incorporating the attributes of both technologies. It was hypothesized that fibers would serve as long-term NP reservoirs to modulate the release of GRFT from incorporated GRFT NPs. 
All fiber formulations incorporating either TDF or GRFT were assessed for loading and (where applicable) release characteristics. All formulations were tested against both HIV-1 and HSV-2 infections in vitro, and the potential cytotoxicity of all formulations was evaluated using MTT assays. Finally, several animal studies were conducted using 5-week-old female BALB/c mice to assess selected fiber formulations for GRFT delivery. First, the in vivo safety of blank fiber formulations was assessed. Histology and cytokine expression were evaluated from collected mouse reproductive tracts and vaginal lavages from mice administered fibers or composites for either 24 or $72 \mathrm{hr}$. Finally, it was hypothesized that these platforms would provide in vivo protection against viral infection, and demonstrate comparable antiviral activity relative to free GRFT. In these studies, mice were administered GRFT fibers or NP-EF composites, followed by a single challenge later with HSV-2 (LD90) after $24 \mathrm{hr}$ platform incubation. Following viral challenge, mice monitored for 14 days post-infection to evaluate survival and possible viral progression. 


\section{CHAPTER 2}

MULTIPURPOSE TENOFOVIR DISOPROXIL FUMARATE ELECTROSPUN FIBERS FOR THE PREVENTION OF HIV-1 AND HSV-2 INFECTIONS IN VITRO

\section{Introduction}

In this study, the goal of our work was to develop PLGA and poly(DL-lactideco- $\varepsilon$-caprolactone) (PLCL) EFs containing TDF to demonstrate safe and efficacious inhibition of both HIV-1 and HSV-2 infections in vitro. TDF was selected as a model ARV to demonstrate proof-of-concept of our delivery vehicles, as at the time of this study, it was a next-generation, more lipophilic form of TFV, that had demonstrated strong protection after sustained-release from IVRs. Here we fabricated both PLGA and PLCL EFs to evaluate and compare two different biodegradable polymers known to impart the sustained-release of active agents. We synthesized 3 different formulations for each polymer, PLGA and PLCL, and characterized the loading and sustained-release of TDF from EFs. We subsequently assessed the efficacy of fiber release eluates against both HSV-2 and HIV-1 infections in vitro, while demonstrating EF biocompatibility in vaginal keratinocytes, ectocervical and endocervical cells, and EpiVaginal tissue.

\section{Materials and Methods}

\section{Materials}


Poly(lactic-co-glycolic acid) (PLGA 50:50, 0.55-0.75 dL/g, 31-57k MW) and poly(DL-lactide-co- $\varepsilon$-caprolactone) (PLCL 80:20, $0.75 \mathrm{dL} / \mathrm{g}, 37 \mathrm{k} \mathrm{MW)} \mathrm{were} \mathrm{both}$ purchased from Lactel Absorbable Polymers (Cupertino, CA). Solvents 1, 1, 1, 3, 3, 3-hexafluoro-2-propanol (HFIP) and trifluoroethanol (TFE) were obtained from Fisher Scientific (Pittsburgh, PA). TDF was purchased as Viread® (Tenofovir disoproxil fumarate, Gilead Sciences Inc., Foster City, CA) tablets from the University of Louisville Pharmacy. Other chemicals, including dimethyl sulfoxide (DMSO), acetonitrile, trifluoroacetic acid and thiazolyl blue tetrazolium bromide (MTT) were purchased from Sigma Aldrich (St Louis, MO). Fetal bovine serum (FBS), antibiotics (penicillin/streptomycin and gentamicin), minimum essential medium (MEM, Corning), keratinocyte serum-free medium (KSFM, Gibco), and Dulbecco's modified Eagle medium (DMEM, Invitrogen) were all purchased from VWR and Thermo-Fisher. Simulated vaginal fluid (SVF) was prepared in house using a previously established protocol ${ }^{246}$. Finally, pure TFV and TDF were kindly provided by the NIH AIDS Reagent Program.

\section{Cell Lines, Virus, and Tissue Culture}

TZM-bl cells were obtained from the NIH AIDS Reagent Program. These cells are a genetically engineered HeLa cell clone that express CD4, CXCR4, and CCR5 and contain Tat-responsive reporter genes for firefly luciferase (LuC) and Escherichia coli $\beta$-galactosidase under regulatory control of an HIV-1 long terminal repeat $^{247,248}$. TZM-bl cells were maintained in DMEM containing $10 \%$ heatinactivated FBS, $25 \mathrm{nM}$ HEPES, and $50 \mu \mathrm{g} / \mathrm{mL}$ gentamicin, in a vented T-75 culture flask. Env-pseudotype HIV-1 was kindly provided by Dr. Nobuyuki Matoba from 
the University of Louisville, and originally obtained from the NIH AIDS Reagent Program. To conduct HSV-2 plaque assays, African green monkey kidney cells (Vero E6, originally obtained from ATCC), HEK-293T (human embryonic kidney cells originally purchased from ATCC) and HSV-2 (4674) were kindly provided by Dr. Kenneth Palmer from the University of Louisville. Cells were maintained in MEM supplemented with $10 \% \mathrm{FBS}$, and $1 \%$ penicillin and streptomycin $(100 \mu \mathrm{g} / \mathrm{mL}$ each).

To assess cytotoxicity, endocervical, End1/E6E7 (End1); ectocervical, Ect1/E6E7 (Ect1); and vaginal keratinocyte, VK2/E6E7 (VK2) cell lines were used (courtesy of Dr. Kenneth Palmer, originally from ATCC). These cell lines were selected because they are representative of the cell types in the female reproductive tract that would be exposed to the topical EFs. End1, Ect1, and VK2 cells were maintained in KSFM supplemented with bovine pituitary extract (50 $\mu \mathrm{g} / \mathrm{mL})$, epidermal growth factor $(0.1 \mathrm{ng} / \mathrm{mL})$, and $1 \%$ penicillin and streptomycin. The media was further supplemented with calcium chloride $(\mathrm{CaCl} 2)$ to a final concentration of $0.4 \mathrm{mM}$. During cell trypsinization for plating and cell count, cells were neutralized using DMEM/F12 (Gibco) with 10\% FBS, and 1\% penicillin and streptomycin. Organotypic EpiVaginal cultures of normal human vaginalectocervical epithelial cells were purchased from and cultivated as suggested by MatTek.

\section{Synthesis of Electrospun Fibers}


PLGA and PLCL EFs were prepared with different solvents and TDF concentrations spanning (1-20\% wt drug/wt polymer (w/w)). Powder from crushed Viread tablets (700 mg tablets containing $300 \mathrm{mg}$ TDF) were used as the source of TDF. The presence of inactive excipients in these samples was accounted for when determining the theoretical loading of TDF into polymer fibers. Blank fibers containing no TDF were prepared as negative controls. For blank EFs, 15-20\% PLGA w/w and $12-20 \%$ PLCL w/w were dissolved in either $3 \mathrm{~mL}$ TFE or HFIP solvent overnight while shaking at $37^{\circ} \mathrm{C}$. The following day, $2 \mathrm{~mL}$ of PLGA or PLCL solution was aspirated into, and electrospun from, a $3 \mathrm{~mL}$ plastic syringe as previously described ${ }^{75,87}$. All formulations were electrospun with a flow rate of 2.0 $\mathrm{mL} / \mathrm{h}$ and an applied voltage of $20 \mathrm{kV}$. EFs were collected on a rotating $4 \mathrm{~mm}$ outer-diameter stainless steel mandrel, located $20 \mathrm{~cm}$ from the blunt needle tip. Sample flow rate was monitored by an infusion pump (Fisher Scientific, Pittsburgh, PA) and the voltage was applied using a high voltage power supply (Spellman CZE 1000R). For fibers incorporating TDF, either 1,10 , or $20 \%$ w/w TDF was dissolved in $1.2 \mathrm{~mL}$ solvent overnight. The next day the TDF solution was added to $1.8 \mathrm{~mL}$ polymer solution prior to electrospinning. After electrospinning, fibers were removed from the mandrel and dried overnight in a desiccator cabinet.

\section{Electrospun Fiber Size and Morphology}

The impact of various parameters including: solvent choice, polymer composition, and TDF concentration on fiber size and morphology were evaluated using scanning electron microscopy (SEM). Desiccated EFs were placed on carbon tape, sputter coated with gold, and imaged using SEM (Supra 35 SEM 
Zeiss). SEM images were acquired at magnifications ranging from $1000-5000 \times$ to enable clear visualization of the fiber microstructure. The average fiber diameter was determined by analyzing SEM images in NIH ImageJ, and drawing line elements across a minimum of 50 fibers per image. Statistical significance between fiber diameters was determined using the Bonferroni post hoc t-test $(p<$ $0.05)$.

Fiber Characterization: Loading, Encapsulation Efficiency, and Controlled Release

Incorporated TDF was quantified via HPLC-UV/Vis using a modified established method ${ }^{85}$. Briefly, $10 \mathrm{mg}$ of PLGA and PLCL fibers were dissolved in $1 \mathrm{~mL}$ of DMSO prior to analysis. Dilutions of these samples were injected into a Waters 515 HPLC pump using a Waters 717 Plus auto sampler with a Waters 2487 absorbance detector. The mobile phase was comprised of an isocratic mixture of $72 \%$ Milli-Q water with $0.045 \%$ trifluoroacetic acid and $28 \%$ acetonitrile with $0.036 \%$ trifluoroacetic acid. The column used for this procedure was a Waters Sun Fire C18 Column, $(100 \AA, 5 \mu \mathrm{m}, 4.6 \mathrm{~mm} \times 250 \mathrm{~mm})$. The instrument method comprised a $1 \mathrm{~mL} / \mathrm{min}$ flow rate, $15 \mathrm{~min}$ run time, UV/vis detection at $259 \mathrm{~nm}$, and $20 \mu \mathrm{L}$ sample injection volume. TFV was found to elute from the column $2.2 \mathrm{~min}$ after injection, while TDF eluted 12 min post-injection. The initial quantification of fibers was performed using combined TDF and TVF standards prepared in DMSO. Standard curves of both TDF and TFV $(0.7-100 \mu \mathrm{g} / \mathrm{mL})$ were used to quantitate incorporated TDF and to assess TDF degradation. Samples from Viread tablets were assessed to verify TDF concentration and were used as standards in 
subsequent experiments. Controls included blank fibers and fibers spiked with a known concentration of TDF. TDF values determined from HPLC measurements were compared with the quantity of TDF added prior to electrospinning to obtain percent encapsulation efficiency (EE), where $E E=[($ Mass of TDF Incorporated)/(Mass of TDF Initially Added)] x 100. Unless otherwise noted, all samples were analyzed in triplicate.

Controlled release experiments were performed to assess the release of TDF from EFs. Triplicate $10 \mathrm{mg}$ fiber pieces were cut and suspended in $1 \mathrm{~mL}$ of simulated vaginal fluid (SVF) to represent intravaginal conditions in vitro. Samples were incubated at $37^{\circ} \mathrm{C}$ and constantly shaken. The complete volume of SVF was removed and replaced with fresh SVF at time points: $1,2,4,6,24,48,72 \mathrm{~h}$, and $1,2,3$, and 4 wk. The amount of TDF in the supernatant was measured using HPLC. Quantification was performed using a Viread standard diluted in SVF, with eluate from blank fibers in SVF used as background correction. Statistical significance of both loading and release profiles between fiber formulations was determined by one-way ANOVA with the Bonferroni post hoc t-test $(p<0.05)$.

\section{In vitro efficacy of PLGA and PLCL fibers against HIV-1 infection}

HIV-1 pseudovirus assays were used to assess the efficacy of TDF released from EFs against HIV-1 infection in vitro. TZM-bl cells were infected with Env-pseudotype HIV-1, kindly provided by both Dr. Nobuyuki Matoba (University of Louisville) and the NIH AIDS Reagent Program. To produce and propagate HIV1 Env-pseudovirus, HEK293T/17 cells were transfected with two plasmids, one 
containing an Env-defective HIV-1 genome and a plasmid solely expressing Env. Transfection was facilitated with the use of FuGENE (Promega). HEK293T cells were allowed to incubate for $48 \mathrm{~h}$, after which viral particles were collected and titered using the 50\% Tissue Culture Infectious Doses assay (TCID50). Viral particles were stored at $-80^{\circ} \mathrm{C}$ until use $e^{249}$.

To determine the in vitro efficacy of PLGA and PLCL TDF EFs against Envpseudotype HIV-1 infection, TZM-bl cells were seeded in 96-well plates at 100,000 cells/well in $100 \mu \mathrm{L}$ of DMEM. Fifty microliters of fiber eluate media (DMEM $10 \%$ FBS) collected from time points: 1 and $24 \mathrm{~h}$; week 1 (release from days $0-7$ ); week 2 (release from days 8-14), week 3 (release from days 15-21), and week 4 (release from days 22-28) were diluted by a maximum of 5 orders of magnitude from collected eluate (1:100,000 maximum dilution). Eluate dilutions were added to cells in triplicate, and $50 \mu \mathrm{L}$ of diluted virus stock (1:8) was subsequently added to each well. The administered virus dose resulted in relative luminescence units $(R L U)$ of at least twenty times that of background observed in untreated/uninfected cells, yielding an average of 100,000 RLUs in our experiments. Experimental controls included untreated/uninfected cells, untreated/infected cells, and blank fiber eluate-treated/infected cells. For wells containing untreated/uninfected and untreated/infected cell controls, $100 \mu \mathrm{L}$ DMEM was added to the wells; for infected cells with blank fiber eluate, $50 \mu \mathrm{L}$ DMEM was added to $50 \mu \mathrm{L}$ blank fiber eluate, resulting in a final volume of $200 \mu \mathrm{L}$ for all wells. After infection, plates were incubated $48 \mathrm{~h}$ at $37^{\circ} \mathrm{C}$, and $100 \mu \mathrm{L}$ of media was subsequently removed from each well (post-incubation), and replaced with $100 \mu \mathrm{L}$ of Bright Glo Reagent 
(Promega). Cells were incubated at room temperature for another $5 \mathrm{~min}$ and the luminescence of each well was read at an integration time of $1 \mathrm{~s}$ and a gain of 135 (Synergy HT luminometer). The amount of virus inhibition was determined by normalizing the RLUs of treated/infected cells to untreated/infected cells. Additionally, all RLU values were corrected by subtracting the RLU of untreated/uninfected cells. $\mathrm{IC}_{50}$ values were determined using GraphPad 6.0 sigmoidal regression analysis. Unless otherwise noted, all experiments were run with three or more replicates per treatment group. Statistical significance between the $\mathrm{IC}_{50}$ s was determined using one-way ANOVA with the Bonferroni post hoc ttest $(p<0.05)$.

\section{In Vitro Efficacy of PLGA and PLCL Fibers against HSV-2 Infection}

HSV-2 plaque assays were conducted to test the efficacy of TDF EFs against HSV-2 infection in vitro. Fibers were incubated in $10 \mathrm{~mL}$ complete plating media (1\% FBS MEM) for 1 and $24 \mathrm{~h}$. Additional fiber eluates were collected at week 1 (release from days 0-7); week 2 (release from days 8-14); week 3 (release from days 15-21); and week 4 (release from days 22-28) to assess the ability of PLGA and PLCL TDF EFs to provide prolonged delivery and corresponding HSV2 protection. The antiviral activity of PLGA and PLCL TDF EF eluates was determined using HSV-2 (4674) plaque assays in Vero E6 cells. Vero E6 cells were seeded with 600,000 cells/well and grown to near confluence for $24 \mathrm{~h}$ in a 6 -well flat bottom plate. After $24 \mathrm{~h}$, the media was removed and cells were simultaneously administered $2 \mathrm{~mL}$ of fiber eluate serial dilutions from the above collected time points and 3000 PFU of HSV-2 per well. Free TDF was used as a positive control 
for HSV-2 inhibition, in parallel with untreated/uninfected cells; whereas untreated/infected cells were used as a positive control of cell infection and death. After $48 \mathrm{~h}$, cells were fixed with methanol for $10 \mathrm{~min}$, stained with $0.1 \%$ crystal violet for $30 \mathrm{~min}$, and washed with DI water. Plaques were counted, and plaque numbers from experimental groups were normalized relative to the number of plaques in untreated/infected cells ( $280-300$ plaques). Samples were analyzed in triplicate, and GraphPad was used to determine the $\mathrm{IC}_{50}$ values of the TDF EF formulations. Statistical analysis was performed by comparing the average percent inhibition of HSV-2 using one-way ANOVA with the Bonferroni post hoc t-test $(\mathrm{p}<$ $0.05)$.

\section{In Vitro Cytotoxicity of PLGA and PLCL Fibers}

Vaginal epithelial (VK2/E6E7), ectocervical (Ect1/E6E7), and endocervical (End1/E6E7) cells were incubated with TDF EFs in KSFM to assess the in vitro biocompatibility of TDF fibers. Cells were plated at a density of 300,000 cells/well in 12-well plates and incubated in triplicate with $10 \mathrm{mg}$ fiber pieces placed in transwell inserts (10 mg/mL final concentration). No treatment (media alone) and $10 \%$ DMSO were used as positive and negative controls of cell viability, respectively. After 24,48 , and $72 \mathrm{~h}$ incubation, $10 \mathrm{~mL}$ of MTT reagent was added to the cells, cells were lysed, and absorbance was read at $570 \mathrm{~nm}$ the following day. PLGA and PLCL EF-treated cell absorbance values were normalized to untreated cell absorbance to obtain percent viability.

\section{EpiVaginal Cytotoxicity of PLGA Fibers}


Full thickness vaginal epithelial (VEC-100 FT) EpiVaginal ${ }^{\mathrm{TM}}$ tissues (MatTek) were administered low $(5 \mathrm{mg} / \mathrm{mL})$ and high $(50 \mathrm{mg} / \mathrm{mL})$ concentrations of PLGA TDF fibers to best represent administration in a future in vivo model. PLGA samples were chosen due to our initial experiments demonstrating its enhanced TDF release profile and efficacy relative to PLCL. Control samples included untreated, blank PLGA fiber-treated, and toxic (0.2\% nonoxynol-9)treated control groups. The tissues were incubated at $37^{\circ} \mathrm{C}, 5 \% \mathrm{CO}_{2}$ for 2 and 3 days.

To monitor the tissue viability following exposure to TDF PLGA EFs, the basal side tissue culture media was collected on days 2 and 3 . Cytotoxicity was measured using a lactate dehydrogenase (LDH) cytotoxicity assay kit (Pierce). The viability of the TDF EF treated tissues was determined by normalizing the absorbance of the treated tissues to the absorbance of the untreated tissue. Percent cell viability was expressed as: $\%$ Viability $=[\mathrm{OD}$ (treated tissue)/OD (untreated tissue)] $\times$ 100. Transepithelial electrical resistance (TEER) was measured using an EVOM2 Epithelial Voltohmmeter equipped with an Endohm electrode chamber (World Precision Instruments, Sarasota, FL) on days 0, 1, 2, and 3 of the treatment.

To assess inflammatory markers resulting from fiber exposure, tissue media was analyzed for cytokine production. Based on previous work ${ }^{238,239,250,251}$, cytokines: IL-1 $\alpha$, IL- $\beta$, IL-6, IL-8, and TNF- $\alpha$ were assessed in all collected media via Luminex assay. Additionally, GM-CSF, IFN- $\mathrm{y}$, and MCP-1 expression were assessed based on previous microbicide studies using Luminex ${ }^{68,69,252}$. Cytokine 
expression in samples was compared with untreated EpiVaginal tissue via fold increase. The fold-increase was calculated by dividing the sample expression level by the untreated tissue values.

To visually examine the structural integrity of the tissue after 3 days of consecutive treatment, the tissue samples were washed with PBS and fixed with $4 \%$ paraformaldehyde. The tissue specimens were embedded in a paraffin block, stained with hematoxylin and eosin (H\&E), and cross-sections were observed under 20x magnification using an Aperio Imagescope (Leica Biosystems Inc., Buffalo Grove, IL). Tissue samples were subjected to histological analysis by a pathologist blinded to treatment group assignment.

\section{Results}

\section{Electrospun Fiber Size and Morphology}

Fiber morphology was evaluated using SEM, and NIH ImageJ software was used to assess fiber diameters. Blank PLGA and PLCL fibers fabricated using either HFIP or TFE solvent are shown in Figure 2.1. For fibers electrospun in HFIP, $15 \% \mathrm{w} / \mathrm{w}$ PLGA or $12 \% \mathrm{w} / \mathrm{w}$ PLCL provided well-defined fiber morphologies. However with TFE, both polymers required an increase in concentration to $20 \%$ $(w / w)$ to produce well-delineated microstructures. The average diameters were 2.0 \pm 0.8 and $1.7 \pm 0.4 \mu \mathrm{m}$ for $15 \%$ PLGA and $12 \%$ PLCL fibers made with HFIP, and $1.9 \pm 0.9$ and $1.9 \pm 0.8 \mu \mathrm{m}$ for $20 \%$ PLGA and $20 \%$ PLCL fibers made with TFE (Table 2.1). Once well-delineated fibers were established, the effect of TDF 
incorporation on PLGA and PLCL EF morphologies electrospun with HFIP was evaluated (Figure 2.2). 


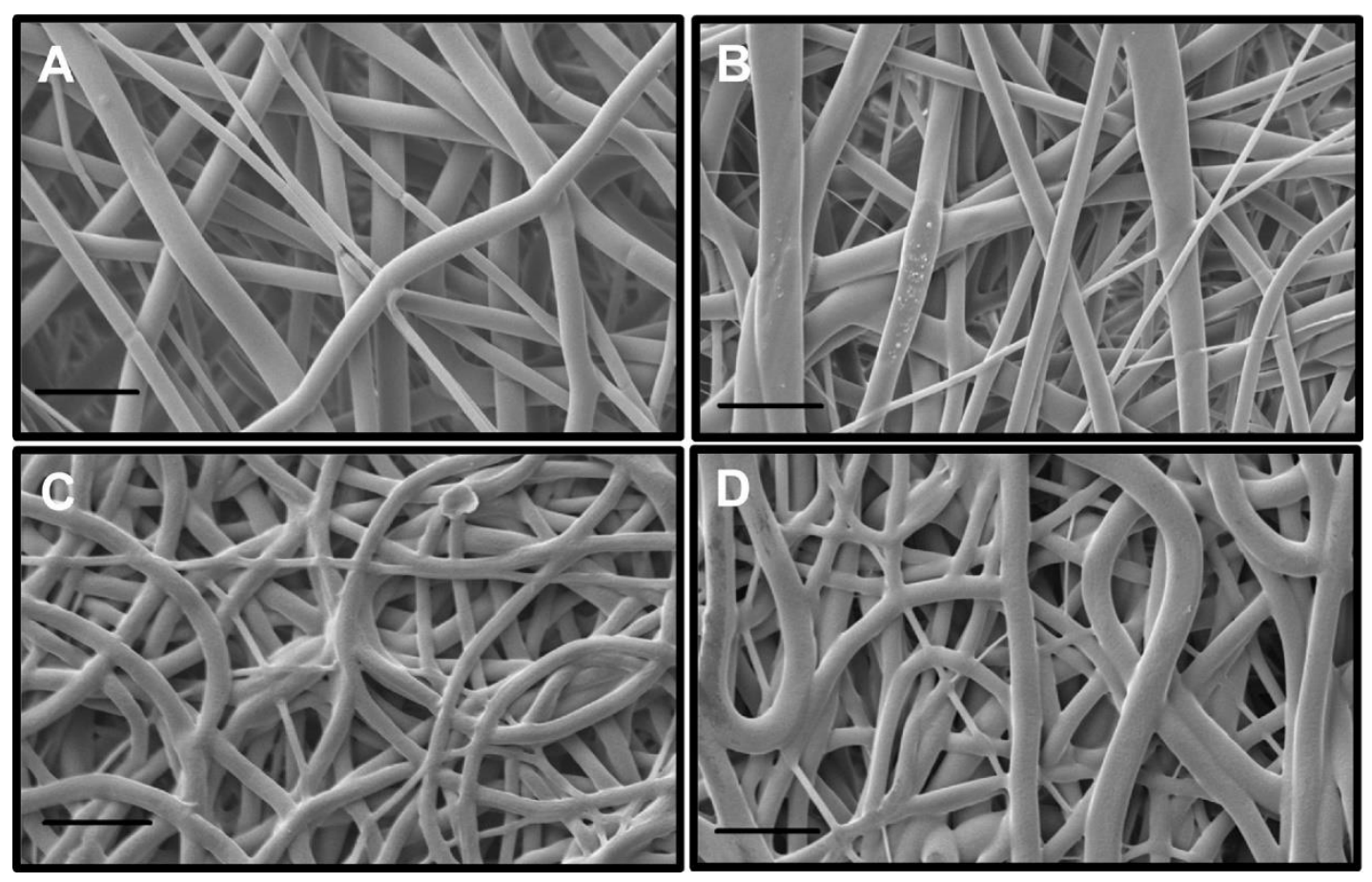

Figure 2.1 SEM images of blank PLGA and PLCL fibers electrospun using different solvents. (A) 15\% w/w PLGA in HFIP; (B) 20\% w/w PLGA in TFE; (C) 12\% w/w PLCL in HFIP; and (D) 20\% w/w PLCL in TFE. Scale bars represent 10 $\mu \mathrm{m}$. 
Table 2.1 Diameters of electrospun fibers. Blank and TDF fibers were fabricated using HFIP or TFE solvents. EFs incorporating TDF exhibited decreased diameters compared with blank fibers.

\begin{tabular}{|c|c|c|}
\hline \multicolumn{2}{|c|}{ Fiber Formulation } & Average Width $(\mu \mathrm{m})$ \\
\hline \multirow{3}{*}{ HFIP 15\% PLGA } & Blank Fiber & $1.7 \pm 0.6$ \\
\cline { 2 - 3 } & $\mathbf{1 \%}$ TDF & $1.1 \pm 0.3$ \\
\cline { 2 - 3 } & $\mathbf{1 0 \%}$ TDF & $0.8 \pm 0.3$ \\
\cline { 2 - 3 } & $\mathbf{2 0 \%}$ TDF & $1.1 \pm 0.4$ \\
\hline \multirow{3}{*}{ HFIP 12\% PLCL } & Blank Fiber & $1.7 \pm 0.5$ \\
\cline { 2 - 3 } & $\mathbf{1 \%}$ TDF & $1.1 \pm 0.5$ \\
\cline { 2 - 3 } & $\mathbf{1 0 \%}$ TDF & $0.9 \pm 0.3$ \\
\cline { 2 - 3 } & $\mathbf{2 0 \%}$ TDF & $0.7 \pm 0.2$ \\
\hline TFE 20\% PLGA Blank Fiber & $2.0 \pm 1.0$ \\
\hline TFE 20\% PLGA 10\% TDF & $1.2 \pm 0.4$ \\
\hline TFE 20\% PLCL Blank Fiber & $1.9 \pm 0.9$ \\
\hline TFE 20\% PLCL 10\% TDF & $0.6 \pm 0.2$ \\
\hline
\end{tabular}




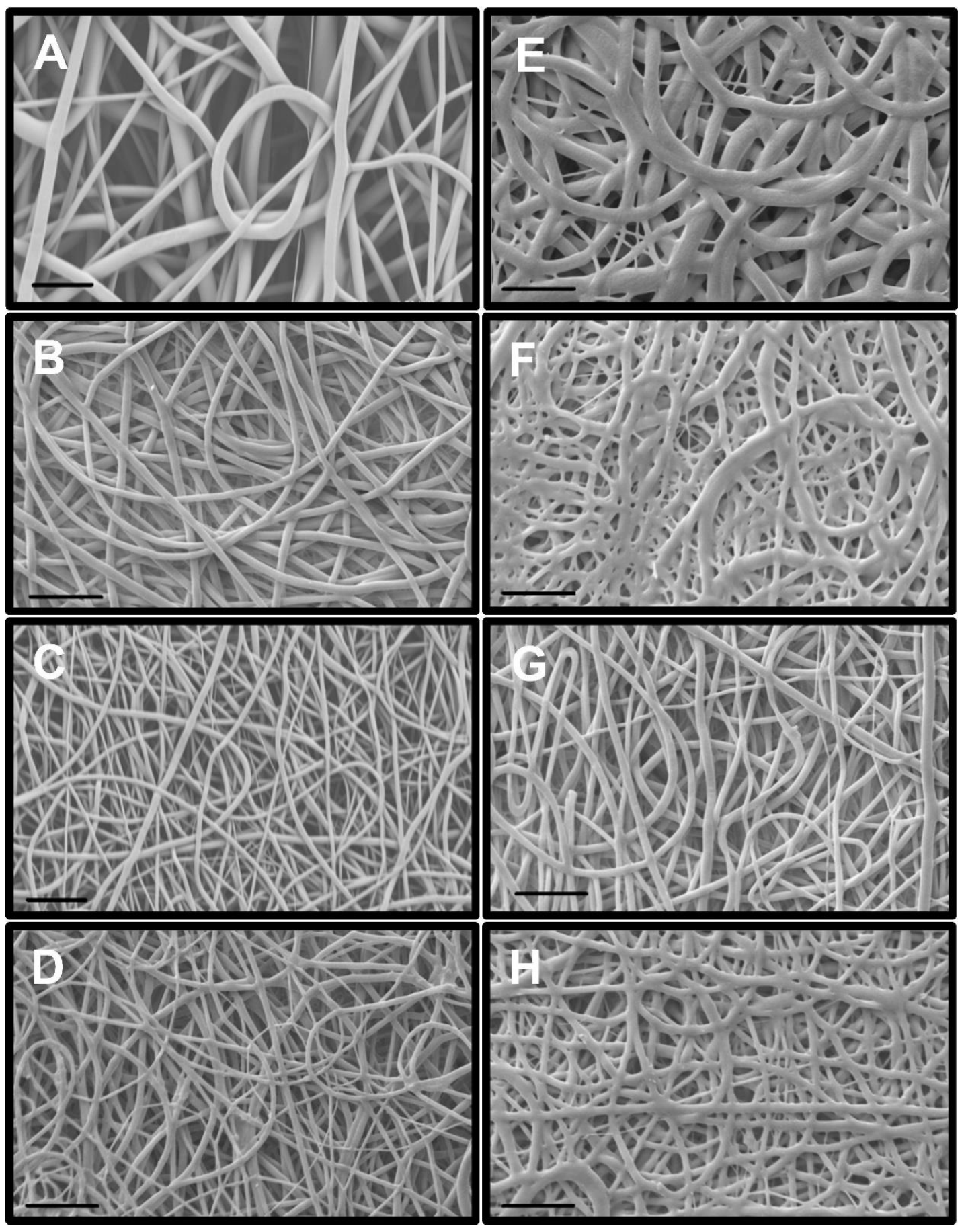

Figure 2.2 SEM images of PLGA and PLCL fibers prepared with increasing concentrations of TDF, using HFIP as the solvent. (A) Blank PLGA, (B) 1\% TDF, (C) $10 \%$ TDF, and (D) $20 \%$ TDF PLGA fibers; (E) Blank PLCL, (F) $1 \%$ TDF, (G) 10\% TDF, and $(\mathrm{H})$ 20\% TDF PLCL fibers. Scale bars represent $10 \mu \mathrm{m}$. 
The morphologies and diameters of TDF EF formulations are shown in Figure 2.2, Figure 2.3, respectively. Prior to TDF incorporation, the average diameters of all blank fiber formulations were similar, ranging from 1.7 to $2.0 \mu \mathrm{m}$, with no statistically significant differences observed between formulations. In comparison, PLGA fibers incorporating TDF showed no particular trend in fiber diameters; whereas PLCL fiber diameters decreased with increased TDF concentration. The average fiber diameters for HFIP 15\% PLGA $1 \%, 10 \%$, and $20 \%$ TDF were $1.1 \pm 0.3,0.8 \pm 0.3$, and $1.1 \pm 0.4 \mu \mathrm{m}$. For HFIP $12 \%$ PLCL $1 \%$, $10 \%, 20 \%$ TDF fibers, the resulting fiber diameters were $1.1 \pm 0.5,0.9 \pm 0.3,0.7 \pm$ $0.2 \mu \mathrm{m}$ (Table 2.1). TDF-incorporated fibers electrospun with TFE solvent, displayed a similar decrease in diameters to $1.2 \pm 0.4 \mu \mathrm{m}$ and $0.6 \pm 0.2 \mu \mathrm{m}$, for PLGA and PLCL respectively. All TDF fibers exhibited statistically significant decreases in fiber diameter relative to blank PLGA (1.7 and $2.0 \mu \mathrm{m}$ for HFIP and TFE blank EF respectively) and PLCL (1.7 and $1.9 \mu \mathrm{m}$ for HFIP and TFE blank EF respectively) EFs. However, no statistical significance was observed between the 1,10 , and $20 \%$ TDF fiber formulations as a function of TDF incorporation. Thus, TDF incorporation resulted in decreased fiber diameter relative to blank fibers; whereas variation in the amount of TDF incorporation had no significant effect on fiber diameter. 

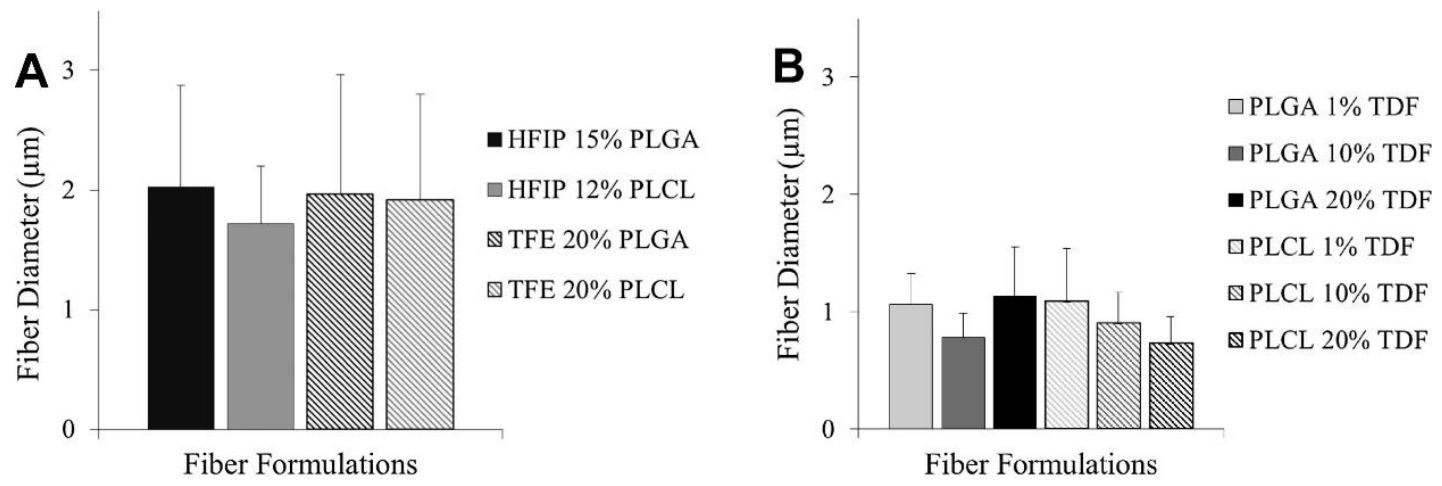

Figure 2.3 Average diameters of electrospun fibers measured from SEM images, using ImageJ. (A) Diameters of blank PLGA and PLCL fibers electrospun with either HFIP or TFE solvents. Diameters ranged from 1.7 to $2.0 \mu \mathrm{m}$. Statistical significance between fiber diameters was determined using one-way ANOVA with Bonferroni post hoc test ( $p<0.05, n=150,3$ images, 50 measurements per image). No statistical significance was observed between fiber diameters prepared with either HFIP or TFE. (B) Diameters of TDF EFs electrospun with HFIP were significantly smaller than those of blank fibers, ranging from 0.7 to $1.2 \mu \mathrm{m}$. While the PLGA TDF fiber diameters seemed randomly distributed, PLCL TDF fibers demonstrated a trend of decreased diameter with increasing TDF concentration. Statistical significance was observed between blank fibers and all TDF fiber diameters; however, no statistical difference in diameters was observed between the TDF EF formulations. 
Fiber Characterization: Loading, Encapsulation Efficiency, and Controlled Release

To determine the loading of TDF in PLGA and PLCL fibers, different concentrations of TDF $(1,10$, and $20 \% \mathrm{w} / \mathrm{w})$ were incorporated. The TDF, TFV, and Viread standard curves, used to quantify TDF in fibers, maintained linearity and similar peak intensities in both DMSO as well as SVF (Supplemental Figure 2.1). For all samples examined, TDF incorporated into fibers remained stable against degradation or hydrolysis. Although TFV peaks were present in many loading samples, they were either below the limit of quantification or comprised less than $2 \%$ of TDF sample concentration. In addition to TFV peaks, a minor peak eluting at 3.5 min was present in all samples and standards containing TDF. This peak, dubbed "minor TDF" comprised an area that was $4 \%$ of the TDF peak area (Supplemental Figure 2.1) and may be attributed to the monoester derivative of TDF (mPTFV). This proportionality was observed in all loading samples as well as controlled release samples collected during the first week of release. After 1 wk, the proportion of mPTFV increased, reaching a 1:1 ratio with TDF in some samples (data not shown). This increased ratio of MPTFV:TDF is attributed to the increased exposure of fibers to aqueous solution at later time points, coupled with an overall decrease in TDF release. The mPTFV concentration was quantified using the TDF standard. 

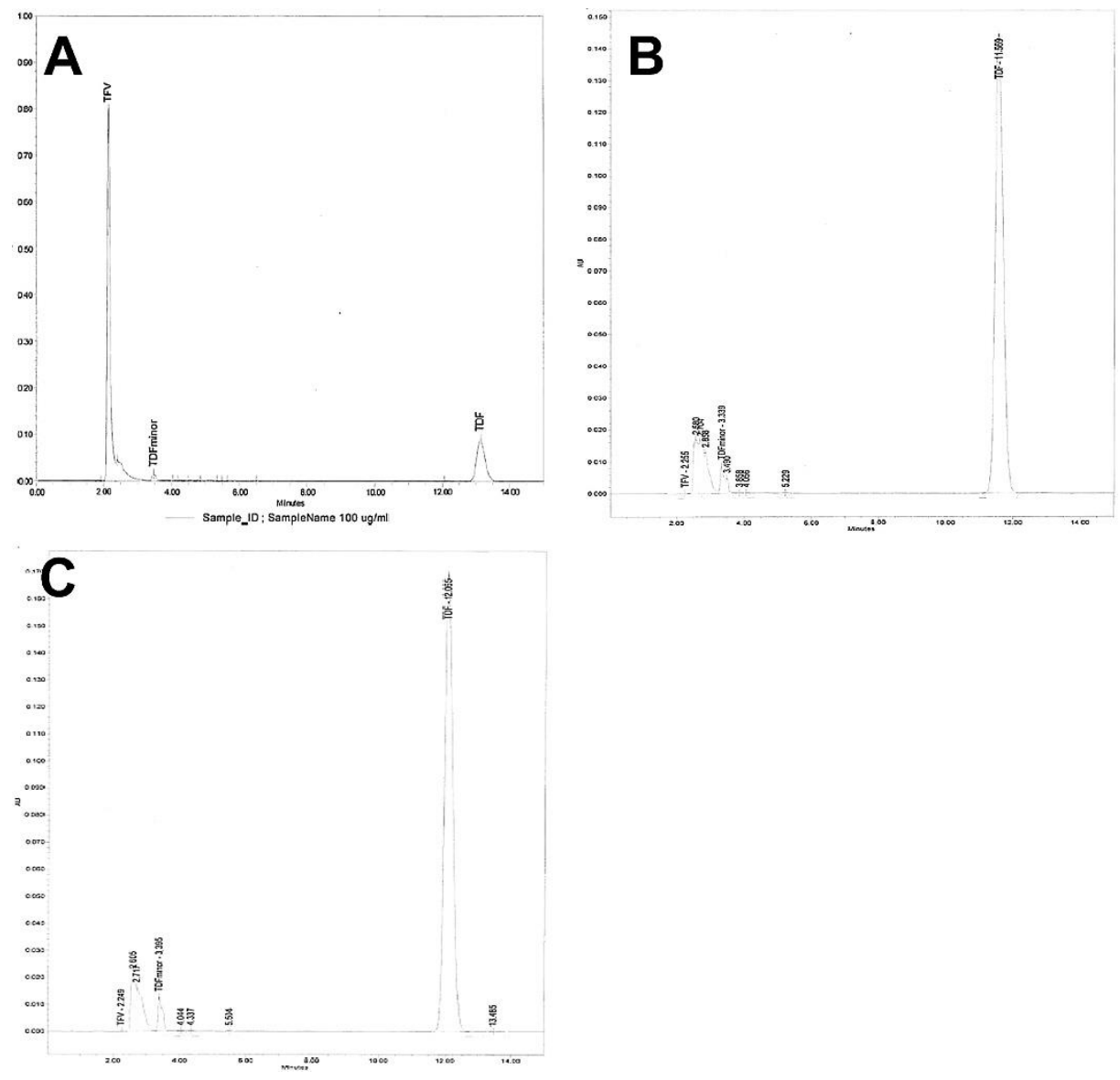

Supplemental Figure 2.1 HPLC chromatogram of $(\mathrm{A})$ a standard containing pure TDF and TFV, (B) 20\% PLGA TDF EF eluate, and (C) 20\% PLCL TDF EF eluate. (A) Standards containing both TFV and TDF were prepared to quantify TDF incorporated into fibers. TDF peaks elute at 12 min while TFV elutes 2 min after injection. For fiber samples, EFs were weighed and dissolved in DMSO and analyzed. (B) 20\% PLGA fibers and (C) 20\% PLCL fibers exhibited peaks for TDF while showing minimal hydrolysis products. The elution times were maintained throughout the experiment. 
Table 2.2 summarizes the total loading ( $\mu \mathrm{g}$ TDF/mg fiber) and encapsulation efficiency (EE) achieved for the various fiber formulations. Overall, we observed that fibers electrospun with HFIP resulted in high EEs spanning 60$89 \%$. Furthermore, proportional increases in loading were observed based on the amount of TDF added to PLGA and PLCL formulations. However, comparing polymer formulations electrospun with different solvents, PLGA and PLCL fibers electrospun with HFIP demonstrated higher loading and encapsulation efficiency, relative to PLGA and PLCL fibers electrospun with TFE. Ten percent TDF fibers electrospun with HFIP showed high encapsulation efficiencies spanning 76 to $89 \%$, relative to fibers electrospun with TFE ( 60\%). Considering the higher polymer concentration required to fabricate well-delineated fibers with TFE $(20 \%$ for PLGA and PLCL vs. 12 and 15\% for PLGA and PLCL, respectively) and the lower EE, HFIP was selected to electrospin subsequent formulations. 
Table 2.2 Quantification of TDF fiber loading and encapsulation efficiency. PLGA and PLCL fibers electrospun with HFIP demonstrated higher loading and encapsulation efficiency, relative to PLGA and PLCL fibers electrospun with TFE. Increases in encapsulation efficiency were observed based on the amount of TDF added to $10 \%$ TDF PLGA and PLCL formulations.

\begin{tabular}{|c|c|c|c|}
\hline \multicolumn{2}{|c|}{ Fiber Formulation } & $\begin{array}{c}\text { Loading TDF/Fiber } \\
(\mu \mathrm{g} / \mathrm{mg})\end{array}$ & $\begin{array}{c}\text { Encapsulation Efficiency } \\
(\%)\end{array}$ \\
\hline \multirow{3}{*}{ HFIP 15\% PLGA } & $1 \%$ TDF & $4.9 \pm 0.8$ & $80.7 \pm 12.4$ \\
\cline { 2 - 4 } & $10 \%$ TDF & $45.7 \pm 0.8$ & $81.4 \pm 1.5$ \\
\cline { 2 - 4 } & $\mathbf{2 0} \%$ TDF & $82.1 \pm 2.5$ & $76.3 \pm 2.3$ \\
\hline \multirow{3}{*}{ HFIP 12\% PLCL } & $1 \%$ TDF & $5.0 \pm 0.4$ & $81.1 \pm 5.8$ \\
\cline { 2 - 4 } & $10 \%$ TDF & $54.0 \pm 0.5$ & $89.7 \pm 0.8$ \\
\cline { 2 - 4 } & $\mathbf{2 0} \%$ TDF & $92.5 \pm 9.6$ & $80.4 \pm 8.3$ \\
\hline TFE 20\% PLGA $10 \%$ TDF & $52.3 \pm 3.5$ & $62.7 \pm 4.2$ \\
\hline \multicolumn{2}{|c|}{ TFE 20\% PLCL 10\% TDF } & $54.6 \pm 0.4$ & $60.4 \pm 0.4$ \\
\hline
\end{tabular}


After determining PLGA and PLCL fiber loading, we assessed the release of TDF from PLGA and PLCL EFs in SVF for up to 4 weeks (Figure 2.4). Figure 2.4A demonstrates increased TDF release per mass of fiber, and corresponds with increased incorporation of TDF in 1, 10, and 20\% PLGA and PLCL fibers. While both the 1\% TDF PLGA and PLCL formulations exhibited release near the limit of TDF detection, the cumulative release of TDF from the $10 \%$ and $20 \%$ PLCL fibers resulted in 16 and $26 \mu \mathrm{g} / \mathrm{mg}$ (31 and 29\% total release), while the PLGA fibers averaged only 9.3 and $19.7 \mu \mathrm{g} / \mathrm{mg}$ (20 and $22 \%$ total release) within $1 \mathrm{~h}$. Within the first $24 \mathrm{~h}$, the $10 \%$ and $20 \%$ formulations demonstrated a burst release, with the amount of TDF release increasing with increased TDF incorporation. Overall, the 10 and $20 \%$ PLGA fibers released up to $40 \%$ more TDF than PLCL fibers. Although appreciable increases in release were only observed for approximately $72 \mathrm{~h}$, PLGA fibers released more TDF than PLCL fibers for the 10 and 20\% TDF formulations. The 10\% PLGA and PLCL fibers released 66 and $39 \%$ of their cargo, respectively, while the 20\% PLGA and PLCL fibers released 64 and $43 \%$ of their cargo after 4 wk. 

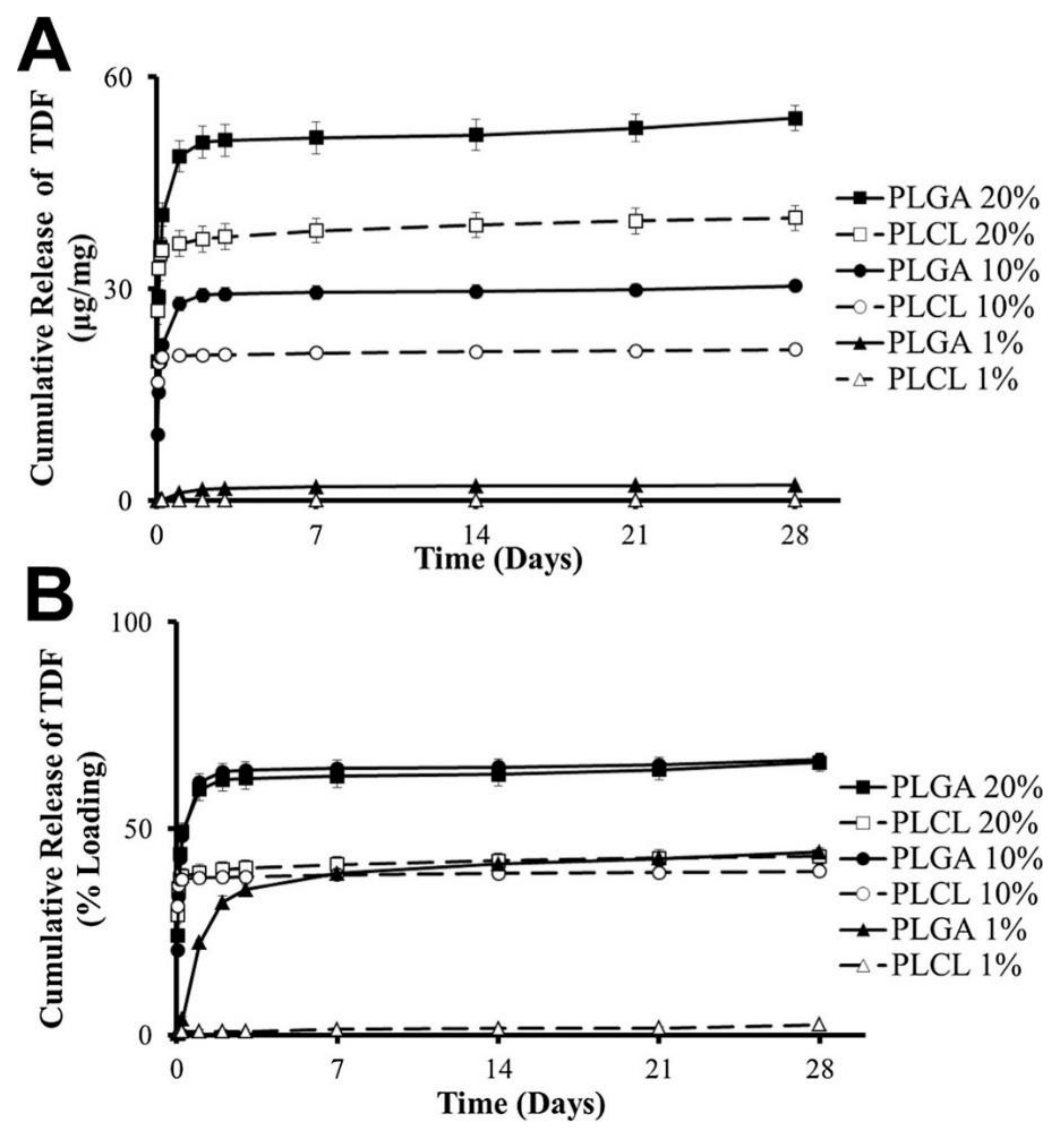

Figure 2.4 Release profiles of TDF from 1, 10, and 20\% TDF PLGA and PLCL fibers in SVF. (A) Cumulative release of TDF per milligram of fiber ( $\mu \mathrm{g}$ TDF/mg fiber) and (B) as percent total loading over 4 wk. While PLCL fibers showed a higher burst release after $1 \mathrm{~h}$, PLGA fibers exhibited greater release, as early as $24 \mathrm{~h}$, relative to PLCL fibers. 


\section{In Vitro Efficacy of PLGA and PLCL Fibers against HIV-1 Infection}

\section{Short-Term Efficacy}

To assess the antiviral activity of TDF PLGA and PLCL fibers, HIV-1 inhibition assays were performed using fiber eluates collected at different release time points. Both short- and long-term release samples were collected to assess efficacy. For short-term assessment of antiviral activity, $10 \mathrm{mg}$ fibers were incubated in $1 \mathrm{~mL}$ DMEM for 1 or $24 \mathrm{~h}$. A histogram of the HIV-1 inhibition after administration of the $1 \mathrm{~h}$ (Figure 2.5A) or $24 \mathrm{~h}$ fiber release eluates (Figure 2.5B) is shown, and the corresponding $\mathrm{IC}_{50}$ S are shown in Table 2.3. All 10 or $20 \%$ TDF fibers (PLGA or PLCL) completely inhibited viral infection in TZM-bl cells down to a 1:100 eluate dilution. For 1 and $24 \mathrm{~h}$ eluate dilutions exceeding 1:100, viral inhibition was more pronounced after administration of the $24 \mathrm{~h}$ eluates, relative to $1 \mathrm{~h}$ eluates. The increased efficacy observed with $24 \mathrm{~h}$ eluates can be attributed to the higher amount of TDF released within $24 \mathrm{~h}$. However, for both time points, the $\mathrm{IC}_{50 \mathrm{~S}}$ of both fibers were similar against in vitro infection (Table 2.3). 

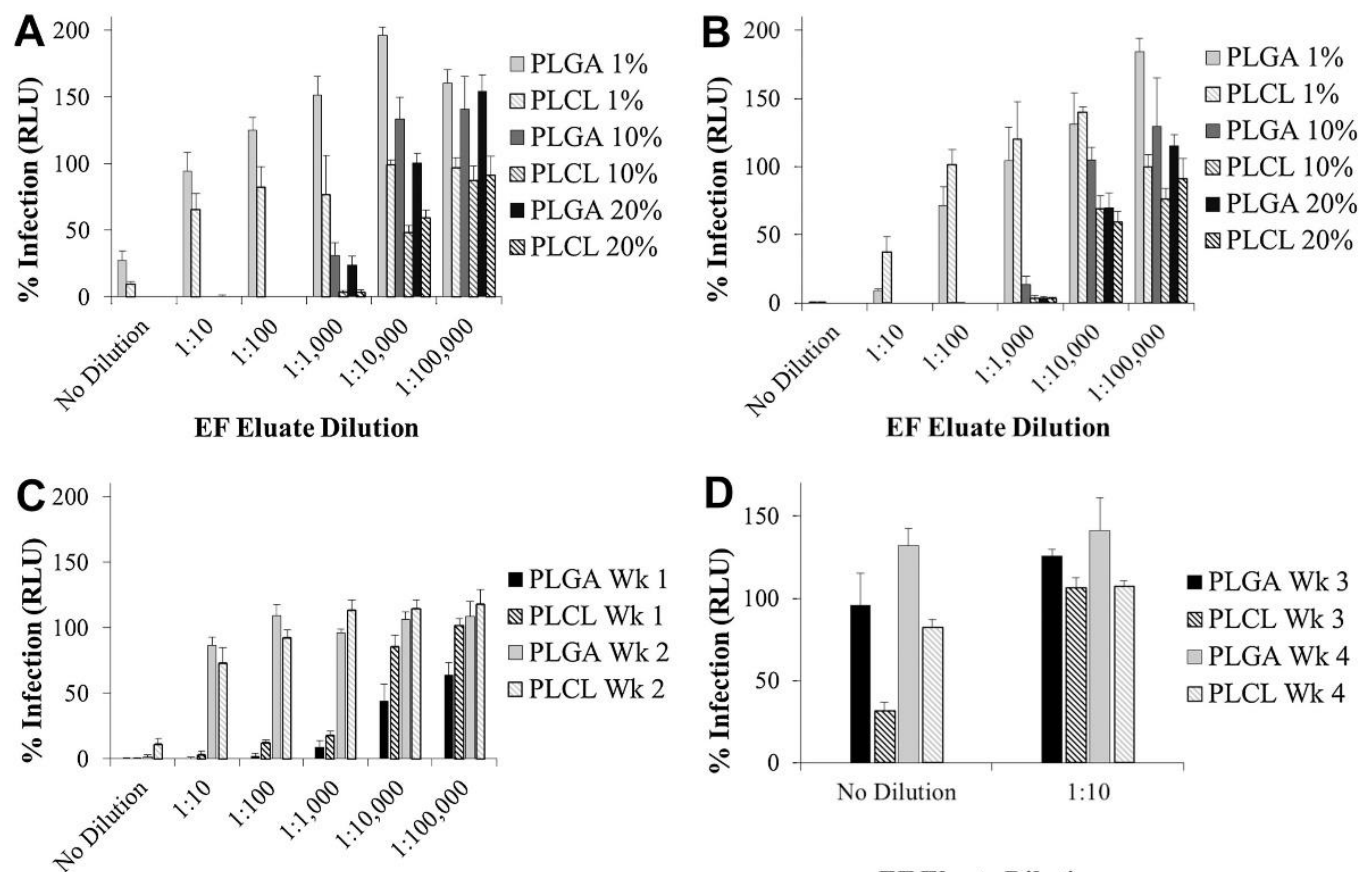

EF Eluate Dilution
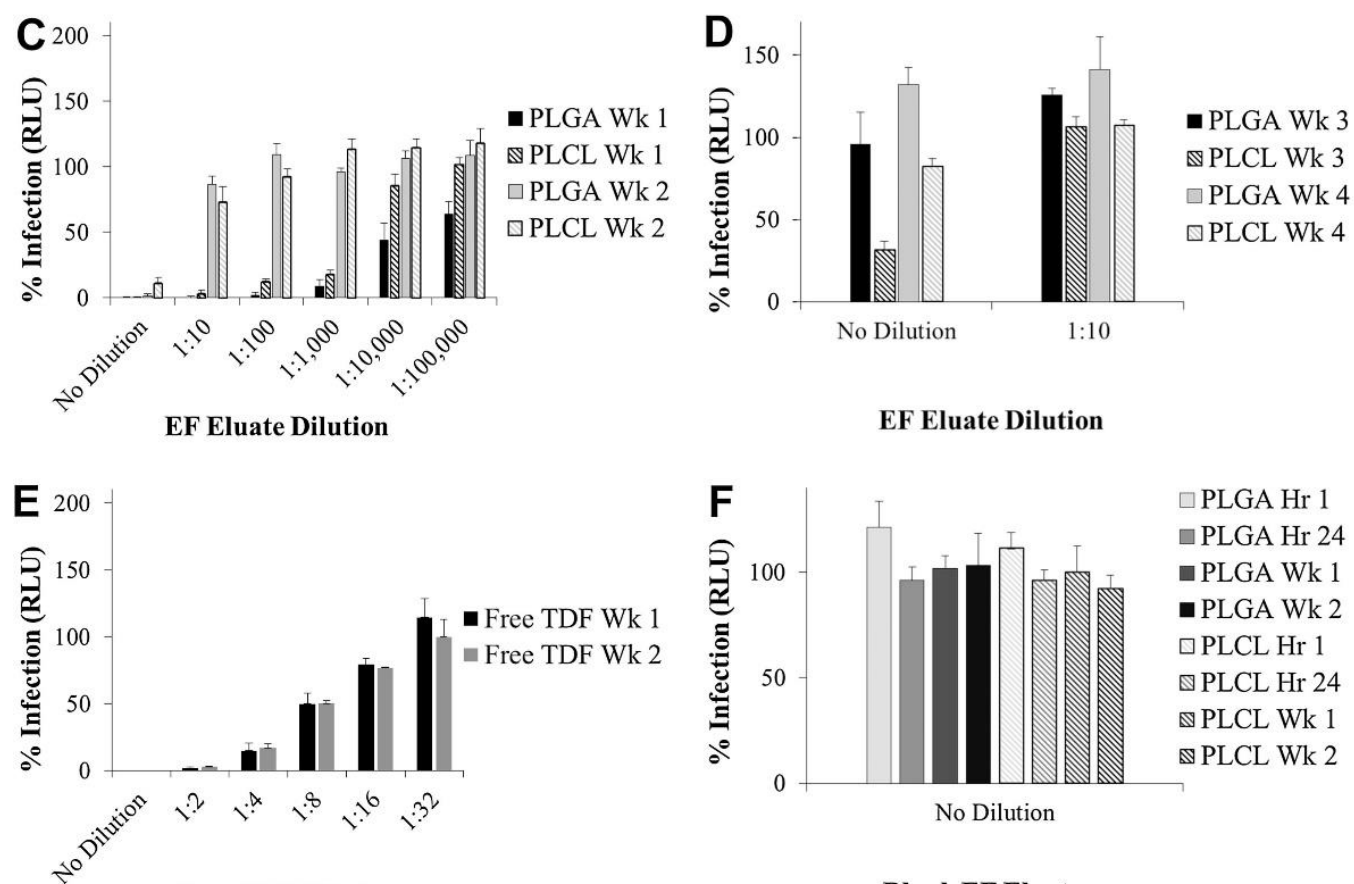

Free TDF Dilution

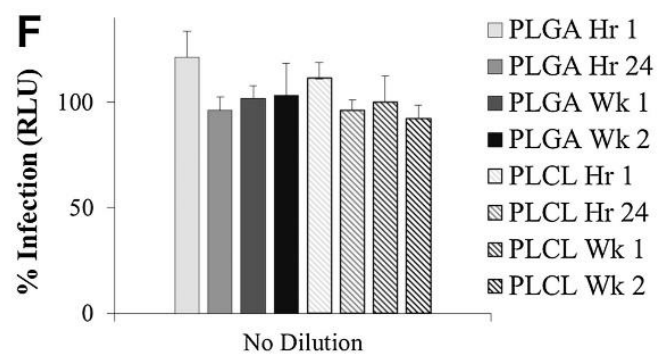

Blank EF Eluate

Figure 2.5 PLGA and PLCL fiber eluates inhibit HIV-1 infection in vitro after 1 and $24 \mathrm{~h}$, and 1 and 2 wk of release. Dilutions of release eluate from $10 \mathrm{mg} / \mathrm{mL}$ fiber concentrations at different time points were normalized to untreated/infected cell control RLUs to assess percent HIV-1 inhibition in vitro. Figures show the percent of cells infected, after incubation with: (A) 1 and (B) $24 \mathrm{~h}$ release eluates from all PLGA and PLCL EF formulations; (C) 1 and 2 wk release eluates from 20\% TDF PLGA and PLCL fibers; (D) 3 and 4 wk eluates from 20\% TDF PLGA and PLCL 
fibers; compared to (E) free TDF $(200 \mu \mathrm{g} / \mathrm{mL}) 1$ and 2 wk eluate, and (F) blank fiber eluates at each time point. 
Table 2.3 The IC 50 S of PLGA and PLCL fibers (against HIV-1) after administration of the 1 and $24 \mathrm{~h}$ release eluates. The IC 50 S of PLGA and PLCL TDF 1 and $24 \mathrm{~h}$ fiber eluates were similar to, or less than free TDF. Using one-way ANOVA with Bonferroni post hoc test, no statistical significance was observed between the $\mathrm{IC}_{50}$ S of fiber eluates taken at the same time, there was a statistically significant increase in the $\mathrm{IC}_{50}$ S of all formulations after $24 \mathrm{~h}$ release $(p<0.05)$. Confidence intervals of the $\mathrm{IC}_{50}$ s are shown in parentheses.

\begin{tabular}{|c|c|c|}
\hline Fiber Formulation & $\begin{array}{c}\mathbf{I C}_{50} \text { at } 1 \mathrm{Hr} \\
(\mathbf{n g} / \mathbf{m L})\end{array}$ & $\begin{array}{c}\mathbf{I C}_{50} \text { at } 24 \mathrm{Hr} \\
\text { (ng/mL) }\end{array}$ \\
\hline PLGA 10\% TDF & $2.4(2.0$ to 3.0$)$ & $7.2(6.0$ to 8.5$)$ \\
\hline PLGA 20\% TDF & $4.6(4.3$ to 5.0$)$ & $5.1(3.9$ to 6.7$)$ \\
\hline PLCL 10\% TDF & $3.1(2.5$ to 3.7$)$ & $7.4(4.2$ to 13.0$)$ \\
\hline PLCL 20\% TDF & $3.1(2.0$ to 4.7$)$ & $1.9(1.1$ to 3.5$)$ \\
\hline Free TDF & $12.1(10.9$ to 13.3$)$ & $5.3(3.4$ to 8.2$)$ \\
\hline
\end{tabular}


Overall, the antiviral activities of these eluate dilutions demonstrate that the amount of TDF in the fiber corresponds with increased HIV-1 inhibition. For the $1 \%$ TDF PLGA and PLCL fibers, $1 \mathrm{~h}$ undiluted eluates decreased infection to $27 \%$ and $10 \%$, relative to untreated/infected controls. However, subsequent dilutions of the 1\% TDF $1 \mathrm{~h}$ eluates yielded decreased protection against HIV-1 infection. In contrast, after $24 \mathrm{~h}$ release, the 1\% TDF PLGA and PLCL fibers completely inhibited virus infection, with subsequent decreases in virus inhibition corresponding with increased eluate dilution. Full infection resulted after administration of the 1\% PLGA and PLCL fibers at a dilution of 1:10 and 1:1000, respectively for the $1 \mathrm{~h}$ eluates; and 1:1000 and 1:100 for the PLGA and PLCL 24 h eluates.

For the 10\% TDF formulations, both PLGA and PLCL fibers exhibited complete protection against HIV-1 down to $1: 100$ and 1:1000 eluate dilutions, respectively after $1 \mathrm{~h}$. After administration of the $24 \mathrm{~h}$ eluates, complete protection was observed even after a 1:1000 dilution of each formulation. Subsequent dilutions of PLGA eluates for $1 \mathrm{~h}$ and $24 \mathrm{~h}$ time points exhibited an increase in infectivity (decrease in prevention) to $31 \%$ and $13 \%$ for $1: 1000$, and complete infectivity for 1:10,000 dilutions, respectively. For PLCL, complete protection was achieved with the 1:1000 eluate dilutions at both 1 and $24 \mathrm{~h}$ time points. Additionally, these fibers showed efficacy even at eluate dilutions of 1:10,000; with $48 \%$ and $69 \%$ infectivity at 1 and $24 \mathrm{~h}$. The corresponding $\mathrm{IC}_{50}$ s for $10 \%$ TDF PLGA and PLCL EFs were 2.4 and $3.1 \mathrm{ng} / \mathrm{mL}$ TDF after $1 \mathrm{~h}$, and 7.2 and $7.4 \mathrm{ng} / \mathrm{mL}$ after $24 \mathrm{~h}$ (Table 2.3). 
As expected, the $20 \%$ TDF fibers exhibited the highest efficacy against HIV1 infection per mass of fiber tested. Similar to the 10\% TDF PLGA and PLCL EFs, $20 \%$ TDF fibers completely inhibited infection after a 1:1000 dilution, and exhibited partial efficacy (58-100\%) between 1:10,000 and 1:100,000 dilutions. The corresponding $\mathrm{IC}_{50}$ s for 20\% TDF PLGA and PLCL EFs were 4.6 and $3.1 \mathrm{ng} / \mathrm{mL}$ after $1 \mathrm{~h}$ release, and 5.1 and $1.9 \mathrm{ng} / \mathrm{mL}$ after $24 \mathrm{~h}$ release (Table 2.3). Despite these small differences, the PLGA and PLCL TDF fibers were equally efficacious at their respective time points (Table 2.3). While statistical analysis was performed between each formulation and time point, no clear pattern emerged. There was no statistical significance between any of the formulations after $1 \mathrm{~h}$ release, with the exception of free TDF, which had a significantly higher $\mathrm{IC}_{50}$ than the TDF fiber formulations. However, after $24 \mathrm{~h}$ there was marked decrease of the $\mathrm{IC}_{50}$ of free TDF (12.1 and $5.3 \mathrm{ng} / \mathrm{mL}$ at 1 and $24 \mathrm{~h}$ respectively) which resulted in no statistical difference between free TDF and fibers. However, most of the $\mathrm{IC}_{50 \mathrm{~S}}$ at $24 \mathrm{~h}$ possessed statistical significant differences at that time point. Furthermore, the $\mathrm{IC}_{50 \mathrm{~S}}$ at $24 \mathrm{~h}$ generally were larger than their $1 \mathrm{~h}$ counterparts, although this trend was not seen in all formulations. Considering that all EF formulations possessed similar IC 50 to their free TDF counterparts, suggests that any formulation could be used to provide short-term protection for 1 or $24 \mathrm{~h}$.

\section{Long-Term Efficacy}

To assess the long-term efficacy of the fibers against HIV-1 infection in vitro, eluates were collected from $10 \mathrm{mg}$ of $20 \%$ TDF PLGA and PLCL fibers after 1, 2, 3, and 4 wk. Twenty percent TDF fibers were selected due to their greater 
encapsulation, release, and applicability to future dosing in vivo. The resulting HIV1 inhibition after administration of 1 and 2 wk fiber release eluates is shown in Figure 2.5C and the corresponding $\mathrm{IC}_{50} \mathrm{~S}$ are quantified in Table 2.4. After 1 and 2 wk, eluates from $10 \mathrm{mg} / \mathrm{mL}$ PLGA and PLCL EFs completely inhibited HIV-1 infection. However, only the 1 wk eluates completely inhibited HIV-1 infection, after a 1:100 or 1:1000 dilution. Two week eluates demonstrated weaker activity, showing only marginal protection (14\% and $27 \%$ ) at 1:10 dilutions of PLGA and PLCL fiber eluates, respectively. The corresponding $\mathrm{IC}_{50}$ S were 1.9 and 11.9 $\mathrm{ng} / \mathrm{mL}$ for 1 wk PLGA and PLCL eluates and 10.2 and $72.3 \mu \mathrm{g} / \mathrm{mL}$ for 2 wk PLGA and PLCL eluates. Free TDF controls showed a similar decrease in inhibition, relative to their $\mathrm{IC}_{50}$ S after 1 and $24 \mathrm{~h}$ exposure to media (12 and $5.2 \mathrm{ng} / \mathrm{mL}$ ), to 4.5 and $4.9 \mu \mathrm{g} / \mathrm{mL}$ at 1 and $2 \mathrm{wk}$, suggesting the hydrolysis of free TDF into its monoester derivative after prolonged exposure to media (Figure 2.5E, which has been documented in similar studies ${ }^{56}$. Undiluted eluates from weeks 3 and 4 showed minimal protection against HIV-1 at the doses tested (Figure 2.5D). Blank fiber eluates were tested as a negative control for inhibition (Figure 2.5F). The decreased efficacy of TDF EFs against HIV-1 may be attributed to increased levels of mPTFV within the solution, as well as decreased TDF release from the fiber. 
Table 2.4 The $\mathrm{IC}_{50 \text { s }}$ of PLGA and PLCL fibers (against HIV-1) after administration of 1 and 2 wk release eluates. As exposure time to media increased, the efficacy of TDF fibers decreased. However, both PLGA and PLCL fiber eluates were more efficacious than free TDF after $1 \mathrm{wk}$ exposure to media. Confidence intervals of the $\mathrm{IC}_{50} \mathrm{~S}$ are shown in parentheses.

\begin{tabular}{|c|c|}
\hline Fiber Formulation & IC $_{\mathbf{5 0}}$ (ng/mL) \\
\hline PLGA 20\% TDF (1 Wk) & $1.9(0.9$ to 4.1$)$ \\
\hline PLGA 20\% TDF (2 Wk) & $10,181(3,631$ to 25,550$)$ \\
\hline PLCL 20\% TDF (1 Wk) & $11.9(9.0$ to 15.9$)$ \\
\hline PLCL 20\% TDF (2 Wk) & $7,226(3,947$ to 13,229$)$ \\
\hline Free TDF (1 Wk) & $4,504(3,858$ to 5,259$)$ \\
\hline Free TDF (2 Wk) & $4,927(4,470$ to 5,432$)$ \\
\hline
\end{tabular}


As the incubation time increased, the level of protection seen from both TDF EFs and free TDF decreased. Although 1 wk PLGA fiber eluates showed higher efficacy $(1.9 \mathrm{ng} / \mathrm{mL})$, relative to PLCL $(11.9 \mathrm{ng} / \mathrm{mL})$, the $\mathrm{IC}_{50}$ s were not statistically significant. However, when compared to free TDF, the $\mathrm{IC}_{50}$ s of both PLGA and PLCL fibers demonstrated statistically significant increases in protection $(p<0.05$, Table 2.4). Furthermore, all $I C_{50 S}$ of the 1 wk eluates were statistically significant $(p<0.05)$ relative to the $1 \mathrm{~h}, 24 \mathrm{~h}$, and 2 wk time points. Thus for applications spanning one week, TDF fibers demonstrated prolonged activity, relative to free TDF. Additionally, less PLGA fiber was needed, relative to PLCL, to release therapeutically relevant amounts of TDF.

\section{In Vitro Efficacy of PLGA and PLCL Fibers against HSV-2 Infection}

To evaluate the potential of these fibers to inhibit HSV-2 infection, the antiviral efficacy of the $20 \%$ TDF EFs was also assessed in HSV-2 plaque assays. Similar to the HIV-1 infection assay, eluate from $5 \mathrm{mg} / \mathrm{mL}$ fibers at 1 and $24 \mathrm{~h}$ was shown to completely inhibit viral plaque formation. Figure 2.6 illustrates the results of 1 and $24 \mathrm{~h}$ eluate serial dilutions on infectivity. Both PLGA and PLCL fiber

eluates completely inhibited HSV-2 infection, and exhibited decreased protection with increased dilution. Eluates from $24 \mathrm{~h}$ showed greater efficacy against HSV-2 infection due to the increased amount of released TDF. 

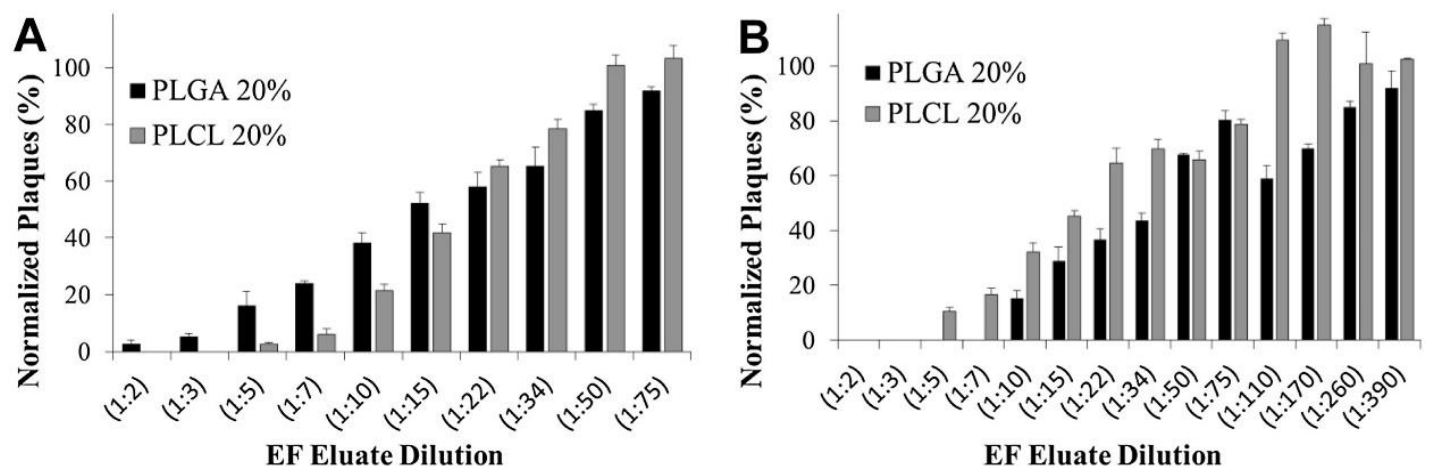

Figure 2.6 Both PLGA and PLCL fiber release eluates prevent HSV-2 infection in vitro. Plaque assays were conducted to assess the efficacy of 20\% TDF PLGA and PLCL fiber eluates $(5 \mathrm{mg} / \mathrm{mL})$ against HSV-2 infection in vitro. Plaques were counted and normalized to untreated/uninfected cells. Results demonstrate HSV2 efficacy attained with: (A) 1 and (B) $24 \mathrm{~h}$ fiber eluate dilutions. 
The IC50s of PLGA and PLCL TDF fibers were assessed using these eluate dilutions. Table 2.5 shows that the IC50S of PLGA and PLCL 1 and $24 \mathrm{~h}$ eluates were comparable to the $\mathrm{IC}_{50}$ of free TDF $(8.9 \mu \mathrm{g} / \mathrm{mL})$. The plaque assays showed a trend of enhanced protection across dilutions provided by PLGA EFs after 1 and $24 \mathrm{~h}$, compared with PLCL fibers. However, no statistical significance was observed between formulations or compared to free TDF. Fiber eluates were also collected to assess HSV-2 protection after 1, 2, and 3 wk. For these time points and fiber concentration $(5 \mathrm{mg} / \mathrm{mL})$ tested, no virus inhibition was observed (data not shown). Similar to the HIV-1 studies, both $20 \%$ EF formulations demonstrate similar short-term protection compared with free TDF. 
Table 2.5 The IC50S of PLGA and PLCL fibers (against HSV-2) after administration of the 1 and $24 \mathrm{hr}$ release eluates. Plaque assays were performed to assess the antiviral activity of $20 \%$ TDF fiber eluates against HSV-2 infection. Fiber eluates from 1 and $24 \mathrm{hr}$ showed similar activity, relative to free TDF. Confidence intervals of the $\mathrm{IC}_{50}$ are shown in parentheses.

\begin{tabular}{|c|c|}
\hline Fiber Formulation & IC $_{50}(\mu \mathrm{g} / \mathrm{mL})$ \\
\hline PLGA 20\% TDF (1 hr) & $7.3(6.6$ to 8.1$)$ \\
\hline PLGA 20\% TDF (24 hr) & $14.3(13.4$ to 15.3$)$ \\
\hline PLCL 20\% TDF (1 hr) & $14.0(13.5$ to 14.5$)$ \\
\hline PLCL 20\% TDF (24 hr) & $20.3(18.3$ to 22.5$)$ \\
\hline Free TDF & $8.9(4.2$ to 18.8$)$ \\
\hline
\end{tabular}




\section{In Vitro and EpiVaginal Cytotoxicity of PLGA and PLCL Fibers}

To assess the potential of these fibers to safely interact with epithelial cells, fiber cytotoxicity was assessed in VK2, Ect1, and End1 E6E7 cells, using the MTT assay. As seen in Figure 2.7, high cell viability was maintained after administration of TDF fibers for 24,48 , and $72 \mathrm{~h}$. All cells demonstrated greater than 93,91 , and $96 \%$ cell viability at 24,48 , and $72 \mathrm{~h}$ respectively, for all formulations tested. In addition to cell monolayers, EpiVaginal tissue viability after PLGA fiber application was examined using the LDH cytotoxicity assay and H\&E staining (Figure 2.8). Due to the limited availability of EpiVaginal tissue samples, PLGA EFs were selected for analysis due to their enhanced release profiles and therapeutic potential, relative to PLCL fibers. PLGA EFs incorporating 20\% TDF (5 and 50 $\mathrm{mg} / \mathrm{mL}$ ) were incubated 48 and $72 \mathrm{~h}$ with EpiVaginal tissue. Microscopic examination of the untreated, blank PLGA EF-treated, and TDF PLGA EF-treated tissues, revealed normal full thickness vaginal epithelium, while tissue treated with $0.2 \%$ non-oxynol $9(\mathrm{~N}-9)$ exhibited severe degeneration of the epithelium (Figure 2.8A). No adverse histological findings were noted in EpiVaginal tissue treated with PLGA or TDF PLGA EFs. Furthermore, tissue incubated with either 5 or 50 $\mathrm{mg} / \mathrm{mL}$ fiber exhibited comparable viability in the LDH assay relative to untreated tissue controls after 48 and $72 \mathrm{~h}$ (Figure 2.8B). 
A
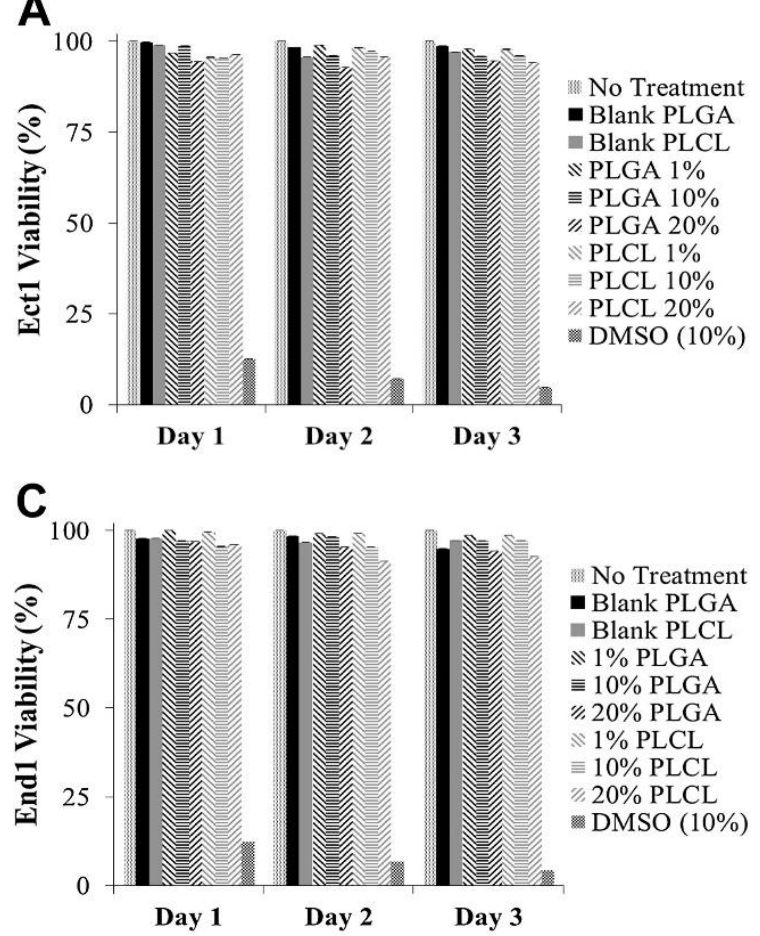

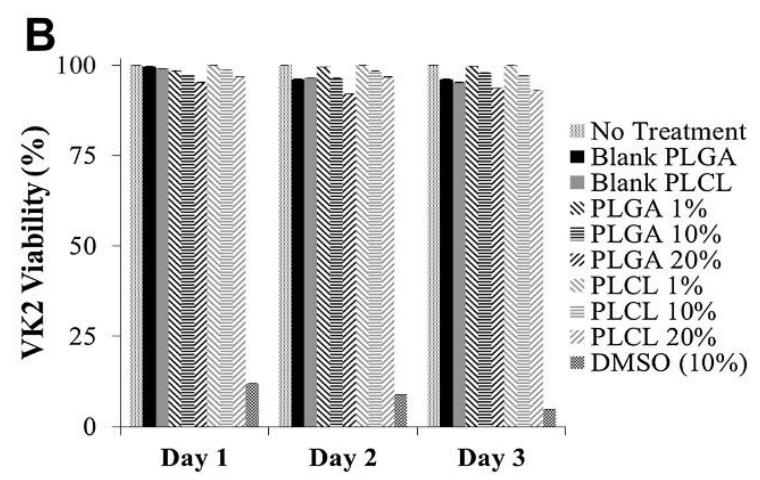

Figure 2.7 Cytotoxicity assessed via MTT assay. Vaginal epithelial cell lines: (A) Ect1 E6/E7, (B) VK2 E6/E7, (C) and End1 E6/E7, were incubated with blank or $20 \%$ TDF PLGA or PLCL fibers $(10 \mathrm{mg} / \mathrm{mL})$ for 24,48 , and $72 \mathrm{~h}$. All cells demonstrated greater than 93,91 , and $96 \%$ cell viability at 24,48 , and $72 \mathrm{~h}$ respectively, for all formulations tested. 
A

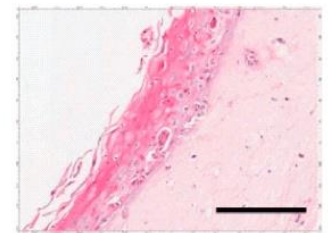

Untreated

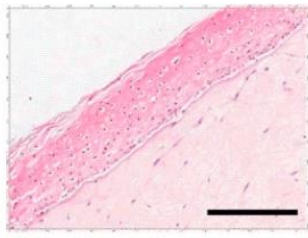

TDF PLGA EF

(5 $\mathrm{mg} / \mathrm{mL})$

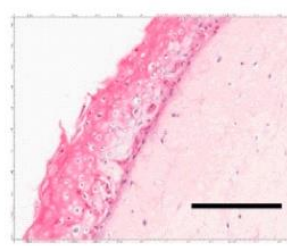

Blank PLGA EF

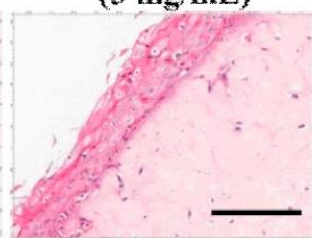

TDF PLGA EF

(50 $\mathrm{mg} / \mathrm{mL}$ )

$0.2 \% N-9$

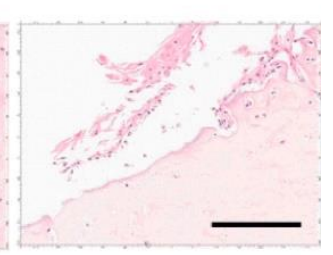

$0,2 \% \mathbb{N}-9$

B

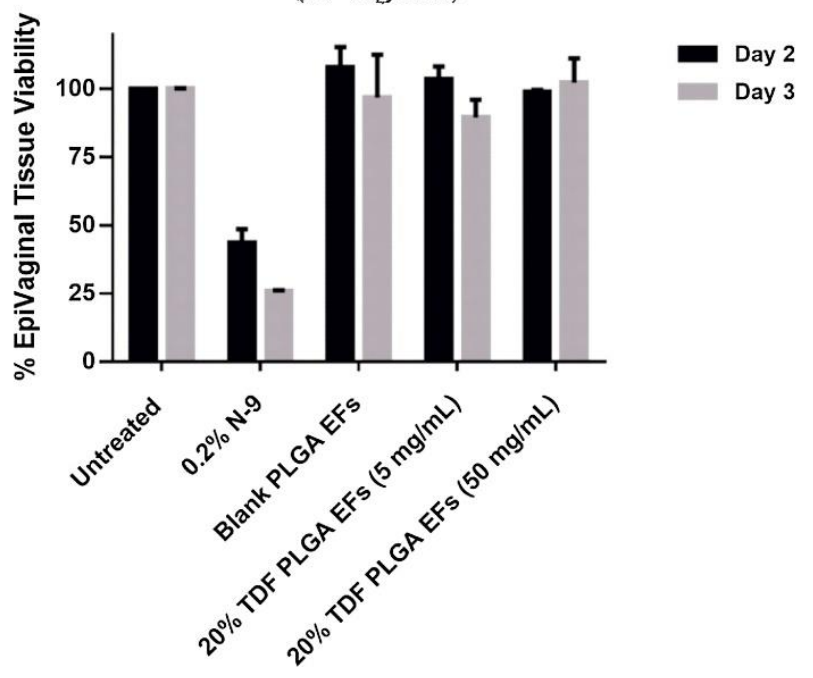

Figure 2.8 EpiVaginal cytotoxicity was assessed via H\&E staining (A) and LDH assay (B). (A) H\&E stained cross-sections of EpiVaginal VEC-100-FT tissues following 3-day exposure to PLGA TDF fibers, relative to untreated and toxic control $(0.2 \% \mathrm{~N}-9)$ groups. Scale bar represents $200 \mu \mathrm{m}$. (B) Tissue viability (LDH) measurements for EpiVaginal VEC-100-FT tissues following two or three day exposure to PLGA TDF fibers, relative to untreated and toxic control $(0.2 \% \mathrm{~N}-9)$ groups. 
In parallel, cytokine production from EpiVaginal tissue was analyzed after 48 and $72 \mathrm{~h}$ of PLGA fiber administration. Cytokine expression, including GM-CSF, IFN- $\gamma$, IL-1 $\alpha, I L-1 \beta, I L-6, I L-8$, MCP-1 and TNF- $\alpha$, was compared to untreated and N-9 treated controls (Figure 2.9). After $48 \mathrm{~h}$ administration of TDF EFs, only GMCSF and IL-6 expression showed statically significant increase in expression (approximately two-fold) compared with untreated tissue. After $72 \mathrm{~h}$, only the 50 $\mathrm{mg} / \mathrm{mL}$ TDF fibers showed a slight increase of GM-CSF and MCP-1 cytokine expression (1.3-fold increase for both) relative to untreated tissue. Cytokine expression from tissue samples exposed to blank fibers was comparable with untreated samples at both time points, showing no statistical significance. In contrast, EpiVaginal tissue exposed to $\mathrm{N}-9$ for $48 \mathrm{~h}$ showed a marked decrease in GM-CSF, IFN- $\gamma$, IL-6, MCP-1, and TNF- $\alpha(0.7,0.1,0.12$, and 0.4 respectively) while showing a slight increase of both $\mathrm{IL}-1 \alpha$ and $\beta$ (1.7 and 1.2-fold increases, respectively). After $72 \mathrm{~h}$, the expression of all cytokines following N-9 treatment was lower than observed in untreated samples, which is attributed to the loss of the vaginal epithelium. The negligible increase in cytokine expression (0-2 fold difference) from exposure of TDF EFs demonstrates promising preliminary safety profiles of these fiber formulations ${ }^{69}$. 


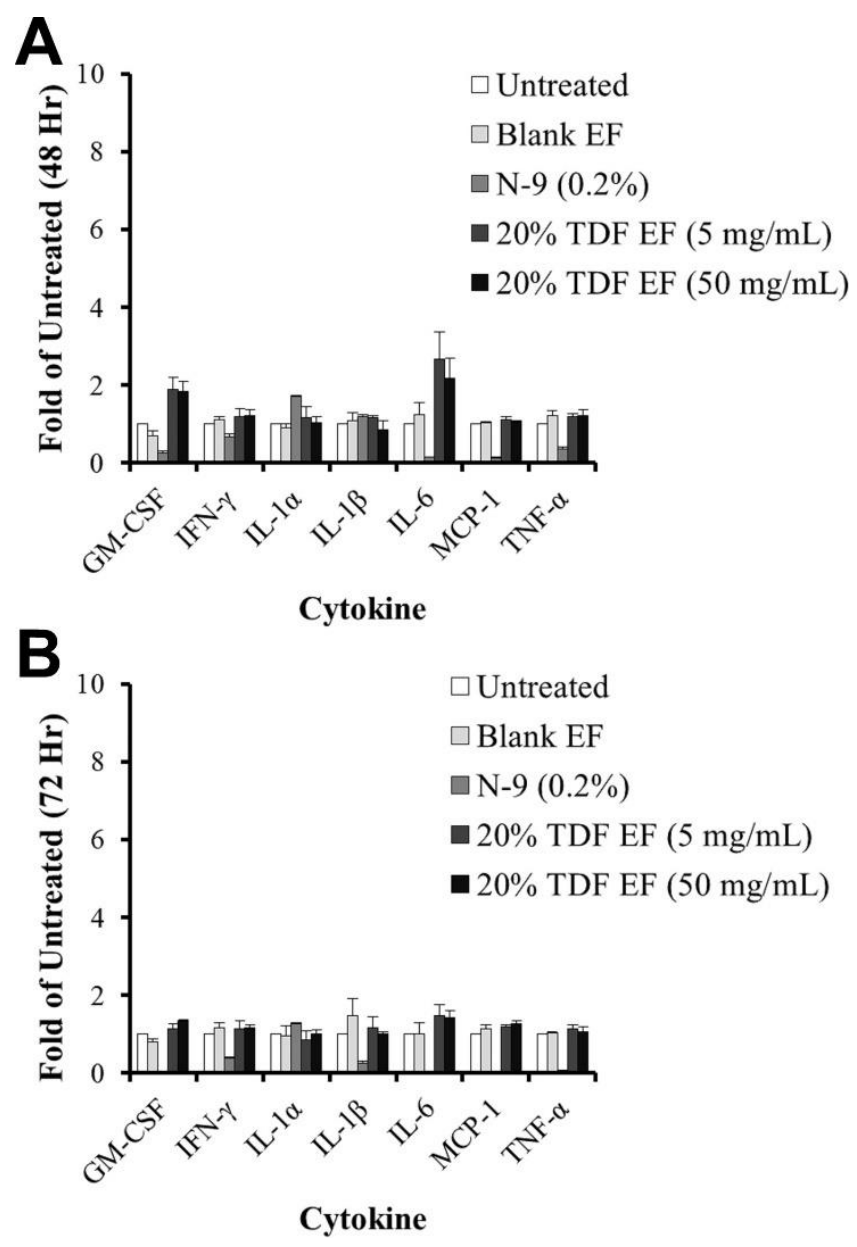

Figure 2.9 Cytokine expression from EpiVaginal studies after (A) 48 and (B) $72 \mathrm{~h}$ administration of fibers. TDF EFs elicited minimal cytokine expression relative to untreated samples after $72 \mathrm{~h}$. Positive $\mathrm{N}-9$ treated samples failed to induce cytokine expression due to epithelial cell death. 


\section{Discussion}

There is an urgent need for new topical PrEP technologies that can confer the sustained-release of active agents, while providing discreet and convenient protection against STIs. The emerging application of polymeric electrospun fibers for intravaginal delivery offers the potential to fill this unique role. In these studies, we evaluated two electrospun fiber delivery platforms, comprised of PLGA or PLCL polymers, for their ability to release TDF, and protect against both HIV-1 and HSV2 infections in vitro. Here TDF served as a model antiretroviral drug, as it is only one of two agents approved by the FDA to prevent HIV-1 infection. Furthermore, TDF has demonstrated antiviral activity against both HIV-1 and HSV-2 in vivo, establishing its versatility as a multipurpose active agent. The goal of this work was to develop and characterize polymeric electrospun fibers to safely and efficaciously provide protection against both HIV-1 and HSV-2 in vitro, as a potential multipurpose prevention platform. For the doses tested in our studies, TDF PLGA and PLCL fibers demonstrated equivalent protection, relative to free TDF, against both HIV-1 and HSV-2 infections upon exposure to short-term (24 h) release eluates. In addition, enhanced efficacy of TDF EFs compared with free TDF against HIV-1 was demonstrated after exposure to release eluates taken through 2 wk. Moreover, this is the first time the safety of PLGA and PLCL TDF fibers has been investigated in EpiVaginal tissue.

The first goal of this study was to determine the formulation of TDF PLGA and PLCL fibers that resulted in the most cohesive and well-defined fiber macroand microstructures. During the fabrication of blank PLGA and PLCL fibers, 
several solvents were assessed (Figure 2.1). We observed that both HFIP and TFE solvents yielded reproducible PLGA and PLCL fiber morphologies. These solvents also enabled the incorporation of high weight percent polymer to solvent, which is critical to incorporating high concentrations of active agents in polymers. Using PLGA and PLCL fibers electrospun with HFIP as our baseline platforms, we sought to evaluate the effect of TDF incorporation on fiber diameter (Figure 2.2, Figure 2.3).

Fiber diameter has a critical role in the release properties of active agents. Previous research has shown that decreasing fiber diameter can enhance the release of active agents. This is attributed to the increased surface-to-volume ratio, and decreased distance necessary for encapsulate diffusion ${ }^{253,254}$. In our studies, the incorporation of TDF resulted in decreased fiber diameters relative to blank fibers (Figure 2.3). The diameters of blank fibers ranged from 1.7 to $2.0 \mu \mathrm{m}$; whereas TDF fiber diameters ranged from 0.7 to $1.1 \mu \mathrm{m}$. Even for the lowest concentration (1\%) TDF fibers tested, a $50 \%$ decrease in fiber diameter was observed. This decrease in fiber diameter may be attributed to the charge of the active agent, and/or increased polymer jet instability resulting from these charge effects. Correspondingly, this jet instability may result in the polymer traveling longer distance/duration during the electrospinning process prior to reaching the mandrel, promoting elongation and decreased fiber diameter 255,256 . Thus, incorporated active agents can affect the microstructural morphologies and diameters of electrospun fibers. 
The incorporation of antiviral or biological agents has been shown to affect fiber diameter in a number of ways. In previous studies, Tenofovir (TFV), a compound less hydrophobic (solubility $=1.87 \mathrm{mg} / \mathrm{mL}$ ) than TDF (the phosphorylated fumaric salt form of TFV, used in this study $0.71 \mathrm{mg} / \mathrm{mL}^{257,258}$, was shown to slightly increase fiber diameter, though the differences were not statistically significant ${ }^{166}$. In other studies, the incorporation of antivirals resulted in the opposite effect on fiber diameter. Incorporation of TFV in polyvinyl alcohol polymers resulted in slightly smaller diameters, attributed to the increased instability described above ${ }^{242}$. Finally, some experiments show no change in fiber diameter after active agent incorporation. Fibers comprised of the $\mathrm{pH}$-responsive CAP polymer, incorporating TDF, showed no change in fiber diameter relative to blank fibers ${ }^{83}$. These studies highlight that a variety of parameters including solvent choice, polymer selection, solvent-polymer/polymer-drug interactions, active agent characteristics, and solvent viscosity all contribute to the microstructural properties of electrospun fibers.

After obtaining well-defined and reproducible EFs, we next assessed the loading of TDF in PLGA and PLCL fibers as a function of solvent type (HFIP vs. TFE) used in the electrospinning process. From these loading studies (Table 2.2), we observed that fibers electrospun with HFIP showed $\sim 30 \%$ higher encapsulation efficiency, relative to fibers electrospun with TFE. Furthermore, higher polymer concentrations were needed to obtain well-defined fiber microstructure, based on solvent type (15 and 12\% w/w for PLGA and PLCL in HFIP; 20\% PLGA and PLCL in TFE). Despite both HFIP and TFE sharing many characteristics such as high 
polarity and similar molecular structure, there are several key differences that may impact fiber properties. As previously described ${ }^{87}$, TFE has a higher dielectric constant $(26.1 \mathrm{~F} / \mathrm{m})$ compared to HFIP $(16.7 \mathrm{~F} / \mathrm{m})^{259}$. This increased charge capacity may confer additional instability to TFE solvents during electrospinning, requiring more polymer to produce well-defined fiber morphology ${ }^{260}$. Additionally, this increase in dielectric constant may result in decreased TDF incorporation, and even TDF localization on or near the fiber surface. HFIP also possesses a much lower boiling point $\left(58.2^{\circ} \mathrm{C}\right)$ relative to TFE $\left(73.6^{\circ} \mathrm{C}\right)^{261,262}$. Solvents with lower boiling points tend to produce more stable fiber morphology due to complete evaporation during electrospinning; whereas less volatile (higher boiling point) solvents may not fully evaporate from the polymer, causing beaded morphologies ${ }^{76}$. These undesirable properties, in addition to the higher polymer concentrations required to produce TFE fibers with well-defined fiber microstructures and lower loading efficiencies, prompted us to fabricate subsequent formulations with HFIP.

Controlled release studies using TDF EFs yielded several interesting results (Figure 2.4). First, as expected with most polymeric delivery vehicles ${ }^{263}$, PLGA and PLCL formulations demonstrated a burst release of TDF during the first $24 \mathrm{~h}$. The exception was 1\% TDF fibers, which released TDF quantities near our limit of detection. While the $10 \%$ and $20 \%$ TDF PLCL fibers showed a higher burst release relative to 10 and $20 \%$ PLGA fibers within the first hour, after $24 \mathrm{~h}$, all PLGA formulations released more TDF. While burst release is a common phenomenon in polymer drug delivery ${ }^{263}$, here TDF surface localization may be exacerbated 
during the electrospinning process, due to charge effects between the incorporated drug, polymers, and solvent. Solution instability during electrospinning due to these charge effects, as well as hydrophilic interactions between the solvent and drug can also result in agent localization near the fiber surface 256,264 . In the case of PLCL EFs, due to the increased hydrophobicity, more TDF may have accumulated on the fiber surface, increasing burst release within the first $1 \mathrm{~h}$. Another observation in the release studies was that all PLGA fibers released higher percentages of TDF compared to PLCL fibers, after $\sim 1 \mathrm{wk}$ in SVF. We attribute this increased TDF release to the hydrophilicity of PLGA, enabling enhanced wettability of the fiber, resulting in increased diffusion of TDF from the fiber into the surrounding eluate 259,265 .

Other studies using similar polymers yielded similar release results. In one recent study, TFV (relative to TDF) was incorporated in PLGA and PCL polymers and polymer blends, and controlled release was evaluated for 10 days ${ }^{85}$. Similar to our work, PLGA demonstrated greater overall release of drug while showing an initial lower burst release. In contrast, PCL released all incorporated TFV after 24 h, while PLGA released only $\sim 20 \%$ of incorporated drug during this time. While TDF was not extensively evaluated, similar burst release of TDF was also observed with 20:80 PCL:PLGA fibers, a trend that differed from the prolonged release observed from polymer blends that incorporated TFV 266 . These studies highlighted the effect that small molecular changes in drug design can have on the release kinetics from polymeric delivery vehicles. Furthermore, several formulations of PCL/PLGA blends were fabricated, demonstrating decreased burst 
release of TFV with increasing PLGA concentration ${ }^{29}$. We expect that in future work, similar blends will prove favorable to tailor the release of TDF, despite its increased lipophilicity.

Polymer hydrophobicity is another important consideration for providing sustained-release. In another study relevant to microbicide delivery, the antiviral compounds, MVC (Maraviroc) and AZT, were encapsulated in PCL, polyethylene oxide (PEO), and poly-I-lactic acid (PLLA) polymer blends. Sustained-release from 70:30 PEO/PLLA blends showed almost complete release of hydrophilic compounds after $1 \mathrm{~h}$, due to the hydrophilicity and quick degradation of PEO. In contrast, 30:70 PEO:PLLA blends exhibited lower burst release and higher sustained-release relative to the more hydrophilic 70:30 PEO:PLLA blends. Additionally, the moderately hydrophobic 30:70 PEO:PLLA fibers demonstrated better release profiles relative to pure PCL fibers, which released around $95 \%$ of incorporated drugs after $1 \mathrm{~h}$. This more efficacious release profile was attributed to the intermediate hydrophobicity and crystallinity of PLLA compared to PEO and $\mathrm{PCL}^{86}$. Whereas the hydrophilicity of PEO confers quick degradation in aqueous solutions, resulting in burst release; PCL is highly hydrophobic, causing incorporated compounds to localize on the fiber surface, thereby significantly contributing to high burst release. The results from these studies are in agreement with our observations that polymers comprised of lactic and glycolic acid, relative to the more hydrophobic PLCL (or PCL), exhibit less burst release of moderately hydrophilic compounds. Moreover they emphasize the advantages of fabricating blended formulation to tune release properties. 
After characterizing these fibers, TDF EFs were evaluated for their potential to protect against HIV-1 and HSV-2 infections in vitro (Figure 2.5, Figure 2.6). In these studies, TDF EF eluates collected for up to $2 \mathrm{wk}$, conferred protection against HIV-1, particularly for the 10 and 20\% formulations (Figure 2.5); whereas HSV-2 inhibition was only achieved using the 1 and $24 \mathrm{~h}$ release eluates (Figure 2.6). The most evident factor that contributes to this lack of efficacy associated with longer release times is the difference in TDF potency against HSV-2 and HIV1. While TDF is efficacious against both HSV-2 and HIV-1, TDF is much less efficacious against HSV-2 $\left(\mathrm{IC}_{50}=8.9 \mu \mathrm{g} / \mathrm{mL}\right)$ relative to HIV-1 $(0.0053 \mu \mathrm{g} / \mathrm{mL})$. Based on the release profiles of the 20\% TDF PLGA and PLCL polymers, we expect that we would need approximately $15-20 \mathrm{mg}$ fiber to provide $3-4 \mathrm{mg}$ of TDF release (over one month), and corresponding efficacy after $2 \mathrm{wk}$ release. This dose corresponds to previous studies indicating that concentrations ranging from 100 to $500 \mu \mathrm{g} / \mathrm{mL}$ are needed to completely prevent HSV-2 replication in vitro ${ }^{56}$. These estimates are within the dosing we envision for in vivo studies, in which similar studies have delivered a range of 0.2 to $0.7 \mathrm{mg} / \mathrm{mL}$ TDF per day within the murine reproductive tract to prevent HIV-1/HSV-2 infections ${ }^{57,69}$.

In addition to the increased amount of TDF needed to prevent HSV-2 relative to HIV-1 infection, the duration of fiber exposure to eluate likely impacts the potency of TDF released from the fibers. This is clearly observed in our efficacy studies where the administration of 1 and $24 \mathrm{~h}$ TDF fiber eluates demonstrated similar efficacy to free TDF; whereas, after $1 \mathrm{wk}$ of release, TDF fiber eluates 
exhibited greater efficacy against HIV-1, relative to free TDF (Table 2.3, Table 2.4).

One factor that supports the improved $I_{50}$ of TDF fibers, relative to free TDF with respect to time, is that TDF is known to hydrolyze to the monoester derivative (mPTFV) in aqueous environments both in vitro and in vivo 56,265,267,268. While increasing the stability of active agents is a benefit of utilizing delivery platforms such as fibers, we acknowledge that even TDF fiber eluate exhibited decreased efficacy against HIV-1 after 1 to 2 wk in media (Table 2.4). HPLC analysis showed that fiber-incorporated TDF was protected from hydrolysis, with no indication of mPTFV accumulation for samples collected during the first week of release. This lack of mPTFV measured in loading and early release samples indicates that the monoester derivative was formed subsequent to fiber release, and that EFs function as an appropriate delivery vehicle to provide drug stability in solution. However, drug that is released and exposed to surrounding fluid for longer durations (here $>1 \mathrm{wk}$ ), will be less efficacious. Additionally, for long-term applications, lactic acid release may enhance TDF hydrolysis and contribute to the decreased efficacy of TDF ${ }^{268}$. Thus, as expected, the longer an incorporated drug remains within the polymer under physiological $\mathrm{pH}$, the longer it will retain efficacy. To modulate release in future work, utilization of a different polymer or polymer blends may more optimally maintain active agent activity against HIV-1 and HSV2 for durations exceeding 1 to 2 weeks.

Finally, the safety of both TDF PLGA and PLCL fibers was assessed after administration to vaginal and cervical cells, and to EpiVaginal tissue. In VK2, Ect1, 
and End1 E6E7 cells, all cell lines demonstrated strong viability after fiber administration for 1 to 3 days (Figure 2.7). This is in agreement with our expectations, given that the polymers have demonstrated biocompatibility and that TDF is FDA-approved. Similarly, after 2 and 3 days exposure to blank or TDF PLGA fibers, EpiVaginal tissue exhibited comparable viability relative to untreated tissue controls. Furthermore, blank PLGA EF- and TDF PLGA EF-treated tissues, revealed normal full thickness vaginal epithelium, with no apparent adverse histological findings. Based on these in vitro results, we expect to see similar safety profiles in in vivo studies.

Another critical aspect of intravaginal delivery is the assessment of inflammatory response. Studies have shown that increased expression of cytokines such as IL-6, IL-8, as well as IL-1 $\alpha$ and $\beta$ is strongly associated with increased susceptibility to HIV-1 infection ${ }^{137,269}$. Nonoxynol-9, once a promising microbicide candidate against HIV-1, was shown to increase the rate of HIV-1 infection in clinical studies due to its pro-inflammatory properties and disruption of the reproductive epithelium ${ }^{270,271}$. Therefore, it is critical that any active agents or delivery vehicles used as a microbicide must minimally induce pro-inflammatory cytokines. For these experiments, PLGA was selected for examination due to the polymer exhibiting both decreased burst release and longer sustained-release properties relative to PLCL fibers. Despite analyzing a plethora of cytokines, TDF EFs were found only to weakly induce (0-2 fold increase) the expression of GMCSF, IL-1 $\alpha$, and IL-6. This cytokine expression was not observed after $72 \mathrm{~h}$, and may have been the result of the actual application of EF onto the EpiVaginal 
tissue $^{252}$. Finally, the nonoxynol-9 control showed a marked decrease of cytokine expression after $72 \mathrm{~h}$, which we attribute to epithelial necrosis and shedding. Previous studies have shown that concentrations of $\mathrm{N}-9$ as low as $0.03 \%$ can induce epithelial disruption and necrosis after $24 h^{272}$. However, no epithelial disruption was observed from TDF EF exposure, indicating that these fibers are non-inflammatory and will not elicit a cytokine response. As in previous studies with microbicides, antiviral compounds may induce cytokine production as high as $3-10$ fold, which is still considered reliably safe ${ }^{69}$.

While our preliminary work with EpiVaginal tissue demonstrates promising biocompatibility, future mechanical testing with respect to the interactions between the fibers, vaginal mucosa, and virus will need to be investigated in vivo. Importantly, tissue contact studies will need to assess how mechanical properties (e.g., flexibility, rigidity, size) impact host tissue interactions. Additionally, while we envision that electrospun fibers may be administered similarly to vaginal films, appropriate studies will need to assess the retention time and distribution of fibers within the vaginal cavity and relate these to the structural properties and mechanical durability after different durations of administration in vivo.

More broadly, the user preference and feasibility of different dosage forms and administration methods must be considered for subsequent clinical development. A variety of studies have highlighted the lack of correlation and reporting of user adherence in clinical trials ${ }^{273,274}$. Hence, there is a need for more accurate reporting and adherence, to fully achieve the prophylactic and/or therapeutic potential of intravaginal delivery vehicles. In particular, if women 
experience user adherence challenges (resulting in unadministered doses), or feel uncomfortable using the dosage form, lower adherence (and efficacy) may result. Given these factors, women's input is critical to the development of microbicide dosage forms that women not only want to use, but are able to use correctly and consistently ${ }^{275}$. Interestingly, recent work that investigated user preferences in vaginal films (for more "on-demand" applications) identified factors-such as the opacity and size of films-that most significantly impacted user preference ${ }^{276}$. As we further develop electrospun fibers for intravaginal applications, we are aware that addressing similar considerations will be necessary to ensure vehicle success in subsequent development stages.

In parallel with these long-term goals of advancing fiber formulations for in vivo studies and clinical applications, in the near-term our laboratory seeks to improve the release profiles, and enhance the efficacy of our electrospun fibers against multiple STIs. The use of different polymers or polymer blends will likely reduce the initial burst release of incorporated products while simultaneously providing for prolonged (>1 wk) release. Additionally while these fibers were not specifically formulated for mucoadhesion, surface-modification or a different polymer choice/blend (e.g., chitosan, acrylic acid polymers) may be considered to improve mucoadhesivity. Furthermore, the development of multilayered and coaxially-spun fibers may also provide a more suitable platform for the delivery of multiple compounds with sustained-release profiles.

\section{Conclusion}


There is an urgent need to develop new and alternative sustained-release technologies to prevent HIV-1 and HSV-2 infections. To address these needs, we fabricated PLGA and PLCL electrospun fibers, and compared the loading and release properties of these fibers, using TDF as a model antiviral. Both PLGA and PLCL fibers provided complete protection against both HIV-1 and HSV-2 infections in vitro. Both short- (1 and $24 \mathrm{~h})$ and long-term release eluates (1 and 2 wk) provided protection against HIV-1; whereas short-term protection (attributed to fiber dosing and difference in $\mathrm{IC}_{50}$ ) was achieved against HSV-2 in vitro. Additionally, TDF fibers demonstrated significantly enhanced efficacy against HIV1 , relative to free TDF, after long-term release of $1 \mathrm{wk}$. Vaginal and cervical cells exposed to TDF PLGA and PLCL fibers showed high viability, after up to 3 days post-administration, demonstrating their safety in vitro. Finally, PLGA fibers induced negligible and temporary increases (0-2 fold) in cytokine expression, suggesting their potential for in vivo applications.

Comparing the attributes of PLGA and PLCL TDF EFs, PLGA appears to be a more promising candidate compared to PLCL, based on its improved release profile. However, as both formulations demonstrated efficacy against HIV-1 and HSV-2 in vitro, future testing may reveal both fiber formulations to be equally efficacious in vivo.

Using the information obtained from this work, we seek to further enhance the efficacy and delivery duration of small molecule antivirals and biologics from EFs by utilizing a variety of encapsulants and polymer blends. In particular, future plans include fabricating formulations that co-deliver multiple active agents. Our 
hope is that these future fibers will provide more potent protection. We predict that these, or similar electrospun fibers will confer long lasting and sustained protection against both HIV-1 and HSV-2 infections. 


\section{CHAPTER 3}

\section{PH-RESPONSIVE DELIVERY OF GRIFFITHSIN FROM ELECTROSPUN FIBERS}

\section{Introduction}

Recently, polymeric electrospun fibers (EFs) have been investigated as a new delivery platform for reproductive applications, demonstrating both ondemand and sustained protection against HSV-2 and HIV-1 infections $^{75,84,86,87,101,161}$. However, one of the challenges of delivery vehicles, including EFs, is that to provide adequate protection they must release therapeutically relevant concentrations of active agents for the duration of use. This often requires frequent administration and highly localized doses to maintain adequate release for prolonged durations. While user adherence may be increased by developing a product that necessitates less frequent application, designing a dosage form that is efficacious regardless of administration time is challenging. Many sustained-release formulations undergo a "burst" release phase, where a significant fraction of active agent is released within the early hours of delivery - regardless of whether this time frame is suitable for protection ${ }^{38}$.

An alternative approach is to design a product that requires less frequent dosing, by inducing the release of active agent only when needed, in response to microenvironmental cues. This strategy has the potential to conserve active agent 
from unnecessary release, provide protection independently of administration time, and deliver active agents directly to the target site of virus entry. One such cue in the reproductive tract, increased $\mathrm{pH}$, is associated with semen infiltration peri- and post-coitus. While the "normal" vaginal $\mathrm{pH}$ ranges from 4.0 to 5.0 , exposure to semen $(\mathrm{pH} \sim 7.5)$ increases the local $\mathrm{pH}$ to more neutral levels. We expect a pH-responsive delivery vehicle that responds to increases in intravaginal $\mathrm{pH}$, will only release active agent when triggered by semen, while maintaining the bioactivity and payload of encapsulated biologics under non-coital conditions.

While $\mathrm{pH}$-responsive delivery has been used in a variety of drug delivery applications $^{79,263,277-281}$, thus far $\mathrm{pH}$-responsive dosage forms are in the early stages of development for delivery to the female reproductive tract (FRT) 83,280,282285. Prior to the use of electrospun fibers for intravaginal applications, temperature and $\mathrm{pH}$ sensitive hydrogels were developed to impart the dual advantages of semen-triggered release and vaginal distribution and retention prior to intercourse 282 . Hydrogels with $\mathrm{pH}$-responsive properties have been shown to release effective concentrations of antivirals. However, hydrogels tend to provide more transient protection due to their propensity for leakage from the FRT. Similarly, polymeric NP platforms comprised of poly(lactic-co-glycolic acid) (PLGA) and S-100 Eudragit $\AA$ blends were evaluated to provide $\mathrm{pH}$-responsive release of the antiretroviral reverse transcriptase inhibitors, tenofovir and TDF ${ }^{283}$. Increased S-100 ratios resulted in decreased encapsulation efficiency, while conversely providing improved $\mathrm{pH}$-dependent release. Similar studies assessed the mucosal delivery of $\mathrm{pH}$-sensitive Eudragit S-100 NPs loaded with hydrophilic or 
hydrophobic molecules ${ }^{280}$, demonstrating retention of molecules within NPs under acidic intravaginal $\mathrm{pH}$ and released upon exposure to more neutral $\mathrm{pH}$ conditions. This study additionally demonstrated the uptake and biocompatibility of NPs in vaginal cells ${ }^{280}$. Most recently, spray dried mucoadhesive and pH-responsive TFV microspheres prepared from polymethacrylate salts were fabricated, resulting in $\sim 90 \%$ release within the first hour, while demonstrating biocompatibility and mucoadhesivity to vaginal cells and porcine vaginal tissue ${ }^{284}$.

Relative to gel and NP delivery platforms, electrospun fibers have recently emerged as an alternative intravaginal delivery platform that offer a durable stationary reservoir of encapsulated agents. However, many of these studies have focused on the delivery of antibiotics or ARVs, relative to new biologics 280,283 . One of the first studies to investigate $\mathrm{pH}$-responsive fibers for vaginal applications, demonstrated that cellulose acetate phthalate (CAP) fibers highly incorporated the reverse transcriptase inhibitors etravirine and TDF, and the hydrophilic dye rhodamine ${ }^{83}$. The CAP polymer, itself a potent antiviral, is minimally soluble in acidic conditions, and the addition of SSF rapidly dissolved the CAP fibers, releasing the encapsulated drugs. While this quick degradation was attributed to the natural (vs. synthetic) polymer chemistry, the fiber degradation raised concerns over long-term structural integrity as well as corresponding protection, prompting the development of fibers with improved mechanical properties. To address this need, coaxial fibers, comprised of a polyurethane core and CAP shell layer, were fabricated to provide $\mathrm{pH}$-inducible release of rhodamine, while demonstrating enhanced mechanical properties ${ }^{142}$. Finally, fibers comprised of Eudragit L-100 
encapsulating horseradish peroxidase and alkaline phosphatase were fabricated using emulsion electrospinning ${ }^{286}$. These fibers modulated protein release in response to $\mathrm{pH}$, while preserving protein activity. In another study, $\mathrm{pH}$-responsive fibers comprised of poly(methacylic acid-co-methyl methacrylate), encapsulating the ARVs, dapivirine and etravirine, were fabricated ${ }^{287}$. These fibers demonstrated sustained-release of therapeutics within acidic conditions, while the fibers rapidly dissolved in alkaline $\mathrm{pH}$, to provide encapsulant release.

While a variety of $\mathrm{pH}$-responsive platforms have demonstrated promise against STIs, many of these platforms, inclusive of electrospun fibers, have focused on the delivery of antibiotics or ARVs, relative to new biologics ${ }^{150,153}$. Recently, we and others have developed EFs as an efficacious platform to provide sustained-delivery of antiviral drugs to the $F R T^{75,84,86,87,101,161}$. Building upon this work, the goal of this project was to develop and test $\mathrm{pH}$-responsive EFs that incorporate the antiviral lectin, GRFT. Griffithsin fibers were designed to address the needs of an on-demand delivery system, while providing a delivery vehicle that may reduce the frequency of daily administration. It is well known that poly(acrylic acid) (PAA) has been used to fabricate a variety of $\mathrm{pH}$-responsive dosage forms ${ }^{288,289}$. Moreover, due to its carboxylic acid groups that are deprotonated within acidic environments (here, vaginal), active agents are retained under slightly acidic conditions. Conversely, in neutral and alkaline environments, the carboxylic acid groups become ionized, inducing electrostatic repulsion, which results in fiber swelling and agent release into the surrounding medium ${ }^{289}$. Additionally, PAA as well as the polymer poly(n-butyl acrylate) (PBA) have been used to produce 
mucoadhesive polymers, demonstrated in buccal delivery and other applications ${ }^{290-295}$. Given these properties, we selected the copolymer, PBA-coPAA, to blend with known sustained-release polymers, PLGA and methoxypolyethylene glycol (mPEG)-PLGA, to provide pH-dependent GRFT release. We hypothesized that the encapsulated GRFT released from these $\mathrm{pH}$ responsive fibers would retain antiviral properties relative to free GRFT and that utilizing PBA-co-PAA fibers to deliver biological entry inhibitors, such as GRFT, may prove useful to conserve the payload and activity of active agent when needed.

\section{Materials and Methods}

\section{Materials}

Carboxyl-terminated poly(d, I-lactic-co-glycolic acid) (PLGA, 50:50, 0.55$0.75 \mathrm{dL} / \mathrm{g}, 31-57 \mathrm{kDa}$ MW) was purchased from LACTEL® Absorbable Polymers (Cupertino, CA, USA). Methoxy poly(ethylene glycol)-b-poly(lactide-co-glycolide) (mPEG-PLGA, 5,000:55,000 kDa) was obtained from PolySciTech® Akina Inc. (West Lafayetter, IN, USA). Poly(n-butyl acrylate-co-acrylic acid) (PBA-co-PAA, 50:50, catalog number 19911-10), an alkali-soluble 20\% latex in alcohol was purchased from Polysciences Inc. (Warrington, PA, USA). Chemical solvents including 1,1,1,3,3,3-hexafluoro-2-propanol (HFIP), dichloromethane (DCM), and hydrochloric acid $(\mathrm{HCl})$ were obtained from Fisher Scientific (Pittsburgh, PA, USA). Sodium dodecyl sulfate (SDS) and MTT [3-(4,5-dimethylthiazol-2-yl)2,5diphenyltetrazolium bromide] were purchased from Sigma Aldrich (St Louis, MO). 
Griffithsin (MW 12.7 kDa) was produced by Kentucky BioProcessing LLC (Owensboro, KY, USA) and was kindly provided by Dr. Kenneth Palmer (University of Louisville). Fetal bovine serum (FBS) and 100x penicillin-streptomycin solutions were purchased from VWR. Simulated vaginal fluid (SVF) and simulated semen fluid (SSF) were prepared as described in ${ }^{246,296}$.

\section{Cell Lines and Virus}

Vaginal keratinocyte (VK2/E6E7), endocervical (End1/E6E7), and ectocervical (Ect1/E6E7) cell lines were used to assess fiber cytotoxicity (courtesy of Dr. Kenneth Palmer, originally from ATCC, Rockville MD). VK2/E6E7 (VK2), End1/E6E7 (End1), and Ect1/E6E7 (Ect1) are well-characterized immortalized cell lines derived from normal human vaginal, endocervical, and ectocervical epithelia, respectively. These cell lines were chosen as they are representative of the cell types found within the female reproductive tract. VK2, End1, and Ect1 cells were maintained in keratinocyte serum-free medium (KSFM) supplemented with recombinant human epidermal growth factor $(0.1 \mathrm{ng} / \mathrm{mL})$, bovine pituitary extract $(50 \mu \mathrm{g} / \mathrm{mL})$, calcium chloride $(0.4 \mathrm{mM})$ (Thermo Fisher, Waltham, MA), with $1 \%$ penicillin and streptomycin $(100 \mu \mathrm{g} / \mathrm{mL}$ each). During cell trypsinization, plating, and cell counting, cells were neutralized with Dulbecco's Modified Eagle Medium: Nutrient Mixture F-12 media (DMEM/F-12, 1:1, VWR) with 10\% fetal bovine serum (FBS), and $1 \%$ penicillin/streptomycin $(100 \mu \mathrm{g} / \mathrm{mL}$ each $)$.

TZM-bl cells, obtained from the National Institutes of Health AIDS Research and Reference Reagent Program (ARRRP), were used to assess in vitro HIV-1 
infectivity. TZM-bl cells, previously designated JC53-bl (clone 13), are derived from a HeLa cell clone engineered to express CD4, CCR5 and CXCR4. These cells have a Tat-driven luciferase or E. coli $\beta$-galactosidase reporter system, under the control of an HIV-1 long terminal repeat, permitting sensitive and accurate measurements of infection 247,248 . TZM-bl cells were cultured in DMEM containing $10 \%$ FBS, $25 \mathrm{mM}$ HEPES, and $50 \mu \mathrm{g} / \mathrm{mL}$ gentamicin. These cells are highly permissive to infection by most strains of HIV-1, SIV and SHIV, including primary or molecularly cloned viral isolates and molecularly cloned Env-pseudotyped viruses.

The Env-pseudotype HIV-1 was produced in $293 \mathrm{~T} / 17$ cells, using an envelope (env)-expressing plasmid (CCR5-tropic clade A strain, Q769.h5) and an env-deficient HIV-1 backbone vector ( $p N L 4.3 \Delta E n v-L u c$ ), both obtained from the NIH AIDS Reagent Program (11884 and 3418). HEK-293T (human embryonic kidney) cells were purchased from ATCC. Cells were maintained in MEM supplemented with $10 \%$ FBS, and $1 \%$ penicillin and streptomycin $(100 \mu \mathrm{g} / \mathrm{mL}$ each).

\section{Synthesis of Electrospun Fibers}

Blank PLGA and mPEG-PLGA polymers $(15 \% \mathrm{w} / \mathrm{w})$ were dissolved in $0.6 \mathrm{~mL}$ HFIP overnight, while shaking at room temperature. Subsequent polymer blends, comprised of varying PLGA:PBA-co-PAA polymer ratios (100:0, 90:10, $85: 15,80: 20$, and $75: 25 \mathrm{w} / \mathrm{w}$ ) were prepared as follows (Figure 3.1). Briefly, PLGA polymers $(15-30 \% \mathrm{w} / \mathrm{w}$ ) were first dissolved in HFIP. Corresponding mass to mass 
ratios of PBA-co-PAA polymer were added to the PLGA polymer mixture and allowed to solubilize overnight on a shaker at room temperature. Prior to electrospinning, $140 \mu \mathrm{L}$ of Tris-EDTA buffer (TE, $\mathrm{pH}=7.4$ ) was added to the polymer solution and briefly vortexed. 


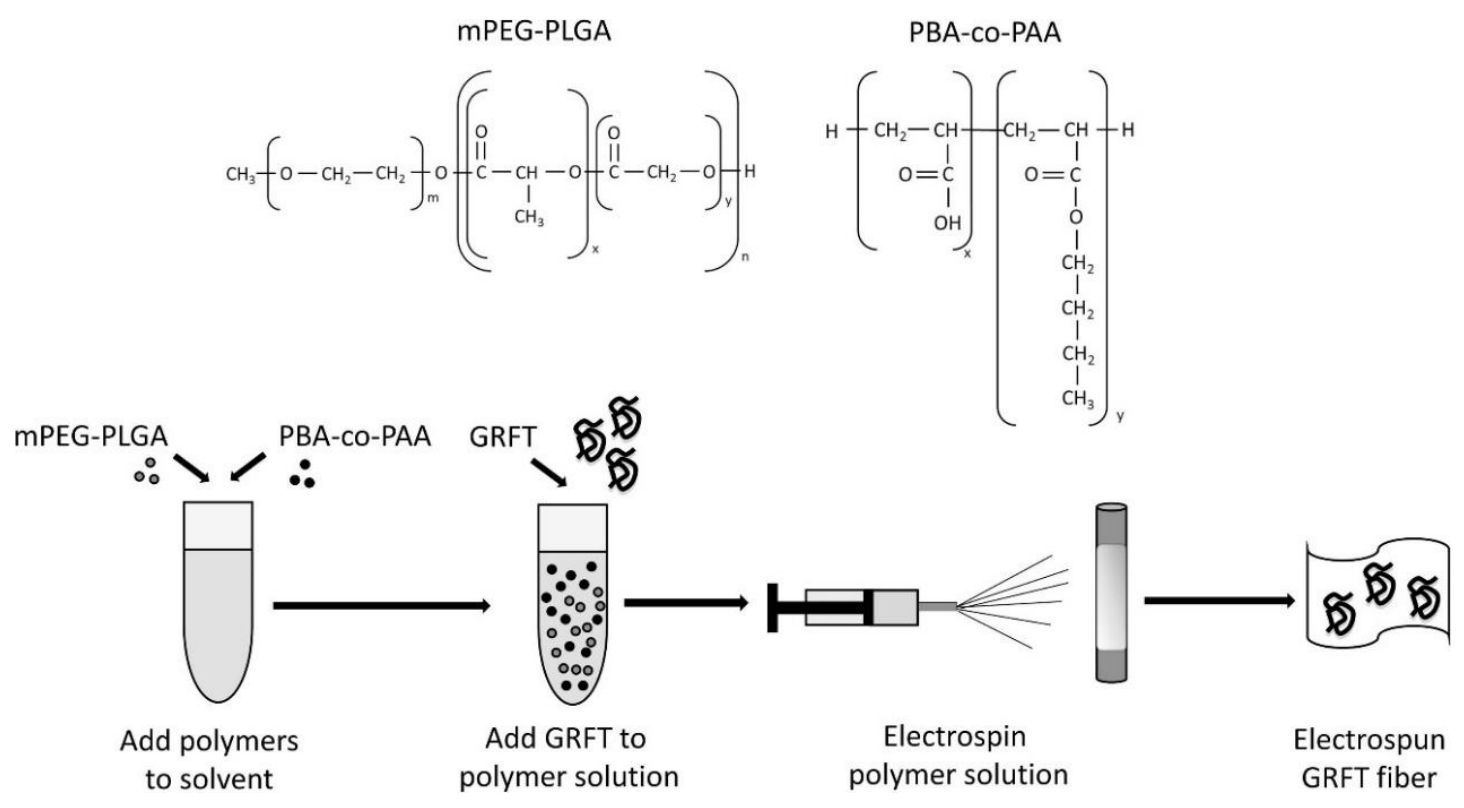

Figure 3.1 Schematic of mPEG-PLGA and PBA-co-PAA co-polymers and the fiber fabrication process. 
For fibers incorporating GRFT, the GRFT stock solution was first concentrated with a Spin-X® UF concentrator (10 kDa MWCO, Corning Incorporated-Life Sciences, Oneonta, New York, USA) and resuspended to a volume of $1 \mathrm{~mL}$ in TE buffer. For a 90:10 polymer blend, $144 \mathrm{mg}$ of either PLGA or mPEG-PLGA was weighed and added to $0.6 \mathrm{~mL}$ of HFIP. Immediately after, $80 \mu \mathrm{L}$ $(16 \mathrm{mg})$ of PBA-co-PAA ethanol solution $(20 \% \mathrm{w} / \mathrm{v})$ was added to this mixture, resulting in a polymer blend with a final concentration of $13.8 \% \mathrm{w} / \mathrm{w}$ (polymer/solvent). After the polymers solubilized overnight, $140 \mu \mathrm{L}(4.8 \mathrm{mg})$ of GRFT solution $(34.2 \mathrm{mg} / \mathrm{mL})$ was added dropwise to the polymer mixture giving a final electrospinning volume of $820 \mu \mathrm{L}$, with theoretical GRFT loading of $30 \mu \mathrm{g}$ GRFT/mg polymer. The solution was briefly vortexed and immediately electrospun.

Five hundred microliters of PLGA:PBA-co-PAA and GRFT suspension was aspirated into and electrospun from a $1 \mathrm{~mL}$ plastic syringe as previous described ${ }^{87,161}$. Flow rates spanning $(0.3-1.0 \mathrm{~mL} / \mathrm{h})$ were optimized over a range of voltages $(20-25 \mathrm{kV})$. The resulting fiber mat was collected on a rotating $4 \mathrm{~mm}$ outer diameter stainless steel grounded mandrel located $10-20 \mathrm{~cm}$ from the needle tip. All electrospinning processes were performed at room temperature (RT, $\sim 25^{\circ} \mathrm{C}$ ). Sample flow rate was monitored using an infusion pump (Fisher Scientific, Pittsburgh, PA) while the voltage was applied using a high voltage power supply (Spellman CZE 1000R). Final electrospinning conditions applied a voltage of $25 \mathrm{kV}$, with a sample flow rate of $0.8 \mathrm{~mL} / \mathrm{h}$, with a distance of $20 \mathrm{~cm}$, for the 
mPEG-PLGA:PBA-co-PAA 90:10 blend. After electrospinning, fibers were removed from the mandrel and dried overnight in a desiccator.

\section{Fiber Morphology}

The morphologies of GRFT PLGA:PBA-co-PAA and mPEG-PLGA:PBA-coPAA blended fibers were evaluated using scanning electron microscopy (SEM) (JSM-820 microscope, JEOL, Tokyo, Japan). Briefly, after drying, fibers were cut into $5 \mathrm{~mm}$ pieces and placed on double-sided adhesive carbon tabs (Ted Pella, Inc., Redding, CA, USA), which were then adhered to aluminum stubs. Samples were coated with a thin gold alloy film using a Bio-Rad E5100 sputter coat system. The coating process was operated at $20 \mathrm{~mA}$ for $90 \mathrm{~s}$ and images were captured at an accelerating voltage of $8 \mathrm{kV}$. The average fiber diameter was determined by analyzing SEM images in NIH ImageJ, and drawing line elements across a minimum of 50 fibers per image. Statistical significance between fiber diameters was determined using the Bonferroni post hoc t-test $(p<0.05)$.

\section{Griffithsin Loading and Release}

The amount of GRFT incorporated into each fiber was determined by dissolving of 3-5 mg fiber pieces in $500 \mu \mathrm{L} \mathrm{DCM}$, and adding $500 \mu \mathrm{L}$ TE buffer to extract GRFT. This extraction process was repeated twice by vortexing for $1 \mathrm{~min}$ and centrifuging for $5 \mathrm{~min}$ at $13,000 \mathrm{rpm}$ to fully extract GRFT. The TE buffer was collected and analyzed using ELISA to determine GRFT loading. The encapsulation efficiency (EE) was defined as the ratio of actual GRFT loading to the theoretical loading $(30 \mu \mathrm{g} / \mathrm{mg})$. 
To quantify GRFT release from $\mathrm{pH}$-responsive fibers under different in vitro conditions, SVF $(\mathrm{pH}=4.4)$, phosphate buffered saline (PBS, $\mathrm{pH}=7.4)$, and SVF:SSF $(1: 3, v / v, p H=7.4)$ were used as eluates. The SVF:SSF mixture was used to measure inducible GRFT release after exposure to semen-vaginal fluid mixture $^{296}$. Samples of GRFT PLGA:PBA-co-PAA and mPEG-PLGA:PBA-co-PAA fibers were hole-punched with a Ribbel biopsy punch ( $7 \mathrm{~mm}$ diameter), resulting in an approximate fiber mass of $10 \mathrm{mg}$. These fibers were immersed in $1 \mathrm{~mL}$ SVF or PBS, in a shaker at $37^{\circ} \mathrm{C}$ and $150 \mathrm{rpm}$. At pre-determined time points, the release buffer was completely replaced with $1 \mathrm{~mL}$ of either fresh SVF or PBS. In subsequent $\mathrm{pH}$-dependent release studies, fibers were incubated in SVF for 24, 48, or $72 \mathrm{~h}$. To create a pH "switch", the SVF was replaced with either PBS or (1:3) SVF:SSF to assess differences in GRFT release after a $\mathrm{pH}$ change. Fiber eluates were collected and replaced at 1, 4, 24, 48, 72, 96, and $120 \mathrm{~h}$ after the initial PBS or SVF:SSF "switch."

The concentration of GRFT released was determined using an established ELISA method. Briefly, Nunc Maxisorp ELISA plates were coated with $0.9 \mathrm{mg} / \mathrm{mL}$ influenza virus hemagglutinin (diluted in $0.1 \mathrm{M} \mathrm{PBS}$ ) overnight at $4{ }^{\circ} \mathrm{C}$ for use as a coating buffer, which GRFT selectively binds to. The plate was washed three times with PBS containing 0.05\% Tween-20 (PBST) using an Immunowash plate washer (Bio-Rad, Hercules, CA, USA). The wells were blocked by adding $3 \%(w / v)$ bovine serum albumin in PBST at room temperature (RT) for $2-3 \mathrm{~h}$ to block non-specific binding. A GRFT standard, loading extract and release eluates were added to the wells for $1 \mathrm{~h}$ at room temperature. A 1:10,000 dilution of both a primary antibody 
goat anti-GRFT (provided by Dr. Nobuyuki Matoba, University of Louisville) and secondary antibody rabbit anti-goat IgG-HRP (Sigma-Aldrich, St. Louis, MO, USA) were added to the wells for another $1 \mathrm{~h}$ to detect bound GRFT. Colorimetric values were derived using SureBlue Reserve TMB Peroxidase substrate (KPL, Gaithersburg, MD, USA), and the reactions were stopped by $1 \mathrm{~N} \mathrm{H} 2 \mathrm{SO} 4$. Absorbance was measured at $450 \mathrm{~nm}$ on a Synergy HT reader (BioTek, Winooski, VT, USA). Results are shown as the cumulative amount of GRFT released per mass fiber $(\mu \mathrm{g} / \mathrm{mg})$ and the cumulative release percentage, as a function of release time. The encapsulation efficiency (EE) was defined as the ratio of actual GRFT loading to the theoretical loading $(30 \mu \mathrm{g} / \mathrm{mg})$. All data are shown as the mean \pm standard deviation. All experiments were conducted in triplicate, with a minimum of three independent experiments, unless otherwise noted.

\section{Nuclear Magnetic Resonance Spectroscopy}

For the quantification of the polymer composition, mPEG-PLGA, PLGA-coPAA, empty-fiber, and GRFT-loaded fiber were dissolved with fully deuterated dimethyl sulfoxide for Nuclear magnetic resonance (NMR) spectroscopy. NMR spectra of all the samples were obtained at a temperature of $298 \mathrm{~K}$ using a $600 \mathrm{MHz}$ proton frequency spectrometer equipped with a triple resonance prodigy probe (Bruker, Billerica, MA, USA). All the spectra were acquired with 2048 complex points and 4 number of scans and processed using TOPSPIN. Based on the chemical structures of mPEG-PLGA and PBA-co-PAA, peaks in the spectra of mPEG-PLGA and PBA-co-PAA were assigned to individual proton resonances. These assignments were consequently used to identify resonances in the fiber 
spectra. Although severe overlapped resonances from mPEG moiety in mPEGPLGA and aliphatic moiety in PBA-co-PAA in the fiber spectra were observed, protons attached to carbons of lactic and glycolic moieties in MPEG-PLGA and proton in hydroxyl group in PBA-co-PAA could be unambiguously assigned to the resonances at $\sim 5.0, \sim 5.5$, and $\sim 8.0 \mathrm{ppm}$, respectively (Supplemental Figure 3.1). After the assignments, the relative composition of moieties in mPEG-PLGA were determined using integration of resonances in each spectrum and the determined number of chains for lactic and glycolic moieties in mPEG-PLGA were $\sim 34$ and $\sim 54$, respectively and the chain number of acrylic acid in PBA-co-PAA was $\sim 34$. The relative ratio of $\mathrm{mPEG}-\mathrm{PLGA}$ and PBA-co-PAA in the fiber were determined as 1:0.1 using the integration of the peaks at $\sim 5.0, \sim 5.5$, and $\sim 8.0 \mathrm{ppm}$ in the spectra of fibers (Supplemental Figure 3.1). Additional to the resonances from the fiber, GRFT resonances were observed only in the GRFTloaded fiber (Supplemental Figure 3.1) that confirms that GRFT is incorporated into the fiber using our protocol. 

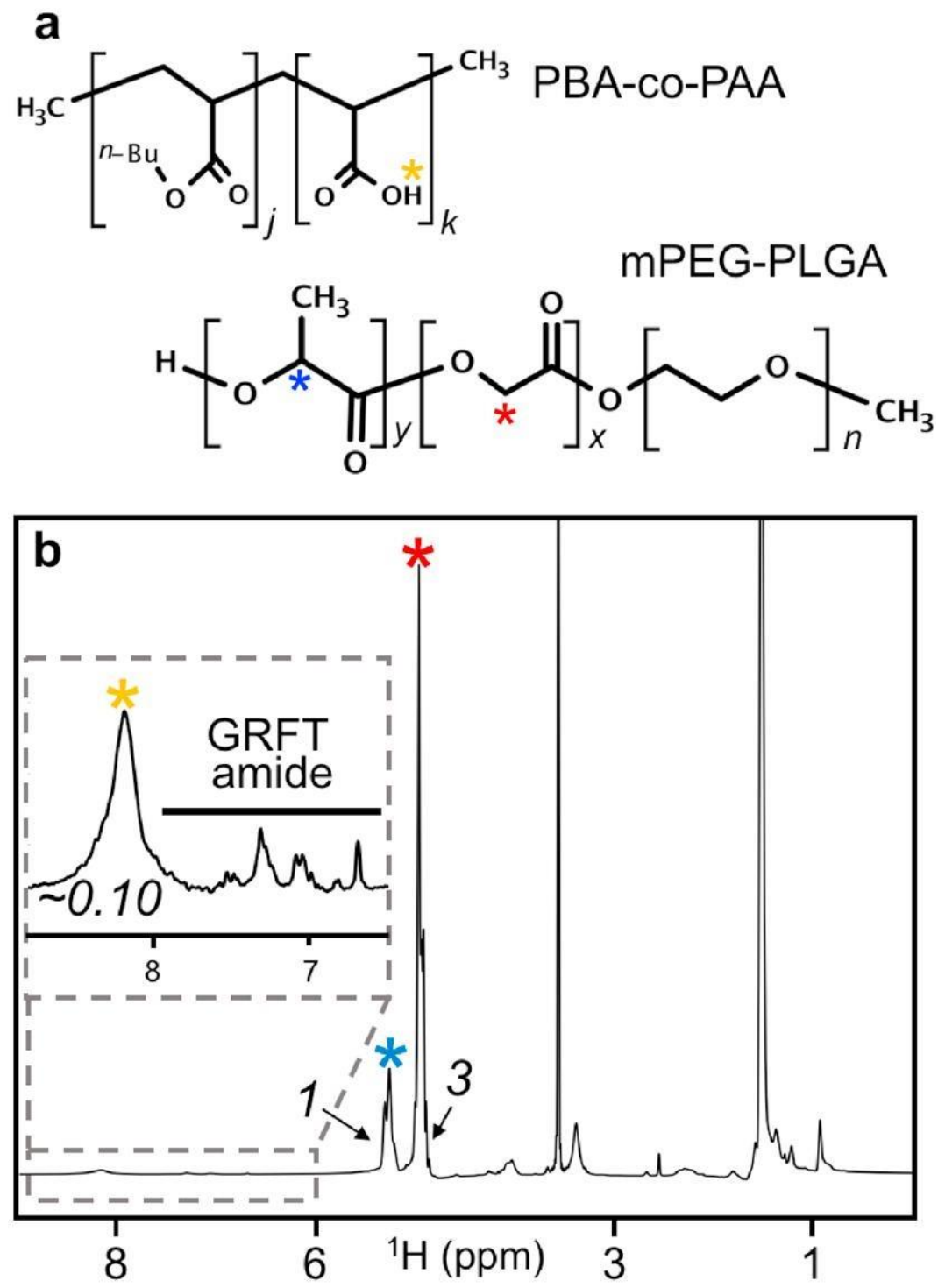

Supplemental Figure 3.1 NMR Spectrograph of GRFT Fibers. 


\section{HIV-1 Pseudovirus-Based Neutralization Assay}

The antiviral activity of GRFT loading extract and fiber release eluates was measured relative to free GRFT, as a function of reduction in luciferase reporter gene expression after a single round of infection with Env-pseudotyped virus (CCR5-using clade A strain Q769.h5) in TZM-bl cells. The optimal virus dilution was established to yield $\geq 100,000$ relative luminescence units (RLU) after infection. Assay stocks of molecularly cloned Env-pseudotyped viruses were prepared by transfection of $293 \mathrm{~T}$ cells and were titrated in TZM-bl cells as previously described ${ }^{297}$. Briefly, all samples were diluted using serial dilutions (ranging from no dilution to $1: 10,000$ with PBS to a final volume of $50 \mu \mathrm{L}$ within a 96-well plate. One hundred microliters of TZM-bl cell solution (104 cells in DMEM medium with $10 \mu \mathrm{g} / \mathrm{mL}$ DEAE-dextran) was subsequently added to each well, followed by the addition $50 \mu \mathrm{L}$ of HIV-1 pseudovirus virus dilution. Samples were then incubated at $37^{\circ} \mathrm{C}$ for $48 \mathrm{~h}$. After the $48 \mathrm{~h}$ incubation, $100 \mu \mathrm{L}$ culture medium was carefully removed from each well. Luminescence was measured using the Bright-Glo ${ }^{\mathrm{TM}}$ luciferase assay system (Promega Corporation, Madison, WI, USA) by adding $100 \mu \mathrm{L}$ Bright-Glo ${ }^{\mathrm{TM}}$ reagent solution to each well for $5 \mathrm{~min}$. Plates were read via luminescence by the Synergy HT reader (BioTek, Winooski, VT, USA). All $R L U$ values were corrected by subtracting the RLU of untreated/uninfected cells from the sample RLUs (treated infected cells). The percent virus inhibition was determined by normalizing the corrected RLUs of infected/treated cells to corrected untreated/infected cells: \% Infection $=[($ sample RLU - untreated uninfected cells $) \div$ (untreated infected cells - untreated uninfected cells) $] \times 100 \%$. 
Antiviral activity is reported as the sample concentration at which RLUs compared with virus control wells RLUs.

\section{In Vitro Cytotoxicity}

The in vitro cytotoxicity of mPEG-PLGA:PBA-co-PAA (90:10), relative to free GRFT was evaluated in VK2, Ect1, and End1 cells using a colorimetric MTT assay. Briefly, each cell line was seeded into 12 -well plate at a density of 600,000 cells per well. Eluates from 1 and 10 mg GRFT mPEG-PLGA:PBA-co-PAA fibers, suspended in $1 \mathrm{~mL}$ of media were incubated with the cells for 24,48 , and $72 \mathrm{~h}$. After each time point, $100 \mu \mathrm{L}$ MTT solution $(5 \mathrm{mg} / \mathrm{mL})$ was added to each well followed by incubation for $4 \mathrm{~h}$ at $37^{\circ} \mathrm{C}$. Lysis buffer (550 $\mu \mathrm{L}, 10 \%$ SDS in $0.01 \mathrm{M}$ $\mathrm{HCl}$ ) was then added to each well for and incubated for $16 \mathrm{~h}$. Absorbance readings

were performed at $570 \mathrm{~nm}$. Ten percent DMSO was used as the positive control for cytotoxicity, with blank fibers eluate-treated and untreated cells as negative controls. All data are shown as the mean \pm standard deviation.

\section{Results}

\section{Fiber Morphology}

The morphology and microstructure of PLGA and mPEG-PLGA fibers were assessed with scanning electron microscopy (Figure 3.2). In addition, the morphology of PLGA and PBA-co-PAA polymer fibers with blend ratios of: 100:0, 90:10, 85:15, 80:20 and 75:25 and mPEG-PLGA:PBA-co-PAA (90:10) fibers were evaluated (Figure 3.3). As shown in Figure 3.2 and Figure 3.3, all formulations provided well-defined fiber morphologies. The fiber diameters of the different 
polymer blends tested for $\mathrm{pH}$-responsive release were assessed using ImageJ software (NIH) (Table 3.1). The fiber diameters of the PLGA:PBA-co-PAA and mPEG-PLGA:PBA-co-PAA (90:10) blends ranged from 204 to $407 \mathrm{~nm}$. As the initial PBA-co-PAA ratio increased, the average fiber diameter decreased; however, only the 75:25 PLGA blend exhibited a statistically significant difference relative to the other PLGA blends. There was no evident relationship between polymer type, PBA-co-PAA ratio, and fiber diameter. 

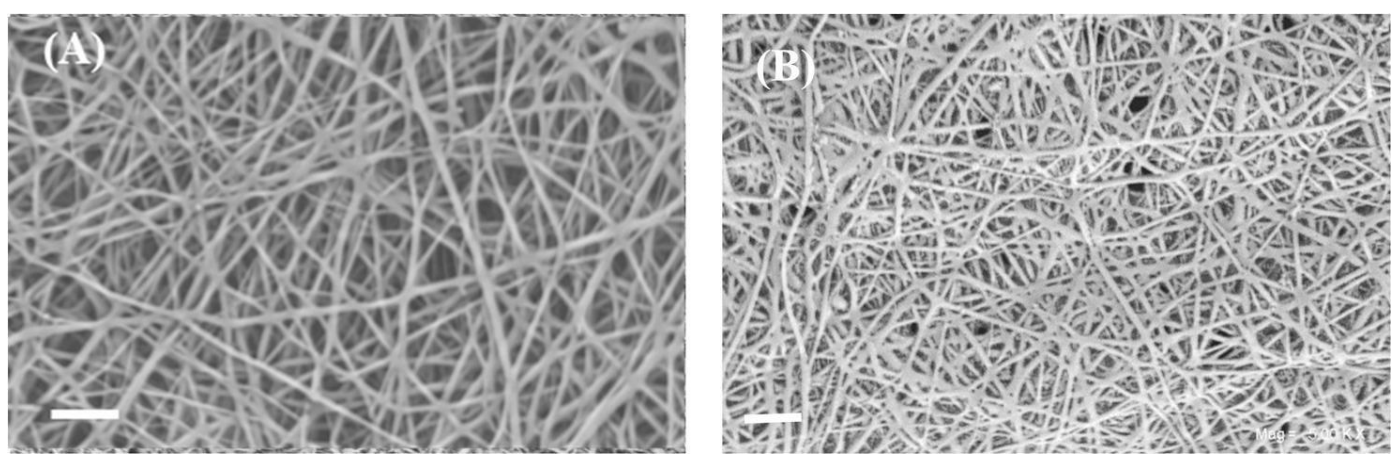

Figure 3.2 SEM images of (A) PLGA and (B) mPEG-PLGA electrospun polymer fibers that incorporate GRFT. The scale bar represents $2 \mu \mathrm{m}$. 


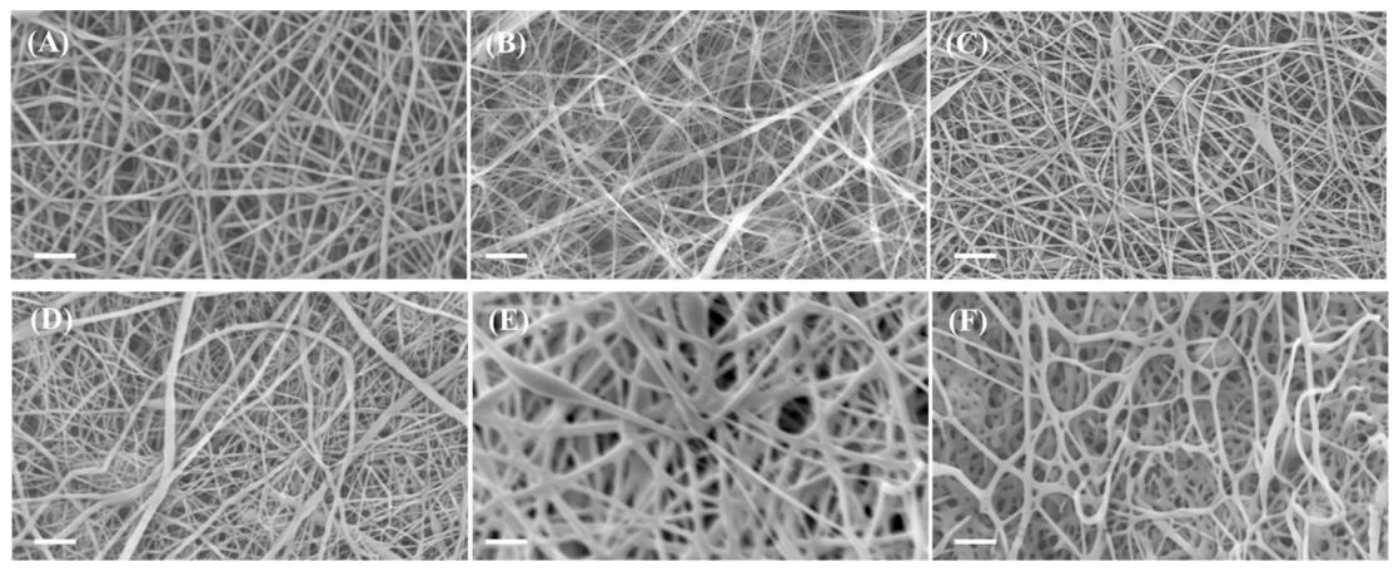

Figure 3.3 SEM images of different $\mathrm{pH}$-responsive electrospun fiber blends that incorporate GRFT. (A) PLGA:PBA-co-PAA (100:0, w/w); (B) PLGA:PBA-co-PAA (90:10); (C) PLGA:PBA-co-PAA (85:15); (D) PLGA:PBA-co-PAA (80:20); (E) PLGA: PBA-co-PAA (75:25); and (F) mPEG-PLGA:PBA-co-PAA (90:10). The scale bar represents $2 \mu \mathrm{m}$. 
Table 3.1 pH-responsive fiber diameters measured from SEM images.

\begin{tabular}{|c|c|c|}
\hline Fiber Formulation & Weight Ratio & Fiber Diame ter (nm) \\
\hline \multirow{3}{*}{ PLGA:PBA-co-PAA } & $100: 0$ & $263 \pm 81$ \\
\cline { 2 - 3 } & $90: 10$ & $236 \pm 82$ \\
\cline { 2 - 3 } & $85: 15$ & $239 \pm 106$ \\
\cline { 2 - 3 } & $80: 20$ & $204 \pm 104$ \\
\cline { 2 - 3 } & $75: 25$ & $407 \pm 150$ \\
\hline mPEG-PLGA:PBA-co-PAA & $90: 10$ & $336 \pm 100$ \\
\hline
\end{tabular}




\section{Griffithsin Fiber Loading and Release Characterization}

To determine the impact of polymer blend ratio on GRFT loading, the extracts from 100:0, 90:10, 85:15, 80:20 and 75:25 PLGA:PBA-co-PAA and 90:10 mPEG-PLGA:PBA-co-PAA polymer blends were evaluated using an ELISA. Table

3.2 compares the actual amount of GRFT incorporated per milligram of fiber, to the theoretical loading of $30 \mu \mathrm{g} / \mathrm{mg}$. The encapsulation efficiency of active GRFT in PLGA-only (100:0) EFs, measured via ELISA was over 90\%, indicating the high loading potential of these fibers. The 90:10, 85:15, 80:20 and 75:25 PLGA:PBAco-PAA blends exhibited GRFT encapsulation efficiencies of $62,53,51$, and $80 \%$, respectively, while the 90:10 mPEG-PLGA:PBA-co-PAA fibers demonstrated 54\% loading efficiency. Similarly high GRFT loading was attained for both the PLGAonly and the $75: 25$ polymer blend, while GRFT loading of the other formulations was statistically lower. Although statistical significance was observed between the encapsulation efficiencies of different fiber formulations, there was no correlation between GRFT loading and increased PBA-co-PAA ratio. 
Table 3.2 GRFT loading and encapsulation efficiency based on formulation.

\begin{tabular}{|c|c|c|c|c|}
\hline \multicolumn{5}{|c|}{ Quantification of GRFT EF Loading and Encapsulation Efficiency } \\
\hline Fiber Formulation & Weight Ratio & $\begin{array}{l}\text { Theoretical Loading } \\
(\mu \mathrm{g} / \mathrm{mg})\end{array}$ & $\begin{array}{c}\text { Actual Loading } \\
(\mu \mathrm{g} / \mathrm{mg})\end{array}$ & \begin{tabular}{|c} 
Encapsulation Efficiency \\
$(\%)$
\end{tabular} \\
\hline \multirow{5}{*}{ PLGA:PBA-CO-PAA } & $100: 0$ & \multirow{6}{*}{30} & $29.6 \pm 1.6$ & $98.7 \pm 5.0$ \\
\hline & 90:10 & & $18.7 \pm 0.9$ & $62.3 \pm 2.8$ \\
\hline & $85: 15$ & & $16.0 \pm 3.8$ & $53.4 \pm 12.9$ \\
\hline & $80: 20$ & & $14.5 \pm 1.8$ & $51.3 \pm 6.1$ \\
\hline & $75: 25$ & & $24.0 \pm 0.8$ & $80.0 \pm 2.5$ \\
\hline mPEG-PLGA:PBA-Co-PAA & $90: 10$ & & $16.2 \pm 1.2$ & $54.0 \pm 13.7$ \\
\hline
\end{tabular}


To initially assess the ability of fibers to release GRFT under different $\mathrm{pH}$ conditions, the total cumulative release of GRFT from PLGA and mPEG-PLGA fibers was measured during $72 \mathrm{~h}$ incubation in SVF ( $\mathrm{pH} 4.4)$ or PBS $(\mathrm{pH} 7.4)$. Although PLGA EFs demonstrated high GRFT encapsulation, negligible GRFT release was observed from PLGA-only fibers in either SVF or PBS (Figure 3.4). However, GRFT release from mPEG-PLGA fibers in SVF and PBS exhibited burst release within the first $6 \mathrm{~h}$ ( $\sim 33$ and $45 \%$ of total GRFT loading, respectively), followed by minimal sustained-release during the remaining incubation period (Figure 4.4). Based on these results, alternative polymer formulations were investigated to improve GRFT release. 


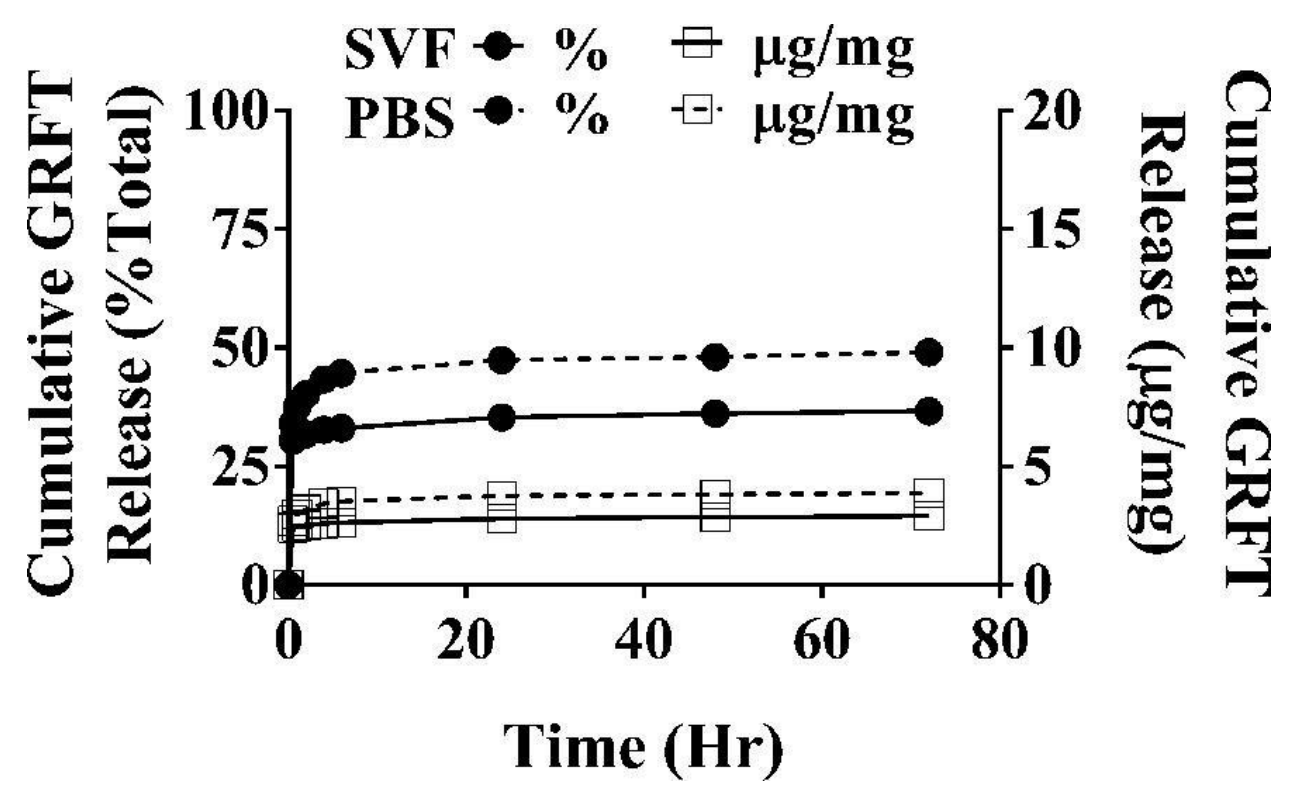

Figure 3.4 In vitro release profiles of GRFT from mPEG-PLGA fibers. Negligible GRFT release was detected from PLGA-only fibers (data not shown, release coincides with $\mathrm{x}$-axis). Cumulative release data is shown as a function of percent total loading (solid circles) and mass released per mass of fiber (open squares). Fibers were incubated in either SVF (solid lines) or PBS (dashed lines) for $72 \mathrm{~h}$ at $37^{\circ} \mathrm{C}$. Data are expressed as the mean \pm SD of triplicate samples. 
In comparison to these initial release studies with PLGA and mPEG-PLGA fibers, it was observed that blending PLGA with a $\mathrm{pH}$-responsive polymer, PBAco-PAA, both enabled the formation of well-delineated fibers (Figure 4.3), and provided enhanced GRFT release in PBS, relative to PLGA- or mPEG-PLGA-only fibers (Figure 3.5). To mimic the more basic $\mathrm{pH}$ conditions of semen entry for $\mathrm{pH}$ responsive applications, EFs were incubated in SVF for $24 \mathrm{~h}$, and subsequently "switched" to PBS (Figure 3.5). The same fiber formulations were also incubated in PBS alone as a control. While unblended PLGA-only fibers (100:0) released minimal amounts of GRFT, polymer blends containing increased PBA-co-PAA $(10-25 \% \mathrm{w} / \mathrm{w})$, released more GRFT within the first $24 \mathrm{~h}$ in either PBS or SVF. Of these blends, only the 90:10 PLGA:PBA-co-PAA blend exhibited increased GRFT release in PBS only (relative to SVF), indicating its potential for $\mathrm{pH}$-responsive applications. In contrast, blends with increased PBA-co-PAA ratios (15, 20, and 25\%) released GRFT in both PBS as well as SVF, deeming them less discerning for a $\mathrm{pH}$-responsive delivery platform. 

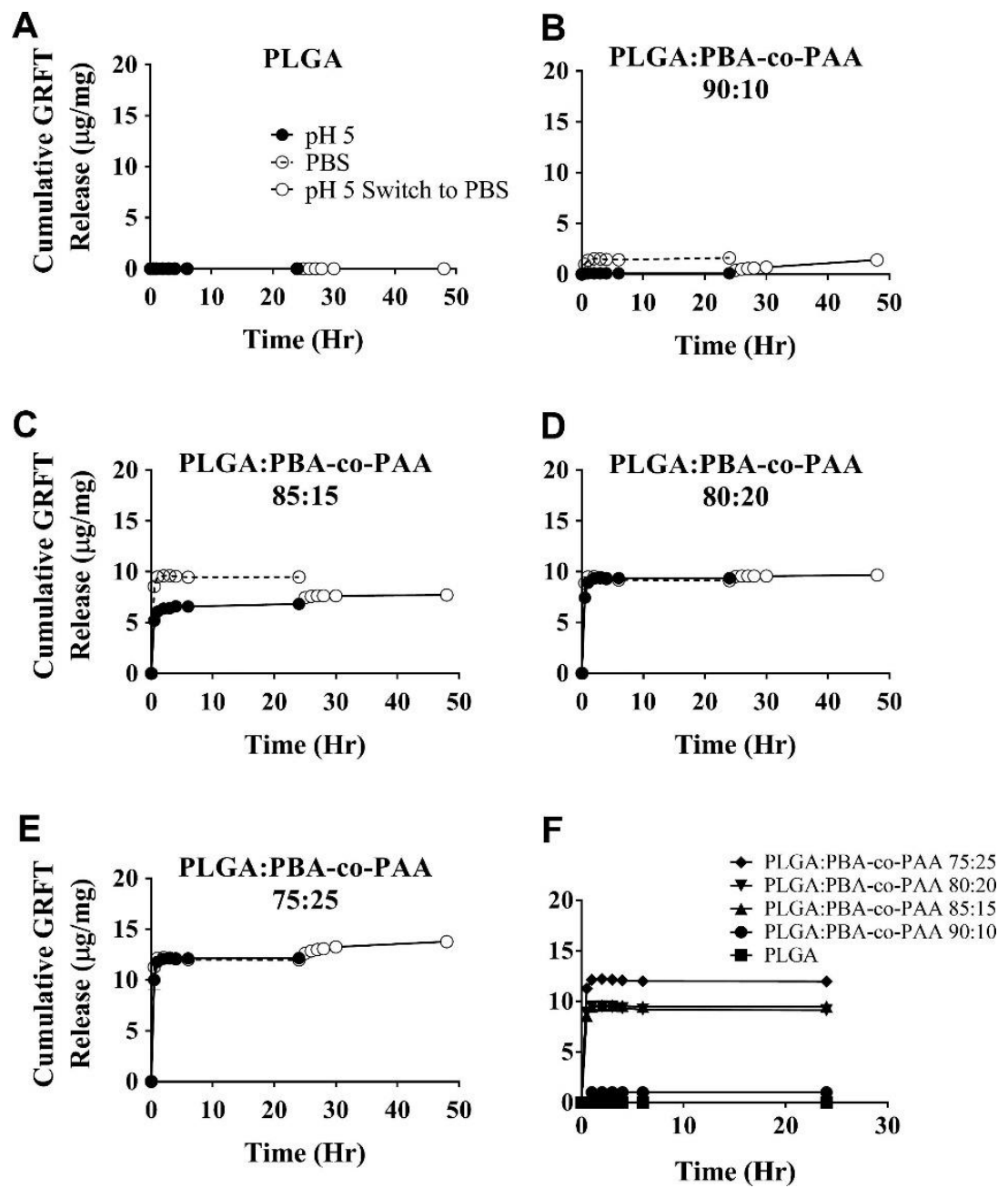

Figure 3.5 In vitro pH-responsive release profiles of GRFT from different PLGA:PBA-co-PAA blended fiber formulations, each theoretically loaded with $30 \mu \mathrm{g} \mathrm{GRFT/mg} \mathrm{polymer.} \mathrm{The} \mathrm{cumulative} \mathrm{release} \mathrm{of} \mathrm{GRFT} \mathrm{from:} \mathrm{(A)} \mathrm{PLGA:PBA-}$ co-PAA (100:0, w/w); (B) PLGA:PBA-co-PAA (90:10); (C) PLGA:PBA-co-PAA (85:15); (D) PLGA:PBA-co-PAA (80:20); and (E) PLGA: PBA-co-PAA (75:25) were monitored at $37^{\circ} \mathrm{C}$. Fibers were initially incubated in SVF $(\mathrm{pH}=4.5)$ for $24 \mathrm{~h}$ and switched to PBS $(\mathrm{pH}=7.4)$ for an additional $24 \mathrm{~h}$. Release in PBS $(\mathrm{pH} 7.4)$ is shown (without a switch) for comparison. A summary of GRFT release from all blends in PBS is provided in (F). As the ratio of PLGA decreases, the cumulative 
amount of GRFT release increases. Release data are expressed as the mean \pm SD of triplicate samples. 
Based on these release results (Figure 3.4, Figure 3.5), it was hypothesized that incorporating the properties of the 90:10 PLGA:PBA-co-PAA blend with the increased hydrophilicity of mPEG-PLGA, may increase GRFT release in more neutral conditions due to increased hydrophilicity, while retaining GRFT loading at low intravaginal pH (e.g., SVF). To evaluate this, mPEGPLGA:PBA-co-PAA (90:10) fibers were formulated and incubated in SVF for either 24, 48, and $72 \mathrm{~h}$. After 24, 48, and $72 \mathrm{~h}$, the fibers were "switched" to PBS (Figure 3.6A) or simulated vaginal-semen fluid (SVF:SSF (1:3) w/w, pH 7.4)) (Figure 3.6B). The mPEG-PLGA:PBA-co-PAA blend provided negligible release of GRFT in SVF after 24,48 , and $72 \mathrm{~h}$, releasing only $0.27,0.41$, and $0.47 \mu \mathrm{g} / \mathrm{mg}$ GRFT respectively. However, when switched to PBS, the blend exhibited a nearly thirtyfold increase in GRFT release, releasing $10-12 \mu \mathrm{g} / \mathrm{mg}$ (66-74\%) over a subsequent $120 \mathrm{~h}$ (Figure 3.6A). Moreover, within the first hour post-switch, the fibers that were incubated for 24,48 , and $72 \mathrm{~h}$ in SVF released 6.2, 3.5, and $4.2 \mu \mathrm{g}$ GRFT/mg fiber, respectively. Similar release was observed when fibers were switched from SVF to a more representative intravaginal environment of SVF:SSF (1:3), with 1.3, 1.3, and 1.4 $\mu \mathrm{g}$ GRFT/mg fiber release $1 \mathrm{~h}$ post-switch from the 24, 48, and 72 h SVF incubations, respectively (Figure 3.6B). Overall, GRFT release from fibers switched to SVF:SSF showed a lesser and more gradual release curve relative to fibers switched to PBS. While fibers switched to PBS released approximately $11 \mu \mathrm{g}$ GRFT/mg fiber $24 \mathrm{~h}$ post-switch, fibers switched to SVF:SSF released $\sim 5 \mu \mathrm{g}$ GRFT/mg fiber within the same duration. Moreover, the PBS switched fibers exhibited minimal release after $24 \mathrm{~h}$, while the SVF:SSF switched 
fibers continued to release, resulting in a total of $\sim 7 \mu \mathrm{g}$ GRFT released per mg fiber, $72 \mathrm{~h}$ post-switch. 

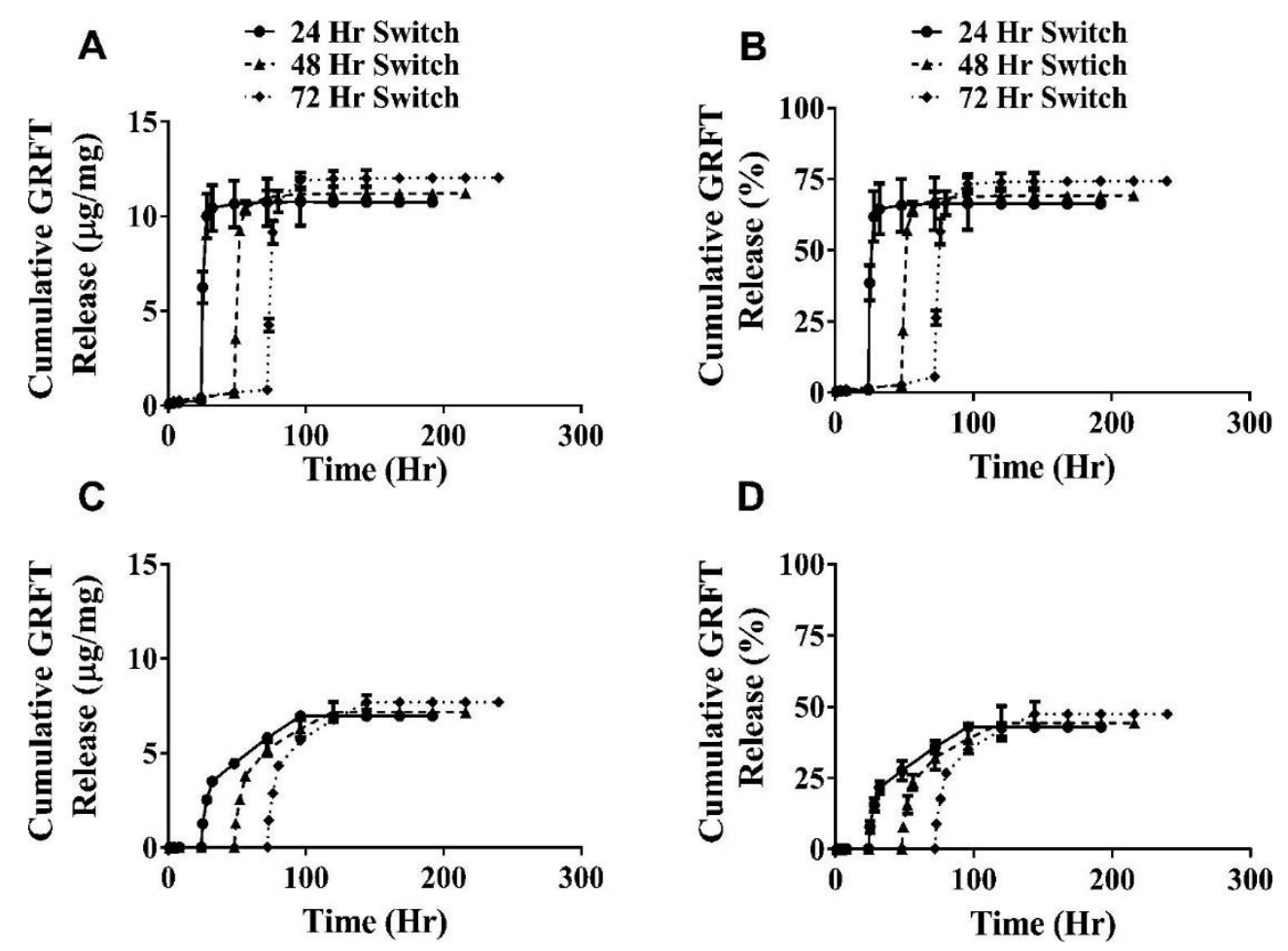

Figure 3.6 In vitro pH-responsive release profiles of GRFT from mPEGPLGA:PBA-co-PAA (90:10, w/w) fibers. The cumulative release of GRFT is shown as a function of mass released $(A, C)$ and percent total loading $(B, D)$. Fibers were incubated in SVF for 24,48 , or $72 \mathrm{~h}$. The media was subsequently "switched" to (A, B) PBS or (C, D) SVF:SSF (1:3) mixture, and fibers were incubated for an additional $72 \mathrm{~h}$. Release values are expressed as the mean \pm SD of triplicate samples. 


\section{HIV-1 Inhibition Studies}

Based on the release results, mPEG-PLGA:PBA-co-PAA (90:10) GRFT fibers were evaluated to provide $\mathrm{pH}$-dependent protection against HIV-1 infection in vitro. First, the activity of GRFT, extracted from electrospun fibers, was assessed to determine whether GRFT is inactivated during electrospinning. Previous studies have shown that harsh solvents and electric field used during the electrospinning process may denature protein and decrease biologic activity ${ }^{298,299}$. Therefore, the inhibitory potential of extracted, relative to free GRFT, was tested against HIV-1 infection. Similar antiviral activity was observed between free GRFT and GRFT extracted from mPEG-PLGA:PBA-co-PAA (90:10) fibers (Figure 3.7). Complete protection against HIV-1 infection was achieved from undiluted fiber extracts (IC 50 $10.6 \mathrm{ng} / \mathrm{mL})$ and free GRFT $(15.5 \mathrm{ng} / \mathrm{mL})$, with a dose-dependent decrease in protection observed for both fiber extract and free GRFT dilutions. 

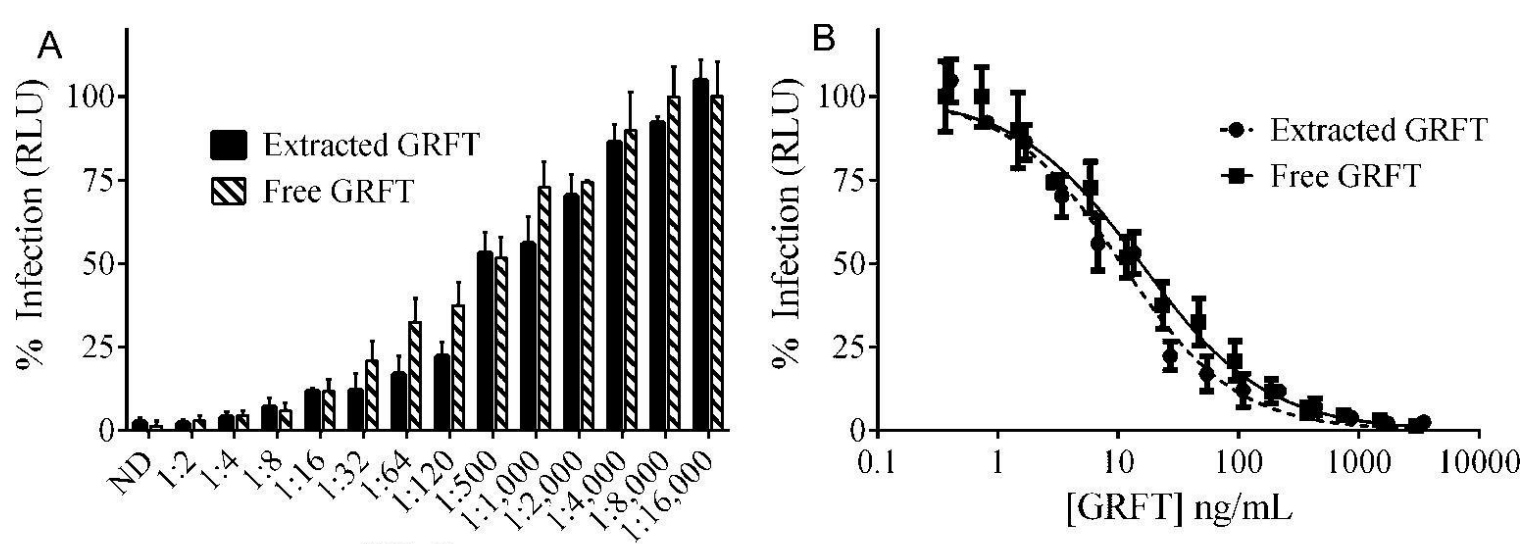

Dilution

Figure 3.7 HIV-1 inhibition assays were conducted to assess the functional activity of extracted GRFT after electrospinning into fibers, relative to free GRFT. GRFT extracted from mPEG-PLGA:PBA-co-PAA (90:10) fibers demonstrates complete efficacy against HIV-1 infection, and similar efficacy to free GRFT. The percent of HIV-1 infected cells, relative to untreated cells, is shown as the mean \pm SD of triplicate samples, as a function of (A) eluate dilution or (B) GRFT concentration. 
To assess the inhibitory activity of GRFT fibers after an induced pH change, mPEG-PLGA:PBA-co-PAA (90:10) fibers were incubated in SVF for either 24, 48, or $72 \mathrm{~h}$, followed by a switch to PBS or SVF:SSF, for an additional hour. After $1 \mathrm{~h}$, the eluates were collected and assessed in HIV-1 inhibition assays. As seen in Figure 3.8A and B, despite different initial incubation periods in SVF $(24,48$, or 72 h), GRFT maintained bioactivity and completely inhibited HIV-1 infection after exposure to PBS. The IC $\mathrm{C}_{50}$ for the $1 \mathrm{~h}$ PBS release eluates, after an initial 24, 48, and $72 \mathrm{~h}$ in SVF were $28.3,29.6$ and, $23.5 \mathrm{ng} / \mathrm{mL}$ respectively. Similarly, complete inhibition of HIV-1 infection was achieved from mPEG-PLGA:PBA-co-PAA fibers that were switched to a more physiologically relevant environment of SVF:SSF after the same durations $(24,48,72 \mathrm{~h})$ (Figure 4.8C and D). The IC 50 values from $1 \mathrm{~h}$ post-switch SVF:SSF release eluates, were 23.2, 23.7 , and $21.3 \mathrm{ng} / \mathrm{mL}$ respectively. The $\mathrm{IC}_{50}$ s of both PBS and SVF:SSF $1 \mathrm{~h}$ post-switch release eluates were not statistically different, demonstrating that GRFT maintains bioactivity regardless of incubation time. 

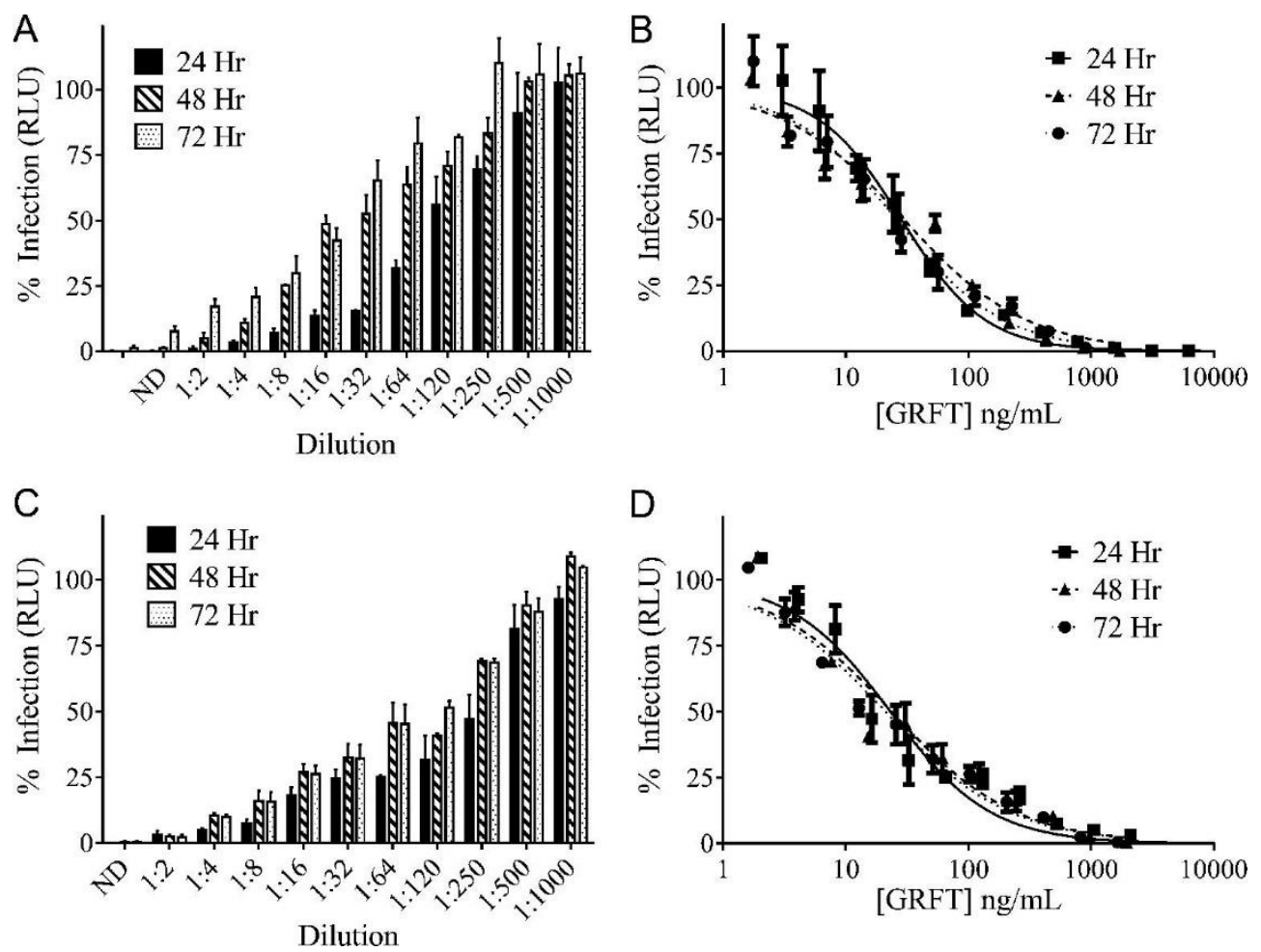

Figure $3.8 \mathrm{HIV}-1$ inhibition assays were conducted to assess the antiviral activity of GRFT mPEG-PLGA:PBA-co-PAA (90:10) fiber release eluates against HIV-1 infection. GRFT mPEG-PLGA:PBA-co-PAA fibers were incubated in SVF for 24, 48, or 72 h. (A) After each time point, SVF was "switched" to PBS, and serial dilutions of fiber eluates were collected to assess antiviral efficacy after $1 \mathrm{~h}$ in PBS. (B) The percent of HIV-1 infected cells, relative to untreated cells, is shown as the mean \pm SD of triplicate samples. (C and D) The study was repeated with fibers "switched" from SVF to SVF:SSF (1:3). The serial dilutions of GRFT release eluates were evaluated against $\mathrm{HIV}-1$ and $\mathrm{IC}_{50}$ values were calculated. 


\section{In Vitro Safety}

To determine the biocompatibility of pH-sensitive GRFT EFs, vaginal epithelial cell lines: VK2, Ect1, and End1 E6/E7 were incubated with 1 and $10 \mathrm{mg} / \mathrm{mL}$ fibers in vitro. As shown in Figure 3.9, all cell lines demonstrated greater than $95 \%$ cell viability after 24,48 , and $72 \mathrm{~h}$ fiber administration, relative to untreated and DMSOtreated cells. There were no statistically significant differences noted in cell viability, as a function of cell line or administration duration. 

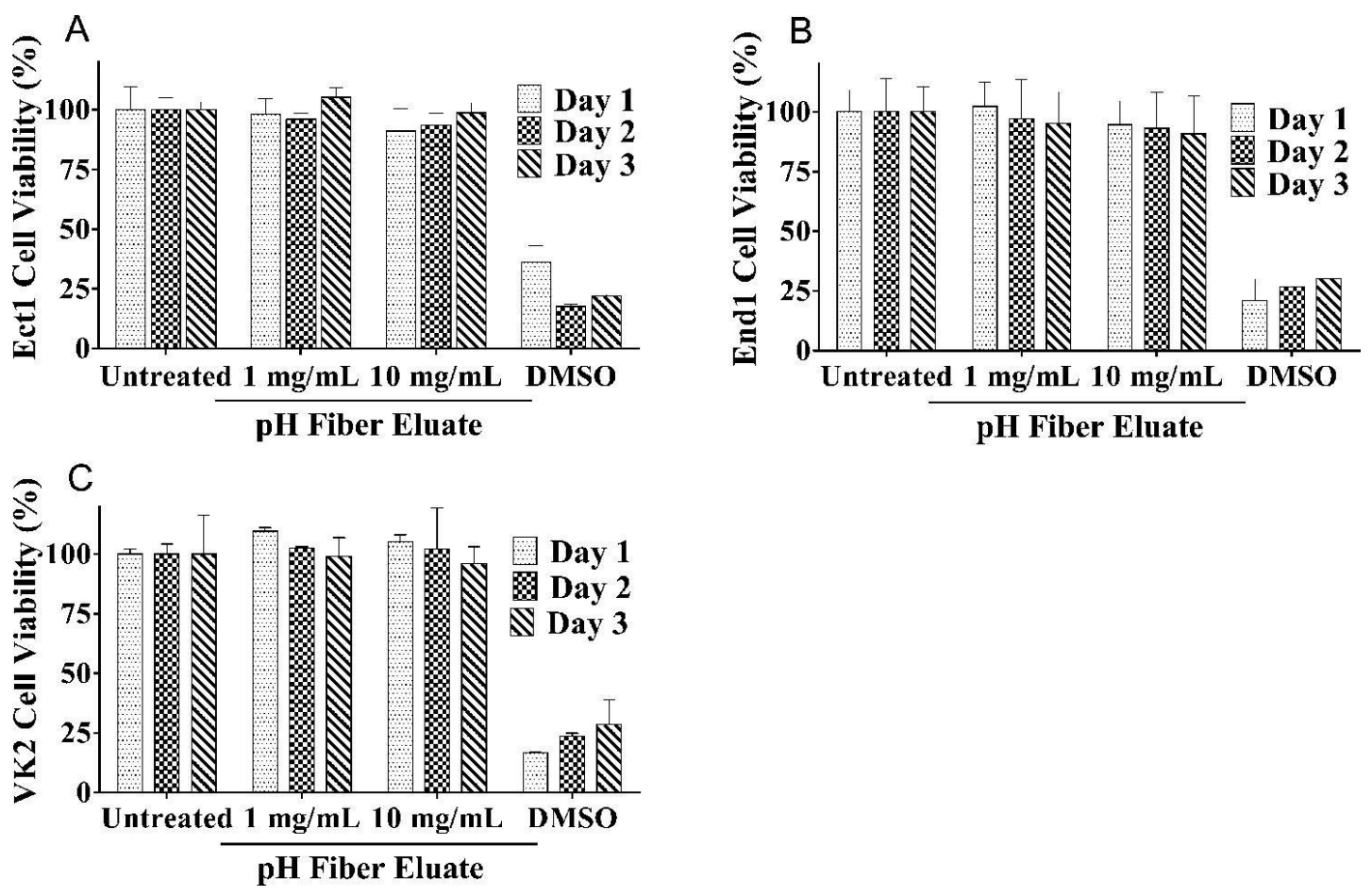

Figure 3.9 In vitro safety evaluation of mPEG-PLGA:PBA-co-PAA (90:10) fibers on cervicovaginal cell viability. (A) Ectocervical (Ect1/E6E7), (B) endocervical (End1/E6E7), (C) and vaginal (VK2/E6E7) epithelial cells were incubated with GRFT mPEG-PLGA:PBA-co-PAA fibers ( 1 and $10 \mathrm{mg} / \mathrm{mL}$ ) for 24,48 , and $72 \mathrm{~h}$ and assessed for cytotoxicity using the MTT assay. All cells had greater than $90 \%$ viability, relative to untreated positive controls. Cell viability is expressed as the mean \pm SD of triplicate samples. 


\section{Discussion}

While previous work has demonstrated the potential to provide $\mathrm{pH}$ responsive release of traditional antivirals, here we present a method to induce the $\mathrm{pH}$-responsive release of the antiviral lectin, GRFT. Griffithsin is a promising new biologic for use against HIV-1, HSV-2, human papillomavirus, and a variety of other viruses, due to its potent binding and antiviral activity $61,62,71,300,301$. Additionally, GRFT has demonstrated stability and safety, prompting its development in clinical trials. As such, we hypothesized that the incorporation of GRFT within an electrospun fiber may provide an effective antiviral delivery vehicle to satisfy the on-demand needs of virus entry inhibition. In this work we demonstrate that these fibers provide $\mathrm{pH}$-inducible release of GRFT while preserving GRFT activity and payload for up to 3 days, prior to exposure to PBS or SVF:SSF solutions.

In our initial studies, fibers comprised of either PLGA or mPEG-PLGA polymers were fabricated to provide sustained-release of GRFT. Based on the biocompatibility of PLGA and its previous utilization in sustained-release applications $^{78}$, it was reasoned that similar sustained-release of GRFT may be achieved. However, GRFT release from PLGA fibers was minimal, and substantially below prophylactic needs, beyond $6 \mathrm{~h}$ application. We hypothesized that the diminished release of GRFT from PLGA fibers may be attributed to the isoelectric point of GRFT ( $\mathrm{pl}=5.4$ ), facilitating GRFT adhesion to, or hydrophobic interactions with, the negatively-charged PLGA polymer. In contrast, GRFT release increased from the slightly more hydrophilic mPEG-PLGA fibers. Despite slightly improved initial release, mPEG-PLGA fibers exhibited sub-optimal release 
at later time points, highlighting the need for an improved formulation that retains GRFT under relevant intravaginal conditions, yet releases GRFT in response to coital cues.

In combination with the challenges of obtaining sustained-release, one of the issues with any sustained-release system, is that cargo is released under temporal conditions that may not prophylactically (or therapeutically) necessitate release. For more expensive and labile biologics, conventional sustained-release platforms (not triggered by stimuli) may be inefficient to deliver active agent, particularly an entry inhibitor like GRFT that should be present in high concentration, to protect against virus entry and exposure. Moreover, from an economic perspective, the premature release of biological molecules that have shorter half-lives or are more expensive to produce may adversely impact overall cost and feasibility. Given these considerations, we sought to develop a vehicle that retains GRFT, and only releases GRFT upon exposure to the more neutral $\mathrm{pH}$ conditions of semen infiltration. We hypothesized that this would be a desirable option, given the role of GRFT as an entry inhibitor.

To test the $\mathrm{pH}$-responsive properties, 100:0, 90:10, 85:15, 80:20, and 72:25 of PLGA:PBA-co-PAA GRFT fibers were evaluated. Increases in the length of hydrophobic alkyl groups on acrylic acid, often result in increases in pKa, affecting the $\mathrm{pH}$ of polymer switch ${ }^{302}$. In addition, pendant acidic groups are typically ionized at a $\mathrm{pH}$ dependent on $\mathrm{pKa}$, in neutral and alkaline solutions, and their induced repulsion affects the physical properties of the polymer. Thus, the ionizable carboxylic acid groups in both PBA and PAA increase solubility at neutral/basic 
$\mathrm{pH}^{288}$. In our studies, it was observed that increased ratios of PBA-co-PAA resulted in increased GRFT release after exposure to PBS and SVF:SSF solutions. We expect this trend is due in part to fiber swelling upon exposure to neutral PBS and SVF:SSF solutions. We additionally observed that even small increases in the ratio of PBA-co-PAA, relative to PLGA, resulted in increased release under both neutral and acidic conditions. In fact, increased GRFT release corresponded with increased ratio of PBA-co-PAA under acidic conditions, which was undesirable for a pH-responsive application. The pKa values of PBA (7.4) and PAA (4.28), indicate that the amount of PBA is driving this release, in both neutral (PBS, SVF:SSF) and acidic (SVF) conditions. Of these selected formulations, the 90:10 blend exhibited the most desirable $\mathrm{pH}$-dependent release profile, and release in PBS and SVF:SSF was improved by utilizing the more hydrophilic mPEG-PLGA fibers.

In addition to the $\mathrm{pH}$-responsive properties imparted by PBA-co-PAA, PLGA provides a biocompatible polymer that lends mechanical stability for use in implants ${ }^{78}$. Moreover, it is well known that PLGA fibers specifically impart high mechanical strength for a variety of applications, such as scaffolds ${ }^{303}$. Given these favorable properties, blending with other polymers can add complementary attributes ${ }^{304}$, here $\mathrm{pH}$-responsive behavior. The anionic polymers PBA and PAA have been applied to a variety of biomedical applications ${ }^{305-311}$. Together, it was anticipated that mPEG-PLGA and PBA-co-PAA may provide a stable mechanical scaffold for administration and longevity in the FRT, while imparting properties that enable prompt dissolution when needed. While not addressed in this immediate work, we seek to assess the mechanical properties in addition to the 
mucoadhesivity often provided by blending with PBA or PAA ${ }^{312}$. For long-term applications, these features would be useful to provide immediate protection with retention and subsequent release of GRFT.

In this work we show that $\mathrm{pH}$-responsive fibers retain GRFT for up to $72 \mathrm{~h}$ in SVF, suggesting that this platform may provide greater active agent stability and tailored release for less frequent administration. In addition, we evaluated GRFT release from fibers exposed to both PBS and SVF:SSF after initial incubation in SVF. Fibers switched to PBS exhibited complete release of GRFT within the first $24 \mathrm{~h}$ (Figure 3.6), whereas fibers incubated in SVF:SSF exhibited a more gradual release, for up to $72 \mathrm{~h}$ after SVF:SSF exposure. This difference in release may be attributed to the increased viscosity and osmolality of simulated seminal fluid relative to $\mathrm{PBS}^{296,313}$. The daily release of GRFT from fibers switched to SVF:SSF was enough to provide protection throughout the $72 \mathrm{~h}$ post-switch incubation period, indicating that these fibers may provide activity for up to 6 days administration in the female reproductive environment. Moreover, previous studies have shown that human semen may be present within the FRT for up to $72 \mathrm{~h}$ postcoitus, during which time multiple exposures to HIV-1 may occur. A platform that provides $\mathrm{pH}$-dependent release coupled with sustained short-term release postswitch may address the administration challenges of short- versus long-term (or unknown) exposure. This platform demonstrates the ability to rapidly release GRFT under $\mathrm{pH}$ conditions of semen exposure, while also preserving GRFT after 3 days in SVF, demonstrating exciting utility relative to traditional delivery platforms. 
In addition to conserving GRFT payload and providing inducible release, mPEG:PBA-co-PAA (90:10) EFs provided corresponding immediate and complete protection against HIV-1 in vitro. Fiber eluates maintained antiviral activity against HIV-1 after different incubation durations, demonstrating their potential utility for administration every few days. Building upon this work, we expect to conduct more prolonged release and efficacy studies in the future. Furthermore, the in vitro release profiles indicate that the fibers retain GRFT for short duration, unlike traditional sustained-release delivery platforms, which often exhibit an initial burst release whether or not it is needed. This design feature may provide enhanced protection relative to the administration of free GRFT alone or other traditional delivery platforms, in which prematurely released GRFT may be shed with mucus, or may be locally diluted when needed. Considering that GRFT acts as an entry inhibitor by interacting with viral envelope proteins, the ability of this $\mathrm{pH}$-responsive platform to provide GRFT release immediately after exposure to SSF, may increase the success rate of viral inhibition by releasing a timely localized concentration of GRFT to inactivate virus.

In addition to relevant release times and efficacy, the design of intravaginal delivery vehicles requires that safety and biocompatibility are considered early in the development process. Particularly for GRFT, which has demonstrated outstanding safety ${ }^{68,69}$, similar safety must be assessed with its integration in new delivery vehicles. In our studies with vaginal epithelial cells, incubation with fiber eluates resulted in greater than $\sim 95 \%$ cell viability after 1,2 , and 3 day exposure, indicating the preliminary safety of these $\mathrm{pH}$-responsive fibers. Future work will 
assess if these fibers induce inflammation or enhance cytokine production in in vivo experiments.

\section{Conclusions}

Drug delivery systems in which active agent release can be tailored to release in response to incoming stimuli are particularly promising for biologics that may lose activity quickly and be expensive to produce. In this study, $\mathrm{pH}$-responsive fibers comprised of PLGA, mPEG-PLGA, and PBA-co-PAA polymer blends were fabricated to provide $\mathrm{pH}$-responsive release of GRFT. Of the formulations tested, the mPEG-PLGA:PBA-Co-PAA $(90: 10)$ blend provided the optimal release of GRFT, exhibiting increased release under more neutral conditions while maintaining minimal release in acidic SVF. In addition, fiber release eluates provided immediate activity against HIV-1 infection while simultaneously retaining GRFT activity. Furthermore, the release profiles demonstrated that fibers provided $\mathrm{pH}$-induced release for at least $72 \mathrm{~h}$, further indicating the utility of this delivery platform to preserve active agent for a minimum of 3-6 days post-administration. 


\section{CHAPTER 4}

\section{RAPID RELEASE GRIFFITHSIN ELECTROSPUN FIBERS FOR USE AGAINST SEXUALLY TRANSMITTED INFECTIONS}

\section{Introduction}

In this study, our goal was to develop rapid-release electrospun fibers that incorporate the biologic GRFT to provide on-demand dual-purpose protection against HIV-1 and HSV-2 infections in vitro, and to demonstrate initial safety and efficacy against HSV-2 infection in vivo. Rapid-release fibers, composed of polyethylene oxide (PEO), polyvinyl alcohol (PVA), and polyvinylpyrrolidone (PVP) were selected due to their established biocompatibility, mucoadhesivity, and rapid dissolution in aqueous environments ${ }^{314-317}$. The ability of GRFT fibers to provide complete protection against both HIV-1 and HSV-2 infections was demonstrated in vitro. Furthermore, the efficacy of GRFT fibers was assessed in a murine model of lethal HSV-2 infection, demonstrating that a single application of PEO, PVA, or PVP GRFT fibers provided potent protection when administered $4 \mathrm{hr}$ prior to infection. In addition, histology and cytokine expression data, assessed from murine reproductive tissues and vaginal lavages, demonstrated the preliminary safety of rapid-release GRFT fibers in vaginal tissue.

\section{Materials and Methods}




\section{Materials.}

PEO (600,000 MW), PVA (87-90\% hydrolyzed, 30,000-70,000 MW), and PVP (1,400,000 MW) were purchased from Sigma Aldrich (St Louis, MO). Organic solvents including dimethyl sulfoxide (DMSO) were also purchased from Sigma Aldrich. Cell culture media and reagents including Dulbecco's modified Eagle medium (DMEM), minimum essential media (MEM), fetal bovine serum (FBS), penicillin, streptomycin, and 4-(2-hydroxyethyl)-1-piperazineethanesulfonic acid (HEPES) were purchased from VWR (Radnor, PA). Keratinocyte Serum Free Media (KSFM) and gentamicin were purchased from Thermo Fisher (Hampton, $\mathrm{NH})$.

\section{Cell Lines and Virus.}

TZM-bl cells, obtained from the National Institutes of Health (NIH) AIDS Research and Reference Reagent Program (ARRRP), were used to assess in vitro HIV-1 infectivity. TZM-bl cells are engineered HeLa cells that express CD4, CCR5 and CXCR4 receptors and contain a Tat-driven luciferase gene, which is activated by HIV-1 infection and permits sensitive and accurate measurements of infection. TZM-bl cells are highly permissive to infection by most strains of HIV-1 and molecularly cloned Env-pseudotyped viruses. TZM-bl cells were cultured in DMEM containing 10\% FBS, $25 \mathrm{mM}$ HEPES, and $50 \mu \mathrm{g} / \mathrm{mL}$ gentamicin.

The Env-pseudotype HIV-1 was produced in house by transducing HEK293T/17 cells with both an envelope (env)-expressing plasmid (CCR5-tropic clade A strain, Q769.h5) and an env-deficient HIV-1 backbone vector (pNL4.3AEnv- 
Luc). Both plasmids were obtained from the NIH AIDS Reagent Program (Cat\# 11884 and 3418). HEK-293T (human embryonic kidney) cells were purchased from ATCC. Cells were maintained in minimum essential medium (MEM) supplemented with $10 \%$ FBS, penicillin $(100 \mu \mathrm{g} / \mathrm{mL})$, and streptomycin (100 $\mu \mathrm{g} / \mathrm{mL})$.

To conduct HSV-2 plaque assays, Vero E6 cells and HSV-2 (4674) were kindly provided by Dr. Kenneth Palmer from the University of Louisville. Cells were maintained in MEM supplemented with $10 \%$ FBS, penicillin $(100 \mu \mathrm{g} / \mathrm{mL})$, and streptomycin $(100 \mu \mathrm{g} / \mathrm{mL})$.

Finally, vaginal keratinocyte (VK2/E6E7), ectocervical (Ect1/E6E7), and endocervical (End1/E6E7) cell lines were used to assess fiber cytotoxicity (all cells courtesy of Dr. Kenneth Palmer, originally from ATCC, Rockville MD). VK2/E6E7 (VK2), Ect1/E6E7 (Ect1), and End1/E6E7 (End1) are well-characterized immortalized cell lines derived from normal human vaginal, ectocervical, and endocervical epithelia, respectively, which are representative of the cell types found within the FRT. Cells were maintained in KSFM supplemented with recombinant human epidermal growth factor $(0.1 \mathrm{ng} / \mathrm{mL})$, bovine pituitary extract (50 $\mu \mathrm{g} / \mathrm{mL})$, calcium chloride $(0.4 \mathrm{mM})$, and $1 \%$ penicillin and streptomycin (100 $\mu g / m L$ each). During cell trypsinization, plating, and counting, cells were neutralized with 1:1 DMEM:KSFM (with 10\% FBS, and 1\% penicillin/streptomycin $(100 \mu \mathrm{g} / \mathrm{mL}$ each)).

\section{Rapid-Release Fiber Fabrication.}


Hydrophilic polymer solutions were fabricated by first weighing polymer into a glass scintillation vial and incubating overnight in $1 \mathrm{~mL}$ of Milli-Q water. To create PVA and PVP fibers with well-defined morphologies, $200 \mathrm{mg}$ of either PVA or PVP were added to $1 \mathrm{~mL}$ Milli-Q water ( $20 \% \mathrm{w} / \mathrm{v}$ solution), while PEO fibers were fabricated by adding $50 \mathrm{mg}$ of polymer to $1 \mathrm{~mL}$ Milli-Q water ( $5 \% \mathrm{w} / \mathrm{v})$. Blank fibers were electrospun with a mandrel-to-syringe distance of $20 \mathrm{~cm}$, flow rate of 0.2 to $0.3 \mathrm{~mL}$, and a voltage of $15 \mathrm{kV}$. The flow rate and voltage were changed to 0.2 $\mathrm{mL} / \mathrm{hr}$ and $25 \mathrm{kV}$ for 1 and $10 \% \mathrm{w} / \mathrm{w}$ (GRFT to polymer weight ratio) GRFT fibers.

\section{Fiber Morphology.}

The morphology of blank PEO, PVA, and PVP fibers, as well as $1 \%$ and 10\% GRFT w/w PEO, PVA, and PVP fibers was assessed using scanning electron microscopy (SEM). After electrospinning, fibers were dried for $24 \mathrm{hr}$ in a desiccator, cut into $5 \mathrm{~mm}$ pieces and placed on double-sided adhesive carbon tabs (Ted Pella Inc., Redding, CA), which were adhered to aluminum stubs. The adhered fiber pieces were sputter coated with a thin gold alloy film using a Bio-Rad E5100 sputter coat system (Hercules, CA). The coating process was operated for $90 \mathrm{~s}$ at $20 \mathrm{~mA}$. Multiple SEM images were acquired using a Supra 35 SEM (Zeiss, Oberkochen, Germany), with images captured under an accelerating voltage of $8 \mathrm{kV}$ and using an average magnification of 1,000 to $5,000 x$. The average fiber diameter was determined with ImageJ software $(\mathrm{NIH}$, Bethesda, $\mathrm{MD})$, and a minimum of 50 fibers were assessed per image.

\section{Fiber Characterization.}


To assess GRFT loading, PEO, PVA, and PVP fibers were weighed ( 3 to 5 $\mathrm{mg}$ ) into separate $1.5 \mathrm{~mL}$ Eppendorf tubes, followed by the addition of $1 \mathrm{~mL}$ PBS. After 1 to $2 \mathrm{~min}$, the dissolved fiber solutions were vortexed and analyzed using ELISA to quantify GRFT loading and encapsulation efficiency (defined as: [actual loading $\div$ theoretical loading] $\times 100$ )

The ELISA was conducted using 96-well Nunc Maxisorp plates as previously described ${ }^{162}$. Briefly, plates were first prepared by coating wells with $100 \mu \mathrm{L}$ of $\mathrm{gp} 120(250 \mathrm{ng} / \mathrm{mL})$ in PBS, and incubating overnight at $4^{\circ} \mathrm{C}$. Afterward, the coating buffer was removed and $300 \mu \mathrm{L}$ of blocking buffer, consisting of PBS with $0.05 \%(\mathrm{v} / \mathrm{v})$ Tween-20 (PBST) and 3\% (w/v) bovine serum albumin, was added to each well. Plates were incubated at room temperature for $2 \mathrm{hr}$ and then washed three times with PBST using a Multiwash III plate washer (Gardner Denver, Milwaukee, WI). One hundred microliters of GRFT standards (ranging from 0.2 to $120 \mathrm{ng} / \mathrm{mL}$ ) and loading extracts were added to each well and incubated for $1 \mathrm{hr}$ at $37^{\circ} \mathrm{C}$. Dilutions of goat anti-GRFT primary antibody $(1: 10,000$, provided by Dr. Kenneth Palmer, University of Louisville) and rabbit anti-goat IgG-HRP secondary antibody (1:20,000, Sigma-Aldrich) were added in volumes of $100 \mu \mathrm{L}$ and each incubated for $1 \mathrm{hr}$ at $37^{\circ} \mathrm{C}$ to detect bound GRFT. Finally, $100 \mu \mathrm{L}$ of $\mathrm{KPL}$ SureBlue TMB microwell peroxidase substrate (Sera Care, Milford, MA, USA) was added to each well for $90 \mathrm{~s}$, and the reaction was quenched with the addition of $100 \mu \mathrm{L}$ of $1 \mathrm{~N} \mathrm{H}_{2} \mathrm{SO}_{4}$ (Thermo Fisher, Waltham, MA). Plate absorbance was measured at $450 \mathrm{~nm}$ on a Synergy HT reader (BioTek, Winooski, VT, USA). Data were analyzed using Prism (GraphPad Software Version 6.0, La Jolla, CA). 


\section{In Vitro HIV-1 Pseudovirus Inhibition Assay.}

The antiviral activity of GRFT fibers against HIV-1 was measured using an in vitro HIV-1 pseudovirus inhibition assay. As previously described ${ }^{75,161,162}, \mathrm{HIV}-1$ inhibition was determined as a function of reduction in luciferase reporter gene expression after a single round of infection in TZM-bl cells. The optimal virus dilution was established prior to the experiments to yield $\sim 100,000$ relative luminescence units (RLUs).

Briefly, $1 \%$ GRFT fibers ( 3 to $5 \mathrm{mg}$ ) were first dissolved in sterile PBS, followed by serial dilutions (1:2) with DMEM to a final volume of $50 \mu \mathrm{L}$ within a 96well plate. TZM-bl cells $(10,000$ cells in $100 \mu \mathrm{L}$ DMEM medium with $10 \mu \mathrm{g} / \mathrm{mL}$ DEAE-dextran) were subsequently added to each well, followed by the addition of $50 \mu \mathrm{L}$ of diluted HIV-1 pseudovirus. Cells were then incubated at $37^{\circ} \mathrm{C}$ for $48 \mathrm{hr}$. Dilutions of free GRFT (stock concentration $50 \mu \mathrm{g} / \mathrm{mL}$ ) ranging from $15 \mathrm{pg} / \mathrm{mL}$ to $120 \mathrm{ng} / \mathrm{mL}$ were similarly prepared for comparison.

After $48 \mathrm{hr}, 100 \mu \mathrm{L}$ culture medium was carefully removed from each well. Luminescence was measured using the Bright-Glo luciferase assay system (Promega Corporation, Madison, WI) by adding $100 \mu \mathrm{L}$ Bright-Glo reagent solution to each well for $5 \mathrm{~min}$. Plates were read via luminescence by the Synergy HT reader (BioTek). All RLU values were corrected by subtracting the RLU of untreated/uninfected cells from the sample RLUs (treated/infected cells). The percent virus inhibition was determined by normalizing the corrected RLUs of treated/infected cells to corrected untreated/infected cells: $\%$ Infection $=[($ sample 
RLU - untreated/uninfected cells) $\div$ (untreated/infected cells untreated/uninfected cells)] $\times 100$. The antiviral activity of GRFT fibers is reported as the half maximal inhibitory concentration $\left(\mathrm{IC}_{50}\right)$, which was calculated by comparing the untreated/infected corrected control RLUs to the corrected RLU values of sample dilutions.

\section{In Vitro HSV-2 Plaque Assay.}

HSV-2 plaque assays were conducted to evaluate the in vitro efficacy of $10 \% \mathrm{w} / \mathrm{w}$ GRFT fibers against HSV-2 infection as previously described ${ }^{161}$. Briefly, Vero E6 cells were seeded at 600,000 cells/well in a 6 -well flat bottom plate (50\% confluence) and grown for $24 \mathrm{hr}$ to confluence. Prior to cell infection, $10 \% \mathrm{w} / \mathrm{w}$ GRFT fibers (30 mg) were dissolved in $20 \mathrm{~mL}$ complete plating media (1\% FBS MEM). Once the cells were fully confluent, the growth media was removed and replaced with $2 \mathrm{~mL}$ of GRFT fiber eluate dilutions, followed by HSV-2 infection (3,000 PFU per well) $1 \mathrm{hr}$ later. Free GRFT $(2,000 \mu \mathrm{g} / \mathrm{mL})$, corresponding to the concentration necessary to provide complete HSV-2 inhibition, was used as a positive control for inhibition, in addition to untreated/uninfected cells. Untreated/infected cells, were used as a negative control of inhibition.

Subsequent to HSV-2 infection, plates were incubated for $48 \mathrm{hr}$ at $37^{\circ} \mathrm{C}$, all media was removed, and cells were fixed with $1.5 \mathrm{~mL}$ methanol for $10 \mathrm{~min}$. Afterward, $0.1 \%$ crystal violet was applied for $30 \mathrm{~min}$ to stain the plaques. Finally, plates were washed with Milli-Q water, and plaques were counted after drying. Plaque numbers from experimental groups were normalized relative to the number 
of plaques in untreated/infected cells ( 280-300 plaques per well). Samples were analyzed in triplicate, and GraphPad Prism software was used to determine and compare the $\mathrm{IC}_{50}$ values of GRFT fibers to free GRFT.

\section{In Vitro Cytotoxicity.}

Vaginal epithelial (VK2/E6E7), ectocervical (Ect1/E6E7), and endocervical (End1/E6E7) cells were administered either blank or GRFT fibers to assess in vitro safety. Cells were plated at a density of 50,000 cells/well in 96 -well plates and incubated in triplicate with $0.5 \mathrm{mg}$ fiber pieces placed in the solution $(2.5 \mathrm{mg} / \mathrm{mL}$ final concentration). No treatment (media alone) and 10\% DMSO were used as positive and negative controls of cell viability, respectively. After 24,48 , and $72 \mathrm{hr}$, $20 \mu \mathrm{L}$ of MTT reagent was added to the cells and incubated for an additional $4 \mathrm{hr}$, followed by overnight lysis with the addition of $100 \mu \mathrm{L}$ lysis buffer (10\% sodium dodecyl sulfate in $0.01 \mathrm{M}$ hydrochloric acid). Absorbance measurements $(570 \mathrm{~nm})$ were taken the following day. All sample absorbance values were normalized to untreated cell absorbance to obtain percent viability.

\section{In Vivo Efficacy against a Lethal Dose of HSV-2 Infection.}

All in vivo experimental procedures were approved by the University of Louisville's Institutional Animal Care and Use Committee (IACUC 17135) prior to testing. All animal studies were conducted using 5-week-old female BALB/c mice (Jackson Laboratory, Bar Harbor, ME) to evaluate the efficacy and safety of GRFT fibers. For efficacy studies, mice were administered either blank or GRFT fibers ( 5 $\mathrm{mg}$ ) that were UV-sterilized for $15 \mathrm{~s}$. Mice were subcutaneously injected with $3 \mathrm{mg}$ 
Depo-Provera (Revive, Madison, $\mathrm{NJ}$ ) to induce the diestrous stage of their cycle, 5 days prior to fiber administration.

To determine the efficacy of GRFT fibers against HSV-2 infection, mice were administered a single dose of either GRFT PEO, PVA, or PVP fibers, or control groups $(n=20)$. Twenty-four hours after fiber administration, mice were challenged with HSV-2 (20 $\mu \mathrm{L}, \mathrm{LD}_{90}$ 5,000 PFU). Untreated/infected mice were used as positive controls for infection, while untreated/uninfected mice and infected mice treated with free GRFT (20 $\mu \mathrm{L}$ of $1,000 \mu \mathrm{g} / \mathrm{mL}, 20 \mu \mathrm{g}$ GRFT) served as negative controls. Free GRFT doses were based on previous studies with GRFT gels that were shown to provide in vivo protection against HSV-2 infection ${ }^{65}$. Blank PEO fibers were administered as an additional control group in efficacy studies. Mice were monitored daily for 14 days after HSV-2 challenge using an

established 4-point scale to monitor the progression of viral infection ${ }^{69,318}$. Each day, mice were weighed and examined for signs of neurological and epithelial damage. After the two-week period following HSV-2 challenge, mice were euthanized and Kaplan-Meier survival curves were generated. Log-ranked post hoc tests were conducted to assess the statistical significance between groups.

\section{In Vivo Safety.}

To assess the safety of fiber administration, mice were similarly subcutaneously injected with $3 \mathrm{mg}$ of Depo-Provera, 5 days prior to fiber administration. Afterward, UV-sterilized blank fibers $(5 \mathrm{mg})$ were intravaginally administered to mice under isoflurane anesthesia using sterile tweezers. 
Treatment groups included mice administered blank PEO, PVA, or PVP fibers, while control groups included untreated mice, or mice treated with $20 \mu \mathrm{L}$ free GRFT in PBS $(1,000 \mu \mathrm{g} / \mathrm{mL})$ or $40 \mu \mathrm{L}$ of Conceptrol gel. An additional sham control was used to mimic fiber administration using tweezers alone. Mice were euthanized 24 or $72 \mathrm{hr}$ after fiber administration and mouse reproductive tracts and vaginal lavages were collected and stored at $-80^{\circ} \mathrm{C}$ following euthanasia $(n=3)$.

The structural integrity of collected reproductive tracts was evaluated using histological analysis. First, tissue samples were washed with PBS, followed by fixation with $4 \%$ paraformaldehyde. Samples were then embedded in a paraffin block, and stained with hematoxylin and eosin (H\&E). Sample cross-sections were analyzed by a pathologist blinded to the treatment groups.

To determine cytokine levels after blank fiber administration, murine reproductive tracts and vaginal lavages were assessed using a Luminex assay. First, $20 \mu \mathrm{L}$ of T-Per solution (Thermo Fisher) containing 1\% Halt Protease Inhibitor Cocktail (Thermo Fisher) was added per milligram of reproductive tissue. Approximately 20 zirconia/silica beads (BioSpec Productions) were added to each sample, followed by homogenization at $4,500 x$ for $180 \mathrm{~s}$ using Precellys 24 homogenizer instrument (Bertin, France). Homogenized samples were cooled on ice for $5 \mathrm{~min}$, and centrifuged at $10,000 \times \mathrm{g}$ at $4^{\circ} \mathrm{C}$ for $5 \mathrm{~min}$. Afterward, sample supernatants were collected, aliquoted, and stored at $-80^{\circ} \mathrm{C}$ for further study. Prior to conducting the Luminex assay, interleukin-1 beta (IL-1 $1 \beta)$ levels were tested in reproductive tissue samples using specific ELISA Ready-SET-Go! kits (Thermo Fisher). Finally, Luminex assays were used to quantify the cytokine levels in 
collected mouse tissue and lavages. Cytokines including G-CSF, IFN-y, IL-1a, IL$1 \beta$, IL-2, IL-6, IP-10, MCP-1, MIP-1 $\alpha$, MIP1- $\beta$, MIP-2, and TNF- $\alpha$ were selected based on previous studies that examined these markers for intravaginal inflammation and damage ${ }^{65,69}$.

\section{Statistical Analysis.}

Unless otherwise noted, all in vitro experiments were conducted in triplicate, with a minimum of 3 replicates per sample. Statistical analysis of samples assessing fiber morphology, fiber characterization, in vitro assays, and in vivo safety studies was performed by using one-way ANOVA with the Bonferroni post hoc test $(p<0.05)$. For murine studies assessing viral efficacy, log-ranked post hoc tests were conducted to assess statistical significance as a function of treatment group and survival outcome.

\section{Results}

\section{Fiber Size and Morphology.}

The morphology of blank and GRFT PEO, PVA, and PVP fibers is shown in Figure 4.1. All fibers demonstrated well-rounded fiber morphology with average diameters ranging from 220 to $507 \mathrm{~nm}$ (Supplemental Table 4.1). The addition of $1 \%$ GRFT w/w to PEO and PVP fibers resulted in significantly decreased diameters of 239 and $242 \mathrm{~nm}$, while no statistical significance was observed between blank PVA and 1\% GRFT PVA fibers that shared similar diameters regardless of GRFT incorporation. The addition of 10\% w/w GRFT produced fibers with diameters spanning 243 to $339 \mathrm{~nm}$, demonstrating a statistically significant 
increase in fiber diameter for $10 \% \mathrm{w} / \mathrm{w}$ GRFT PVA and PVP fibers relative to $1 \%$ w/w GRFT PVA and PVP fibers. However, PEO fiber diameters remained unchanged with additional GRFT incorporation. Within similarly loaded GRFT fibers, statistical significance in fiber diameter was observed between the $10 \%$ GRFT PEO and PVA fibers, while no statistical significance was observed across the $1 \% \mathrm{w} / \mathrm{w}$ GRFT formulations. 

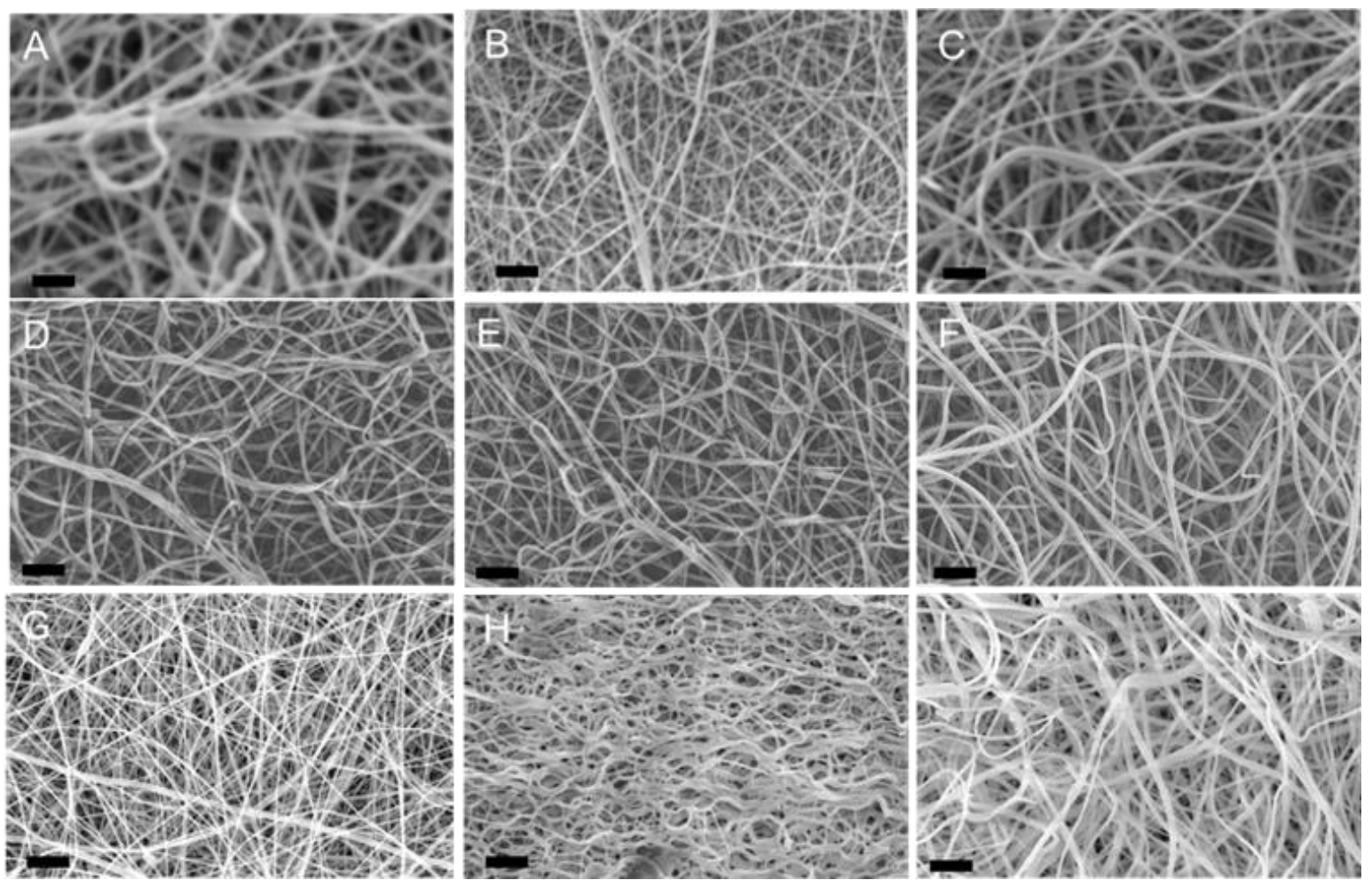

Figure 4.1. Scanning electron microscopy (SEM) images of (A-C) blank, (D-F) $1 \%$ w/w GRFT, and (G-I) $10 \%$ w/w GRFT fibers. (A) $5 \%$ PEO, (B) 20\% PVA, (C) 20\% PVP blank fibers; (D) 5\% PEO, (E) 20\% PVA, (F) 20\% PVP fibers incorporating 1\% w/w GRFT; and (G) 5\% PEO, (H) 20\% PVA, (I) 20\% PVP fibers incorporating 10\% w/w GRFT. Scale bars represent $2 \mu \mathrm{m}$. 
Supplemental Table 4.1. Fiber diameters as a function of polymer formulation and GRFT content. Statistical significance between different polymers with the same GRFT loading is shown as *, while statistical significance between the same polymer type with different GRFT loading is shown as ${ }^{\#}(p<0.05)$. Statistical significance was assessed using one-way ANOVA with the Bonferroni post hoc test.

\begin{tabular}{|c|c|}
\hline Fiber Formulation & Diame ter $(\mathbf{n m})$ \\
\hline Blank PEO & $507 \pm 147$ \\
\hline Blank PVA & $249 \pm 84^{*}$ \\
\hline Blank PVP & $418 \pm 137^{\#}$ \\
\hline PEO 1\% GRFT & $239 \pm 53^{\#}$ \\
\hline PVA 1\% GRFT & $220 \pm 59$ \\
\hline PVP 1\% GRFT & $242 \pm 57$ \\
\hline PEO 10\% GRFT & $243 \pm 95^{*}$ \\
\hline PVA 10\% GRFT & $339 \pm 99^{\#}$ \\
\hline PVP 10\% GRFT & $324 \pm 79^{\#}$ \\
\hline
\end{tabular}




\section{Fiber Characterization.}

GRFT loading was assessed using an ELISA (Figure 4.2). For $1 \% \mathrm{w} / \mathrm{w}$ GRFT PEO, PVA, and PVP fibers, GRFT loading ranged from 7.4 to $9.7 \mu \mathrm{g}$ GRFT/mg fiber, exhibiting no statistical significance between formulations. For 10\% w/w GRFT PEO, PVA, and PVP fibers, GRFT loading was 84.8, 69.6, and 62.4 $\mu \mathrm{g}$ GRFT/mg fiber, respectively, with PEO fibers demonstrating statistically higher loading than PVP fibers. Correspondingly, the encapsulation efficiencies for each fiber formulation ranged from 74.0 to $97.2 \%$, and 62.4 to $84.2 \%$ for 1 and 10\% GRFT fibers, respectively, demonstrating consistently high GRFT loading and suggesting electrospinning compatibility between the polymer and lectin. There were no observable trends between GRFT encapsulation efficiency and fiber diameters. 

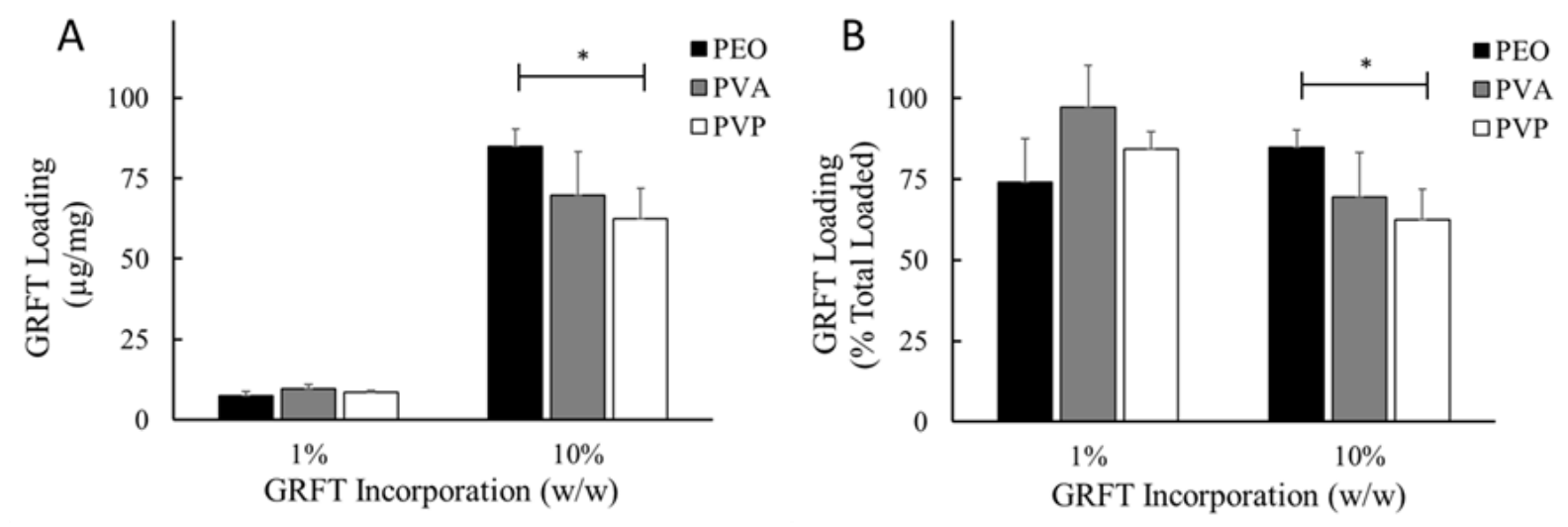

Figure 4.2. GRFT loading in different hydrophilic fiber formulations. Eluates from GRFT fibers dissolved in PBS were used to determine GRFT loading via ELISA. GRFT loading is expressed as the mean \pm standard deviation of triplicate readings of three independent fiber batches. Statistical significance between fiber formulations with the same loading are shown $\left({ }^{*} p<0.05\right)$. 


\section{In Vitro HIV-1 and HSV-2 Inhibition from GRFT Fibers.}

The dual-purpose antiviral activity of GRFT fibers was determined using HIV-1 pseudovirus and HSV-2 plaque inhibition assays. For HIV-1 inhibition studies, all fibers demonstrated complete and dose-dependent HIV-1 inhibition (Figure 4.3A, B). The $\mathrm{IC}_{50}$ values for PEO, PVA, and PVP fibers administered 1 and $24 \mathrm{hr}$ prior to infection ranged from $17.3 \pm 7.2$ to $26.7 \pm 7.7 \mathrm{ng} / \mathrm{mL}$, relative to $24.1 \pm 15.6$ and $22.8 \pm 12.2 \mathrm{ng} / \mathrm{mL}$ for free GRFT at 1 and $24 \mathrm{hr}$ respectively (Table 4.1). No statistical significance was observed between the IC 50 values of GRFT PEO, PVA, and PVP fibers, suggesting no differences in efficacy based on polymer type or as a function of administration time with respect to cell infection. Moreover, similar inhibition values relative to free GRFT indicate that the electrospinning process maintains the functional activity of GRFT. 

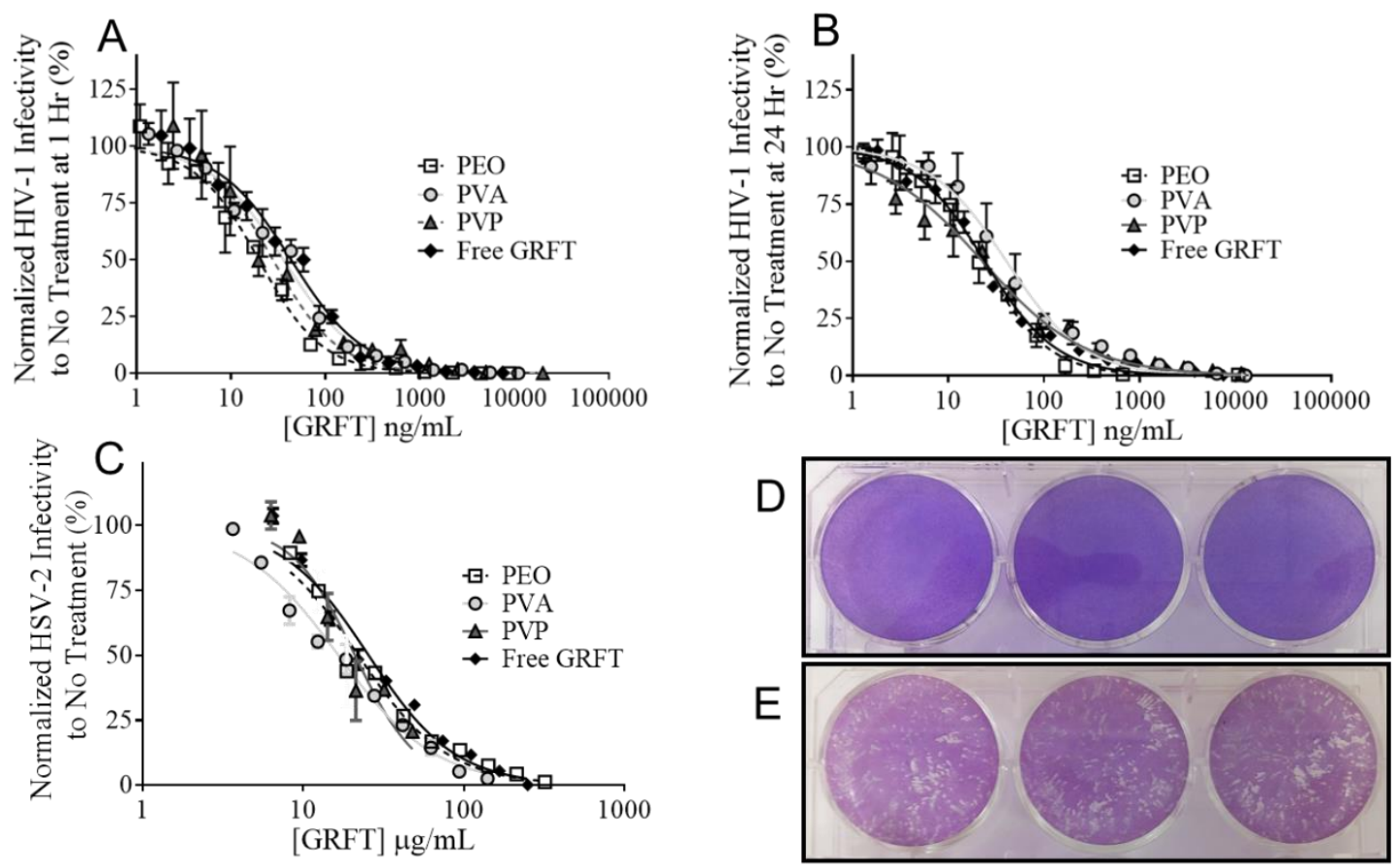

Figure 4.3. GRFT fibers demonstrate complete protection against in vitro HIV-1 and HSV-2 infections. Three independent batches of $1 \% \mathrm{w} / \mathrm{w}$ GRFT fibers were assessed for their ability to inhibit HIV-1 infection. Fiber eluates were incubated with cells (A) $1 \mathrm{hr}$ and (B) $24 \mathrm{hr}$ prior to HIV-1 infection. GRFT released from all three fiber formulations achieved complete efficacy against HIV-1 infection, similar to free GRFT. (C) In vitro HSV-2 plaque assays were performed using $10 \% \mathrm{w} / \mathrm{w}$ GRFT fibers, which similarly achieved complete efficacy against HSV-2 infection in vitro, similar to free GRFT. (D) Wells treated with GRFT fibers showed decreased plaque sizes and numbers relative to (E) untreated (or blank fibertreated, data not shown) cells infected with HSV-2. The percent infection relative to untreated/infected control groups for both HIV-1 and HSV-2 assays is shown as the mean \pm standard deviation of triplicate readings. 
Table 4.1. IC 50 values from in vitro HIV-1 and HSV-2 infectivity assays. GRFT eluates from rapid-release fibers were assessed against HIV-1 and HSV-2 infections, and compared to free GRFT using one-way ANOVA with the Bonferroni post hoc test. Fibers demonstrated comparable activity relative to free GRFT. No statistical significance between groups was observed in HIV-1 inhibition studies as a function of fiber formulation or with respect to administration time. In HSV-2 plaque inhibition assays, GRFT PVA fibers demonstrated lower $\mathrm{IC}_{50}$ values relative to other formulations and free GRFT-treated controls $(p<0.05)$. The average $\mathrm{IC}_{50}$ values are expressed as the mean \pm standard deviation. Statistical significance between GRFT PVA fibers and free GRFT is shown as $\left({ }^{*} p<0.05\right)$.

\begin{tabular}{|c|c|c|c|}
\hline Fiber Formulation & $\mathbf{1} \mathbf{H r} \mathbf{H I V}-\mathbf{1} \mathbf{~ I C}_{\mathbf{5 0}}(\mathbf{n g} / \mathbf{m L})$ & $\mathbf{2 4} \mathbf{H r} \mathbf{H I V}-\mathbf{1} \mathbf{I C}_{\mathbf{5 0}}(\mathbf{n g} / \mathbf{m L})$ & ${\mathbf{1 ~ H r ~ H S V - 2 ~} \mathbf{~ I C}_{\mathbf{5 0}}(\boldsymbol{\mu} \mathbf{g} / \mathbf{m L})}$ \\
\hline PEO & $17.9 \pm 5.4$ & $17.3 \pm 7.2$ & $22.0 \pm 2.1$ \\
\hline PVA & $26.7 \pm 7.7$ & $21.7 \pm 14.8$ & $16.6 \pm 0.9^{*}$ \\
\hline PVP & $26.6 \pm 2.7$ & $23.5 \pm 14.1$ & $21.0 \pm 2.4$ \\
\hline Free GRFT & $24.1 \pm 15.6$ & $22.8 \pm 12.2$ & $25.5 \pm 0.5$ \\
\hline
\end{tabular}


Plaque assays were used to assess the ability of GRFT fibers to inhibit HSV-2 infection. Fibers containing a higher concentration of GRFT $(10 \% \mathrm{w} / \mathrm{w})$ were evaluated, due to the increased concentration of GRFT needed to inhibit HSV-2, relative to HIV-1 infection. GRFT PEO, PVA and PVP fibers demonstrated equivalent protection against HSV-2 infection, relative to free GRFT (IC $5025.5 \pm$ $0.5 \mu \mathrm{g} / \mathrm{mL}$ ), with $\mathrm{IC}_{50} \mathrm{~S}$ of $22.0 \pm 2.14,16.6, \pm 0.92$ and $21.0 \pm 2.4 \mu \mathrm{g} / \mathrm{mL}$ (Figure 4.3C). No statistical significance in efficacy was observed between GRFT fibers and free GRFT, except for PVA fibers, which showed a lower $\mathrm{IC}_{50}$ value relative to free GRFT ( $p<0.05$, Table 4.1). Moreover, administration of all GRFT fiber formulations resulted in decreases in both plaque number and size, relative to untreated/infected controls (Figure 4.3D, E).

\section{In Vitro Cytotoxicity.}

To assess the cytotoxicity of rapid-release fibers, MTT assays were conducted using VK2/, Ect1/, and End1/E6E7 cell lines. All cell lines, incubated with 1 and 10\% w/w GRFT fibers, demonstrated greater than $93 \%$ viability after 24, 48, and $72 \mathrm{hr}$ fiber administration, relative to untreated cells (Figure 4.4). No statistical significance in cell viability was observed as a function of polymer type or GRFT loading. 

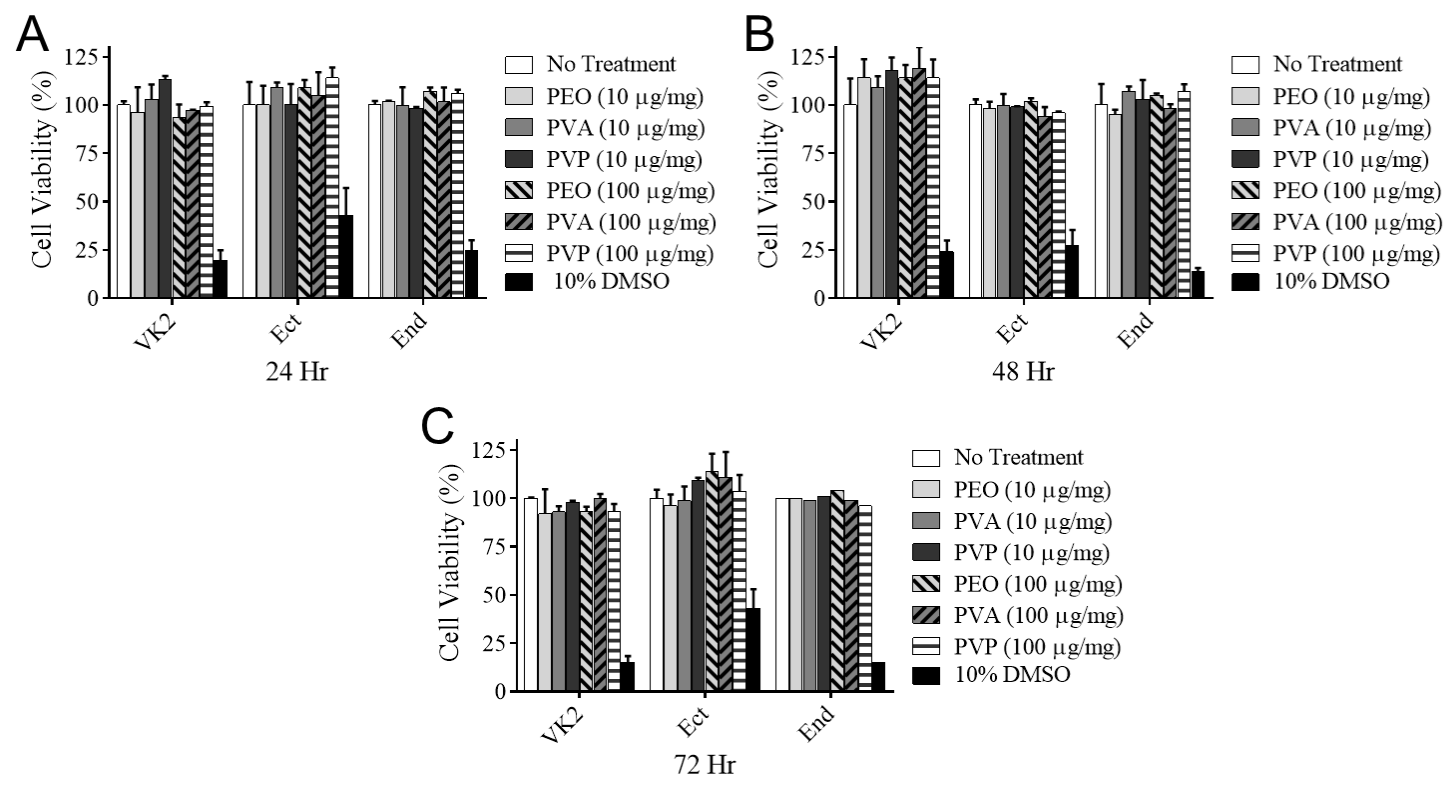

Figure 4.4 The cytotoxicity of PEO, PVA, and PVP fibers administered to vaginal VK2/E6E7, Ect1/E6E7, and End1/E6E7 cells for (A) 24, (B) 48, and (C) $72 \mathrm{hr}$ was assessed using the MTT assay. Greater than $93 \%$ viability was observed across all cell lines for all fiber formulations. 


\section{In Vivo Efficacy against HSV-2 Infection.}

The antiviral efficacy of rapid-release GRFT fibers was assessed in a murine model of lethal HSV-2 infection (Table 4.2). A single dose of $10 \% \mathrm{w} / \mathrm{w}$ GRFT fibers was intravaginally administered to female BALB/c mice, followed by a single HSV-2 challenge with 5,000 PFU (LD90), $4 \mathrm{hr}$ after fiber insertion (Figure 4.5A, B). Mice were evaluated daily for progression of HSV-2 infection for $14 \mathrm{~d}$ post-infection (Figure 4.5C). Mice that were administered GRFT fibers (PEO, PVA, or PVP) exhibited statistically significant decreases in HSV-2 infectivity, with 85, 95 , and $100 \%$ survivability respectively, relative to untreated/infected controls (5\% survivability, $\mathrm{p}<0.05)$. In addition, mice administered GRFT PEO, PVA, or PVP fibers exhibited comparable protection against HSV-2 relative to free GRFT ( $p$ > $0.05)$, while blank PEO fibers imparted protection to $50 \%$ of the animals $(p<0.05)$. 
Table 4.2. GRFT doses administered in fibers or gel during in vivo HSV-2 infectivity studies.

\begin{tabular}{|c|c|c|}
\hline Formulation Name & $\begin{array}{c}\text { Amount of Vehicle } \\
\text { Adminis tered per Mouse }\end{array}$ & $\begin{array}{c}\text { Total GRFT Adminis tered } \\
\text { Based on Loading }(\boldsymbol{\mu} \mathbf{g})\end{array}$ \\
\hline GRFT PEO & $5 \mathrm{mg}$ fiber & 420 \\
\hline GRFT PVA & $5 \mathrm{mg}$ fiber & 350 \\
\hline GRFT PVP & $5 \mathrm{mg}$ fiber & 312 \\
\hline $0.1 \%$ w/v GRFT Solution & $20 \mu \mathrm{L}$ in PBS & 20 \\
\hline
\end{tabular}


A

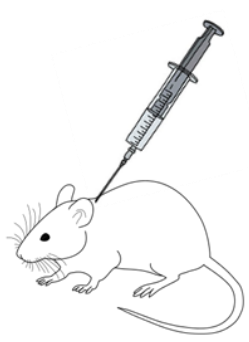

Depo-Provera

Administration

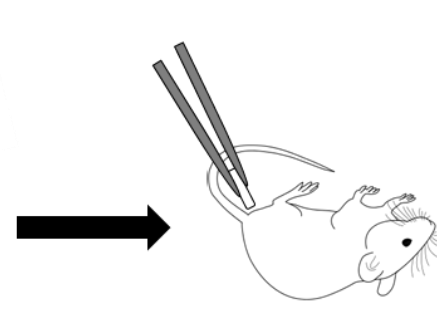

Fiber or Control

Administration

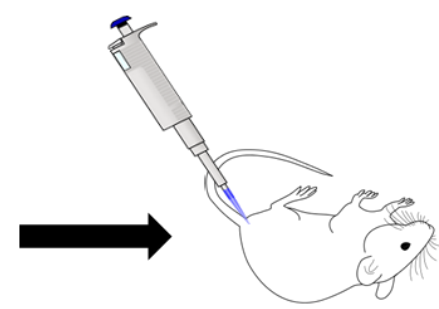

HSV-2 Infection

B

Efficacy Studies
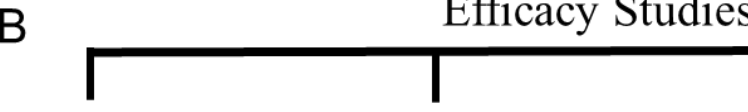

Day -5

Depo-Provera

Administration

Day 0

GRFT Fiber and

Control Administration
(4 Hr)

HSV-2 Infection
Day 14

End of Study

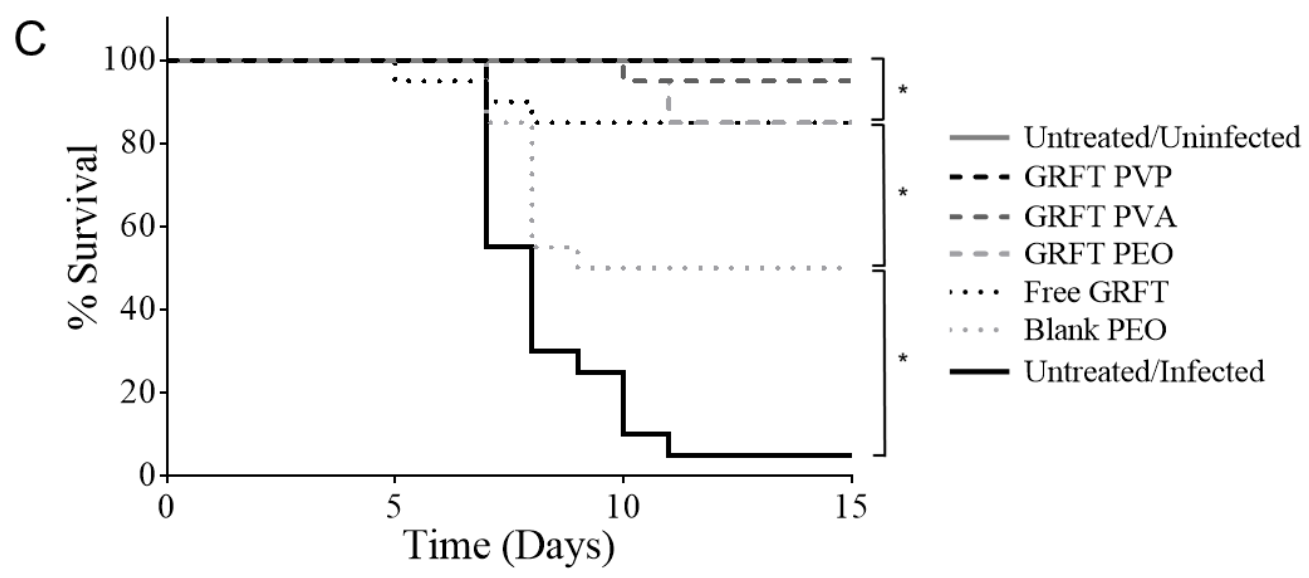

Figure 4.5. Schematic timetable and Kaplan-Meier survival curves of in vivo HSV2 efficacy study. (A) Sequence of murine treatments during the course of the efficacy study and (B) timeline of HSV-2 efficacy study. (C) GRFT fibers (10\% w/w) were assessed for their ability to protect mice against a lethal challenge (LD90) of HSV-2 infection $(n=20)$. All GRFT fiber formulations demonstrated strong protection against HSV-2 infection, resulting in 85 to $100 \%$ murine survivability. Blank fibers also demonstrated partial protection and showed significant survivability $(50 \%)$, relative to the untreated/infected control group. In contrast, 
untreated/infected mice demonstrated only $5 \%$ survivability. Statistical significance between experimental groups is shown as $\left({ }^{*} p<0.05\right)$. 
Another important finding from this study was the difference in infection progression between untreated/infected mice and mice treated with GRFT fibers (Figure 4.6). The first symptoms of HSV-2 infection in mice typically manifest 4 to 5 days post-infection, during which time mice exhibit symptoms of localized swelling in the vaginal area and decreased hind leg mobility. After 5 to 8 days, $\sim 75 \%$ of mice from the untreated/infected control group required euthanasia due to the rapid progression and severity of infection. In contrast, all mice administered GRFT fibers (PEO, PVA, or PVP) or free GRFT that exhibited symptoms, showed decreased progression of infection relative to untreated/infected mice over the same duration. The mice that did not survive infection despite pre-treatment with GRFT fibers or free GRFT (representing up to $15 \%$ total mice, respectively) exhibited a more gradual progression of infection, requiring euthanasia 7 to $10 \mathrm{~d}$, instead of 5 to $8 \mathrm{~d}$ post-infection. The prolonged duration of viral quiescence suggests that GRFT may provide partial protection against infection for the few mice that exhibited overt signs of infection. In comparison, the administration of blank fibers showed no change in progression of HSV-2 infection in mice, relative to untreated/infected controls. Finally, for the few infected mice treated with free GRFT (1 of 20) or GRFT PVA fibers (2 of 20), initial symptoms disappeared near of the end of the study. The decreased levels of infection in combination with the more gradual progression demonstrate the ability of GRFT fibers to protect against a lethal dose of HSV-2 after a single application. 
GRFT PEO

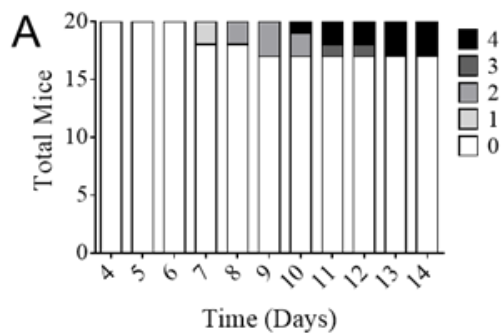

GRFT PVA

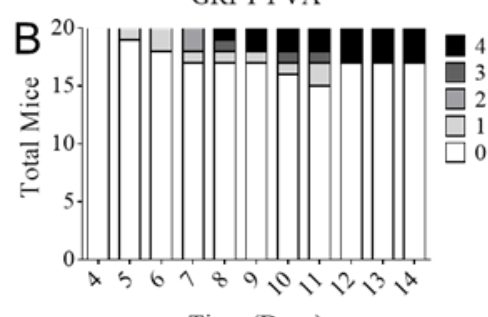

Time (Days)
GRFT PVP

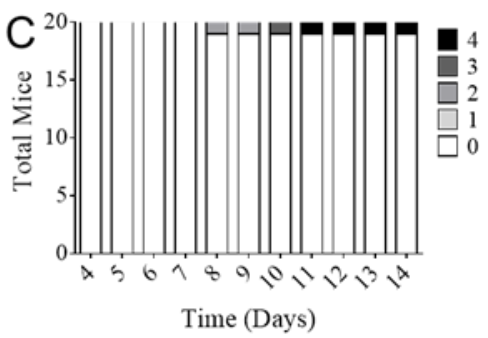

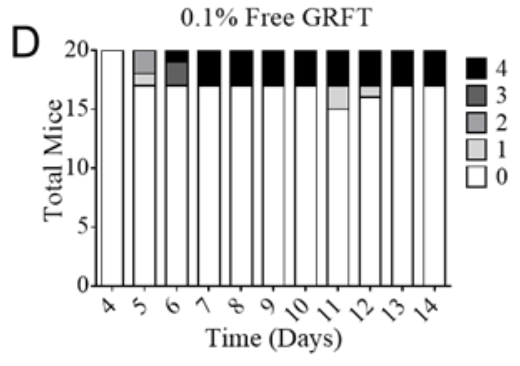

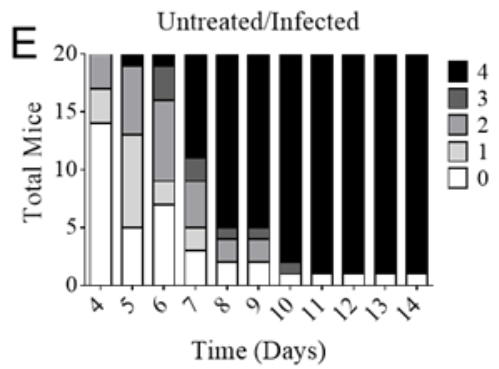

Figure 4.6 Griffithsin fibers protect mice against HSV-2 infection. Mice were administered $10 \% \mathrm{w} / \mathrm{w}$ GRFT fibers $4 \mathrm{hr}$ prior to HSV-2 infection (LD90). Mice were evaluated and scored for progression of infection once daily for $14 \mathrm{~d}$ post-infection. Infected mice were administered (A) PEO, (B) PVA, (C) PVP GRFT fibers, as well as (D) free GRFT, or (E) no treatment. Mice administered GRFT fibers and free GRFT exhibited decreased severity of infection, as well as more gradual progression of infection, relative to untreated/infected animals. 


\section{In Vivo Safety.}

To assess the in vivo safety of rapid-release platforms, fibers were intravaginally administered to mice, and reproductive tissue and vaginal lavages were collected and analyzed 24 and $72 \mathrm{hr}$ following administration.

Tissue samples were evaluated for possible edema of muscle, interstitial, and epithelial tissue. In addition, untreated and blank fiber-treated cervical and vaginal epithelia morphologies were compared, and assessed for possible keratinization and goblet cell presence. A score ranging from 1 to 4 was used to determine the severity of epithelial changes. Figure 4.7 shows images of tissues collected $24 \mathrm{hr}$ after fiber administration. Extracted tissues from untreated controls demonstrated compact squamous and cervical epithelial tissue, with no evidence of edema nor inflammation. Samples from tissues treated with blank PEO, PVA, and PVP fibers showed similar morphology and normality, relative to untreated controls (scores of 1 to 2). Furthermore, there was no increase in lymphocyte accumulation in fiber-treated groups. Overall the tissues from blank fiber-treated mice were comparable to tissues from untreated mice; however, one PVA-treated sample exhibited increased levels of mucin secretion and neutrophil presence (score 3), yet the vaginal and cervical epithelia were intact (Supplemental Figure

4.1A). Results from tissues collected after $72 \mathrm{hr}$ administration were similar to 24 hr samples (images not shown). 


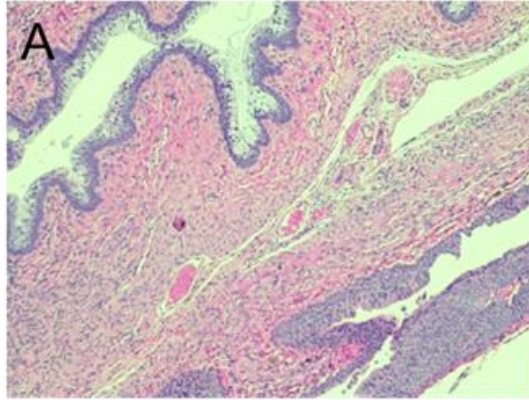

Untreated

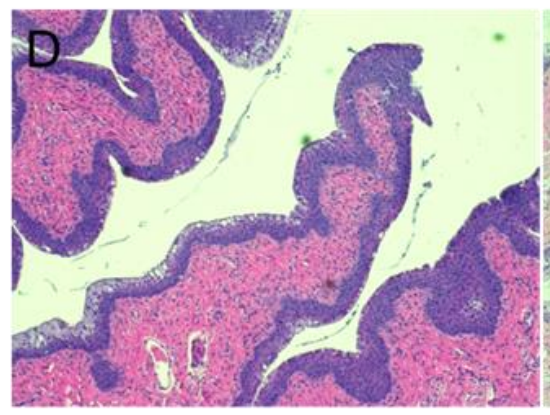

Blank PEO

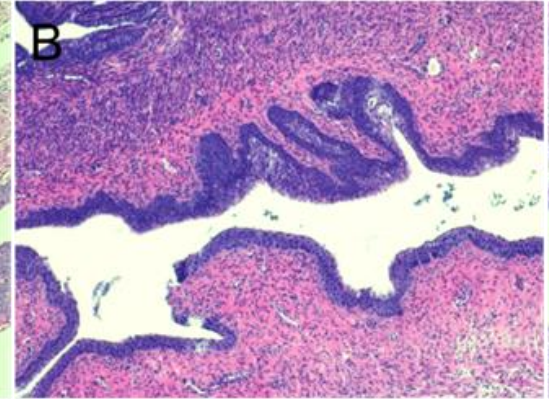

$4 \%$ N-9 Gel

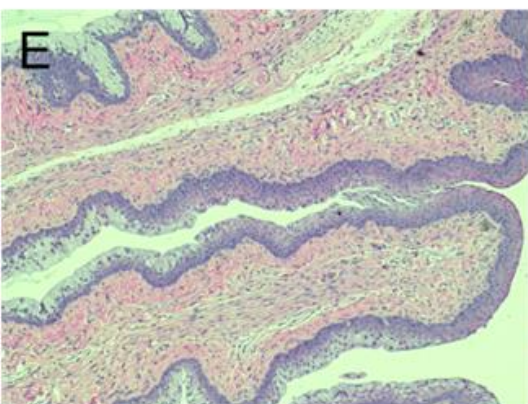

Blank PVA

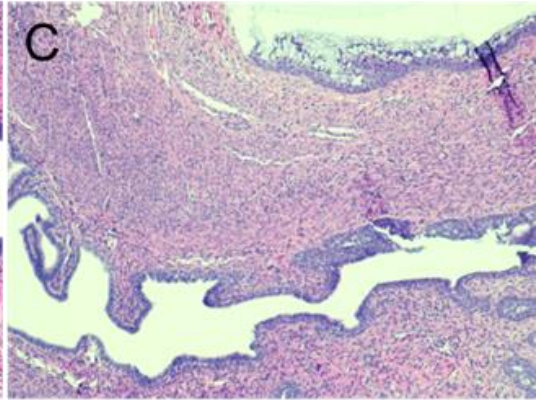

Sham Control

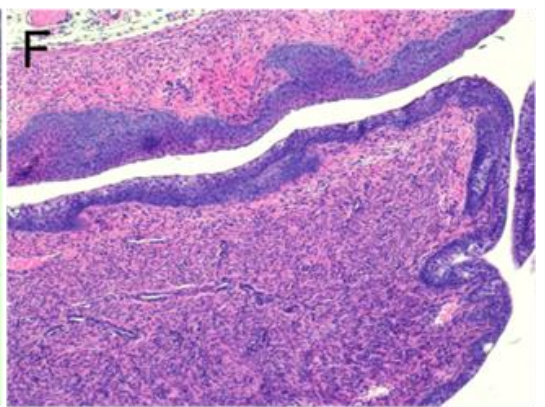

Blank PVP

Figure 4.7. The in vivo safety of rapid-release fibers was assessed by intravaginally administering fibers for 24 and $72 \mathrm{hr}$. Images depict H\&E stained tissues of murine reproductive tracts exposed (A) no treatment, (B) N-9 gel, (C) and sham-treatment, as well as blank (D) PEO, (E) PVA, and (F) PVP fibers for 24 hr. There was no indication of tissue inflammation or epithelial disruption from fiber administration, relative to untreated controls. Similar trends were observed for tissue specimens assessed $72 \mathrm{hr}$ post-administration (data not shown). 


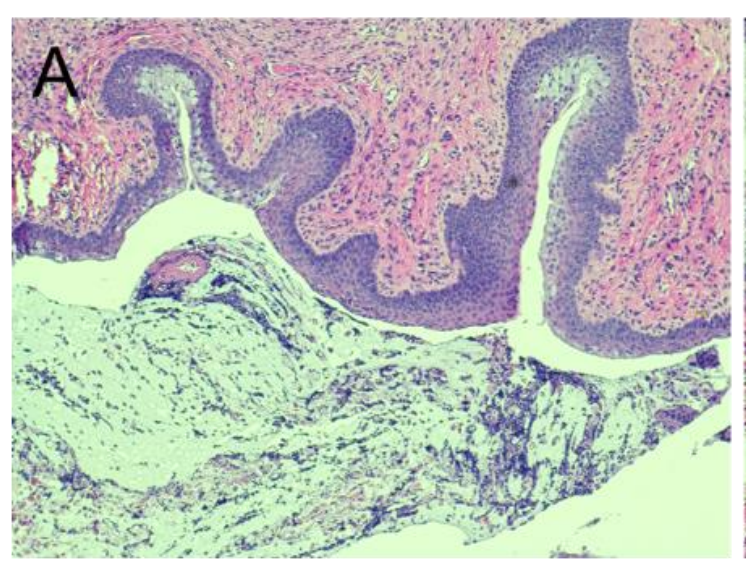

Blank PVA

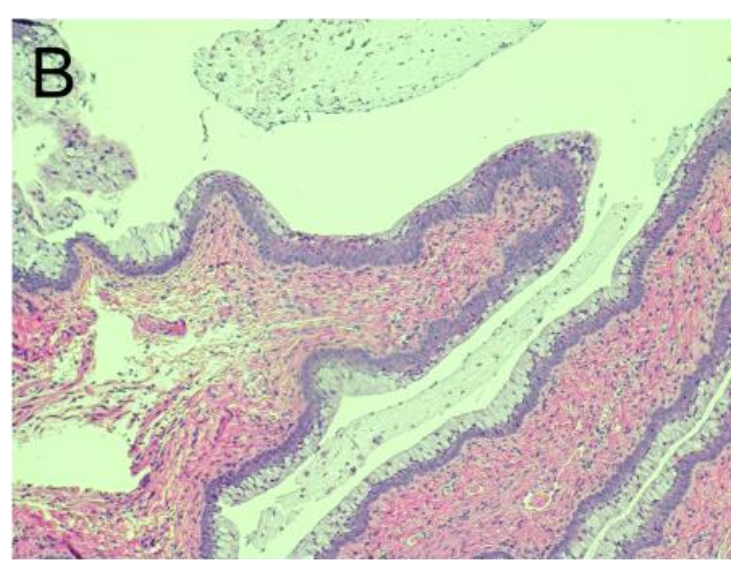

$4 \% \mathrm{~N}-9 \mathrm{Gel}$

Supplemental Figure 4.1. Images of H\&E stained tissue outliers. (A) A blank PVA fiber-treated tissue sample replicate demonstrated increased levels of mucin secretion and neutrophil presence; however, no disruption of the vaginal or cervical epithelium was observed. (B) In contrast, a replicate of $\mathrm{N}-9$ treated tissue exhibited inflammatory markers indicated by the presence of peripheral blood mononuclear cells, goblet cell fusions, and epithelial disruption. 
For N-9 treated mice, a slight increase in neutrophil accumulation was observed on the surface of the cervical squamous epithelium, relative to untreated controls, indicating minor topical damage (score range 1 to 2). Murine tissue from one $\mathrm{N}-9$ treated mouse exhibited increased inflammation, due to the presence of peripheral blood mononuclear cells, goblet cell fusions, and epithelial disruption, resulting in a score of 3 (Supplemental Figure 4.1B). As for sham-treated mice, there was a slight increase in neutrophil accumulation after $24 \mathrm{hr}$ in most samples, relative to untreated controls (score range 1 to 2 ), and one sham-treated replicate was noted for widespread neutrophil accumulation, indicating tissue repair (score 3). These alterations present in both N-9 and sham-treated controls were not observed in $72 \mathrm{hr}$ tissue samples, perhaps indicating transitory damage.

Cytokine expression was assessed from murine FRTs and vaginal lavages 24 and $72 \mathrm{hr}$ after blank fiber administration. Cytokine expression was compared to untreated, N-9 treated, and sham-treated mice based on previously published guidelines, in which a significant level of intravaginal inflammation results in a 2 to 5-fold increase in cytokine expression, relative to untreated controls ${ }^{69}$. Figure 4.8 summarizes the results from the cytokine analysis, indicating that blank fibers do not induce pro-inflammatory or immune-regulatory cytokine expression. In fibertreated tissue and vaginal lavage samples, cytokine levels were found to be comparable to tissue and washes collected from untreated control groups. Cytokine expression was similar in both 24 and $72 \mathrm{hr}$ samples, with 11 of 13 cytokines within a range of 2 to 5 -fold expression of untreated controls. A few exceptions were observed using the above criteria: vaginal lavages collected 24 
hr after PVA and PVP fiber administration demonstrated a 5-fold increase in IL-1a expression, while vaginal tissue collected $24 \mathrm{hr}$ after PEO administration demonstrated elevated levels of MIP1- $\alpha$ and PVP MIP-2, relative to untreated controls $(p<0.05)$. Additionally, vaginal tissue collected $72 \mathrm{hr}$ after PEO fiber administration demonstrated a 6 -fold increase in MCP-1 and MIP-1 $1 \alpha$ expression, relative to untreated controls $(p<0.05)$. For sham-treated controls, which simulated the administration method via tweezers only, increased cytokine expression was observed in $24 \mathrm{hr}$ tissue (MIP-2), $24 \mathrm{hr}$ wash (IL-1ß), and $72 \mathrm{hr}$ wash (IL-6, MCP-1), relative to untreated controls. N-9 treated samples demonstrated an increase in MIP-2 expression in $72 \mathrm{hr}$ wash samples only. Both IP-10 and INF- $\gamma$ were undetected in vaginal lavage or tissue samples. 


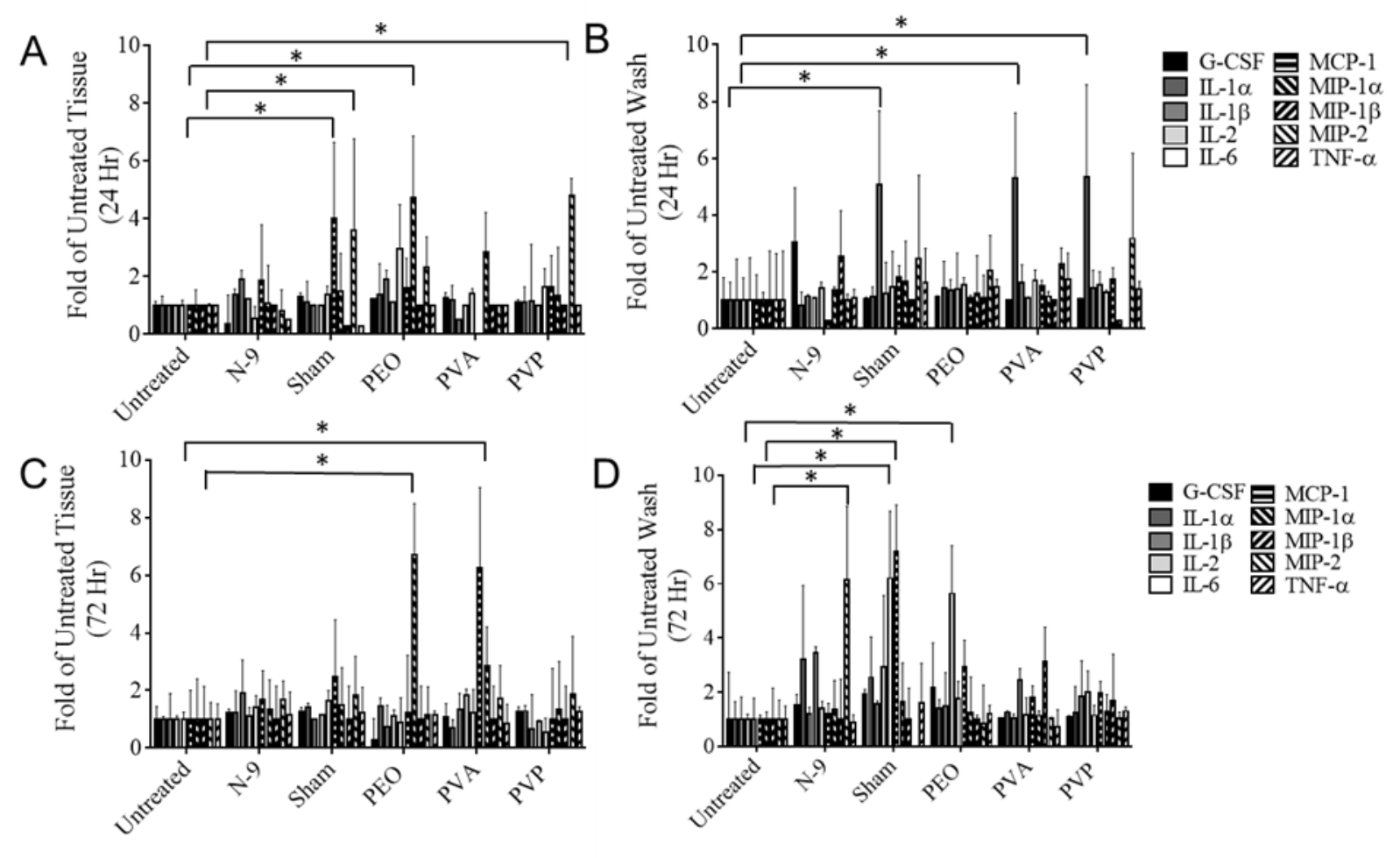

Figure 4.8. Cytokine expression from extracted murine tissue and vaginal lavages collected (A, B) 24 and (C, D) 72 hr after fiber administration. Cytokine expression was assessed concurrently with histology to determine the preliminary safety of rapid-release fibers in a murine model. Mice treated with blank fibers expressed similar cytokine levels relative to untreated controls, indicating that the presence of fibers does not induce inflammation or an inflammatory response $\left({ }^{*} p<0.05\right)$. 
An ELISA, used to confirm IL-1 $1 \beta$ levels, showed similar results, in that blank fibers induced negligible increases in cytokine expression relative to untreated controls $(p>0.05)$.

\section{Discussion}

In this study, electrospun fibers composed of hydrophilic polymers were evaluated as an alternative topical delivery platform to provide on-demand dualpurpose protection against HSV-2 and HIV-1 infections. GRFT PEO, PVA, and PVP fibers demonstrated complete in vitro efficacy against both HIV-1 and HSV-2 infections and exhibited comparable safety to free GRFT and untreated cells when tested in three vaginal cell lines. Furthermore, GRFT fibers were efficacious in an in vivo HSV-2 infection model, and demonstrated preliminary safety by maintaining macrostructural histology and similar cytokine expression, relative to untreated mice. Moreover, fibers preserve the activity of incorporated GRFT and substantiate the feasibility of electrospun fibers to provide an efficacious alternative platform for the intravaginal delivery of Griffithsin.

The antiviral lectin GRFT has been shown to potently inhibit a variety of viral infections, and has demonstrated particular promise in providing dual-purpose protection against HIV-1 and HSV-2 infections ${ }^{65,66}$. To date, GRFT has been primarily developed as a gel dosage form ${ }^{65}$; however, concerns surrounding gel administration, such as leakage and suboptimal user adherence in clinical trials, has prompted research into alternative delivery platforms. Other recent work has begun to evaluate the use of fast dissolving inserts (FDIs) comprised of the gelling 
agents carrageenan, hydroxyethyl cellulose, and xanthan gum ${ }^{73}$, for the delivery of active agents GRFT and carrageenan. These inserts have shown promise in providing on-demand release of agents, corresponding to immediate inhibition of SHIV infection in macaque models and HSV-2 and HPV inhibition in murine models. Another recent study examined the efficacy of poly(lactide-co-glycolide) GRFT nanoparticles (NPs) $)^{319}$ that co-encapsulate the ARV dapivirine, demonstrating synergistic protection against in vitro HIV-1 infections. While these studies show the potential of alternative GRFT delivery platforms, they may also be prone to challenges regarding leakage and ease of administration, prompting the development of solid dosage form alternatives that may be easily and discreetly administered to the FRT.

In this work, we envisioned a solid dosage form comprised of PEO, PVA, or PVP polymers, due to their established mucoadhesivity, biocompatibility, and hydrophilicity ${ }^{312,320}$. All three polymers have been used alone or in blends for drug delivery applications, particularly for the delivery of proteins and biologics ${ }^{321-327 . ~ I n ~}$ previous intravaginal delivery studies, mucoadhesive polymers were explored to increase active agent retention ${ }^{312,320}$. Specifically, one study investigated the use of PVA and PVP fibers to increase nanoparticle retention in the murine reproductive tract ${ }^{215}$, finding that nanoparticles incorporated within fibers demonstrated a 30 -fold increase in retention relative to free NPs ${ }^{215}$. In addition, their established biocompatibility, suggests their potential for translation, particularly in the reproductive microenvironment. Finally, the hydrophilic properties of PEO, PVA, and PVP enable fiber fabrication using aqueous 
solutions, which can help to retain GRFT ${ }^{328}$ and potentially other biologic activity. In future work we anticipate that the mucoadhesive properties of these polymers will increase GRFT retention within the FRT, thereby enhancing efficacy at potentially lower doses, relative to the administration of other dosage forms such as gels or nanoparticles. Future studies will be conducted to assess how mucoadhesion may enhance GRFT retention within the reproductive tract.

In these studies, the goal was to develop and preliminarily assess the safety and efficacy of rapid-release GRFT fibers in a murine model of HSV-2 infection. PEO, PVA, and PVP fibers all demonstrated high GRFT loading in both the 1 and $10 \% \mathrm{w} / \mathrm{w}$ GRFT formulations. These results are in agreement with other studies that have used hydrophilic fibers to incorporate proteins and other hydrophilic agents $^{329,330}$. The high encapsulation efficiency of these fibers is attributed in part to the favorable interactions between GRFT and polymers, specifically hydrogen bonding via hydrophilic functional groups ${ }^{312}$. This high loading, and moreover the preservation of GRFT activity, was further demonstrated in our in vitro efficacy studies where GRFT fibers completely inhibited both HIV-1 and HSV-2 infections in a dose-dependent manner, regardless of polymer formulation, and with similar $\mathrm{IC}_{50}$ values relative to free GRFT.

Previous studies have shown that GRFT exhibits picomolar potency against HIV-1, enabling $1 \% \mathrm{w} / \mathrm{w}$ GRFT fibers to completely prevent HIV-1 infection in vitro. However, the decreased number of oligomannose $\mathrm{N}$-linked glycans present on the surface of HSV-2, necessitates a higher dose of GRFT ${ }^{65}$ and correspondingly, GRFT fibers to prevent HSV-2 infection in vitro. Despite these differences in GRFT 
potency against HIV-1 and HSV-2, each fiber formulation provided complete, dualpurpose protection against in vitro infections.

Based on these successes, we sought to assess the efficacy and safety after fiber administration in a murine model of HSV-2 infection. In these studies, GRFT fibers were administered $4 \mathrm{hr}$ prior to HSV-2 infection. This administration time was based on surveys that studied female preference for topical delivery platforms, showing that an "ideal" platform should provide convenient and discreet administration, and can be applied hours prior to intercourse ${ }^{331}$. In line with previous studies testing GRFT gels ${ }^{65}$, all three GRFT fiber formulations provided comparable or enhanced protection in efficacy (LD90) studies (85-100\% survival), relative to free GRFT ( $85 \%$ survival). Moreover, the few mice that became infected showed decreased weight loss and overall slower progression of HSV-2 symptoms, relative to untreated/infected controls, indicating that GRFT fibers may reduce the severity of symptoms and alter the course of infection. This trend of reduced severity and delayed progression was also observed in previous in vivo GRFT studies further validating the efficacy profile of GRFT fibers ${ }^{332}$.

Interestingly, blank fiber administration protected up to $50 \%$ of total mice, relative to untreated/infected control mice, suggesting that physical fiber presence alone may provide a significant level of barrier-like prevention against infections. Previous studies by our group have demonstrated similar in vitro results, suggesting that the fiber itself may act as a barrier to viral infection ${ }^{75,87}$. Future work seeks to better define the fiber characteristics that contribute to this inhibition and utilize this information to improve fiber design. We hypothesize that fibers may 
be fabricated to serve as physical barriers to limit viral distribution within the FRT, in addition to providing release of incorporated active agents.

Last, we acknowledge that regardless of the protection imparted by GRFT fibers (or free GRFT), a small fraction of mice (averaging 1.3 and 3 mice of 20, for GRFT fiber and free GRFT-treated mice, respectively) became infected. Previous work has shown similar results, in which free GRFT significantly reduces the incidence of infection, but may not impart complete protection within a sample group ${ }^{65}$. Future work will assess dose-dependence and effects of different administration times of GRFT fibers on in vivo prevention.

In addition to efficacy, it is critical that fibers are safe to administer and minimize potential inflammatory responses ${ }^{333,334}$. All fiber formulations (PEO, PVA, and PVP) exhibited preliminary safety in in vitro and in vivo experiments. Histological analyses demonstrated the safety of these platforms in vivo, with the majority of fiber-treated tissue showing no signs of cervical or vaginal epithelium disruption, nor increased neutrophil accumulation relative to untreated controls. Additionally, cytokine expression in fiber-treated mice tissues and vaginal lavages demonstrated values within the normal range shown in previous studies, confirming the biocompatibility of both polymers and GRFT ${ }^{69,335}$. Although cytokine values from a few experimental and sham control group tissues were elevated relative to untreated controls (as seen with increased levels of IL-1a in PVA and PVP 24 hr vaginal lavages, and increased expression of MCP-1 and MIP-1 in PEO $72 \mathrm{hr}$ tissue samples), we attribute these incidences of a single elevated cytokine expression in samples to either vehicle administration or the inherent variability of 
in vivo studies. Previous studies have shown that inflammation is characterized by the overexpression of multiple cytokines. For example, increased IL-1 $\alpha$ expression, is typically associated with increased TNF- $\alpha$ or IL-1 $\beta$ expression ${ }^{186,336,337}$, therefore the singular overexpression of IL-1 $\alpha$ in both PVA and PVP $24 \mathrm{hr}$ lavage samples may not be a sufficient indicator of inflammatory response. Additionally, PEO $72 \mathrm{hr}$ tissue samples demonstrated increased expression of both MCP-1 and MIP-1, which causes increased localization of neutrophils ${ }^{338-340}$; however, no increased neutrophil accumulation was observed in histology samples during this time period.

In comparison to experimental groups, the sham-treated control group, which used sterile tweezers to mimic the method of fiber administration, showed similar cytokine profiles to blank fiber-treated tissue, with a slight increase in neutrophil accumulation in some histological sample replicates. These results suggest that this method of delivery may cause damage to the murine reproductive tract. While previous studies used positive displacement pipettes to intravaginally administer rolled-fibers, the amount of fiber administered with this method is limited $^{215}$. We believe the method of administration may be partially responsible for the observed increased cytokine expression, as well as the increased neutrophil accumulation seen in one of the blank PVA histology replicates (Supplemental Figure 4.1A). Future studies, will be conducted using alternative methods of fiber administration, to assess the impact of administration methods and to more closely represent more commonly used tampon-like administration packages. 
Concurrent with testing rapid-release GRFT fibers, N-9 gel was used as a positive control in our in vivo safety studies ${ }^{65}$. However, histology samples showed that a single administration of $\mathrm{N}-9$ only marginally increased neutrophil accumulation in tissue and no marked increase was observed in cytokine expression, relative to untreated or blank fiber-treated mice (Supplemental Figure 4.1B). Previous studies using $\mathrm{N}-9$ gels have shown that a single administration may result in transient damage within murine reproductive tissue, with the highest severity $4 \mathrm{hr}$ post-administration, followed by nearly complete recovery after $24 \mathrm{hr}^{341}$. This correlates with our in vivo efficacy results, in that mice give a single application of $\mathrm{N}-9$ gel show high HSV-2 infectivity, which may be due to the time of viral administration corresponding to the time at which $\mathrm{N}-9$ damage is most prominent.

In these studies, we have fabricated rapid-release GRFT fibers to provide on-demand protection against HIV-1 and HSV-2 infections, and have demonstrated the preliminary safety of GRFT fibers. Our goal is to create a delivery platform providing women an alternative viable solid dosage form that offers dualprotection against STI infections. Based on our cytokine work, future preclinical studies will explore alternative administration methods to ensure that platform administration does not enhance susceptibility to viral infection or cause off-target effects. Additionally, future studies will examine the window of protection provided by GRFT fibers by challenging with HSV-2 at different time points with respect to fiber administration and assessing the dose-dependent response to fiber administration. Moreover, we anticipate that GRFT fibers may enhance retention 
within the reproductive tract and in future work seek to study the retention and pharmacokinetics of GRFT delivered from these fibers after different durations, and how these concentration profiles relate to protection. Last, we anticipate that these rapid-release fibers may be used as a foundation to develop sustainedrelease multilayered fibers, which may provide extended release, decreased doses, and, potentially increased user adherence relative to existing delivery platforms. 


\section{CHAPTER 5}

\section{MULTIPURPOSE GRIFFITHSIN NANOPARTICLE-ELECTROSPUN FIBER COMPOSITES AGAINST HIV-1 AND HSV-2 INFECTIONS}

\section{Introduction}

A promising option to provide sustained topical delivery of GRFT may be polymeric electrospun fibers (EFs), which have been developed for FRT applications over the past decade ${ }^{342}$. Early studies showed that fibers comprised of cellulose acetate phthalate provided $\mathrm{pH}$-responsive release of tenofovir disoproxil fumarate to inhibit HIV-1 infection in vitro ${ }^{83}$. In other studies, polymeric fibers and fiber blends were fabricated to incorporate maraviroc, raltegravir, and tenofovir, and demonstrated sustained-release of these agents for up to 10 days $85,86,145,160,166$. While these fiber platforms have demonstrated sustaineddelivery of ARVs for many applications, to date, no fiber-based platforms have demonstrated the long-term delivery of biologics, such as GRFT, within the $\mathrm{FRT}^{38,107-109,204,211 .}$

In addition to electrospun fibers, polymeric nanoparticles (NPs) have been explored for a variety of FRT applications, and have been used to incorporate ARVs and other biologics (e.g., siRNA, antibodies) ${ }^{187,191,343-349}$. One of the key strengths of NPs for FRT delivery is that they can distribute throughout the 
$\mathrm{FRT}^{186,349,350 ;}$, however, NPs are often administered in gels or aqueous solutions, resulting in decreased agent retention due to mucus clearance. To address this challenge, we envisioned that a composite of nanoparticles and electrospun fibers (NP-EF) may provide an alternative delivery platform that integrates the attributes of each to prolong GRFT delivery. We hypothesized that multilayered fibers may act as a NP reservoir to increase NP retention and modulate GRFT release. Furthermore, in future work, we envision that this NP-EF composite platform may incorporate and modulate the delivery of multiple active agents to provide longterm, multi-mechanistic, and synergistic protection against multiple types of viral infections.

In previous work, our group developed $\mathrm{pH}$-responsive and surface-modified GRFT fibers that provided in vitro on-demand protection against HIV-1 infection $^{75,162}$. The goal of this study was to design and evaluate a novel NP-EF composite that incorporates GRFT to provide multipurpose and potentially longterm (> 1 month) protection against HSV-2 and HIV-1 infections. Here, NPs were electrospun into fibers, composed of a polyethylene oxide (PEO), polyvinyl alcohol (PVA), or polyvinylpyrrolidone (PVP) inner layer, surrounded by a polycaprolactone (PCL) outer layer (Figure 5.1). We hypothesized that NP-EF composites would provide sustained-release of GRFT and provide protection against a lethal HSV-2 challenge in a murine model. Furthermore, we hypothesized that the administration of NPs or NP-EFs would result in similar cytokine expression and tissue macrostructure, relative to untreated murine lavages and tissues. Importantly, the sustained-release of GRFT from NP-EF 
composites was demonstrated for up to 90 days, and completely inhibited HSV-2 and HIV-1 in vitro. Furthermore, GRFT NP-EF composites and GRFT NPs protected mice against a lethal HSV-2 challenge, suggesting that NP-EF composites may serve as an alternative platform for women that seek long-term dual-purpose prevention. 

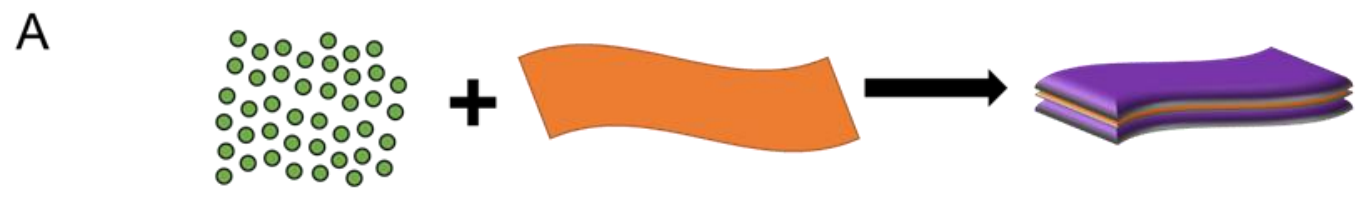

GRFT NPs Hydrophilic layer Multilayered NP-EF Construct

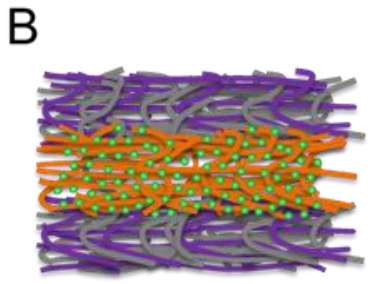

$1 \mathrm{~mL}$ Outer Layer

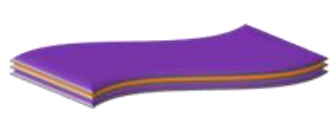

$1 \mathrm{~mL}$ Outer Layer

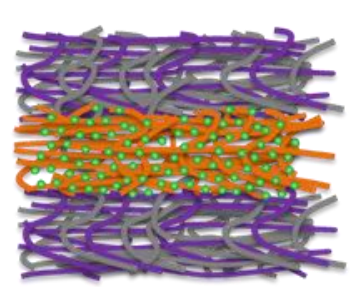

$3 \mathrm{~mL}$ Outer Layer

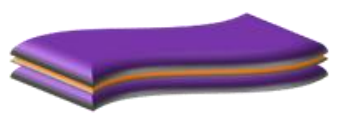

$3 \mathrm{~mL}$ Outer Layer

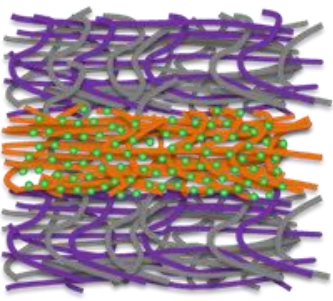

5 mL Outer Layer

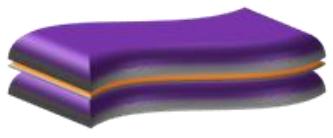

$5 \mathrm{~mL}$ Outer Layer

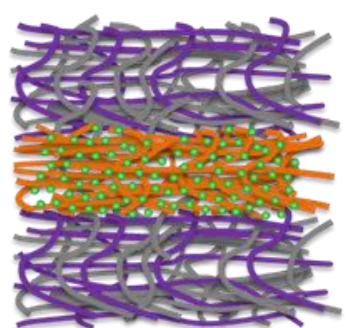

$7 \mathrm{~mL}$ Outer Layer

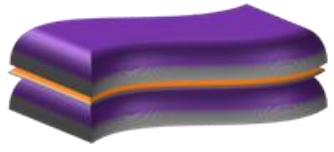

7 mL Outer Layer

Figure 5.1. (A) Schematic depicting the incorporation of NPs within hydrophilic fibers to create multilayered NP-EF composites. Multilayered fibers consist of an inner layer of hydrophilic polymer (here, PEO) that incorporates GRFT or C6 NPs, and outer hydrophobic layers of PCL to tune GRFT release from the incorporated NPs. (B) Schematic of NP-EF composites fabricated with varying outer layer thicknesses to modulate GRFT release. The multilayered fiber acts as a NP reservoir, conferring localized GRFT release from the composite. 


\section{Methods and Materials}

\section{Polymers and Solvents}

mPEG-PLGA (LG 50:50, 5,000 MW mPEG-50,000 MW PLGA) and PLGA (50:50, 0.55-0.75 dL/g, 31-57k MW) were purchased from PolySciTech (Lafayette, Indiana) and Lactel Absorbable Polymers (Cupertino, CA), respectively. Polymers including PCL (80,000 MW), PEO (600,000 MW), PVA (87-90\% hydrolyzed, 30,000-70,000 MW), and PVP (1,400,000 MW) were purchased from Sigma Aldrich (St. Louis, MO). Trifluoroethanol (TFE) and dichloromethane (DCM) were purchased from Thermo Fisher (Waltham, MA). Other chemicals, including dimethyl sulfoxide (DMSO) and thiazolyl blue tetrazolium bromide (MTT) were purchased from Sigma Aldrich. Griffithsin stock solution (12.0 mg/mL, in PBS) was kindly provided by Kentucky Bioprocessing (Owensboro, KY).

\section{Nanoparticle Synthesis}

mPEG-PLGA and PLGA nanoparticles containing GRFT were synthesized using the double emulsion solvent evaporation technique. First, $100 \mathrm{mg}$ PLGA or mPEG-PLGA were dissolved in $2 \mathrm{~mL}$ of DCM and incubated overnight. The next day, the polymer solutions were vortexed, and $200 \mu \mathrm{L}$ of GRFT stock solution (25, 50 , or $100 \mathrm{mg} / \mathrm{mL}$ in PBS) was added dropwise to synthesize NPs with a theoretical loading of 50,100 , or $200 \mu \mathrm{g} \mathrm{GRFT/mg} \mathrm{polymer.} \mathrm{GRFT-polymer} \mathrm{mixtures} \mathrm{were}$ sonicated and added to $2 \mathrm{~mL}$ of $5 \%$ PVA solution, sonicated again, and subsequently transferred to a beaker containing $50 \mathrm{~mL}$ of $0.3 \%$ PVA solution for 3 hr. The newly synthesized NPs were washed with Milli-Q water three times to 
remove residual PVA and solvent, followed by lyophilization and storage at $-20^{\circ} \mathrm{C}$ until use.

To assess NP loading within the NP-EF composite, Coumarin 6 (C6) NPs were synthesized as previously described ${ }^{351-355}$. Briefly, C6 was dissolved in DCM (15 $\mu \mathrm{g} \mathrm{C6/mg} \mathrm{polymer} \mathrm{or} 15 \mathrm{mg} \mathrm{C6}$ in $200 \mu \mathrm{L} \mathrm{DCM}$ ), followed by the dropwise addition of $200 \mu \mathrm{L}$ to the $2 \mathrm{~mL}$ polymer/DCM mixture. Afterward, the fabrication process of C6 NPs mirrored that of GRFT NPs, with sonication of the polymersolvent mixture, addition to $2 \mathrm{~mL}$ of $5 \%$ PVA solution, and subsequent transfer to $50 \mathrm{~mL}$ of $0.3 \%$ PVA solution for $3 \mathrm{hr}$.

\section{NP Loading in Hydrophilic (Inner Layer) Fibers}

Hydrophilic fibers, incorporating GRFT mPEG-PLGA NPs, were fabricated by first adding PEO ( $5 \% \mathrm{w} / \mathrm{w})$, PVA $(20 \% \mathrm{w} / \mathrm{w})$, and PVP $(20 \% \mathrm{w} / \mathrm{w})$ to scintillation vials containing $1 \mathrm{~mL}$ Milli-Q water and were incubated overnight. Blank fiber controls were electrospun with a mandrel-to-syringe distance of $20 \mathrm{~cm}$, flow rate of $0.25-0.60 \mathrm{~mL} / \mathrm{hr}$, and a voltage of $15-20 \mathrm{kV}$. To determine the loading capacity of NPs within the fibers, C6 NPs were electrospun in fibers at 1,2,10, or $20 \% \mathrm{w} / \mathrm{w}$ (NP/EF weight ratio). For GRFT NP-EF composites, PEO fibers were electrospun with 1 or $20 \%$ w/w GRFT NPs. Electrospinning parameters were adjusted for GRFT and C6 NP-EFs by changing the flow rate to $0.2 \mathrm{~mL} / \mathrm{hr}$ and voltage to 20 $25 \mathrm{kV}$.

\section{NP-EF Composite Synthesis}


Polycaprolactone $(11 \% \mathrm{w} / \mathrm{w}$ or $152 \mathrm{mg} / \mathrm{mL})$ was dissolved in $7 \mathrm{~mL}$ TFE and incubated overnight. Different PCL solutions were electrospun using volumes of 1 , 3, 5 or $7 \mathrm{~mL}$ to create composites with varying outer layer thicknesses. Similar electrospinning conditions were used with the mandrel-to-syringe distance set to $15 \mathrm{~cm}$, flow rate of $2.2 \mathrm{~mL} / \mathrm{hr}$, and voltage of $20 \mathrm{kV}$. Pre-massed NP-PEO fiber sheets were placed on the freshly electrospun PCL layer and an additional PCL layer was electrospun on top to create a PCL-(NP/PEO)-PCL multilayered structure (Figure 5.1), and fibers were desiccated overnight prior to characterization.

\section{NP and NP-EF Composite Morphology}

Scanning electron microscopy (SEM) was used to characterize the physical structure and morphology of NPs, EFs, and NP-EF composites. Individual hydrophilic and hydrophobic fiber layers as well as multilayered composites were cut into $5 \mathrm{~mm}$ pieces and placed on double-sided adhesive carbon tabs (Ted Pella Inc., Redding, CA, USA), which were then adhered to aluminum stubs. Nanoparticles were similarly adhered to carbon tabs and all samples were sputter coated with a thin gold alloy using a Bio-Rad E5100 sputter coat system (Hercules, CA). Samples of composite cross sections were prepared by slicing composites

into 2 to $5 \mathrm{~mm}$ sections and adhering sections sideways to the aluminum stubs. Sample images were acquired in triplicate using a Supra 35 SEM (Zeiss, Oberkochen, Germany). Fiber and composite images were captured using an accelerating voltage of $8 \mathrm{kV}$, with magnifications of 5,000 - to $10,000 \mathrm{x}$, while NP images were captured with a voltage of $10 \mathrm{kV}$, and magnification of 20,000 to 
23,000x. The mean diameters of NPs and fibers were determined using ImageJ software (NIH, Bethesda, Maryland), by measuring 50 line elements per SEM image.

\section{Quantification of GRFT NP, GRFT NP-EF, and C6 NP-EF Loading}

The loading of GRFT in NPs and NP-EFs was assessed using an enzymelinked immunosorbent assay (ELISA). Griffithsin loading and encapsulation efficiency (defined as [actual loading $\div$ theoretical loading] $\times 100 \%$ ) was determined for NPs and NP-EF composites. To determine loading, GRFT NPs and NP-EF composites (2 to $4 \mathrm{mg}$ ) were dissolved in $1 \mathrm{~mL} \mathrm{DCM}$, and vortexed for 30 s. Griffithsin was extracted from the DCM into $500 \mu \mathrm{L}$ Tris-EDTA (TE) buffer, vortexed, and centrifuged at $18,500 \times \mathrm{g}$ for $5 \mathrm{~min}$. TE buffer was collected, and the process was repeated to collect GRFT into a final volume of $1 \mathrm{~mL}$. All extracts were analyzed using ELISA as previously described ${ }^{162}$.

For the ELISA, 96-well Nunc Maxisorp plates were incubated overnight with $100 \mu \mathrm{L}$ coating buffer (250 ng/mL gp120 in PBS). Coating buffer was removed from the plates, and $300 \mu \mathrm{L}$ blocking buffer $(0.05 \%$ Tween-20 and $3 \% \mathrm{w} / \mathrm{v}$ bovine serum albumin in PBS) was added to the wells for $2 \mathrm{hr}$. After incubation, the plates were washed with PBST (0.05\% Tween-20 in PBS) using a Gardner Denver Multiwash III plate washer (Milwaukee, WI), and free GRFT standards (100 $\mu \mathrm{L}, 0.2$ to $120 \mathrm{ng} / \mathrm{mL}$ ) or sample extracts were added to each well. Plates were incubated for $1 \mathrm{hr}$ at $37^{\circ} \mathrm{C}$, followed again with a PBST wash. Dilutions of goat anti-GRFT primary antibody (1:10,000, kindly provided by Dr. Kenneth Palmer, University of 
Louisville) and rabbit anti-goat IgG-HRP secondary antibody (1:20,000, Sigma Aldrich) were subsequently added to each well in $100 \mu \mathrm{L}$ volumes and incubated for $1 \mathrm{hr}$ at $37^{\circ} \mathrm{C}$ to detect bound GRFT. After incubation, both antibody solutions were rinsed and $100 \mu \mathrm{L}$ of $\mathrm{KPL}$ SureBlue TMB microwell peroxidase substrate (Sera Care, Milford, MA, USA) was added to each well for $90 \mathrm{~s}$, followed by quenching with $100 \mu \mathrm{L}$ of $1 \mathrm{~N} \mathrm{H}_{2} \mathrm{SO}_{4}$ (Thermo Fisher). Plate absorbance was measured at $450 \mathrm{~nm}$ on a Synergy HT reader (BioTek, Winooski, VT, USA) and data were analyzed using Prism (GraphPad Software, La Jolla, CA).

The loading of C6 NPs within NP-EFs was assessed via fluorescence. First, C6 NP-EFs (2 to $4 \mathrm{mg}$ ) were dissolved in $1 \mathrm{~mL} \mathrm{DMSO}$, and vortexed for $1 \mathrm{~min}$ to ensure complete composite dissolution. Composite dilutions were added (100 $\mu \mathrm{L})$ to 96-well plates. C6 NPs (3mg) were similarly dissolved in DMSO, and NP dilutions were used to generate a standard curve (ranging $6 \mathrm{ng} / \mathrm{mL}$ to $100 \mu \mathrm{g} / \mathrm{mL}$ ), while dissolved blank NPs were used as background controls. Plates were read at excitation and emission wavelengths of 443 and $494 \mathrm{~nm}$ using a Synergy HT reader.

\section{Quantification of GRFT Release from NPs and NP-EF Composites}

The sustained-release of GRFT from NPs and NP-EF composites was assessed for 30 and $90 \mathrm{~d}$, respectively. GRFT PLGA and mPEG-PLGA NPs (3 to $5 \mathrm{mg}$ ) were aliquoted to $1.5 \mathrm{~mL}$ centrifuge tubes that contained $1 \mathrm{~mL}$ of simulated vaginal fluid $(S V F)^{246}$, while NP-EF composites were placed in $10 \mathrm{~mL}$ scintillation vials and incubated in $3 \mathrm{~mL}$ SVF to ensure complete submersion. All samples were 
placed in a shaker at $37^{\circ} \mathrm{C}$ and $150 \mathrm{rpm}$, and at specific time points, the SVF was collected and replaced with fresh SVF. To collect NP release eluate, samples were centrifuged at $18,500 \times \mathrm{g}$ at $4^{\circ} \mathrm{C}$ for 10 min prior to supernatant collection.

\section{Cell Lines and Virus}

TZM-bl cells, obtained from the National Institutes of Health AIDS Research and Reference Reagent Program (ARRRP), are engineered HeLa cells that express CD4, CCR5 and CXCR4 receptors, and were used to assess in vitro HIV1 infectivity. TZM-bl cells are highly permissive to infection by most HIV-1 and pseudovirus strains, and contain HIV-activated Tat-driven luciferase, allowing for precise quantification of infection. TZM-bl cells were cultured in Dulbecco's Modified Eagle medium (DMEM) containing 10\% fetal bovine serum (FBS), $25 \mathrm{mM}$ HEPES, and $50 \mu \mathrm{g} / \mathrm{mL}$ gentamicin (Thermo Fisher).

The Env-pseudotype HIV-1 was produced in house by introducing HEK293T/17 cells (ATCC, Rockville MD) with both an envelope (env)-expressing plasmid (CCR5-tropic clade A strain, Q769.h5) and an env-deficient HIV-1 backbone vector (pNL4.3AEnv-Luc). Both plasmids were obtained from the $\mathrm{NIH}$ AIDS Reagent Program (catalog\# 11884 and 3418). Cells were maintained in minimum essential medium (MEM) supplemented with 10\% FBS, $1 \%$ penicillin (100 $\mu \mathrm{g} / \mathrm{mL})$, and $1 \%$ streptomycin $(100 \mu \mathrm{g} / \mathrm{mL})$ (VWR Radnor, PA).

To assess HSV-2 infection in plaque assays, Vero E6 cells (African green monkey kidney) and HSV-2 (4674) were kindly provided by Dr. Kenneth Palmer 
from the University of Louisville. Cells were maintained in MEM supplemented with $10 \%$ FBS, and $1 \%$ penicillin and streptomycin.

Finally, vaginal keratinocyte (VK2/E6E7), ectocervical (Ect1/E6E7), and endocervical (End1/E6E7) cell lines were used to assess fiber cytotoxicity (all cells courtesy of Dr. Kenneth Palmer, originally from ATCC, Rockville MD). VK2/E6E7 (VK2), Ect1/E6E7 (Ect1), and End1/E6E7 (End1) are well-characterized immortalized cell lines derived from normal human vaginal, ectocervical, and endocervical epithelia, respectively, and are representative of the cell types found within the FRT. Cells were maintained in keratinocyte serum-free medium (KSFM) supplemented with recombinant human epidermal growth factor $(0.1 \mathrm{ng} / \mathrm{mL})$, bovine pituitary extract $(50 \mu \mathrm{g} / \mathrm{mL})$, calcium chloride $(0.4 \mathrm{mM})$ (Thermo Fisher), with $1 \%$ penicillin and streptomycin. During cell trypsinization, plating, and cell counting, cells were neutralized with 1:1 DMEM:Nutrient Mixture F-12 media (Thermo Fisher) with $10 \%$ FBS, and $1 \%$ penicillin/streptomycin.

\section{In Vitro HIV-1 Inhibition}

The activity of GRFT NPs and NP-EF composites against HIV-1 infection was assessed using an in vitro HIV-1 inhibition assay as previously described $^{75,87,161,162}$. HIV-1 inhibition was determined as a function of reduction in luciferase reporter gene expression after a single round of infection with pseudotyped HIV-1 in TZM-bl cells. The optimal virus dilution (1:16) was established to yield $\geq 96,800$ relative luminescence units (RLUs). Briefly, GRFT NP or NP-EF eluates were prepared in either PBS or DMEM $(1.0 \mathrm{mg} / \mathrm{mL})$, followed 
by 1:2 serial dilutions with DMEM to a final volume of $50 \mu \mathrm{L}$ within a 96 -well plate. One hundred microliters of TZM-bl cells (10,000 cells in DMEM supplemented with $10 \mu \mathrm{g} / \mathrm{mL}$ DEAE-dextran) was added to each well, followed by the addition $50 \mu \mathrm{L}$ of HIV-1 pseudovirus dilution. Infected cells administered NPs or NP-EFs were incubated at $37^{\circ} \mathrm{C}$ for $48 \mathrm{hr}$ and compared to the inhibition obtained with free GRFT-treated/infected (stock concentration $50 \mu \mathrm{g} / \mathrm{mL}$ ), untreated/infected, and untreated/uninfected control groups.

After $48 \mathrm{hr}, 100 \mu \mathrm{L}$ of culture medium was removed from each well. Luminescence was measured using the Bright-Glo luciferase assay (Promega Corporation, Madison, $\mathrm{WI}$ ) by adding $100 \mu \mathrm{L}$ Bright-Glo reagent solution to each well for $5 \mathrm{~min}$ and recording relative luminescence units (RLUs, Synergy HT reader, BioTek). All RLU values were corrected by subtracting the RLU of untreated/uninfected cells from the RLUs of treated/infected cells. The percent virus inhibition was determined by normalizing the corrected RLUs of treated/infected cells to corrected untreated/infected cells with the following formula: \% Infection $=[$ (sample RLU - untreated uninfected cells $) \div($ untreated infected cells - untreated uninfected cells)] $\times 100 \%$. The antiviral activity is reported as the half maximal inhibitory concentration $\left(\mathrm{IC}_{50}\right)$ and was calculated by comparing the sample luminescence intensity to the luminescence intensity from untreated/infected controls.

\section{In Vitro HSV-2 Inhibition}


HSV-2 plaque assays were conducted to determine the in vitro efficacy of GRFT mPEG-PLGA NPs against HSV-2 infection. Briefly, Vero E6 cells were seeded at a density of 600,000 cells/well in a 6 -well flat bottom plate and grown for $24 \mathrm{hr}$ to complete confluence. Prior to cell infection, 20 mg of GRFT NPs were added to $20 \mathrm{~mL}$ complete plating media (1\% FBS MEM). Once the cells were fully confluent, the growth media was removed and replaced with $2 \mathrm{~mL}$ dilutions per well of GRFT NPs, followed by the introduction of HSV-2 (10 $\mu \mathrm{L}$ containing 3,000 PFU per well) after $1 \mathrm{hr}$ of treatment. Free GRFT $(2,000 \mu \mathrm{g} / \mathrm{mL})$ corresponding to the concentration needed to provide complete inhibition and untreated/infected cells, were used as negative and positive controls of HSV-2 infection. Untreated/uninfected cells were used as additional negative controls.

After HSV-2 infection, plates were incubated for $48 \mathrm{hr}$ at $37^{\circ} \mathrm{C}$. After incubation, media was removed, cells were fixed for $10 \mathrm{~min}$ with $1.5 \mathrm{~mL}$ of methanol, stained with crystal violet $(0.1 \%$ crystal violet in 80:20 water: ethanol solution), and incubated for 30 min. Finally, plates were washed with Milli-Q water, dried, and plaques were counted. Plaque numbers from experimental groups were normalized to the number of plaques in untreated/infected cells ( 280-300 plaques). Samples were analyzed in triplicate, and GraphPad Prism software was used to determine and compare the half maximal inhibitory concentration $\mathrm{IC}_{50}$ values of GRFT NPs to free GRFT.

\section{In Vitro Cytotoxicity}


Vaginal epithelial (VK2/E6E7), ectocervical (Ect1/E6E7), and endocervical (End1/E6E7) cells were incubated with either blank NPs, blank NP-EFs, GRFT NPs, or GRFT NP-EF composites to assess the in vitro cytotoxicity of GRFT delivery platforms. Cells were plated at a density of 50,000 cells/well in 96-well plates and incubated in triplicate with either 0.5 or $1 \mathrm{mg} / \mathrm{mL} \mathrm{NPs}$ in media. Composites were tested by incubating cells with $0.5 \mathrm{mg} \mathrm{NP}$-EF pieces $(2.5 \mathrm{mg} / \mathrm{mL}$ in media). No treatment (media alone) and 10\% DMSO were used as positive and negative controls of cell viability, respectively. After 24,48 , and $72 \mathrm{hr}$ incubation, $20 \mu \mathrm{L}$ of MTT reagent was added to the cells and incubated for an additional $4 \mathrm{hr}$. Cells were lysed overnight with $100 \mu \mathrm{L}$ lysis buffer (10\% sodium dodecyl sulfate in $0.01 \mathrm{M}$ hydrochloric acid). Absorbance values were measured at $570 \mathrm{~nm}$ the following day and normalized to untreated cell absorbance to obtain percent viability.

\section{In Vivo HSV-2 Efficacy}

All in vivo experiments were approved by the University of Louisville's Institutional Animal Care and Use Committee (IACUC-17135) prior to testing. Animal studies were conducted using 5-week-old female BALB/c mice (Jackson Laboratory, Bar Harbor, ME) to evaluate the efficacy of mice to HSV-2 infection after administration of GRFT NPs and NP-EF composites $(n=20)$. For these studies, mice were subcutaneously injected with $3 \mathrm{mg}$ Depo-Provera (Revive, Madison, NJ) 5 days prior to vehicle administration to induce the diestrous stage of their cycle. All materials administered to mice were UV-sterilized for $15 \mathrm{~s}$ prior to use. Mice were administered $0.5 \mathrm{mg}$ blank or GRFT NPs diluted in sterile PBS 
(20 $\mu \mathrm{L}$ of $25 \mathrm{mg} / \mathrm{mL}$ ) or $20 \% \mathrm{w} / \mathrm{w}$ GRFT NP-EF composites ( $5 \mathrm{mg}$ ) under isoflurane anesthesia. Untreated/uninfected mice and mice treated with $20 \mu \mathrm{g}$ free GRFT (20 $\mu \mathrm{L}$ of $1,000 \mu \mathrm{g} / \mathrm{mL}$ ) served as negative controls of infection, while untreated/infected mice served as a positive control for infection. Blank mPEGPLGA nanoparticles were administered as an additional control group in efficacy studies. A single challenge of HSV-2 LD90 (here, 5,000 PFU, HSV-2(4674) was given $24 \mathrm{hr}$ after vehicle administration, followed by 14 day observation using an established 4-point scale to monitor infection progression ${ }^{69,252,318}$. Each day, mice were weighed and examined for neurological and epithelial damage. The study concluded $14 \mathrm{~d}$ after HSV-2 challenge, mice were euthanized, and survival was assessed. Kaplan-Meier survival curves were generated from the data, followed by log-ranked post hoc tests to assess the statistical significance between groups.

\section{In Vivo Safety}

Additional studies were conducted to evaluate the safety of blank NPs and NP-EF composites in vivo. NPs and NP-EFs were UV-sterilized for $15 \mathrm{~s}$, and intravaginally administered to mice under isoflurane anesthesia, at the same doses ( 0.5 and $5 \mathrm{mg}$, respectively) that were evaluated in the efficacy study. Control groups included uninfected mice that were untreated, treated with $20 \mu \mathrm{L}$ free GRFT in PBS $(1,000 \mu \mathrm{g} / \mathrm{mL})$, or treated with $40 \mu \mathrm{L}$ of N-9 gel. An additional "sham" control was used to evaluate the effects of tweezer administration alone. Mice were euthanized after 24 or $72 \mathrm{hr}$ of vehicle administration ( $\mathrm{n}=3$ per time point) and reproductive tracts and vaginal lavages were collected and stored at $-80^{\circ} \mathrm{C}$ following mouse euthanasia. 
The structural integrity of the murine reproductive tracts was evaluated with histology. Collected tissue samples were washed with PBS, and subsequently fixed with $4 \%$ paraformaldehyde. The uterine horns, uterine body, and vagina were resected, fixed in $10 \%$ formalin, embedded in paraffin and sliced and stained with hematoxylin and eosin (H\&E) for histological examination. Similarly, tissue samples and vaginal lavages were collected and analyzed for cytokine expression. The histology of sample cross-sections was assessed by a pathologist blinded to treatment groups.

Cytokine expression from murine reproductive tracts and vaginal lavages was determined using Luminex assays. First, $20 \mu \mathrm{L}$ of T-Per solution (Thermo Fisher) containing $1 \%$ Halt Protease Inhibitor Cocktail (Thermo Fisher) was added per $1 \mathrm{mg}$ of reproductive tissue. Approximately 20 zirconia/silica beads (BioSpec Productions) were added to each sample, followed by homogenization at $4,500 x$ for 3 min (Bertin, France). Homogenized samples were cooled on ice for $5 \mathrm{~min}$, and centrifuged at $10,000 \times \mathrm{g}$ at $4^{\circ} \mathrm{C}$ for $5 \mathrm{~min}$. Sample supernatants were collected, aliquoted, and stored at $-80^{\circ} \mathrm{C}$ for further study. Prior to Luminex analysis, interleukin 1 beta (IL-1 $\beta$ ) levels were tested in the reproductive specimens using specific ELISA Ready-SET-Go! kits (Thermo Fisher) to confirm cytokine concentration ranges prior to the Luminex assays. Luminex assays were subsequently used to quantify cytokine levels, including G-CSF, INF-y, IL-1a, IL$1 \beta$, IL-2, IL-6, IP-10, MCP-1, MIP-1 $\alpha$, MIP1- $\beta$, MIP-2, and TNF- $\alpha$ from murine tissues and lavages. These cytokines were selected for assessment based on intravaginal inflammatory markers quantified in previous studies ${ }^{65,69}$. 


\section{Statistical Analysis}

Unless otherwise noted, all in vitro experiments were conducted in triplicate, with 3 replicates per sample. Statistical analysis of samples for delivery vehicle morphology, loading, in vitro assays, and in vivo safety studies was performed by using one-way ANOVA with the Bonferroni post hoc test $(p<0.05)$. For murine efficacy studies, log-ranked post hoc tests were conducted to determine statistical significance as a function of treatment group and survival outcome.

\section{Results}

\section{Nanoparticle Characterization}

PLGA and mPEG-PLGA NPs were synthesized with 50, 100, or $200 \mu \mathrm{g}$ GRFT per mg polymer. NPs were assessed for morphology, total GRFT loading, and sustained-release. The morphology and diameter of GRFT NPs, relative to blank NPs, were evaluated using SEM (Figure 5.2) and ImageJ software (Supplemental Figure 5.1). All NP formulations possessed spherical morphologies, with diameters ranging from 72 to $131 \mathrm{~nm}$. Blank nanoparticles demonstrated the largest diameters, with average PLGA and mPEG-PLGA NP diameters of 118 and $128 \mathrm{~nm}$ respectively. The addition of $50 \mu \mathrm{g} / \mathrm{mg}$ GRFT to PLGA and mPEG-PLGA NPs decreased NP diameters to 97 and $102 \mathrm{~nm}$, respectively, while the addition of $100 \mu \mathrm{g} / \mathrm{mg}$ GRFT resulted in average diameters of 131 and $78 \mathrm{~nm}$. The smallest PLGA and mPEG-PLGA NP diameters (72 and $74 \mathrm{~nm}$ ), were achieved with the addition of $200 \mu \mathrm{g} / \mathrm{mg}$ GRFT. Overall the incorporation of GRFT corresponded to a decrease in NP diameter. 


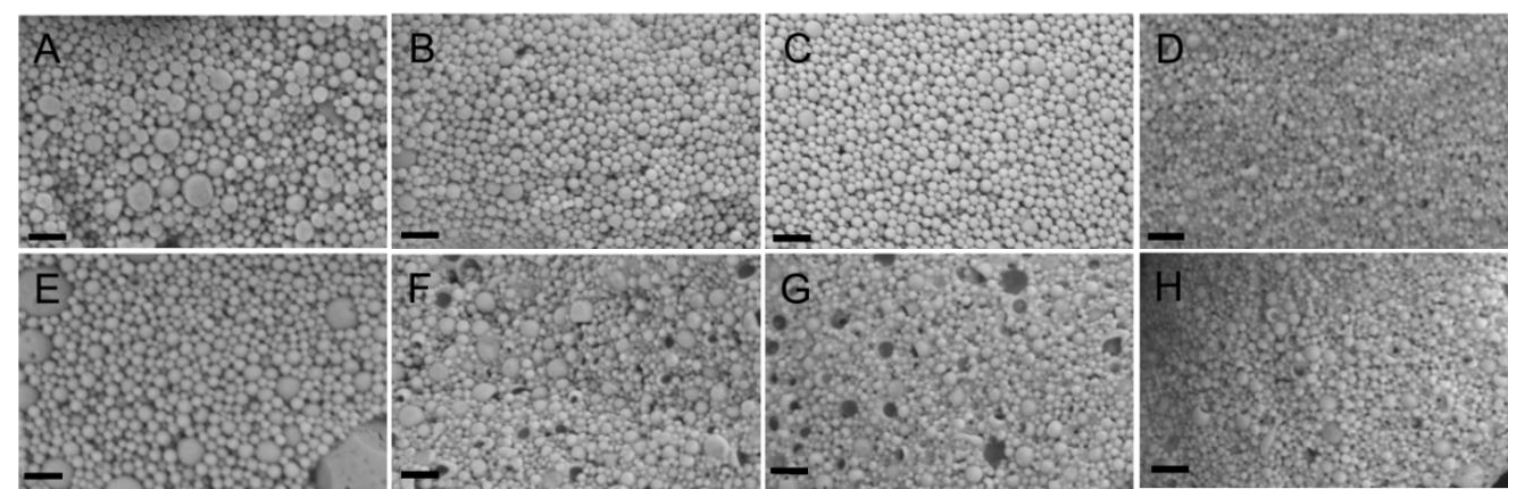

Figure 5.2 Scanning electron microscopy images of (A) blank, (B) 50, (C) 100, and (D) $200 \mu \mathrm{g} / \mathrm{mg}$ GRFT PLGA NPs, and (E) blank, (F) 50, (G) 100, and (H) 200 $\mu \mathrm{g} / \mathrm{mg}$ GRFT mPEG-PLGA NPs. Scale bars represent $100 \mathrm{~nm}$. 


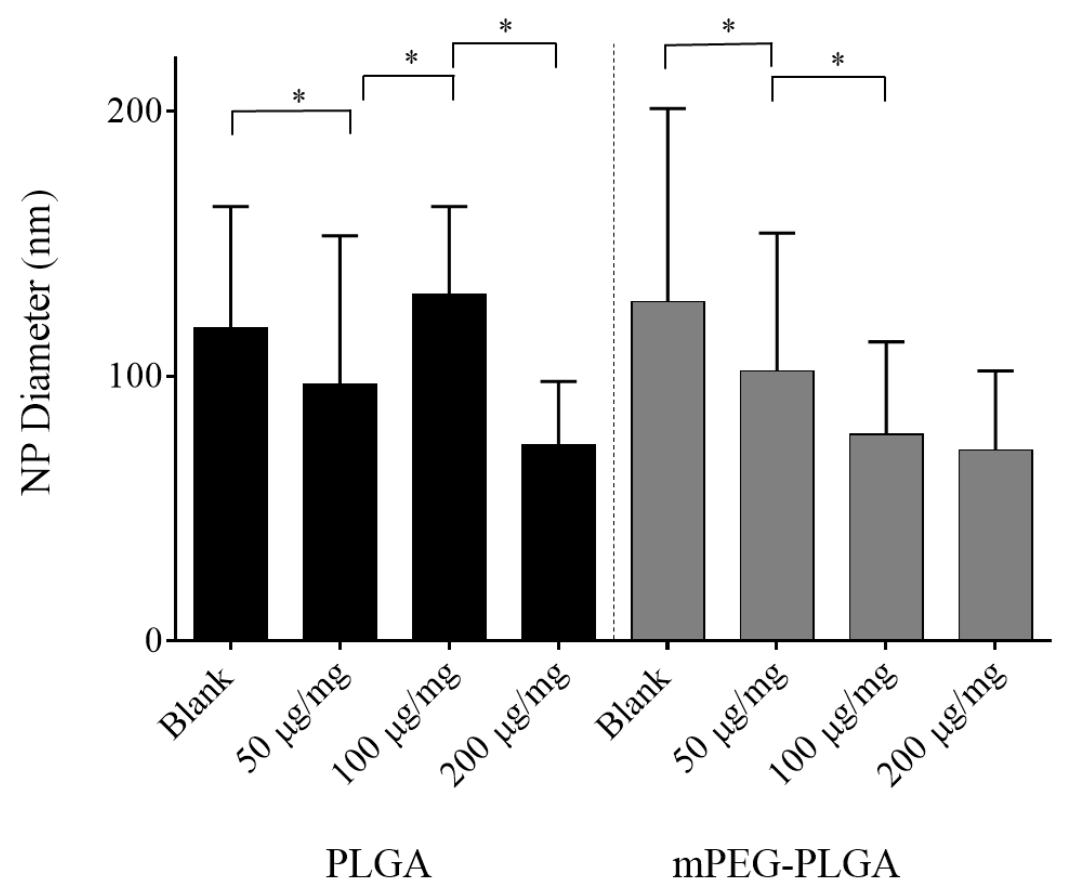

Supplemental Figure 5.1. Characterization of the average diameter of blank and GRFT NPs. NP diameters were determined using SEM and ImageJ software. Statistical significance between groups represented by * $(p<0.05)$. 
The loading of GRFT in PLGA and mPEG-PLGA NPs is shown in Table 5.1. The addition of 50,100 , and $200 \mu \mathrm{g}$ GRFT/mg PLGA NPs resulted in $23.6 \pm$ $1.4,42.4 \pm 3.1$, and $77.8 \pm 4.6 \mu \mathrm{g}$ GRFT/mg PLGA respectively, while mPEGPLGA NPs demonstrated consistently higher total GRFT loading of $49.6 \pm 1.9$, $63.6 \pm 15.1$, and $171.1 \pm 21.5$, for each formulation, respectively. 
Table 5.1. Griffithsin loading in mPEG-PLGA and PLGA NPs. Griffithsin extracts from NPs were assessed using ELISA. Increased theoretical loading resulted in increased actual loading. Three NP batches were fabricated for each formulation, with GRFT loading expressed as mean \pm standard deviation of triplicate readings of each NP batch. Statistical significance was assessed using one-way ANOVA with the Bonferroni post hoc test.

\begin{tabular}{|c|c|c|c|}
\hline \multirow{2}{*}{ Polymer } & $\begin{array}{c}\text { Theoretical Loading } \\
\text { GRFT/NP }(\boldsymbol{\mu g} / \mathbf{m g})\end{array}$ & $\begin{array}{c}\text { Actual Loading } \\
\text { GRFT/NP }(\boldsymbol{\mu g} / \mathbf{m g})\end{array}$ & $\begin{array}{c}\text { Encaps ulation Efficiency } \\
\mathbf{( \% )}\end{array}$ \\
\hline \multirow{3}{*}{ PLGA NP } & 50 & $23.6 \pm 1.4$ & $47.2 \pm 2.9$ \\
\cline { 2 - 4 } & 100 & $42.4 \pm 3.1$ & $42.4 \pm 3.1$ \\
\cline { 2 - 4 } & 200 & $77.8 \pm 4.6$ & $38.9 \pm 2.3$ \\
\hline \multirow{3}{*}{ mPEG-PLGA NP } & 50 & $46.6 \pm 1.9$ & $93.2 \pm 1.7$ \\
\cline { 2 - 4 } & 100 & $63.6 \pm 15.1$ & $63.6 \pm 15.1$ \\
\cline { 2 - 4 } & 200 & $171 \pm 21.5$ & $85.6 \pm 11.0$ \\
\hline
\end{tabular}


During NP release studies, an initial burst release was observed from both PLGA and mPEG-PLGA NPs through one week, followed by minimal GRFT release from 1 to 4 wk (Figure 5.3). Increased release was seen from mPEGPLGA NPs, relative to PLGA NPs, with 66.5 and $38.6 \mu \mathrm{g} / \mathrm{mg}$ GRFT released from $200 \mu \mathrm{g} / \mathrm{mg} \mathrm{mPEG}-\mathrm{PLGA}$ and PLGA NPs within 4 wk. Finally, as expected, the amount of GRFT release corresponded to the amount of GRFT loading for both polymers, with $200 \mu \mathrm{g} / \mathrm{mg} \mathrm{mPEG}-\mathrm{PLGA}$ NPs releasing the most GRFT over 4 wk. 

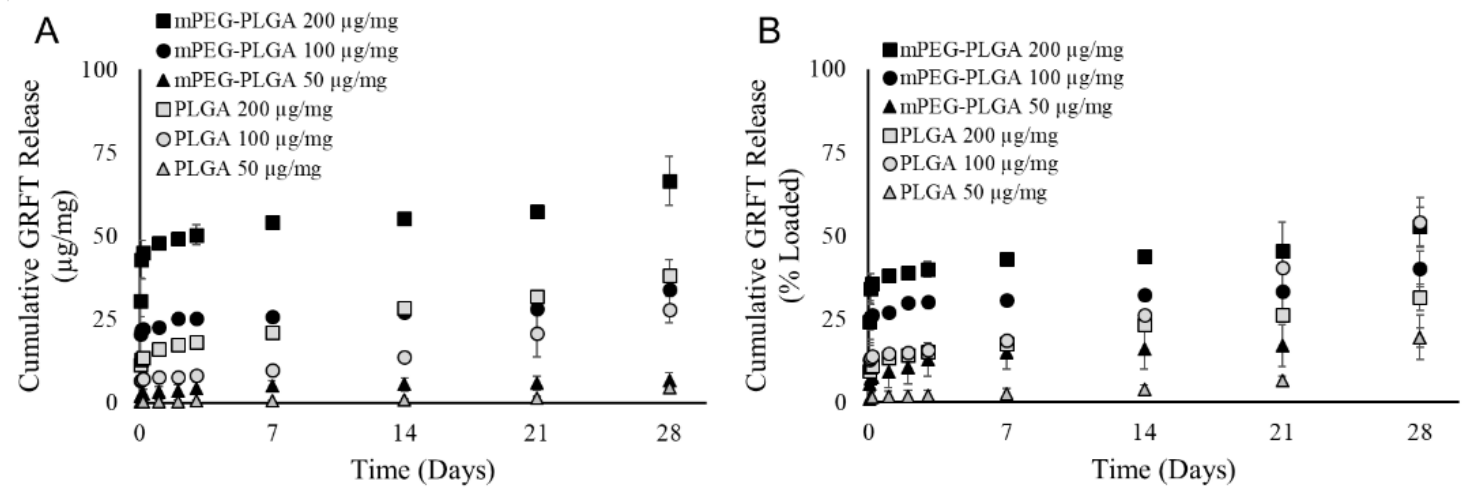

Figure 5.3. The cumulative release of GRFT from mPEG-PLGA and PLGA NPs shown as (A) total GRFT release or $(B)$ the percent of total loading. Increased amounts of GRFT were released from mPEG-PLGA NPs relative to PLGA NPs, and total release corresponded with GRFT loading for each formulation. Release values are shown as the mean \pm standard deviation of three independent NP batches. 


\section{Nanoparticle-Electrospun Fiber Composite Characterization}

Studies were conducted to assess the maximum loading that could be attained by adding C6 NPs to PEO, PVA, and PVP inner layer fibers. Fibers were loaded with 1, 2, 10 and $20 \% \mathrm{w} / \mathrm{w} \mathrm{NP} / \mathrm{fiber}$, resulting in high NP incorporation, regardless of and corresponding to, theoretical loading (Table 5.2). The encapsulation efficiency of maximally-loaded NP-fiber composites $(20 \% \mathrm{w} / \mathrm{w})$ was 97, 65, and $46 \%$ for PEO, PVA, and PVP, respectively. Based on these results, PEO was selected as the hydrophilic layer for the multilayered composite. 
Table 5.2. Coumarin-6 NP loading in PEO, PVA, and PVP NP-EF composites. Dilutions of NP-EF composites, dissolved in DMSO, incorporating 10, 20, 100, or $200 \mu \mathrm{g} / \mathrm{mg}$ C6 NPs were evaluated by measuring fluorescence. Three composite batches were fabricated for each formulation, with $\mathrm{C} 6$ loading expressed as mean \pm standard deviation of triplicate readings of each NP batch. Statistical significance was assessed using one-way ANOVA with the Bonferroni post hoc test.

\begin{tabular}{|c|c|c|c|}
\hline \multirow{2}{*}{ Fiber Formulation } & $\begin{array}{c}\text { Theore tical Loading } \\
\mathbf{N P / E F}(\boldsymbol{\mu g} / \mathbf{m g})\end{array}$ & $\begin{array}{c}\text { Actual Loading } \\
\mathbf{N P / E F}(\boldsymbol{\mu g} / \mathbf{m g})\end{array}$ & $\begin{array}{c}\text { Encapsulation } \\
\text { Efficiency }(\boldsymbol{\%})\end{array}$ \\
\hline \multirow{3}{*}{ PEO } & 10 & $10.5 \pm 1.2$ & $105 \pm 11$ \\
\cline { 2 - 4 } & 20 & $15.8 \pm 1.2$ & $78.9 \pm 3.9$ \\
\cline { 2 - 4 } & 100 & $77.6 \pm 23.0$ & $77.6 \pm 23.0$ \\
\cline { 2 - 4 } & 200 & $194.6 \pm 11.3$ & $97.3 \pm 6.0$ \\
\hline \multirow{3}{*}{ PVA } & 10 & $7.4 \pm 0.5$ & $74.3 \pm 4.3$ \\
\cline { 2 - 4 } & 20 & $13.4 \pm 1.2$ & $67.3 \pm 7.2$ \\
\cline { 2 - 4 } & 100 & $86.0 \pm 4.0$ & $86.8 \pm 4.9$ \\
\hline \multirow{3}{*}{ PVP } & 200 & $130.4 \pm 42.0$ & $65.2 \pm 22.4$ \\
\cline { 2 - 4 } & 10 & $9.1 \pm 1.1$ & $91 \pm 11.0$ \\
\cline { 2 - 4 } & 20 & $9.2 \pm 1.1$ & $46.0 \pm 6.2$ \\
\cline { 2 - 4 } & 100 & $68.3 \pm 7.2$ & $68.3 \pm 7.2$ \\
\hline
\end{tabular}


Based on NP loading in the hydrophilic layer, multilayered PCL-PEO-PCL composites that incorporated 1 or $20 \% \mathrm{w} / \mathrm{w}$ mPEG-PLGA GRFT NPs $(200 \mu \mathrm{g} / \mathrm{mg}$ GRFT) were fabricated. As shown in Figure 5.4, both PEO (inner) and PCL (outer) layers maintained fiber morphology and integrity. Subsequent loading studies showed that $58 \pm 8$ and $56 \pm 8 \%$ of GRFT NPs were incorporated from 1 and $20 \%$ NP w/w loaded multilayered NP-EFs. 

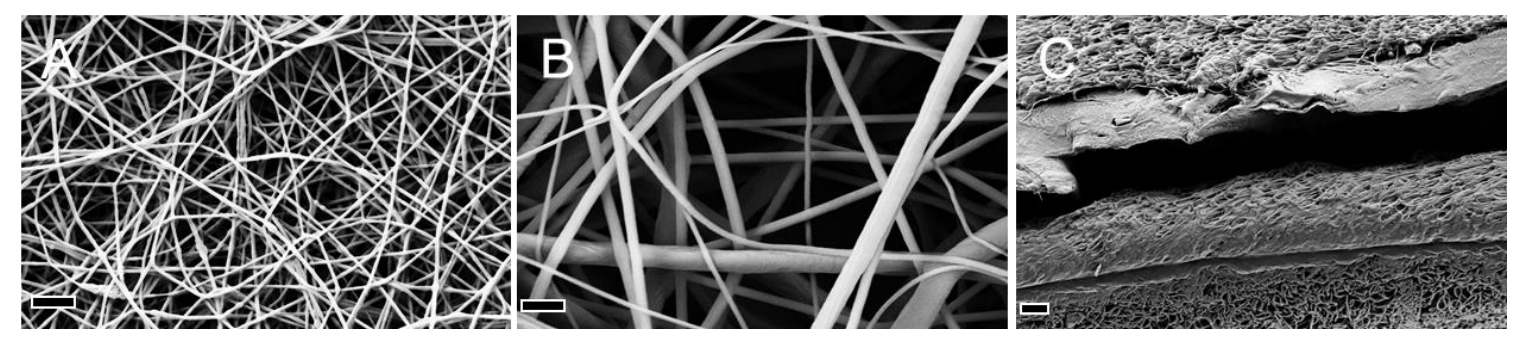

Figure 5.4. Scanning electron microscopy images of GRFT NP-EF composites taken from $(A)$ inner layer PEO fibers $(5 \% \mathrm{w} / \mathrm{w})$ that incorporate $20 \% \mathrm{w} / \mathrm{w}$ blank mPEG-PLGA NPs, (B) outer layer PCL fibers, and (C) a cross-sectional image of an NP-EF composite. Scale bars for panels A and B represent $2 \mu \mathrm{m}$, while the scale bar in panel $\mathrm{C}$ represents $20 \mu \mathrm{m}$. 
Sustained-release studies were conducted to assess GRFT release from NP-EF composites with varying outer layer thicknesses corresponding to 1, 3, 5 and $7 \mathrm{~mL}$ volumes. First, release was measured from composites that contained 1\% w/w GRFT NPs (Figure 5.5A, B). All formulations, regardless of thickness, exhibited an initial burst release through $\sim 72 \mathrm{hr}$, followed by sustained-release of GRFT for up to $90 \mathrm{~d}$. Overall, increasing the PCL outer layer thickness resulted in decreased GRFT release. After $90 \mathrm{~d}$, the total GRFT released from fibers was 81, 67,54 , and $57 \mathrm{ng} / \mathrm{mg}$ for $1,3,5$ and $7 \mathrm{~mL}$ composites respectively. 

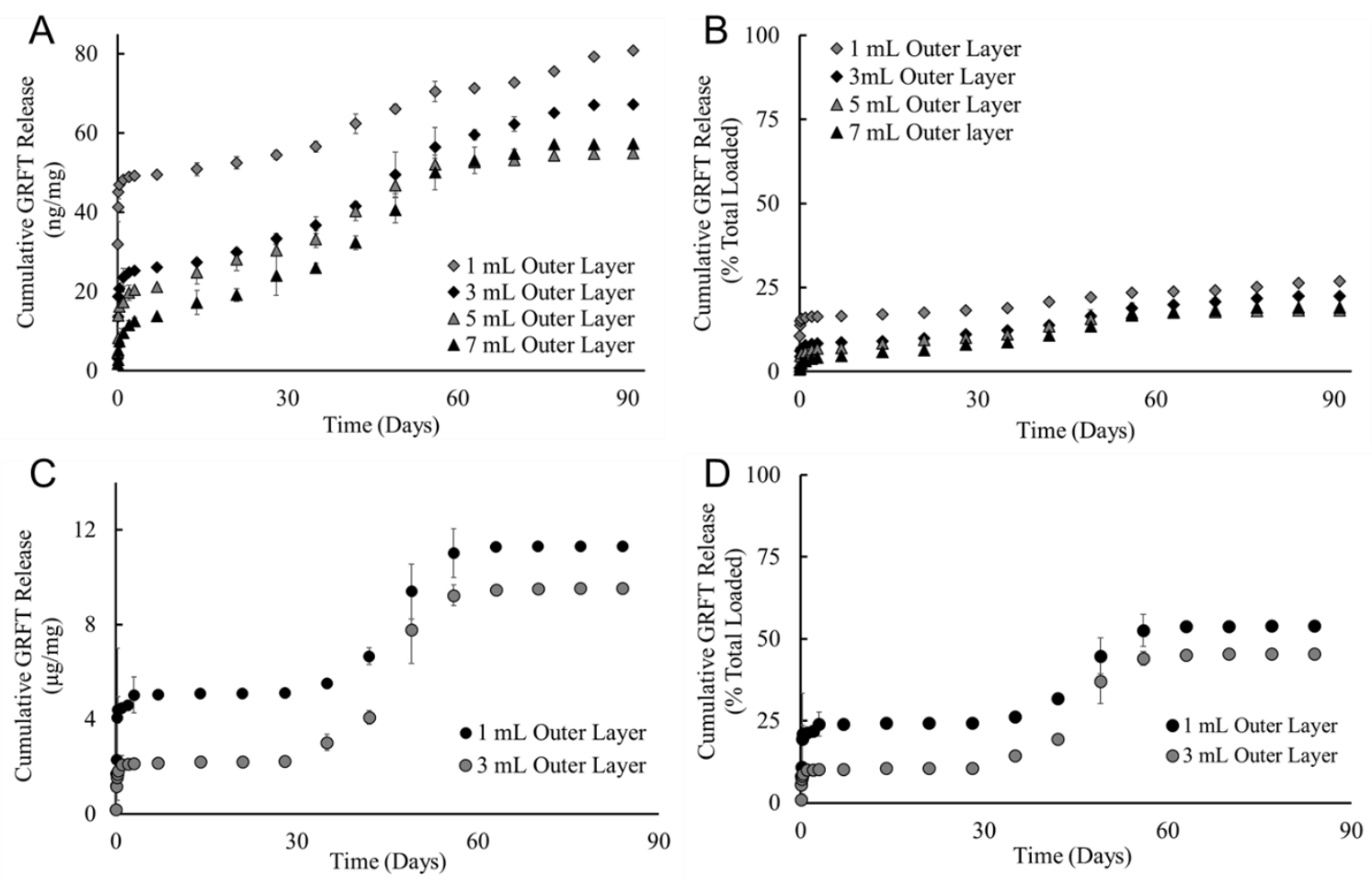

Figure 5.5. Cumulative release of GRFT from multilayered GRFT NP-EF composites. The cumulative release of GRFT from multilayered mPEG-PLGA NPEF composites formulated with $1 \%$ w/w NP loading, with different outer layer thicknesses shown as (A) total GRFT release or (B) percent of total loading. Subsequent release studies evaluated the cumulative release of GRFT from mPEG-PLGA NP-EF composites formulated with $20 \% \mathrm{w} / \mathrm{w}$ NPs shown as (C) total GRFT release or (D) percent of total loading. Overall, GRFT release increased from composites with thinner shells. Griffithsin release values are shown as the mean \pm standard deviation of three independent NP-EF batches. 
Based on the release results, composites that incorporated 20\% w/w GRFT NPs with 1 and $3 \mathrm{~mL}$ PCL outer layers were fabricated. After $90 \mathrm{~d}$, the total GRFT released was 11 and $9 \mu \mathrm{g} / \mathrm{mg}$ (53 and $45 \%$ total release) for the 1 and $3 \mathrm{~mL} \mathrm{NP}$ EF composites (Figure5. 5C, D). Release profiles showed similar trends to $1 \%$ NP-EFs in that there was a noticeable burst release during the first $72 \mathrm{hr}$; however, decreased release was observed through week 4, while increased GRFT release was observed between weeks 4 and 8 .

\section{In Vitro Viral Inhibition Studies}

Both mPEG-PLGA GRFT NPs and NP-EF composites were assessed in vitro against HIV-1 and HSV-2 infections. Dilutions of GRFT NPs and eluates from $20 \% \mathrm{w} / \mathrm{w}$ NP-EF composites were administered to TZM-bl cells 1 and $24 \mathrm{hr}$ prior to viral infection. GRFT NPs demonstrated complete dose-dependent inhibition against HIV-1 at both time points (Figure 5.6A, B). Furthermore, the $\mathrm{IC}_{50}$ of GRFT released from NPs was comparable to free GRFT. Additionally, GRFT released from NP-EF composites demonstrated comparable efficacy relative to free GRFT, achieving complete protection (> 98\%) after 1 and $24 \mathrm{hr}$ administration. The $\mathrm{IC}_{50}$ values of GRFT NPs, GRFT NP-EFs, and free GRFT were $51.1 \pm 2.3,35.6 \pm 7.1$, $21.5 \pm 5.3$, after $1 \mathrm{hr}$ and $36.1 \pm 6.8,29.7 \pm 18.1$, and $20.8 \pm 6.7 \mathrm{ng} / \mathrm{mL}$ after $24 \mathrm{hr}$ administration. All platforms demonstrated statistically similar $I_{50}$ values to free GRFT, with the exception of $1 \mathrm{hr}$ GRFT NPs, which showed a statistically higher $\mathrm{IC}_{50}$ value relative to both 1 and $24 \mathrm{hr}$ free GRFT. These results indicate that incorporation of GRFT in either NPs or NP-EFs composites maintains antiviral activity. 

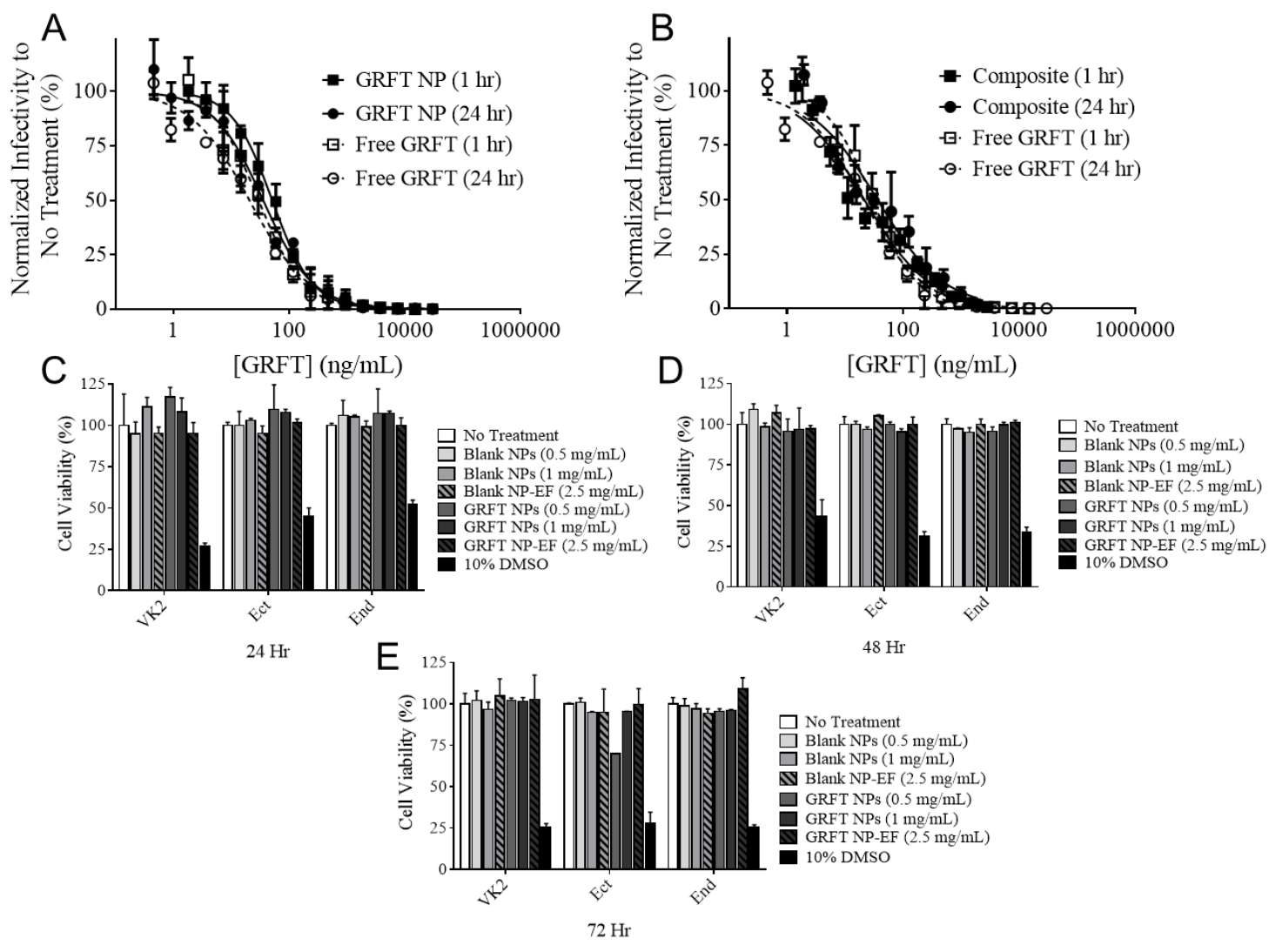

Figure 5.6. Results from in vitro HIV-1 inhibition and MTT toxicity assays. Three independent batches of GRFT NPs and NP-EF composites were assessed for their ability to inhibit HIV-1 infection. Nanoparticles or composite eluates were incubated with cells (A) $1 \mathrm{hr}$ and (B) $24 \mathrm{hr}$ prior to HIV-1 infection. GRFT released from both platforms exhibited complete HIV-1 inhibition at both 1 and $24 \mathrm{hr}$, relative to free GRFT. The cytotoxicity of the maximum dose of GRFT NPs and NP-EFs used in the in vitro assays was administered to vaginal VK2/E6E7, Ect1/E6E7, and End1/E6E7 cells for (C) 24, (D) 48, and (E) $72 \mathrm{hr}$ was assessed using the MTT assay. Greater than $94 \%$ viability was observed in all cell lines for all NPs and NPEF formulations. 


\section{In Vitro Safety Studies}

Prior to in vivo studies, both blank and GRFT NPs and NP-EFs were assessed for cytotoxicity in VK2/, Ect1/, and End1/E6E7 cells using MTT assays. All cell lines demonstrated greater than $94 \%$ viability after 24,48 , and $72 \mathrm{hr}$ incubation with samples, relative to untreated controls (Figure 5.6C-E).

\section{In Vivo Efficacy Studies}

The efficacy of GRFT NPs and GRFT NP-EF composites was assessed within a murine model of lethal HSV-2 infection. Delivery platforms were intravaginally administered to female BALB/c mice, followed by a single challenge of HSV-2 (LD90, 5,000 PFU) 24 hr later (Fig. 5.7A, B, Table 5.3). Mice were monitored for $14 \mathrm{~d}$ post-infection, and Kaplan-Meier survival analyses were applied to generate survival curves (Fig. 5.7C). Mice administered GRFT NPs or GRFT NP-EF composites showed statistically significant decreases in HSV-2 infection (70 and $80 \%$ survivability, respectively), relative to the untreated/infected control group ( $5 \%$ survivability, $p<0.05)$. Additionally, GRFT NPs exhibited statistically similar protection to free GRFT (85\% survivability), whereas GRFT NP-EFs demonstrated a slight decrease in protection, relative to free GRFT controls $(p<$ 0.05). Additionally, the administration of blank NPs provided a significant level of protection against HSV-2 (35\% survival), relative to GRFT NPs, GRFT NP-EFs, and untreated/infected controls $(p<0.05)$. 
A

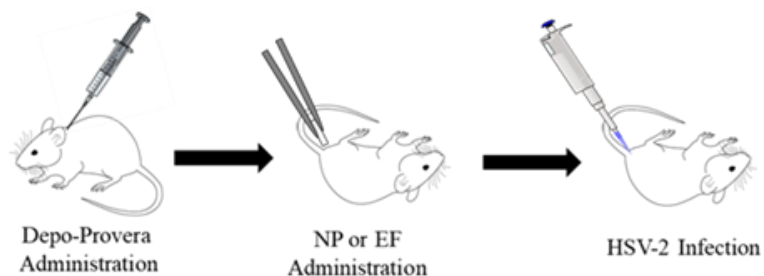

B

Efficacy Studies
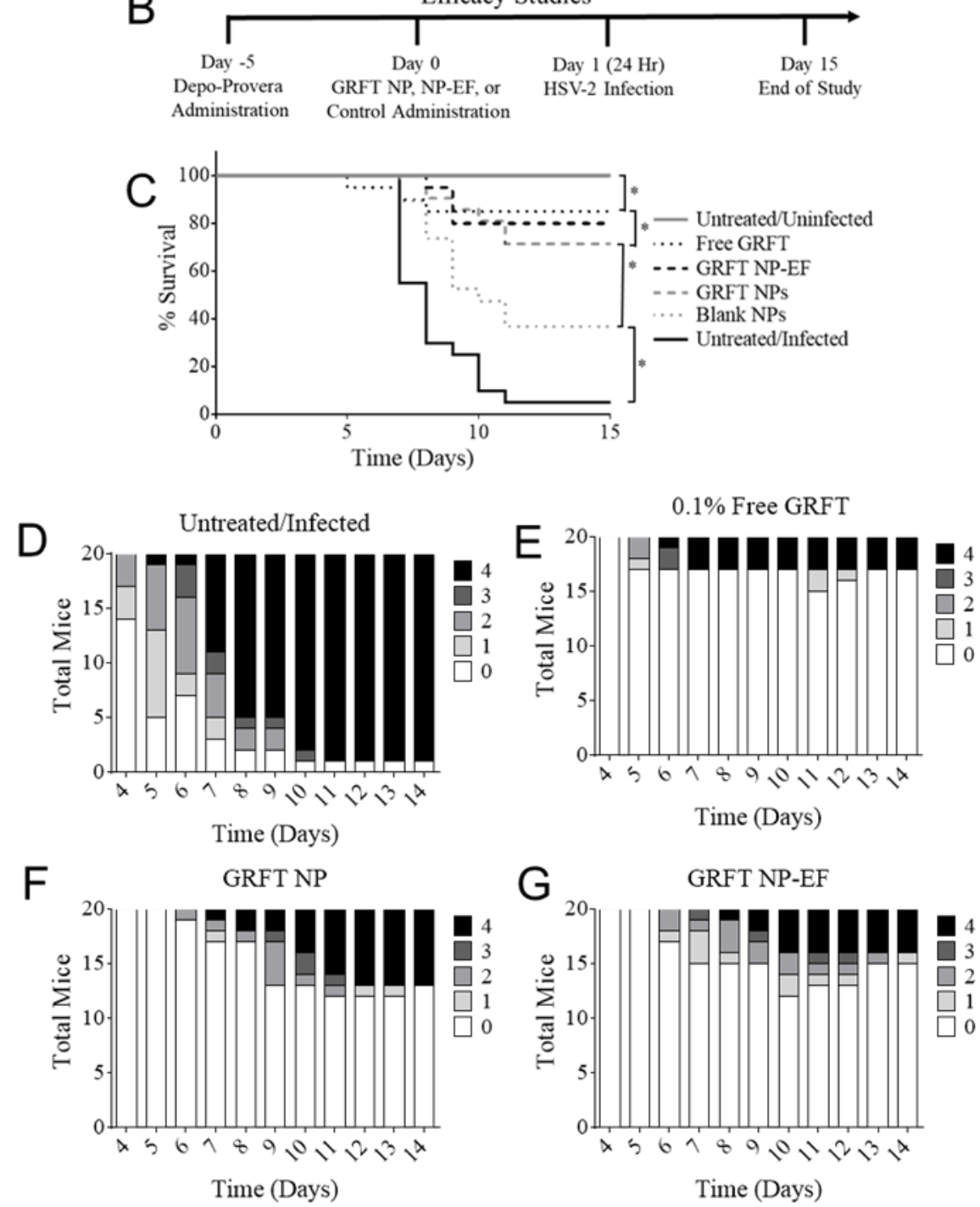

Figure 5.7. Schematic, timetable, and results of in vivo HSV-2 efficacy study. (A) Sequence of murine treatments and (B) administration schedule from HSV-2 efficacy study. (C) Kaplan-Meier survival curves from HSV-2 in vivo efficacy study. GRFT platforms were assessed for their ability to protect mice against a lethal 
challenge (LD90) of HSV-2 infection $(n=20)$. Both GRFT NPs and NP-EF composites demonstrated comparable protection (70\% and $80 \%$, respectively) relative to free GRFT (85\%), and demonstrated a significant increase in protection relative to untreated/infected controls ( $5 \%$ survival). Additionally, blank NPs were found to decrease viral infection and protect $35 \%$ mice after $14 d,{ }^{*} p<0.05$. Furthermore, mice were evaluated and scored for progression of infection once daily for $14 \mathrm{~d}$ post-infection. Infected mice administered (D) no treatment, $(\mathrm{E})$ free GRFT, (F) GRFT NPs or (G) GRFT NP-EFs exhibited decreased severity of infection and more gradual progression of infection, relative to untreated/infected animals. 
Table 5.3. Dosing regimen of GRFT NPs, GRFT NP-EFs, and free GRFT administered in in vivo studies.

\begin{tabular}{|c|c|c|}
\hline Formulation Name & $\begin{array}{c}\text { Amount of Vehicle } \\
\text { Administered per Mouse }\end{array}$ & $\begin{array}{c}\text { Total GRFT Administered } \\
\text { Based on Loading ( } \boldsymbol{\mu} \text { ) }\end{array}$ \\
\hline GRFT NPs & $20 \mu \mathrm{L}$ of $25 \mathrm{mg} / \mathrm{mL}$ PBS & 63 \\
\hline GRFT NP-EFs & $5 \mathrm{mg}$ & 71 \\
\hline $0.1 \%$ w/v GRFT Solution & $20 \mu \mathrm{L}$ in PBS & 20 \\
\hline
\end{tabular}


In addition to preventing HSV-2 infection, the mice treated with GRFT NPs, GRFT NP-EF composites, or free GRFT that became symptomatic, demonstrated delayed onset of symptoms and a more gradual progression of infection, relative to the untreated/infected control group (Figure 5.7D-G). In the untreated/infected control group, the first symptoms of HSV-2 infection typically manifest 4 to $5 \mathrm{~d}$ postinfection, during which time mice exhibit localized vaginal swelling and decreased hind leg mobility. After 5 to $8 \mathrm{~d}, \sim 75 \%$ of mice from the untreated/infected control group required euthanasia due to the rapid progression and severity of infection. In contrast, mice exposed to free GRFT or GRFT delivery platforms showed decreased progression of symptoms within the same duration. Mice that did not survive HSV-2 challenge despite GRFT pre-treatment, exhibited a more gradual progression of infection, requiring euthanasia 7 to $10 \mathrm{~d}$ instead of 5 to $8 \mathrm{~d}$ postinfection, suggesting that GRFT may provide partial protection at these doses for the few mice that exhibited overt signs of infection. No differences were observed in the progression of HSV-2 infection after administration of blank NPs, relative to the untreated/uninfected control group (data not shown).

\section{In Vivo Safety Studies}

To assess the in vivo safety of these delivery platforms, blank NPs and NPEF composites were intravaginally administered to uninfected mice, and reproductive tissue and vaginal lavages were collected and analyzed 24 and $72 \mathrm{hr}$ following administration. 
Tissue samples were evaluated for edema of muscle, interstitial, and epithelial tissue. In addition, the macrostructure of untreated and vehicle-treated cervical and vaginal epithelia were compared, and assessed for potential keratinization and goblet cell presence. A score ranging from 1 to 4 was used to determine the severity of epithelial changes. Images of tissues collected $24 \mathrm{hr}$ after vehicle administration are shown in Figure 5.8A-E. Extracted tissues from untreated controls demonstrated compact squamous and cervical epithelial tissue, with no evidence of edema nor inflammation. Similarly, tissues treated with blank NPs or NP-EF composites showed similar morphology and normality, relative to untreated tissue (scores of 1 to 2). Furthermore, no increase in lymphocyte accumulation was observed in these groups. Overall the tissues from vehicletreated mice were comparable in morphology to tissues from untreated mice. 


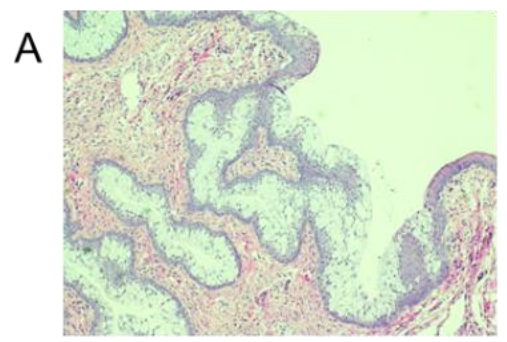

Untreated
B

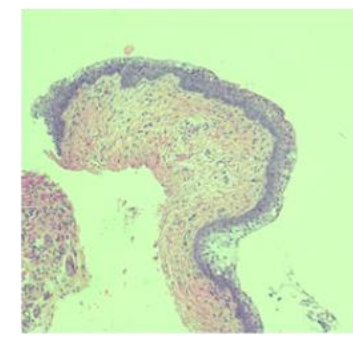

Sham Control

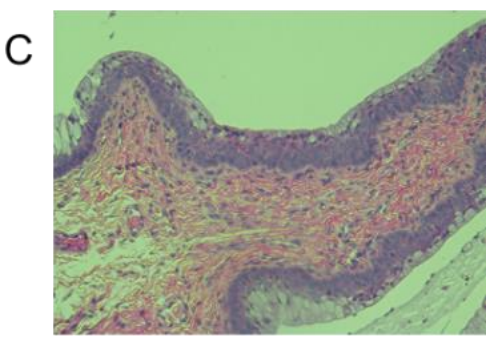

$4 \%$ N-9 Gel

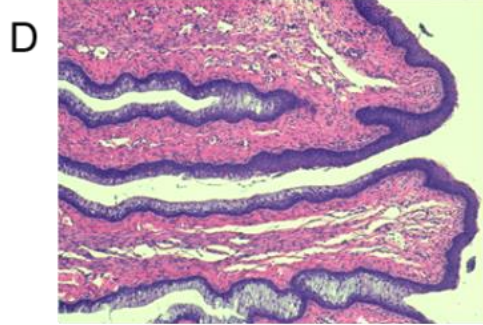

Blank NPs
E

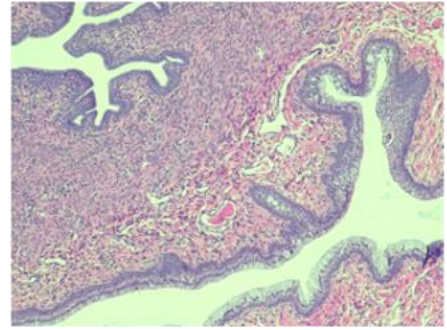

Blank NP-EFs
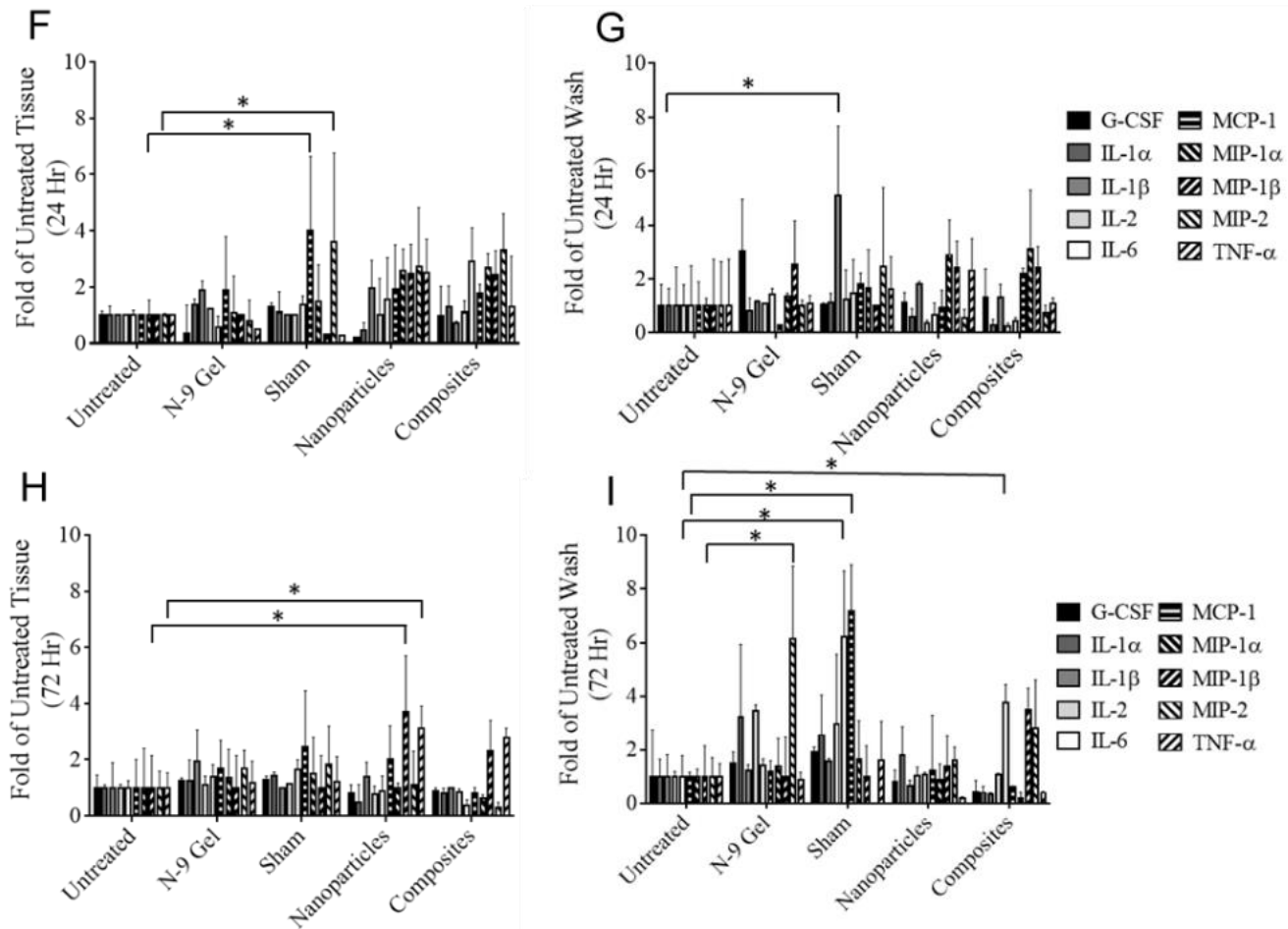

Figure 5.8. The safety of NPs and NP-EF composites was assessed by administering platforms to murine reproductive tracts for 24 and $72 \mathrm{hr}$. H\&E stained tissues of murine reproductive tracts exposed to (A) no treatment, (B) shamtreatment, (C) blank NPs, and (D) blank NP-EF composites for $24 \mathrm{hr}$. No indication 
of tissue inflammation or epithelial disruption was observed after NP or NP-EF administration, relative to untreated controls. Additionally, cytokine expression was evaluated from extracted murine tissue and vaginal lavages collected (E, F) 24 and (G, H) $72 \mathrm{hr}$ after vehicle administration. Cytokine expression was assessed using previously published criteria, with acceptable safety values within a 2 to 5 -fold range of expression in the untreated control group. Statistical analysis was used in addition to evaluate possible increased cytokine expression in both tissue and vaginal lavage samples. Mice treated with blank NPs or NP-EF composites expressed similar cytokine levels relative to untreated controls, with no cytokine levels demonstrating over 5-fold increase relative to untreated controls, indicating that the presence of either platform does not induce inflammation or immune response. Statistical significance between untreated controls and other groups represented by * $(p<0.05)$. 
For N-9 treated mice, a slight increase in neutrophil accumulation was observed at the surface of the cervical squamous epithelium, relative to untreated controls, indicating minor topical damage (score range 1 to 2). One N-9 treated sample exhibited increased inflammation, due to the presence of peripheral blood mononuclear cells, goblet cell fusion, and epithelial disruption, resulting in a score of 3 (data not shown). However, the alterations observed in the $\mathrm{N}-9$ control replicate were not observed in any $72 \mathrm{hr} \mathrm{N}-9$ treated tissue controls, indicating transitory damage.

Finally, cytokine expression was assessed in collected murine tissues and vaginal lavages 24 and $72 \mathrm{hr}$ after blank NP and NP-EF administration. Cytokine expression from blank NP or NP-EF treated mice was compared to levels in untreated mice, as well as to N-9 and sham-treated control groups. Cytokine expression was evaluated based on previously published guidelines, in which a significant level of intravaginal inflammation results in a 2 to 5 -fold increase in cytokine expression, relative to untreated controls ${ }^{69}$. Cytokine expression from extracted vaginal tissue and vaginal lavages is shown in Figure 5.8F-I, indicating that both blank NPs and NP-EFs are non-inflammatory. In both collected vaginal tissue and lavage samples treated with blank NPs and NP-EF composites, cytokine levels were comparable to untreated negative controls $(p>0.05)$. This trend was exhibited both 24 and $72 \mathrm{hr}$ post-administration, with no statistical significance in cytokine expression between most untreated and blank fibertreated groups. The only exceptions observed were in tissues that had been administered NPs for $72 \mathrm{hr}$, which demonstrated decreased cytokine levels of both 
MIP-1 $\beta$ and TNF- $\alpha$, and $72 \mathrm{hr}$ wash samples from the NP-EF treated group, which showed increased IL-6 expression, relative to the untreated group. For shamtreated controls, which simulated the administration method via tweezers only, increased cytokine expression was observed in $24 \mathrm{hr}$ tissue (MIP-2), $24 \mathrm{hr}$ wash (IL-1ß), and $72 \mathrm{hr}$ wash (IL-6, MCP-1), relative to untreated controls. The N-9 treated group demonstrated an increase of MIP-2 in $72 \mathrm{hr}$ wash samples only. Increased TNF- $\alpha$ expression is typically associated with increased IL-1 $\alpha$, IL-1 $1 \beta$, and IL-2 expression, whereas increased MIP-1 $\beta$ is associated with increased neutrophil accumulation, neither of which were observed in NP-treated tissue samples $337,338,340$. Additionally, increased IL-6 expression alone is not indicative of inflammation, but is overexpressed with TNF- $\alpha$ and IL-1 $\alpha$ under conditions of localized inflammation, which were not observed in NP-EF treated mice ${ }^{356}$. Therefore, we attribute the increased expression of IL-6, MIP-1 $\beta$ and TNF- $\alpha$ to variability in samples, and not inflammation, as our delivery platforms did not elicit

increased the expression of multiple cytokines. An ELISA, used to confirm IL-1 $\beta$ levels, showed similar results, showing that both blank NP and NP-EF composites induced negligible increases in cytokine expression, relative to untreated controls $(p>0.05)$.

\section{Discussion}

During the past decade, Griffithsin has been studied for its activity against a variety of viruses, including HSV-2 and HIV-163,65,66, and has demonstrated an outstanding in vivo safety profiles, with no induction of inflammatory or cytokine responses. Given these successes, recent work has focused on exploring GRFT 
delivery in topical dosage forms to provide on-demand delivery options in preventing viral infections. Toward these efforts, recent work evaluating GRFT gels has shown in vivo efficacy against both HIV-1 and HSV-2. Moreover, other studies demonstrated the efficacy of GRFT fast dissolving inserts (FDIs) in vivo ${ }^{73,74}$. In these studies, both gels and inserts released GRFT within $4 \mathrm{hr}$ to protect mice against a lethal dose of HSV-2 infection ${ }^{65,73}$. Furthermore, previous work in our group has developed $\mathrm{pH}$-responsive and surface-modified fibers that incorporate GRFT for short-term protection against HIV-1 infections ${ }^{75,162}$. While these technologies may provide on-demand protection, to date, vehicles have not been developed that provide long-term (here, $>72 \mathrm{hr}$ ) biologic or GRFT delivery options. Delivery platforms that provide sustained-release may enable less frequent administration to provide an effective and practical long-term prevention strategy that is less reliant on user adherence.

To achieve long-term prevention, multilayered nanoparticle-fiber composites were developed in this study to provide a dual-purpose sustainedrelease platform against HSV-2 and HIV-1 infections. Previous studies for other applications (e.g., chemotherapy, antibacterial) have demonstrated the potential of multilayered fibers alone to provide spatially-specific release, where specific layers incorporate active agents, and other layers are tailored to delay or tune release ${ }^{177-182,184,357}$. These architectures provide unique advantages relative to traditional uniaxial fiber architectures, particularly for sustained-release applications. Multilayered fibers comprised of distinct hydrophilic and hydrophobic polymer layers have combined the advantages of both polymer types to highly 
incorporate small hydrophilic drugs or biologics, resulting in better tunability of release $^{357}$. Given these attributes, parallel to these NP-EF composite studies, free GRFT was incorporated into multilayered fibers with shell thicknesses similar to the NP-EF composites. Although shell thickness did delay release from multilayered fibers, complete GRFT release from all fibers was observed within 72 hr (Supplemental Fig 5.2), which may be attributed to the pore sizes of PCL shell layers and the lack of NPs to further modulate release (Figure 5.4B). 

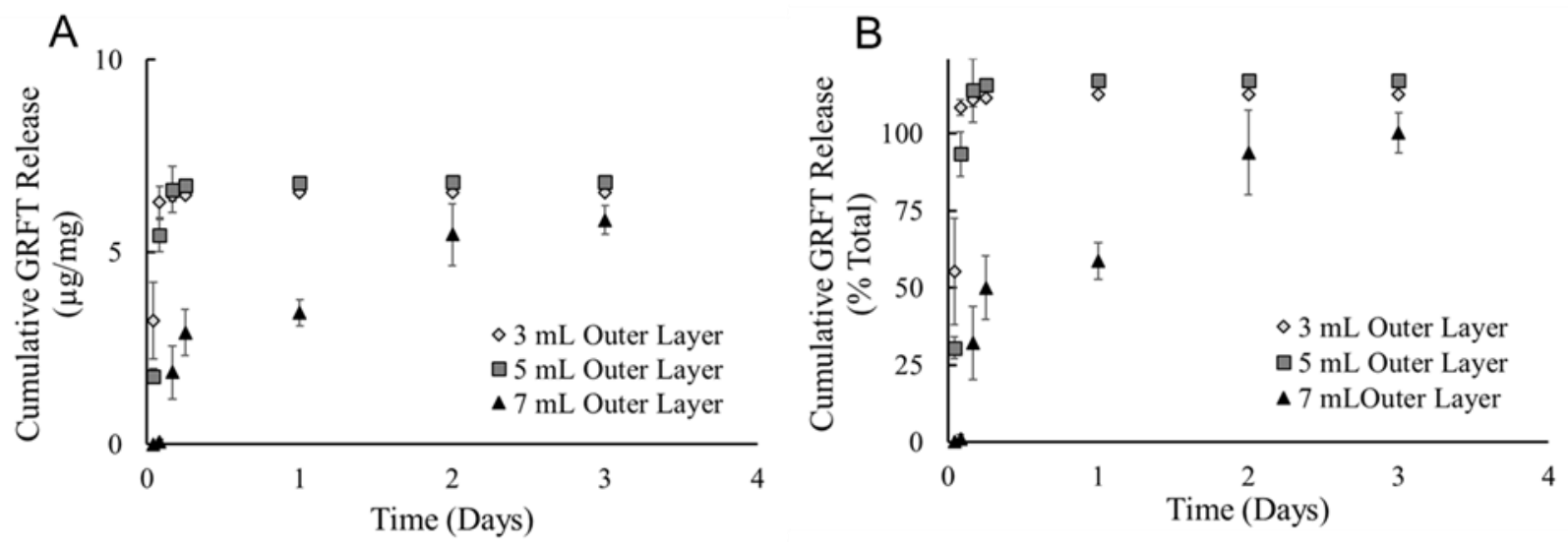

Supplemental Figure 5.2. The cumulative release of GRFT from multilayered fibers with different outer layer thicknesses shown as (A) total GRFT release or (B) percent of total loading. Increased GRFT release was observed from multilayers with thinner shells, although complete release was observed in all samples after $72 \mathrm{hr}$. Griffithsin release values are shown as the mean \pm standard deviation of three independent NP-EF batches. 
While multilayered electrospun fibers alone (without NPs) have been previously studied to provide the sustained-release of hydrophilic agents ${ }^{177-182,184}$, NP-EF composites have been less extensively studied to prolong delivery ${ }^{198,206-}$ 209. In one proof-of-concept study, multilayered fibers alone comprised of a PCL shell and a PEO inner layer were fabricated to provide sustained-release of the small hydrophilic dye rhodamine B for up to 15 days $^{358}$. By increasing the shell thickness by a factor of 4 , release was extended up to 25 days, with thicker outer layers providing close to zero-order release profiles, relative to fibers with thinner outer layers. More recently, hydrophilic, rapid-dissolve NP-EF single-layered composites have been designed to enhance agent retention within the murine reproductive tract ${ }^{215}$. Hydrophilic PVA and PVP fibers incorporating rhodamine $B$ or etravirine PLGA NPs were fabricated and intravaginally administered to mice. It was found that composite dissolution within murine tissue was slower, relative to in vitro release studies, due to the decreased wettability within the murine reproductive tract, which contributed to the prolonged release of both rhodamine B and etravirine. The intravaginal retention of rhodamine was 30 -fold higher $24 \mathrm{hr}$ after composite administration ( $\sim 50 \%$ total dose present) relative to administration of free NPs (1.5\% total dose), in addition, detectable amounts of rhodamine were present within the reproductive tract for up to $72 \mathrm{hr}$. Additionally, etravirine released from NP-EF composites demonstrated a 12-fold increase in concentration after 72 hr composite administration (44\% total dose), relative to free NP administration (1.2\% total dose). For both rhodamine B or etravirine PLGA NPs, durations longer than $24 \mathrm{hr}$ post-administration showed a significant fraction of freely administered 
NPs ( 99\%) absent from the murine reproductive tract, due to high mucus clearance. In contrast, NP-EF composites provided short-term release of both compounds and improved rhodamine and etravirine retention over the course of 72 and $120 \mathrm{hr}$, respectively. Although this study demonstrated only short-term release (up to $120 \mathrm{hr}$ ) using readily dissolvable NP-EF composites, it demonstrates the potential of nanoparticle-fiber composites to prolong active agent release, relative to the administration of free NPs. While NP encapsulation within hydrophilic polymers such as PVA and PVP may improve NP retention for durations less than one week, we hypothesized that the incorporation of hydrophobic polymers in more complex multilayered architectures may significantly extend release profiles of active agents compared to using to fibers or NPs alone.

In this work, combining the unique advantages of electrospun fibers and nanoparticles, resulted in extended GRFT release (up to $90 \mathrm{~d}$ ) from multilayered nanoparticle-fiber composites, relative to PLGA and mPEG-PLGA NPs, which released GRFT within one week. GRFT NP-EFs demonstrated long-term release, particularly from the formulation that incorporated a lower concentration of NPs (1\% w/w). Based on these results, GRFT NP-EF composites incorporating $20 \%$ w/w GRFT NPs were fabricated and assessed for sustained-release. Although a greater amount of GRFT release was demonstrated in the $20 \% \mathrm{w} / \mathrm{w}$ composites, compared to $1 \%$ NP-EFs, higher burst release was observed in the $20 \% \mathrm{w} / \mathrm{w}$ composites, with negligible release between weeks 1 and 4 . However, both 1 and $20 \%$ formulations demonstrated extended GRFT release from wk 5 through wk 9 , 
which was attributed to NP and EF degradation. Future studies may evaluate other polymer types or polymer blends in the outer layer to further tailor GRFT sustainedrelease profiles. Additionally, outer layer thickness provided some modulation of GRFT release, but additional options such as electrospinning conditions affecting fiber micromorphology such as pore size, may provide further tunability of release. Moreover, the overall multilayered fiber architecture, including the number of discrete fiber layers, may be changed to further adjust GRFT release. Finally, the differences between in vitro models of release and the reproductive tract must be considered as well. The decreased wettability within the FRT, may prolong release of agents from composites, to in vitro models ${ }^{215}$.

In addition, to addressing the need for sustained-release delivery, another benefit of composite platforms is that both NPs and fibers can preserve the activity of encapsulants. While GRFT has been shown to retain activity in a variety of physiological environments, including human semen and blood $68,73,162,359$, studies have shown that GRFT is susceptible to oxidation, specifically the $78^{\text {th }}$ amino acid residue (methionine), which may impact its long-term stability ${ }^{360,361}$. Moreover, studies evaluating GRFT-containing FDIs showed that GRFT was initially susceptible to thermal shear and compression stresses, in addition to oxidation during conventional FDI fabrication processes, requiring specialized fabrication in order to provide stability for incorporated $\mathrm{GRFT}^{74}$. Similar to these specialized FDIs, electrospun fibers have been shown to preserve the stability of incorporated proteins ${ }^{80,362,363}$. In particular, previous work has shown that incorporated agents released from fibers provided enhanced long-term in vitro efficacy relative to free 
agents, due to fibers preserving encapsulant activity ${ }^{357}$. Moreover, the incorporation of hydrophilic polymers can improve biologic encapsulation and maintain protein integrity and activity, relative to more hydrophobic polymers that often require harsh organic solvents during fabrication.

In line with previous observations regarding NPs and fibers, multilayered NP-EFs were also shown to preserve the activity of incorporated GRFT. The results from in vitro HIV-1 inhibition assays demonstrated that GRFT released from either NPs or NP-EF composites provided comparable antiviral activity relative to free GRFT. These results are in agreement with previous studies that evaluated free GRFT and GRFT incorporating pH-responsive and surface-modified fibers, demonstrating a high degree of stability and retention of functional activity ${ }^{75,162}$. Similar results are shown here, suggesting that multilayered composites maintain the structure and corresponding functional activity of GRFT. More importantly, composites provided similar inhibition of in vivo HSV-2 infections relative to free GRFT. Future work with composites seek to optimize GRFT and GRFT NP loading, and will potentially evaluate the co-delivery of multiple active agents with different mechanisms of action, to reduce doses required to provide dualprotection.

While this study sought to translate the antiviral properties of GRFT NPs and composites to in vivo applications, GRFT NPs and NP-EF composites were initially evaluated using an HSV-2 challenge model $24 \mathrm{hr}$ following vehicle administration. This challenge time was selected to evaluate the initial potential of these vehicles to provide complete protection relative to other GRFT-based on- 
demand platforms currently under evaluation. Both GRFT NPs as well as GRFT composites provided potent protection against infection, similar to free GRFT. Moreover, for the few mice that were infected, GRFT reduced the infection severity and disease course, which is in line with previous studies that evaluated the in vivo efficacy of GRFT gels ${ }^{62}$. Another observation from the efficacy study was that blank platforms provided modest levels of protection against viral infection relative to untreated/infected controls ( $35 \%$ survivability). This confirms previous in vitro studies from our group that have shown that polymer presence alone can serve as a physical barrier to virus infection ${ }^{75,87}$. In future work, we may evaluate different shapes and sizes of composites, in line with a dose-optimization study to provide a more detailed characterization of this delivery platform.

In addition to investigating dose response and evaluating the potential of composites to act as a physical barrier of protection, intravaginal retention active agents released from topical delivery vehicles could be explored further. In other studies that evaluated GRFT and carrageenan gels, efficacious GRFT concentrations were detected in murine models $8 \mathrm{hr}$ following initial administration, despite in vitro FDI dissolution occurring within $1 \min ^{73}$. These studies attributed epithelial-bound GRFT to the high concentrations present for hours after vehicle dissolution. In addition to the decreased wettability of the delivery platforms presented here, we hypothesize that GRFT and even vehicle binding may further extend the GRFT retention within the reproductive tract. Although examining the pharmacokinetics of GRFT released from multilayered composites in murine reproductive tissue was beyond the scope of this work, future studies will explore 
how intravaginal retention relates to the long-term protection imparted by multilayered composites against single or multiple challenges of HSV-2 at different times with respect to composite administration. Based on the release studies presented here, we anticipate that GRFT composites will maintain efficacious levels of GRFT on the order of weeks to months, depending on fiber dose and NP loading, and will demonstrate long-term efficacy against both HIV-1 and HSV-2 infections.

Finally, the safety of nanoparticles and multilayered composites were assessed in both in vitro and murine models. These safety studies were conducted to evaluate potential toxicity or inflammation caused by the administration of these delivery platforms, representing an important criteria of topical delivery platforms. Previous work has shown that increased cytokine expression within the female reproductive tract is associated with an increased risk of HIV-1 acquisition, which may negate any protective attributes of a delivery platform ${ }^{364}$. Results from cytotoxicity and histology studies showed that blank and GRFT NPs and NP-EF composites demonstrated safety and biocompatibility. Furthermore, cytokine expression levels from vaginal lavages and tissues exposed to NP and NP-EFs were within a 2 to 5 -fold range of levels in untreated controls, indicating the preliminary safety of these delivery platforms ${ }^{69}$. These results were anticipated, given the established safety profiles of the selected polymers, the encapsulant GRFT, and our previous observations with electrospun fibers and NPs individually.

\section{Conclusion}


In this study, multilayered NP-EF composites that incorporate the antiviral lectin GRFT were fabricated to provide dual-protection against HIV-1 and HSV-2 infections. Composites demonstrated high loading of GRFT NPs and achieved sustained-release of GRFT, over a duration of $90 \mathrm{~d}$, dependent on GRFT NP loading within the NP-EFs. Both NPs and NP-EF composites inhibited HIV-1 infection in vitro and moreover, these vehicles demonstrated protection against a lethal dose of HSV-2 infection in a murine model. Overall these platforms demonstrated preliminary safety and efficacy in vivo, suggesting the potential of these new composite platforms as an alternative sustained-delivery dosage form to provide dual-protection against HIV-1 and HSV-2 infections. 


\section{CHAPTER 6}

\section{OVERALL DISCUSSION AND CONCLUSION}

In these studies, electrospun fibers, composed of different polymer types and fiber architectures were fabricated to deliver the antiviral lectin GRFT or the antiretroviral TDF. The overall goal was to create topical delivery platforms that would provide dual-purpose protection against both HIV-1 and HSV-2 infections. Additionally, these platforms were designed to overcome challenges associated with other topical delivery platforms, such as transient release, as well as to provide an alternative dosage form for women in providing protection. Ultimately, the objective is to translate the success of these fiber formulations into effective dosage forms that provide efficacious, discreet, and convenient protection against STIs for women.

Studies have shown that HSV-2 infection increases the susceptibility to HIV1 infection by 2 to 4 -fold ${ }^{2,3}$. Hence, there is a pressing need to provide dualpurpose protection against both HIV-1 and HSV-2 infections. Additionally, women are disproportionately impacted by the HIV pandemic, necessitating strategies focused on new alternatives for female-applied protection ${ }^{365}$.

The development of active agents that provide multipurpose protection against HIV-1 and HSV-2 is a potential solution. There are dozens of therapeutics 
available to treat either HIV-1 or HSV-2; however only a fraction of these have the ability to prevent both infections ${ }^{55,366}$. Currently, Truvada ${ }^{\circledR}$ is the only oral tablet FDA-approved to prevent HIV-1 infections ${ }^{27}$. One of the two active agents in the tablet, TDF, has shown activity against HSV-2, albeit with decreased efficacy relative to HIV-1. However, other traditional antivirals, such as valaciclovir, foscarnet, and raltegravir, also have the potential to provide dual-purpose protection ${ }^{367-369}$. However, these agents are concurrently used in treatment regimens, raising the risk of antiviral resistance and potentially rendering preventative strategies using these molecules ineffective.

Recently, new classes of antiviral biological agents have been discovered that possess potent antiviral activity. Polysaccharides including carrageenan or the proteins, of cyanovirin- $\mathrm{N}$ and scytovirin, have all shown promising activity against HIV-1300,370-372. However, the lectin GRFT, originally derived from a species of red algae has demonstrated potent activity within the picomolar range against HIV-1 and other viruses, relative to other biologic candidates ${ }^{66}$. Moreover, unlike other tested biologics, GRFT has been shown to be safe and biocompatible, and to induce negligible immune response or inflammation ${ }^{68,69}$.

Concurrent with the development of new active agents, efforts have focused on oral and topical pre-exposure prophylaxis (PrEP) to prevent viral infections. For oral PrEP, Truvada is the only oral tablet that is FDA-approved to prevent HIV-1 infection. However, efficacy is dependent on strict user adherence, and side effects have been reported with long-term use ${ }^{373,374}$. Additionally, many of the new antiviral biologics lack oral bioavailability, excluding their use for oral PrEP. Topical 
PrEP, where dosage forms are directly administered to the FRT, may reduce the occurrence of side effects relative to oral PrEP, and improve biologic delivery. However, the dosage forms commonly used to administer biologics, such as gels, films, or fast dissolving inserts, may be challenged with providing prolonged protection or achieving acceptable user adherence ${ }^{29}$. Several topical PrEP delivery platforms, such as gels and films, have showed disappointing results in clinical trials, due to low user adherence ${ }^{29,219,375}$. Although biologics such as GRFT have been successfully incorporated into gels and fast dissolving inserts to impart in vivo antiviral protection, to date there is no delivery platform that extend the release of GRFT within the FRT for longer than $72 \mathrm{hr} 65,73$.

In response to these challenges, this work sought to develop polymeric electrospun fibers to deliver TDF and GRFT for different temporal administration strategies. The wide diversity of polymers available for electrospinning, electrospinning parameters, and the different fiber architectures available allows for a variety of fibers types to be fabricated ${ }^{76,109,114,376}$. In these studies, fibers comprised of PLGA polymers were covalently surface-modified with GRFT, to act as a stationary viral trap. Results from in vitro HIV inhibition assays demonstrated that GRFT surface-modified fibers completely inhibited HIV-1, and that fibers alone reduced infection. Next, fibers composed of polymer blends were developed to provide $\mathrm{pH}$-responsive release of GRFT. In addition, fibers comprised of hydrophilic polymers were fabricated to provide rapid-release of GRFT against both HIV-1 and HSV-2 infections. All rapid-release formulations were shown to highly incorporate GRFT and to provide complete protection against both 
infections in vitro, while maintaining comparable in vivo protection relative to free GRFT. Finally, multilayered composites demonstrated the sustained-release of GRFT and corresponding potent antiviral activity against HSV-2 in vivo.

\section{Conclusion and Future Work}

During the course of these studies, target product profiles (TPP) were established for GRFT NPs, fibers, and NP-EF composites (Table A 1, Table A 2). These profiles defined the acceptable and ideal properties as criteria by which to measure these platforms, with for example, providing sustained-release of efficacious GRFT concentrations for at least one month. Other goals included providing in vitro anti-HIV-1 activity and in vivo safety and efficacy against HSV-2 infection. In these studies, the selected GRFT platforms provided complete in vitro inhibition of HIV-1 infection and more importantly, in vivo protection against HSV2 challenge at an LDgo dose. Furthermore, our in vivo safety studies confirm the preliminary safety of GRFT rapid-release EFs, NPs, and NP-EF composites, demonstrating the potential of these dosage forms for intravaginal application.

In accordance with the established TPPs, one of the first delivery criteria was that delivery vehicles would release GRFT concentrations that were efficacious against both HSV-2 and HIV-1 infections. Although these platforms failed to provide daily release of GRFT (600 ng/mL and $39 \mu \mathrm{g} / \mathrm{mL}$ for HIV -1 and HSV-2 respectively), these values were initially determined primarily from in vitro GRFT studies in completely preventing infections. Upon further evaluation, these values may not reflect the actual GRFT dose required to prevent infections in vivo, 
based on the efficacy of our delivery platforms assessed within HSV-2 murine models of infection. Despite the decreased GRFT release relative to initial TPP requirements, GRFT delivery platforms provided comparable protection against a LD90 of HSV-2 infection. In agreement with our observations, other studies that assessed GRFT FDIs demonstrated that GRFT is retained within the reproductive tract due to the interactions between the tissue and lectin ${ }^{73,74}$. Furthermore, it was found that efficacious concentration of GRFT would be retained for several days, lowering the required daily release of GRFT. Additionally, the amount of GRFT released in vivo is dependent upon the amount of fiber intravaginally delivered. In line with our own observations and other's work, future studies evaluating NPs and NP-EF composites will assess if these platforms will provide extended protection against multiple challenges of in vivo HSV-2 infection.

In addition to providing GRFT release, the TPPs established the requirement that our GRFT delivery platforms must demonstrate acceptable safety profiles. Our in vivo and in vitro work have demonstrated the preliminary safety profiles of all delivery platforms. MTT analysis demonstrated that all platforms provided over $95 \%$ viability of vaginal cell lines. Additionally, no histological abnormalities were noted in murine tissues incubated with blank platforms. Although individual cytokines expression levels in some samples showed over a 2 to 3-fold increase relative to untreated controls, this was not observed with multiple cytokines, indicating negligible inflammatory response. However, further studies will be required to assess the safety of platforms, such as evaluating peripheral blood mononuclear cells (PMBCs) following intravaginal administration. 
Despite the success of these platforms, more work is required to assess the safety and efficacy of these platforms. First, in vivo murine models could be used to assess GRFT release, retention, and distribution within the reproductive tract as a function of administration time. Previous studies evaluating FDI and gel murine intravaginal retention found that GRFT released from these platforms was retained for longer durations in vivo than in vitro release studies suggested ${ }^{65,73}$. This disparity between release profiles has been attributed to the decreased wettability of the reproductive tract, resulting in delayed release of agents from platforms and increased retention of GRFT. Hence, the increased GRFT retention may translate to these platforms providing increased durations of protection against viral infections, than estimated from in vitro release studies. To validate this hypothesis, future studies may assess GRFT concentration in both reproductive tissue and vaginal lavages at selected timepoints following GRFT platform administration. To accomplish this, platforms incorporating fluorescently-labeled GRFT could be utilized, with murine reproductive tracts imaged for quantification of localized fluorescence at selected timepoints. We hypothesize that our GRFT platforms, in particular the GRFT NPs and NP-EF composites, will demonstrate prolonged retention and release, relative to the results observed during in vitro release studies.

Concurrent with assessing the retention and distribution of GRFT from delivery platforms is to evaluate the window of protection imparted by GRFT delivery vehicles. Our initial in vivo studies evaluated the efficacy of rapid-release and sustained-release delivery platforms $4 \mathrm{hr}$ and $24 \mathrm{hr}$ after administration, 
respectively. Immediate future work can assess if GRFT platforms protect against extended HSV-2 challenges, such as 1 wk, 1 month, or longer durations following platform administration. Additionally, multiple challenges of HSV-2 may be administered to assess if platforms provide protection in circumstances of multiple exposures to virus.

In addition to expanding the evaluation of these platforms against HSV-2 infection, other efficacy studies may assess the impact that multiple active agents with different mechanisms of action incorporated in fibers or NP-EF composites, may have on the dose needed to prevent HSV-2 infection. Although GRFT shows potent activity against HIV-1, it is less efficacious against HSV-2, demonstrating a 1000-fold higher $\mathrm{IC}_{50}$ for HSV-2, relative to HIV-1. Future formulations may incorporate other active agents tailored against HSV-2 (and HIV-1) infections, in addition to GRFT, to improve the dual-purpose utility of these platforms. We hypothesize that by incorporating additional active agents, future GRFT platforms will demonstrate synergistic efficacy against both HIV-1 and HSV-2 infections.

During our in vivo HSV-2 efficacy studies, it was found that blank EFs and NPs alone imparted partial protection against infection, relative to untreated/infected controls. Previous studies in our group have shown that the fiber structure itself can act as a barrier to viral particles, and inhibit cell entry. Future in vivo experiments may evaluate this property in order to enhance antiviral protection of delivery platforms. A potential experimental setup could determine how different fiber or composite structures (without active agent) could inhibit or prevent the spread of seminal fluid in murine models. Using seminal fluid 
containing fluorescent compounds mimicking viral particles or fluorescentlylabeled sperm, different fiber architectures could be assessed in mice to minimize virus transport throughout the reproductive tract. We hypothesize that fiber structures, in addition to providing release of incorporated agents, can be fabricated to as a physical barrier to virus distribution in tissue, imparting further protection.

Although we anticipate success of these platforms in providing sustainedrelease protection against viral infections, additional preclinical studies are required to fully ascertain the potential safety risks associated with administration of intravaginal delivery platforms, such as evaluating off-target effects and correlating with GRFT concentration and distribution in the reproductive tract. Previous safety studies with GRFT have demonstrated an outstanding safety profile, revealing that GRFT does not induce inflammation nor toxicity following subcutaneous or intravaginal administration. Although, intravaginal administration of GRFT gel in murine models results in GRFT distribution throughout the reproductive tract, including the uterine horns, it is considered unlikely for GRFT to absorb systemically. Similar results were observed in tests assessing polymers that comprising both EFs and NPs ${ }^{215}$, however, further testing will be required to validate the safety of our platforms. We hypothesize that our delivery platforms will continue to exhibit acceptable safety profiles in future studies that examine the pharmacodynamics and pharmacokinetics of released GRFT.

Lastly, we anticipate the need for additional studies to confirm the safety of these delivery platforms. Previous studies examining microbicide candidates, such 
as $\mathrm{N}-9$, showed that traditional safety studies failed to reveal the gel's cytotoxic properties, which enhanced the risk of viral acquisition, and exposed the need for further studies. A virus susceptibility study, run with the proper positive controls can be investigated to validate the complementary histological and cytokine studies conducted to date. Compounds known to increase viral susceptibility, such as $\mathrm{N}-9$ and benzalkonium chloride, will be administered as positive controls of toxicity and inflammation. We hypothesize that GRFT delivery platforms will not enhance the risk of HSV-2 acquisition, but that the positive controls will enhance susceptibility.

Based on our criteria and future studies, multilayered composites demonstrated the most promising potential for long-term GRFT release of the platforms examined. The composites provided GRFT release for up to $90 \mathrm{~d}$, and were designed to overcome the challenge of high mucus clearance within the FRT. However, it was noted that only $50 \%$ of incorporated GRFT released from NP-EF composites. Future studies will be necessary to account for the lack of complete release during the $90 \mathrm{~d}$ period. It is possible that residual GRFT may be noncovalently bound to the composite scaffold structure. Established extraction methods may be used to separate GRFT protein from polymeric scaffolds. Another possibility for low GRFT recovery is that GRFT was released completely, but was unable to be quantitated due to denaturation or alteration. Quantification of eluate samples from composite release studies can be analyzed using liquid chromatography-mass spectrometry to account for possible denatured or covalently modified GRFT compounds. Although GRFT has been characterized as 
highly stable and the high loading of rapid-release GRFT fibers results indicate that the electrospinning process does not affect the activity or structure of GRFT, further analysis will be required to validate the stability of incorporated GRFT from NP-EF composites.

Finally, in future work, the composite architectures may be more finely tailored to modulate active agent release. Altering polymer choice, electrospinning conditions, or the number of fiber layers, all have the potential to tune GRFT release from multilayered composites. Additionally, the shape and size of composites may be revisited to better serve user preference and needs. Concurrent with experimental composite alterations, mathematical modeling may be employed to better predict the release kinetics of agents from fiber delivery platforms. Previous work has shown the capacity of in silico modeling in predicting TDF release from PLGA fibers. By accounting for parameters involved in agent release, in silico models can complement and expedite the optimization of agent(s) release. This optimization may be helpful for composites, in which the fabrication of these complex structures is dependent upon dozens of interdependent parameters. 


\section{REFERENCES}

1 WHO. Sexually Transmitted Infections (STI) Fact Sheet <https://www.who.int/en/news-room/fact-sheets/detail/sexually-transmittedinfections-(stis) $>$ (2019).

2 Freeman, E. E. et al. Herpes simplex virus 2 infection increases HIV acquisition in men and women: systematic review and meta-analysis of longitudinal studies. AIDS 20, 73-83 (2006).

3 Corey, L., Wald, A., Celum, C. L. \& Quinn, T. C. The effects of herpes simplex virus-2 on HIV-1 acquisition and transmission: a review of two overlapping epidemics. J Acquir Immune Defic Syndr 35, 435-445 (2004).

4 Beyrer, C. et al. Global epidemiology of HIV infection in men who have sex with men. Lancet 380, 367-377, doi:10.1016/S0140-6736(12)60821-6 (2012).

5 Beyrer, C. \& Abdool Karim, Q. The changing epidemiology of HIV in 2013. Curr Opin HIV AIDS 8, 306-310, doi:10.1097/COH.0b013e328361f53a (2013).

6 Sharp, P. M. \& Hahn, B. H. Origins of HIV and the AIDS pandemic. Cold Spring Harb Perspect Med 1, a006841, doi:10.1101/cshperspect.a006841 (2011).

7 Aidsmap, N. Route and susceptibility: mucous membranes and target cells, $<\mathrm{http}: / /$ www.aidsmap.com/Route-and-susceptibility-mucous-membranes-andtarget-cells/page/1324028/> (2009).

8 Roser, M. R., H. HIV / AIDS, <https://ourworldindata.org/hiv-aids> (2019).

9 Braun, J. et al. A new quantitative HIV load assay based on plasma virion reverse transcriptase activity for the different types, groups and subtypes. AIDS 17, 331336, doi:10.1097/00002030-200302140-00006 (2003).

10 Loemba, $\mathrm{H}$. et al. Genetic divergence of human immunodeficiency virus type 1 Ethiopian clade $\mathrm{C}$ reverse transcriptase (RT) and rapid development of resistance against nonnucleoside inhibitors of RT. Antimicrob Agents Chemother 46, 20872094, doi:10.1128/aac.46.7.2087-2094.2002 (2002).

11 Taylor, B. S., Sobieszczyk, M. E., McCutchan, F. E. \& Hammer, S. M. The challenge of HIV-1 subtype diversity. $N$ Engl $J$ Med 358, 1590-1602, doi:10.1056/NEJMra0706737 (2008).

12 Sarafianos, S. G. et al. Structure and function of HIV-1 reverse transcriptase: molecular mechanisms of polymerization and inhibition. J Mol Biol 385, 693-713, doi:10.1016/j.jmb.2008.10.071 (2009).

13 Wilen, C. B., Tilton, J. C. \& Doms, R. W. Molecular mechanisms of HIV entry. Adv Exp Med Biol 726, 223-242, doi:10.1007/978-1-4614-0980-9_10 (2012).

14 Briz, V., Poveda, E. \& Soriano, V. HIV entry inhibitors: mechanisms of action and resistance pathways. J Antimicrob Chemother 57, 619-627, doi:10.1093/jac/dkl027 (2006).

15 De Clercq, E. Anti-HIV drugs: 25 compounds approved within 25 years after the discovery of HIV. International journal of antimicrobial agents 33, 307-320 (2009). 
16 Broder, S. The development of antiretroviral therapy and its impact on the HIV1/AIDS pandemic. Antiviral Res 85, 1-18, doi:10.1016/j.antiviral.2009.10.002 (2010).

17 Haqqani, A. A. \& Tilton, J. C. Entry inhibitors and their use in the treatment of HIV1 infection. Antiviral Research 98, 158-170, doi:https://doi.org/10.1016/j.antiviral.2013.03.017 (2013).

18 Wertheim, J. O., Smith, M. D., Smith, D. M., Scheffler, K. \& Kosakovsky Pond, S. L. Evolutionary origins of human herpes simplex viruses 1 and 2. Mol Biol Evol31, 2356-2364, doi:10.1093/molbev/msu185 (2014).

19 Johnston, C., Koelle, D. M. \& Wald, A. HSV-2: in pursuit of a vaccine. J Clin Invest 121, 4600-4609, doi:10.1172/JCI57148 (2011).

20 Brugha, R., Keersmaekers, K., Renton, A. \& Meheus, A. Genital herpes infection: a review. Int J Epidemiol 26, 698-709, doi:10.1093/ije/26.4.698 (1997).

21 Whitley, R. J. \& Roizman, B. Herpes simplex virus infections. Lancet 357, 15131518, doi:10.1016/S0140-6736(00)04638-9 (2001).

22 Rauch, D. A., Rodriguez, N. \& Roller, R. J. Mutations in herpes simplex virus glycoprotein D distinguish entry of free virus from cell-cell spread. $J$ Virol 74, 11437-11446, doi:10.1128/jvi.74.24.11437-11446.2000 (2000).

23 Campadelli-Fiume, G. et al. The multipartite system that mediates entry of herpes simplex virus into the cell. Reviews in Medical Virology 17, 313-326, doi:10.1002/rmv.546 (2007).

24 De Clercq, E. Antiviral drugs in current clinical use. Journal of Clinical Virology 30, 115-133, doi:https://doi.org/10.1016/j.jcv.2004.02.009 (2004).

Hodge, R. A. V. Famciclovir and Penciclovir. The Mode of Action of Famciclovir Including Its Conversion to Penciclovir. Antiviral Chemistry and Chemotherapy 4, 67-84, doi:10.1177/095632029300400201 (1993).

26 Fleming, D. T. et al. Herpes simplex virus type 2 in the United States, 1976 to 1994. N Engl J Med 337, 1105-1111, doi:10.1056/NEJM199710163371601 (1997).

27 Release, F. N. FDA approves first drug for reducing the risk of sexually acquired HIV infection., <https://aidsinfo.nih.gov/news/1254/fda-approves-first-drug-forreducing-the-risk-of-sexually-acquired-hiv-infection> (July 16, 2012 ).

28 Mastro, T. D., Sista, N. \& Abdool-Karim, Q. ARV-based HIV prevention for women - where we are in 2014. J Int AIDS Soc 17, 19154, doi:10.7448/IAS.17.3.19154 (2014).

29 Marrazzo, J. M. et al. Tenofovir-based preexposure prophylaxis for HIV infection among African women. N Engl J Med 372, 509-518, doi:10.1056/NEJMoa1402269 (2015).

30 Wira, C. R., Patel, M. V., Ghosh, M., Mukura, L. \& Fahey, J. V. Innate immunity in the human female reproductive tract: endocrine regulation of endogenous antimicrobial protection against HIV and other sexually transmitted infections. Am J Reprod Immunol 65, 196-211, doi:10.1111/j.1600-0897.2011.00970.x (2011).

31 Unnithan, A. R. et al. Wound-dressing materials with antibacterial activity from electrospun polyurethane-dextran nanofiber mats containing ciprofloxacin $\mathrm{HCl}$. Carbohydr Polym 90, 1786-1793, doi:10.1016/j.carbpol.2012.07.071 (2012).

32 Tourgeman, D. E., Gentzchein, E., Stanczyk, F. Z. \& Paulson, R. J. Serum and tissue hormone levels of vaginally and orally administered estradiol. Am J Obstet Gynecol 180, 1480-1483, doi:Doi 10.1016/S0002-9378(99)70042-6 (1999).

33 Steinbach, J. M. Protein and oligonucleotide delivery systems for vaginal microbicides against viral STIs. Cell Mol Life Sci 72, 469-503, doi:10.1007/s00018014-1756-3 (2015). 
34 Bernkop-Schnürch, A. \& Hornof, M. Intravaginal drug delivery systems. American Journal of Drug Delivery 1, 241-254, doi:10.2165/00137696-200301040-00003 (2003).

35 Boonstra, H. D. Multipurpose Prevention Technologies for the Developing World: U.S. Investment Is Critical. Guttmacher Policy Review 18, $62-69$ (2015).

36 Abdool Karim, Q. et al. Effectiveness and safety of tenofovir gel, an antiretroviral microbicide, for the prevention of HIV infection in women. Science 329, 1168-1174, doi:10.1126/science.1193748 (2010).

37 Shattock, R. J. \& Rosenberg, Z. Microbicides: topical prevention against HIV. Cold Spring Harbor perspectives in medicine 2, a007385-a007385, doi:10.1101/cshperspect.a007385 (2012).

38 Chou, S. F., Carson, D. \& Woodrow, K. A. Current strategies for sustaining drug release from electrospun nanofibers. J Control Release 220, 584-591, doi:10.1016/j.jconrel.2015.09.008 (2015).

39 Hickey, D. K., Patel, M. V., Fahey, J. V. \& Wira, C. R. Innate and adaptive immunity at mucosal surfaces of the female reproductive tract: stratification and integration of immune protection against the transmission of sexually transmitted infections. $J$ Reprod Immunol 88, 185-194, doi:10.1016/j.jri.2011.01.005 (2011).

40 Wiggins, R., Hicks, S. J., Soothill, P. W., Millar, M. R. \& Corfield, A. P. Mucinases and sialidases: their role in the pathogenesis of sexually transmitted infections in the female genital tract. Sex Transm Infect 77, 402-408, doi:10.1136/sti.77.6.402 (2001).

41 das Neves, J. \& Bahia, M. F. Gels as vaginal drug delivery systems. Int J Pharm 318, 1-14, doi:10.1016/j.jpharm.2006.03.012 (2006).

42 Andrews, G. P. et al. Characterization of the Rheological, Mucoadhesive, and Drug Release Properties of Highly Structured Gel Platforms for Intravaginal Drug Delivery. Biomacromolecules 10, 2427-2435, doi:10.1021/bm9003332 (2009).

43 Devlin, B., Nuttall, J., Wilder, S., Woodsong, C. \& Rosenberg, Z. Development of dapivirine vaginal ring for HIV prevention. Antiviral Res 100 Suppl, S3-8, doi:10.1016/j.antiviral.2013.09.025 (2013).

44 Derby, N., Zydowsky, T. \& Robbiani, M. In search of the optimal delivery method for anti-HIV microbicides: are intravaginal rings the way forward? Expert Rev AntiInfe 11, 5-8, doi:10.1586/Eri.12.155 (2013).

45 Ho, E., A. Intravaginal Rings as a Novel Platform for Mucosal Vaccination. Journal of Molecular Pharmaceutics \& Organic Process Research 1, 1-2 (2013).

46 Mallipeddi, R. \& Rohan, L. C. Nanoparticle-based vaginal drug delivery systems for HIV prevention. Expert Opin Drug Deliv 7, 37-48, doi:10.1517/17425240903338055 (2010).

47 Kiser, P. F., Johnson, T. J. \& Clark, J. T. State of the art in intravaginal ring technology for topical prophylaxis of HIV infection. AIDS Rev 14, 62-77 (2012).

48 Dieben, T. O. M., Roumen, F. J. M. E. \& Apter, D. Efficacy, cycle control, and user acceptability of a novel combined contraceptive vaginal ring. Obstet Gynecol 100, 585-593, doi:Pii S0029-7844(02)02124-5Doi 10.1016/S0029-7844(02)02124-5 (2002).

49 Malcolm, R. K., Edwards, K. L., Kiser, P., Romano, J. \& Smith, T. J. Advances in microbicide vaginal rings. Antiviral Res 88 Suppl 1, S30-39, doi:10.1016/j.antiviral.2010.09.003 (2010).

50 Roumen, F. J. M. E., Apter, D., Mulders, T. M. T. \& Deiben, T. O. M. Efficacy, tolerability and acceptability of a novel contraceptive vaginal ring releasing etonogestrel and ethinyl oestradiol. Hum Reprod 16, 469-475, doi:DOI 10.1093/humrep/16.3.469 (2001). 

in Women. N Engl J Med 375, 2133-2143, doi:10.1056/NEJMoa1602046 (2016).

52 Kim, S., Traore, Y. L., Chen, Y., Ho, E. A. \& Liu, S. Switchable On-Demand Release of a Nanocarrier from a Segmented Reservoir Type Intravaginal Ring Filled with a pH-Responsive Supramolecular Polyurethane Hydrogel. ACS Applied Bio Materials 1, 652-662, doi:10.1021/acsabm.8b00146 (2018).

53 Hankins, C. A. \& Dybul, M. R. The promise of pre-exposure prophylaxis with antiretroviral drugs to prevent HIV transmission: a review. Current Opinion in HIV and AIDS 8, 50-58, doi:10.1097/COH.0b013e32835b809d (2013).

54 Akil, A. et al. Formulation and characterization of polymeric films containing combinations of antiretrovirals (ARVs) for HIV prevention. Pharm Res 32, 458-468, doi:10.1007/s11095-014-1474-4 (2015).

55 Baeten, J. M. et al. Use of a Vaginal Ring Containing Dapivirine for HIV-1 Prevention in Women. The New England journal of medicine 375, 2121-2132, doi:10.1056/NEJMoa1506110 (2016).

56 Mesquita, P. M. et al. Intravaginal ring delivery of tenofovir disoproxil fumarate for prevention of HIV and herpes simplex virus infection. J Antimicrob Chemother 67, 1730-1738, doi:10.1093/jac/dks097 (2012).

57 Smith, J. M. et al. Intravaginal ring eluting tenofovir disoproxil fumarate completely protects macaques from multiple vaginal simian-HIV challenges. Proc Natl Acad Sci U S A 110, 16145-16150, doi:10.1073/pnas.1311355110 (2013).

Moss, J. A. et al. Pharmacokinetics and preliminary safety study of podintravaginal rings delivering antiretroviral combinations for HIV prophylaxis in a macaque model. Antimicrobial agents and chemotherapy 58, 5125-5135, doi:10.1128/AAC.02871-14 (2014).

$59 \mathrm{NIH}$. Most women use vaginal ring for HIV prevention in open-label study, $<$ https://www.nih.gov/news-events/news-releases/most-women-use-vaginal-ringhiv-prevention-open-label-study> (2019).

60 Revers, L. \& Furczon, E. An Introduction to Biologics and Biosimilars. Part I: Biologics: What are They and Where Do They Come from? Canadian Pharmacists Journal / Revue des Pharmaciens du Canada 143, 134-139, doi:10.3821/1913701X-143.3.134 (2010).

61 Mori, T. et al. Isolation and characterization of griffithsin, a novel HIV-inactivating protein, from the red alga Griffithsia sp. J Biol Chem 280, 9345-9353, doi:10.1074/jbc.M411122200 (2005).

62 O'Keefe, B. R. et al. Broad-spectrum in vitro activity and in vivo efficacy of the antiviral protein griffithsin against emerging viruses of the family Coronaviridae. $J$ Virol 84, 2511-2521, doi:10.1128/JVI.02322-09 (2010).

63 Meuleman, P. et al. Griffithsin has antiviral activity against hepatitis $C$ virus. Antimicrob Agents Chemother 55, 5159-5167, doi:10.1128/AAC.00633-11 (2011).

64 Ishag, H. Z. et al. Griffithsin inhibits Japanese encephalitis virus infection in vitro and in vivo. Arch Virol 158, 349-358, doi:10.1007/s00705-012-1489-2 (2013).

65 Nixon, B. et al. Griffithsin protects mice from genital herpes by preventing cell-tocell spread. J Virol 87, 6257-6269, doi:10.1128/JVI.00012-13 (2013).

66 Emau, P. et al. Griffithsin, a potent HIV entry inhibitor, is an excellent candidate for anti-HIV microbicide. J Med Primatol 36, 244-253, doi:10.1111/j.16000684.2007.00242.x (2007).

67 Xue, J. et al. The griffithsin dimer is required for high-potency inhibition of HIV-1: evidence for manipulation of the structure of gp120 as part of the griffithsin dimer mechanism. Antimicrob Agents Chemother 57, 3976-3989, doi:10.1128/AAC.00332-13 (2013). 
68 Kouokam, J. C., Lasnik, A. B. \& Palmer, K. E. Studies in a Murine Model Confirm the Safety of Griffithsin and Advocate Its Further Development as a Microbicide Targeting HIV-1 and Other Enveloped Viruses. Viruses 8, doi:10.3390/v8110311 (2016).

69 Kouokam, J. C. et al. Investigation of griffithsin's interactions with human cells confirms its outstanding safety and efficacy profile as a microbicide candidate. PLoS One 6, e22635, doi:10.1371/journal.pone.0022635 (2011).

70 Ferir, G. et al. Combinations of griffithsin with other carbohydrate-binding agents demonstrate superior activity against HIV Type 1, HIV Type 2, and selected carbohydrate-binding agent-resistant HIV Type 1 strains. AIDS Res Hum Retroviruses 28, 1513-1523, doi:10.1089/AID.2012.0026 (2012).

71 Levendosky, K. et al. Griffithsin and Carrageenan Combination To Target Herpes Simplex Virus 2 and Human Papillomavirus. Antimicrob Agents Chemother 59, 7290-7298, doi:10.1128/AAC.01816-15 (2015).

72 Ferir, G., Palmer, K. E. \& Schols, D. Synergistic activity profile of griffithsin in combination with tenofovir, maraviroc and enfuvirtide against HIV-1 clade $\mathrm{C}$. Virology 417, 253-258, doi:10.1016/j.virol.2011.07.004 (2011).

73 Derby, N. et al. Griffithsin carrageenan fast dissolving inserts prevent SHIV HSV2 and HPV infections in vivo. Nat Commun 9, doi:ARTN 388110.1038/s41467-01806349-0 (2018).

74 Lal, M. et al. Development of a Vaginal Fast-Dissolving Insert Combining Griffithsin and Carrageenan for Potential Use Against Sexually Transmitted Infections. J Pharm Sci-Us 107, 2601-2610, doi:10.1016/j.xphs.2018.06.002 (2018).

75 Grooms, T. N. et al. Griffithsin-Modified Electrospun Fibers as a Delivery Scaffold To Prevent HIV Infection. Antimicrob Agents Chemother 60, 6518-6531, doi:10.1128/AAC.00956-16 (2016).

76 Pillay, V. et al. A Review of the Effect of Processing Variables on the Fabrication of Electrospun Nanofibers for Drug Delivery Applications. J Nanomater, doi:Artn 78928910.1155/2013/789289 (2013).

77 Gunatillake, P. A. \& Adhikari, R. Biodegradable synthetic polymers for tissue engineering. Eur Cell Mater 5, 1-16; discussion 16 (2003).

78 Makadia, H. K. \& Siegel, S. J. Poly Lactic-co-Glycolic Acid (PLGA) as Biodegradable Controlled Drug Delivery Carrier. Polymers (Basel) 3, 1377-1397, doi:10.3390/polym3031377 (2011).

79 Zamani, M., Prabhakaran, M. P. \& Ramakrishna, S. Advances in drug delivery via electrospun and electrosprayed nanomaterials. Int J Nanomedicine 8, 2997-3017, doi:10.2147/IJN.S43575 (2013).

$80 \mathrm{Hu}, \mathrm{X}$. L. et al. Electrospinning of polymeric nanofibers for drug delivery applications. Journal of Controlled Release 185, 12-21, doi:10.1016/j.jconrel.2014.04.018 (2014).

81 Jain, K. K. Drug delivery systems. Vol. 2 (Springer, 2008).

82 Sharma, R. et al. Recent advances in polymeric electrospun nanofibers for drug delivery. Crit Rev Ther Drug Carrier Syst 31, 187-217 (2014).

83 Huang, C. et al. Electrospun cellulose acetate phthalate fibers for semen induced anti-HIV vaginal drug delivery. Biomaterials 33, 962-969, doi:10.1016/j.biomaterials.2011.10.004 (2012).

84 Huang, C. et al. Electrospun polystyrene fibers for HIV entrapment. Polymers for Advanced Technologies 25, 827-834, doi:10.1002/pat.3310 (2014).

85 Carson, D., Jiang, Y. \& Woodrow, K. A. Tunable Release of Multiclass Anti-HIV Drugs that are Water-Soluble and Loaded at High Drug Content in Polyester 
Blended Electrospun Fibers. Pharm Res 33, 125-136, doi:10.1007/s11095-0151769-0 (2016).

86 Ball, C., Krogstad, E., Chaowanachan, T. \& Woodrow, K. A. Drug-eluting fibers for HIV-1 inhibition and contraception. PLoS One 7, e49792, doi:10.1371/journal.pone.0049792 (2012).

87 Aniagyei, S. E. et al. Evaluation of poly(lactic-co-glycolic acid) and poly(dl-lactideco- $\varepsilon$-caprolactone) electrospun fibers for the treatment of HSV-2 infection. Materials Science and Engineering: C 72, 238-251, doi:https://doi.org/10.1016/j.msec.2016.11.029 (2017).

88 Repanas, A., Andriopoulou, S. \& Glasmacher, B. The significance of electrospinning as a method to create fibrous scaffolds for biomedical engineering and drug delivery applications. J Drug Deliv Sci Tec 31, 137-146, doi:10.1016/j.jddst.2015.12.007 (2016).

89 Fu, Y. \& Kao, W. J. Drug release kinetics and transport mechanisms of nondegradable and degradable polymeric delivery systems. Expert Opin Drug Deliv 7, 429-444, doi:10.1517/17425241003602259 (2010).

90 Kim, T. G., Lee, D. S. \& Park, T. G. Controlled protein release from electrospun biodegradable fiber mesh composed of poly(epsilon-caprolactone) and poly(ethylene oxide). Int J Pharm 338, 276-283, doi:10.1016/j.ijpharm.2007.01.040 (2007).

91 Qi, H., Hu, P., Xu, J. \& Wang, A. Encapsulation of drug reservoirs in fibers by emulsion electrospinning: morphology characterization and preliminary release assessment. Biomacromolecules 7, 2327-2330, doi:10.1021/bm060264z (2006).

92 Nair, L. S. \& Laurencin, C. T. Biodegradable polymers as biomaterials. Prog Polym Sci 32, 762-798, doi:10.1016/j.progpolymsci.2007.05.017 (2007).

93 Liu, H., Leonas, K. K. \& Zhao, Y. P. Antimicrobial Properties and Release Profile of Ampicillin from Electrospun Poly(epsilon-caprolactone) Nanofiber Yarns. J Eng Fiber Fabr 5, 10-19 (2010).

94 Yoshimoto, H., Shin, Y. M., Terai, H. \& Vacanti, J. P. A biodegradable nanofiber scaffold by electrospinning and its potential for bone tissue engineering. Biomaterials 24, 2077-2082 (2003).

95 Luu, Y. K., Kim, K., Hsiao, B. S., Chu, B. \& Hadjiargyrou, M. Development of a nanostructured DNA delivery scaffold via electrospinning of PLGA and PLA-PEG block copolymers. J Control Release 89, 341-353 (2003).

96 Puppi, D. et al. Nano/microfibrous polymeric constructs loaded with bioactive agents and designed for tissue engineering applications: a review. J Biomed Mater Res B Appl Biomater 102, 1562-1579, doi:10.1002/jbm.b.33144 (2014).

97 Cipitria, A., Skelton, A., Dargaville, T. R., Dalton, P. D. \& Hutmacher, D. W. Design, fabrication and characterization of PCL electrospun scaffolds-a review. J Mater Chem 21, 9419-9453, doi:10.1039/c0jm04502k (2011).

98 Uhrich, K. E., Cannizzaro, S. M., Langer, R. S. \& Shakesheff, K. M. Polymeric systems for controlled drug release. Chemical reviews 99, 3181-3198 (1999).

99 Ulery, B. D., Nair, L. S. \& Laurencin, C. T. Biomedical Applications of Biodegradable Polymers. J Polym Sci B Polym Phys 49, 832-864, doi:10.1002/polb.22259 (2011).

100 Chen, D. W. \& Liu, S. J. Nanofibers used for delivery of antimicrobial agents. Nanomedicine (Lond) 10, 1959-1971, doi:10.2217/nnm.15.28 (2015).

101 Blakney, A. K., Ball, C., Krogstad, E. A. \& Woodrow, K. A. Electrospun fibers for vaginal anti-HIV drug delivery. Antiviral Res 100 Suppl, S9-16, doi:10.1016/j.antiviral.2013.09.022 (2013). 
102 Ramakrishna, S. et al. Electrospun nanofibers: solving global issues. Mater Today 9, 40-50, doi:Doi 10.1016/S1369-7021(06)71389-X (2006).

103 Gopferich, A. Mechanisms of polymer degradation and erosion. Biomaterials 17, 103-114, doi:Doi 10.1016/0142-9612(96)85755-3 (1996).

104 Chou, S. F. \& Woodrow, K. A. Relationships between mechanical properties and drug release from electrospun fibers of PCL and PLGA blends. J Mech Behav Biomed Mater 65, 724-733, doi:10.1016/j.jmbbm.2016.09.004 (2017).

105 von Burkersroda, F., Schedl, L. \& Gopferich, A. Why degradable polymers undergo surface erosion or bulk erosion. Biomaterials 23, 4221-4231 (2002).

106 Doshi, J. \& Reneker, D. H. Electrospinning Process and Applications of Electrospun Fibers. J Electrostat 35, 151-160, doi:Doi 10.1016/03043886(95)00041-8 (1995).

107 Zeng, J. et al. Biodegradable electrospun fibers for drug delivery. Journal of controlled release 92, 227-231 (2003).

108 Sill, T. J. \& von Recum, H. A. Electrospinning: applications in drug delivery and tissue engineering. Biomaterials 29, 1989-2006, doi:10.1016/j.biomaterials.2008.01.011 (2008).

109 Kenawy, E., Abdel-Hay, F. I., El-Newehy, M. H. \& Wnek, G. E. Processing of Polymer Nanofibers through Electrospinning as Drug Delivery Systems. Nato Sci Peace Secur, 247-+, doi:Doi 10.1007/978-1-4020-9491-0_19 (2009).

$110 \mathrm{Ji}, \mathrm{W}$. et al. Bioactive electrospun scaffolds delivering growth factors and genes for tissue engineering applications. Pharm Res 28, 1259-1272, doi:10.1007/s11095010-0320-6 (2011).

111 Xie, J., Li, X. \& Xia, Y. Putting Electrospun Nanofibers to Work for Biomedical Research. Macromol Rapid Commun 29, 1775-1792, doi:10.1002/marc.200800381 (2008).

112 Subbiah, T., Bhat, G., Tock, R., Parameswaran, S. \& Ramkumar, S. Electrospinning of nanofibers. Journal of applied polymer science 96, 557-569 (2005).

113 Hadjiargyrou, M. \& Chiu, J. B. Enhanced composite electrospun nanofiber scaffolds for use in drug delivery. Expert Opin Drug Deliv 5, 1093-1106, doi:10.1517/17425247.5.10.1093 (2008).

114 Zhang, Y. Z., Lim, C. T., Ramakrishna, S. \& Huang, Z. M. Recent development of polymer nanofibers for biomedical and biotechnological applications. J Mater SciMater M 16, 933-946, doi:10.1007/s10856-005-4428-x (2005).

115 Verreck, G. et al. Incorporation of drugs in an amorphous state into electrospun nanofibers composed of a water-insoluble, nonbiodegradable polymer. J Control Release 92, 349-360 (2003).

116 Han, D. \& Steckl, A. J. Triaxial electrospun nanofiber membranes for controlled dual release of functional molecules. ACS Appl Mater Interfaces 5, 8241-8245, doi:10.1021/am402376c (2013).

117 Yarin, A. L. Coaxial electrospinning and emulsion electrospinning of core-shell fibers. Polymers for Advanced Technologies 22, 310-317, doi:10.1002/pat.1781 (2011).

118 He, C. L. et al. Coaxial electrospun poly(L-lactic acid) ultrafine fibers for sustained drug delivery. J Macromol Sci B 45, 515-524, doi:10.1080/00222340600769832 (2006).

119 Lu, Y. et al. Coaxial electrospun fibers: applications in drug delivery and tissue engineering. Wires Nanomed Nanobi 8, 654-677, doi:10.1002/wnan.1391 (2016). 
$120 \mathrm{Yu}, \mathrm{D}$. G. et al. Improving polymer nanofiber quality using a modified co-axial electrospinning process. Macromol Rapid Commun 32, 744-750, doi:10.1002/marc.201100049 (2011).

121 Nezarati, R. M., Eifert, M. B. \& Cosgriff-Hernandez, E. Effects of humidity and solution viscosity on electrospun fiber morphology. Tissue Eng Part C Methods 19, 810-819, doi:10.1089/ten.TEC.2012.0671 (2013).

122 Wang, J. R., Jakli, A. \& West, J. L. Morphology Tuning of Electrospun Liquid Crystal/Polymer Fibers. Chemphyschem 17, 3080-3085, doi:10.1002/cphc.201600430 (2016).

123 Yang, J. M., Zha, L. S., Yu, D. G. \& Liu, J. Y. Coaxial electrospinning with acetic acid for preparing ferulic acid/zein composite fibers with improved drug release profiles. Colloid Surface B 102, 737-743, doi:10.1016/j.colsurfb.2012.09.039 (2013).

124 Tang, C., Ozcam, A. E., Stout, B. \& Khan, S. A. Effect of pH on Protein Distribution in Electrospun PVA/BSA Composite Nanofibers. Biomacromolecules 13, 12691278, doi:10.1021/bm2017146 (2012).

125 He, M., Jiang, H. Y., Wang, R., Xie, Y. \& Zhao, C. S. Fabrication of metrpnidazole loaded poly (epsilon-caprolactone)/zein core/shell nanofiber membranes via coaxial electrospinning for guided tissue regeneration. J Colloid Interf Sci 490, 270278, doi:10.1016/j.jcis.2016.11.062 (2017).

126 Wang, C., Yan, K. W., Lin, Y. D. \& Hsieh, P. C. H. Biodegradable Core/Shell Fibers by Coaxial Electrospinning: Processing, Fiber Characterization, and Its Application in Sustained Drug Release. Macromolecules 43, 6389-6397, doi:10.1021/ma100423x (2010).

127 Perrie, Y. \& Rades, T. FASTtrack Pharmaceutics: Drug Delivery and Targeting. (Pharmaceutical press, 2012).

128 Jiang, Y. N., Mo, H. Y. \& Yu, D. G. Electrospun drug-loaded core-sheath PVP/zein nanofibers for biphasic drug release. Int $J$ Pharm 438, 232-239, doi:10.1016/j.jpharm.2012.08.053 (2012).

129 Zhu, L. F., Liu, X. Y., Du, L. N. \& Jin, Y. G. Preparation of asiaticoside-loaded coaxially electrospinning nanofibers and their effect on deep partial-thickness burn injury. Biomed Pharmacother 83, 33-40, doi:10.1016/j.biopha.2016.06.016 (2016).

130 Castillo-Ortega, M. M. et al. Preparation by coaxial electrospinning and characterization of membranes releasing (-) epicatechin as scaffold for tissue engineering. Mat Sci Eng C-Mater 46, 184-189, doi:10.1016/j.msec.2014.10.031 (2015).

131 Li, X. Y., Li, Y. C., Yu, D. G., Liao, Y. Z. \& Wang, X. Fast Disintegrating QuercetinLoaded Drug Delivery Systems Fabricated Using Coaxial Electrospinning. Int $J$ Mol Sci 14, 21647-21659, doi:10.3390/ijms141121647 (2013).

$132 \mathrm{Yu}, \mathrm{D} . \mathrm{G}$. et al. Solid dispersions in the form of electrospun core-sheath nanofibers. Int J Nanomed 6, 3271-3280, doi:10.2147/ljn.S27468 (2011).

$133 \mathrm{Fu}$, L. N., Zhang, J. \& Yang, G. Present status and applications of bacterial cellulose-based materials for skin tissue repair. Carbohyd Polym 92, 1432-1442, doi:10.1016/j.carbpol.2012.10.071 (2013).

$134 \mathrm{Yu}, \mathrm{D}$. G. et al. Nanofibers Fabricated Using Triaxial Electrospinning as Zero Order Drug Delivery Systems. Acs Appl Mater Inter 7, 18891-18897, doi:10.1021/acsami.5b06007 (2015).

135 Nakielski, P. et al. Hydrogel Nanofilaments via Core-Shell Electrospinning (vol 10, e0129816, 2015). Plos One 10, doi:ARTN e013345810.1371/journal.pone.0133458 (2015). 
136 Zhu, Y. J. \& Chen, F. pH-Responsive Drug-Delivery Systems. Chem-Asian J 10, 284-305, doi:10.1002/asia.201402715 (2015).

137 Arnold, K. B. et al. Increased levels of inflammatory cytokines in the female reproductive tract are associated with altered expression of proteases, mucosal barrier proteins, and an influx of HIV-susceptible target cells. Mucosal Immunol 9, 194-205, doi:10.1038/mi.2015.51 (2016).

138 Thakral, S., Thakral, N. K. \& Majumdar, D. K. Eudragit (R): a technology evaluation. Expert Opin Drug Del 10, 131-149, doi:10.1517/17425247.2013.736962 (2013).

139 Yoshida, T., Lai, T. C., Kwon, G. S. \& Sako, K. pH- and ion-sensitive polymers for drug delivery. Expert Opin Drug Del 10, 1497-1513, doi:10.1517/17425247.2013.821978 (2013).

140 Jin, M., Yu, D. G., Geraldes, C. F. G. C., Williams, G. R. \& Bligh, S. W. A. Theranostic Fibers for Simultaneous Imaging and Drug Delivery. Mol Pharmaceut 13, 2457-2465, doi:10.1021/acs.molpharmaceut.6b00197 (2016).

141 Jia, D., Gao, Y. S. \& Williams, G. R. Core/shell poly(ethylene oxide)/Eudragit fibers for site-specific release. Int $J$ Pharm 523, 376-385, doi:10.1016/j.ijpharm.2017.03.038 (2017).

142 Hua, D. et al. pH responsive polyurethane (core) and cellulose acetate phthalate (shell) electrospun fibers for intravaginal drug delivery. Carbohydr Polym 151, 1240-1244, doi:10.1016/j.carbpol.2016.06.066 (2016).

143 Sang, Q. Q., Li, H. Y., Williams, G., Wu, H. L. \& Zhu, L. M. Core-shell poly(lactideco-epsilon-caprolactone)-gelatin fiber scaffolds as $\mathrm{pH}$-sensitive drug delivery systems. J Biomater App/ 32, 1105-1118, doi:10.1177/0885328217749962 (2018).

144 Han, D., Yu, X. J., Chai, Q. Y., Ayres, N. \& Steckl, A. J. Stimuli-Responsive SelfImmolative Polymer Nanofiber Membranes Formed by Coaxial Electrospinning. Acs Appl Mater Inter 9, 11858-11865, doi:10.1021/acsami.6b16501 (2017).

145 Ball, C., Chou, S. F., Jiang, Y. \& Woodrow, K. A. Coaxially electrospun fiber-based microbicides facilitate broadly tunable release of maraviroc. Mat Sci Eng C-Mater 63, 117-124, doi:10.1016/j.msec.2016.02.018 (2016).

146 Zhang, Y. Z. et al. Coaxial electrospinning of (fluorescein isothiocyanateconjugated bovine serum albumin)-encapsulated poly(epsilon-caprolactone) nanofibers for sustained release. Biomacromolecules 7, 1049-1057, doi:10.1021/bm050743i (2006).

$147 \mathrm{Yu}, \mathrm{H}$. et al. Regulation of biphasic drug release behavior by graphene oxide in polyvinyl pyrrolidone/poly(epsilon-caprolactone) core/sheath nanofiber mats. Colloid Surface B 146, 63-69, doi:10.1016/j.colsurfb.2016.05.052 (2016).

148 Oliveira, M. F. et al. Electrospun nanofibers of polyCD/PMAA polymers and their potential application as drug delivery system. Mat Sci Eng C-Mater 54, 252-261, doi:10.1016/j.msec.2015.04.042 (2015).

149 Sultanova, Z., Kaleli, G., Kabay, G. \& Mutlu, M. Controlled release of a hydrophilic drug from coaxially electrospun polycaprolactone nanofibers. Int $J$ Pharm 505, 133-138, doi:10.1016/j.jpharm.2016.03.032 (2016).

150 Lv, Y. et al. Core-Sheath Nanofibers as Drug Delivery System for Thermoresponsive Controlled Release. J Pharm Sci-Us 106, 1258-1265, doi:10.1016/j.xphs.2016.12.031 (2017).

151 Khalf, A. \& Madihally, S. V. Modeling the permeability of multiaxial electrospun poly(epsilon-caprolactone)-gelatin hybrid fibers for controlled doxycycline release. Mat Sci Eng C-Mater 76, 161-170, doi:10.1016/j.msec.2017.03.093 (2017).

152 Ranjbar-Mohammadi, M., Zamani, M., Prabhakaran, M. P., Bahrami, S. H. \& Ramakrishna, S. Electrospinning of PLGA/gum tragacanth nanofibers containing 
tetracycline hydrochloride for periodontal regeneration. Mat Sci Eng C-Mater 58, 521-531, doi:10.1016/j.msec.2015.08.066 (2016).

153 Xie, Q. et al. Fabrication of Core-Shell PEI/pBMP2-PLGA Electrospun Scaffold for Gene Delivery to Periodontal Ligament Stem Cells. Stem Cells Int, doi:Artn 538513710.1155/2016/5385137 (2016).

154 Jiang, H. L., Hu, Y. Q., Zhao, P. C., Li, Y. \& Zhu, K. J. Modulation of protein release from biodegradable core-shell structured fibers prepared by coaxial electrospinning. J Biomed Mater Res B 79b, 50-57, doi:10.1002/jbm.b.30510 (2006).

155 Jiang, H. L. et al. A facile technique to prepare biodegradable coaxial electrospun nanofibers for controlled release of bioactive agents. Journal of Controlled Release 108, 237-243, doi:10.1016/j.jconrel.2005.08.006 (2005).

156 Yang, Y., Li, X. H., Qi, M. B., Zhou, S. B. \& Weng, J. Release pattern and structural integrity of lysozyme encapsulated in core-sheath structured poly(DL-lactide) ultrafine fibers prepared by emulsion electrospinning. Eur J Pharm Biopharm 69, 106-116, doi:10.1016/j.ejpb.2007.10.016 (2008).

$157 \mathrm{Ji}, \mathrm{W}$. et al. Fibrous scaffolds loaded with protein prepared by blend or coaxial electrospinning. Acta Biomater 6, 4199-4207, doi:10.1016/j.actbio.2010.05.025 (2010).

158 Saraf, A., Baggett, L. S., Raphael, R. M., Kasper, F. K. \& Mikos, A. G. Regulated non-viral gene delivery from coaxial electrospun fiber mesh scaffolds. Journal of Controlled Release 143, 95-103, doi:10.1016/j.jconrel.2009.12.009 (2010).

$159 \mathrm{Hsu}, \mathrm{Y}$. H. et al. Dual delivery of active antibactericidal agents and bone morphogenetic protein at sustainable high concentrations using biodegradable sheath-core-structured drug-eluting nanofibers. Int J Nanomed 11, 3927-3937, doi:10.2147/ljn.S107250 (2016).

160 Ball, C. \& Woodrow, K. A. Electrospun solid dispersions of Maraviroc for rapid intravaginal preexposure prophylaxis of HIV. Antimicrob Agents Chemother 58, 4855-4865, doi:10.1128/AAC.02564-14 (2014).

161 Tyo, K. M. et al. Multipurpose tenofovir disoproxil fumarate electrospun fibers for the prevention of HIV-1 and HSV-2 infections in vitro. Int J Pharm 531, 118-133, doi:10.1016/j.jpharm.2017.08.061 (2017).

162 Tyo, K. M. et al. pH-responsive delivery of Griffithsin from electrospun fibers. Eur J Pharm Biopharm 138, 64-74 (2019).

163 Liu, L. et al. Nano-on-micro fibrous extracellular matrices for scalable expansion of human ES/iPS cells. Biomaterials 124, 47-54, doi:10.1016/j.biomaterials.2017.01.039 (2017).

164 Huang, L. Y., Branford-White, C., Shen, X. X., Yu, D. G. \& Zhu, L. M. Timeengineeringed biphasic drug release by electrospun nanofiber meshes. Int $J$ Pharm 436, 88-96, doi:10.1016/j.ijpharm.2012.06.058 (2012).

165 Meinel, A. J., Germershaus, O., Luhmann, T., Merkle, H. P. \& Meinel, L. Electrospun matrices for localized drug delivery: Current technologies and selected biomedical applications. Eur $J$ Pharm Biopharm 81, 1-13, doi:10.1016/j.ejpb.2012.01.016 (2012).

166 Blakney, A. K., Krogstad, E. A., Jiang, Y. H. \& Woodrow, K. A. Delivery of multipurpose prevention drug combinations from electrospun nanofibers using composite microarchitectures. Int J Nanomedicine 9, 2967-2978, doi:10.2147/IJN.S61664 (2014).

167 Mehrotra, S. et al. Time Controlled Protein Release from Layer-by-Layer Assembled Multilayer Functionalized Agarose Hydrogels. Adv Funct Mater 20, 247-258, doi:10.1002/adfm.200901172 (2010). 
168 Pan, H., Li, L. M., Hu, L. \& Cui, X. J. Continuous aligned polymer fibers produced by a modified electrospinning method. Polymer 47, 4901-4904, doi:10.1016/j.polymer.2006.05.012 (2006).

169 Shin, J. W. et al. Hybrid nanofiber scaffolds of polyurethane and poly(ethylene oxide) using dual-electrospinning for vascular tissue engineering. Ifmbe Proc 15, 692-+ (2007).

170 Baker, B. M. et al. The potential to improve cell infiltration in composite fiberaligned electrospun scaffolds by the selective removal of sacrificial fibers. Biomaterials 29, 2348-2358, doi:10.1016/j.biomaterials.2008.01.032 (2008).

171 Tijing, L. D. et al. One-step fabrication of antibacterial (silver nanoparticles/poly(ethylene oxide)) - Polyurethane bicomponent hybrid nanofibrous mat by dual-spinneret electrospinning. Mater Chem Phys 134, 557561, doi:10.1016/j.matchemphys.2012.03.037 (2012).

172 Wulkersdorfer, B. et al. Bimodal porous scaffolds by sequential electrospinning of poly (glycolic acid) with sucrose particles. International Journal of Polymer Science 2010 (2010).

173 Wan, A. C. A. \& Ying, J. Y. Nanomaterials for in situ cell delivery and tissue regeneration. Adv Drug Deliver Rev 62, 731-740, doi:10.1016/j.addr.2010.02.002 (2010).

174 Dvir, T., Timko, B. P., Kohane, D. S. \& Langer, R. Nanotechnological strategies for engineering complex tissues. Nat Nanotechnol 6, 13-22, doi:10.1038/Nnano.2010.246 (2011).

175 Kharaziha, M., Fathi, M. H. \& Edris, H. Tunable cellular interactions and physical properties of nanofibrous PCL-forsterite:gelatin scaffold through sequential electrospinning. Compos Sci Technol 87, 182-188, doi:10.1016/j.compscitech.2013.08.015 (2013).

176 Tan, L., Hu, J. L. \& Zhao, H. F. Design of bilayered nanofibrous mats for wound dressing using an electrospinning technique. Mater Lett 156, 46-49, doi:10.1016/j.matlet.2015.04.119 (2015).

177 Falde, E. J. et al. Layered superhydrophobic meshes for controlled drug release. Journal of Controlled Release 214, 23-29, doi:10.1016/j.jconrel.2015.06.042 (2015).

178 Sirc, J. et al. Controlled gentamicin release from multi-layered electrospun nanofibrous structures of various thicknesses. Int $J$ Nanomed 7, 5315-5325, doi:10.2147/ljn.S35781 (2012).

179 Mandal, B. B., Mann, J. K. \& Kundu, S. C. Silk fibroin/gelatin multilayered films as a model system for controlled drug release. Eur J Pharm Sci 37, 160-171, doi:10.1016/j.ejps.2009.02.005 (2009).

180 Okuda, T., Tominaga, K. \& Kidoaki, S. Time-programmed dual release formulation by multilayered drug-loaded nanofiber meshes. Journal of Controlled Release 143, 258-264, doi:10.1016/j.jconrel.2009.12.029 (2010).

181 Liu, S. et al. Use of asymmetric multilayer polylactide nanofiber mats in controlled release of drugs and prevention of liver cancer recurrence after surgery in mice. Nanomed-Nanotechnol 11, 1047-1056, doi:10.1016/j.nano.2015.03.001 (2015).

182 Chunder, A., Sarkar, S., Yu, Y. B. \& Zhai, L. Fabrication of ultrathin polyelectrolyte fibers and their controlled release properties. Colloid Surface B 58, 172-179, doi:10.1016/j.colsurfb.2007.03.004 (2007).

183 Son, Y. J., Kim, W. J. \& Yoo, H. S. Therapeutic applications of electrospun nanofibers for drug delivery systems. Arch Pharm Res 37, 69-78, doi:10.1007/s12272-013-0284-2 (2014). 
184 Yoon, H. \& Kim, G. H. Layer-by-layered electrospun micro/nanofibrous mats for drug delivery system. Macromol Res 20, 402-406, doi:10.1007/s13233-012-00479 (2012).

185 Park, J. H., Kim, B. S., Yoo, Y. C., Khil, M. S. \& Kim, H. Y. Enhanced mechanical properties of multilayer nano-coated electrospun nylon 6 fibers via a layer-by-layer self-assembly. Journal of Applied Polymer Science 107, 2211-2216, doi:10.1002/app.27322 (2008).

186 Ensign, L. M. et al. Mucus-penetrating nanoparticles for vaginal drug delivery protect against herpes simplex virus. Sci Transl Med 4, 138ra179, doi:10.1126/scitransImed.3003453 (2012).

187 Woodrow, K. A. et al. Intravaginal gene silencing using biodegradable polymer nanoparticles densely loaded with small-interfering RNA. Nat Mater 8, 526-533, doi:10.1038/nmat2444 (2009).

188 Ensign, L. M., Cone, R. \& Hanes, J. Nanoparticle-based drug delivery to the vagina: a review. $J$ Control Release 190, 500-514, doi:10.1016/j.jconrel.2014.04.033 (2014).

189 Ahmad, M. Z. et al. Metallic nanoparticles: technology overview \& drug delivery applications in oncology. Expert Opin Drug Del 7, 927-942, doi:10.1517/17425247.2010.498473 (2010).

190 Mody, V. V., Siwale, R., Singh, A. \& Mody, H. R. Introduction to metallic nanoparticles. J Pharm Bioallied Sci 2, 282-289, doi:10.4103/0975-7406.72127 (2010).

191 Singh, R. \& Lillard, J. W. Nanoparticle-based targeted drug delivery. Exp Mol Patho/ 86, 215-223, doi:10.1016/j.yexmp.2008.12.004 (2009).

192 Blanco, E., Shen, H. \& Ferrari, M. Principles of nanoparticle design for overcoming biological barriers to drug delivery. Nat Biotechnol 33, 941-951, doi:10.1038/nbt.3330 (2015).

193 Peer, D. et al. Nanocarriers as an emerging platform for cancer therapy. Nat Nanotechnol 2, 751-760, doi:10.1038/nnano.2007.387 (2007).

194 Arias, J. L. Nanotechnology and Drug Delivery, Volume Two. (2016).

195 Gu, J. J., Yang, S. D. \& Ho, E. A. Biodegradable Film for the Targeted Delivery of siRNA-Loaded Nanoparticles to Vaginal Immune Cells. Mol Pharmaceut 12, 28892903, doi:10.1021/acs.molpharmaceut.5b00073 (2015).

196 Wang, Y. Y. et al. Addressing the PEG Mucoadhesivity Paradox to Engineer Nanoparticles that "Slip" through the Human Mucus Barrier. Angew Chem Int Edit 47, 9726-9729, doi:10.1002/anie.200803526 (2008).

197 Wang, S., Zhao, Y., Shen, M. \& Shi, X. Electrospun hybrid nanofibers doped with nanoparticles or nanotubes for biomedical applications. Ther Deliv 3, 1155-1169 (2012).

198 Chen, M. L. et al. Chitosan/siRNA Nanoparticles Encapsulated in PLGA Nanofibers for siRNA Delivery. Acs Nano 6, 4835-4844, doi:10.1021/nn300106t (2012).

199 Sridhar, R. et al. Electrosprayed nanoparticles and electrospun nanofibers based on natural materials: applications in tissue regeneration, drug delivery and pharmaceuticals. Chem Soc Rev 44, 790-814, doi:10.1039/c4cs00226a (2015).

200 Mehrasa, M. et al. Incorporation of mesoporous silica nanoparticles into random electrospun PLGA and PLGA/gelatin nanofibrous scaffolds enhances mechanical and cell proliferation properties. Mat Sci Eng C-Mater 66, 25-32, doi:10.1016/j.msec.2016.04.031 (2016).

201 Song, B. T., Wu, C. T. \& Chang, J. Dual drug release from electrospun poly(lacticco-glycolic acid)/mesoporous silica nanoparticles composite mats with distinct 
release profiles. Acta Biomater 8, 1901-1907, doi:10.1016/j.actbio.2012.01.020 (2012).

202 Beck-Broichsitter, M. et al. Novel 'Nano in Nano' Composites for Sustained Drug Delivery: Biodegradable Nanoparticles Encapsulated into Nanofiber Non-Wovens. Macromol Biosci 10, 1527-1535, doi:10.1002/mabi.201000100 (2010).

203 Fathollahipour, S., Mehrizi, A. A., Ghaee, A. \& Koosha, M. Electrospinning of PVA/chitosan nanocomposite nanofibers containing gelatin nanoparticles as a dual drug delivery system. J Biomed Mater Res A 103, 3852-3862, doi:10.1002/jbm.a.35529 (2015).

204 Hu, J., Zeng, F., Wei, J., Chen, Y. \& Chen, Y. Novel controlled drug delivery system for multiple drugs based on electrospun nanofibers containing nanomicelles. $J$ Biomater Sci Polym Ed 25, 257-268, doi:10.1080/09205063.2013.852367 (2014).

205 Wang, Y. Z., Wang, B. C., Qiao, W. L. \& Yin, T. Y. A Novel Controlled Release Drug Delivery System for Multiple Drugs Based on Electrospun Nanofibers Containing Nanoparticles. J Pharm Sci-Us 99, 4805-4811, doi:10.1002/jps.22189 (2010).

$206 \mathrm{Li}$, L. et al. Controlled dual delivery of BMP-2 and dexamethasone by nanoparticleembedded electrospun nanofibers for the efficient repair of critical-sized rat calvarial defect. Biomaterials 37, 218-229, doi:10.1016/j.biomaterials.2014.10.015 (2015).

207 Ali, I. H., Khalil, I. A. \& El-Sherbiny, I. M. Single-Dose Electrospun Nanoparticlesin-Nanofibers Wound Dressings with Enhanced Epithelialization, Collagen Deposition, and Granulation Properties. Acs Appl Mater Inter 8, 14453-14469, doi:10.1021/acsami.6b04369 (2016).

208 Sun, X. et al. Rationally designed particle preloading method to improve protein delivery performance of electrospun polyester nanofibers. Int $J$ Pharm 512, 204$212(2016)$.

209 Vakilian, S. et al. Structural stability and sustained release of protein from a multilayer nanofiber/nanoparticle composite. Int J Biol Macromol 75, 248-257, doi:10.1016/j.jibiomac.2015.01.051 (2015).

210 Nie, H. M. \& Wang, C. H. Fabrication and characterization of PLGA/HAp scaffolds for delivery of BMP-2 plasmid composite DNA. Journal of Controlled Release 120, 111-121, doi:10.1016/j.jconrel.2007.03.018 (2007).

211 Cui, W., Zhou, Y. \& Chang, J. Electrospun nanofibrous materials for tissue engineering and drug delivery. Sci Technol Adv Mater 11, 014108, doi:10.1088/1468-6996/11/1/014108 (2010).

212 Shao, W. L. et al. Coaxial electrospun aligned tussah silk fibroin nanostructured fiber scaffolds embedded with hydroxyapatite-tussah silk fibroin nanoparticles for bone tissue engineering. Mat Sci Eng C-Mater 58, 342-351, doi:10.1016/j.msec.2015.08.046 (2016).

213 Weissleder, R., Kelly, K., Sun, E. Y., Shtatland, T. \& Josephson, L. Cell-specific targeting of nanoparticles by multivalent attachment of small molecules. Nat Biotechnol 23, 1418-1423, doi:10.1038/nbt1159 (2005).

214 Kohler, N., Fryxell, G. E. \& Zhang, M. Q. A bifunctional poly(ethylene glycol) silane immobilized on metallic oxide-based nanoparticles for conjugation with cell targeting agents. J Am Chem Soc 126, 7206-7211, doi:DOI 10.1021/ja049195r (2004).

215 Krogstad, E. A. et al. Nanoparticle-releasing nanofiber composites for enhanced in vivo vaginal retention. Biomaterials 144, 1-16, doi:10.1016/j.biomaterials.2017.07.034 (2017). 
216 Yao, L., Lin, Y. \& Watkins, J. J. Ultrahigh Loading of Nanoparticles into Ordered Block Copolymer Composites. Macromolecules 47, 1844-1849, doi:10.1021/ma500338p (2014).

217 Zhu, J. H. et al. Electrospun Polyimide Nanocomposite Fibers Reinforced with Core-Shell Fe-FeO Nanoparticles. J Phys Chem C 114, 8844-8850, doi:10.1021/jp1020033 (2010).

218 Delany-Moretlwe, S. et al. Tenofovir 1\% vaginal gel for prevention of HIV-1 infection in women in South Africa (FACTS-001): a phase 3, randomised, doubleblind, placebo-controlled trial. Lancet Infect Dis 18, 1241-1250, doi:10.1016/S1473-3099(18)30428-6 (2018).

219 Skoler-Karpoff, S. et al. Efficacy of Carraguard for prevention of HIV infection in women in South Africa: a randomised, double-blind, placebo-controlled trial. The Lancet 372, 1977-1987, doi:https://doi.org/10.1016/S0140-6736(08)61842-5 (2008).

220 Thurman, A. R., Clark, M. R. \& Doncel, G. F. Multipurpose prevention technologies: biomedical tools to prevent HIV-1, HSV-2, and unintended pregnancies. Infect Dis Obstet Gynecol 2011, 1-10, doi:10.1155/2011/429403 (2011).

221 Blakney, A. K., Simonovsky, F. I., Suydam, I. T., Ratner, B. D. \& Woodrow, K. A. Rapidly Biodegrading PLGA-Polyurethane Fibers for Sustained Release of Physicochemically Diverse Drugs. ACS Biomater Sci Eng 2, 1595-1607, doi:10.1021/acsbiomaterials.6b00346 (2016).

222 Halwes, M. E., Tyo, K. M., Steinbach-Rankins, J. M. \& Frieboes, H. B. Computational Modeling of Antiviral Drug Diffusion from Poly(lactic- co-glycolicacid) Fibers and Multicompartment Pharmacokinetics for Application to the Female Reproductive Tract. Mol Pharm 15, 1534-1547, doi:10.1021/acs.molpharmaceut.7b01089 (2018).

223 Moss, J. A. et al. Pharmacokinetics of a multipurpose pod-intravaginal ring simultaneously delivering five drugs in an ovine model. Antimicrob Agents Chemother 57, 3994-3997, doi:10.1128/AAC.00547-13 (2013).

224 Smith, J. M. et al. Novel multipurpose pod-intravaginal ring for the prevention of HIV, HSV, and unintended pregnancy: Pharmacokinetic evaluation in a macaque model. PLoS One 12, e0185946, doi:10.1371/journal.pone.0185946 (2017).

225 Morrow, R. J. et al. Sustained release of proteins from a modified vaginal ring device. Eur J Pharm Biopharm 77, 3-10, doi:10.1016/j.ejpb.2010.10.010 (2011).

226 Han, Y. A., Singh, M. \& Saxena, B. B. Development of vaginal rings for sustained release of nonhormonal contraceptives and anti-HIV agents. Contraception 76, 132-138, doi:10.1016/j.contraception.2007.04.006 (2007).

227 Malcolm, R. K. et al. Sustained release of the CCR5 inhibitors CMPD167 and maraviroc from vaginal rings in rhesus macaques. Antimicrob Agents Chemother 56, 2251-2258, doi:10.1128/AAC.05810-11 (2012).

228 Johnson, T. J., Gupta, K. M., Fabian, J., Albright, T. H. \& Kiser, P. F. Segmented polyurethane intravaginal rings for the sustained combined delivery of antiretroviral agents dapivirine and tenofovir. Eur $J$ Pharm Sci 39, 203-212, doi:10.1016/j.ejps.2009.11.007 (2010).

229 Malcolm, R. K., Woolfson, A. D., Toner, C. F., Morrow, R. J. \& McCullagh, S. D. Long-term, controlled release of the HIV microbicide TMC120 from silicone elastomer vaginal rings. J Antimicrob Chemother 56, 954-956, doi:10.1093/jac/dki326 (2005).

230 Baum, M. M. et al. An intravaginal ring for the simultaneous delivery of multiple drugs. J Pharm Sci 101, 2833-2843, doi:10.1002/jps.23208 (2012). 
231 Johnson, T. J. et al. A 90-day tenofovir reservoir intravaginal ring for mucosal HIV prophylaxis. Antimicrob Agents Chemother 56, 6272-6283, doi:10.1128/AAC.01431-12 (2012).

232 Blakney, A. K., Little, A. B., Jiang, Y. \& Woodrow, K. A. In vitro-ex vivo correlations between a cell-laden hydrogel and mucosal tissue for screening composite delivery systems. Drug Deliv 24, 582-590, doi:10.1080/10717544.2016.1242178 (2016).

233 Rohan, L. C. et al. In vitro and ex vivo testing of tenofovir shows it is effective as an HIV-1 microbicide. PLoS One 5, e9310, doi:10.1371/journal.pone.0009310 (2010).

234 Patton, D. L. et al. Preclinical safety assessments of UC781 anti-human immunodeficiency virus topical microbicide formulations. Antimicrob Agents Chemother 51, 1608-1615, doi:10.1128/AAC.00984-06 (2007).

235 Robinson, J. A. et al. Comparison of the Pharmacokinetics and Pharmacodynamics of Single-Dose Tenofovir Vaginal Film and Gel Formulation (FAME 05). J Acquir Immune Defic Syndr 77, 175-182, doi:10.1097/QAI.0000000000001587 (2018).

236 Hu, M., Zhou, T., Dezzutti, C. S. \& Rohan, L. C. The Effect of Commonly Used Excipients on the Epithelial Integrity of Human Cervicovaginal Tissue. AIDS Res Hum Retroviruses 32, 992-1004, doi:10.1089/AID.2016.0014 (2016).

237 Merbah, M. et al. Cervico-vaginal tissue ex vivo as a model to study early events in HIV-1 infection. Am J Reprod Immunol 65, 268-278, doi:10.1111/j.16000897.2010.00967.x (2011).

238 Ayehunie, S. et al. Organotypic human vaginal-ectocervical tissue model for irritation studies of spermicides, microbicides, and feminine-care products. Toxicol In Vitro 20, 689-698, doi:10.1016/j.tiv.2005.10.002 (2006).

239 Ayehunie, S. et al. Development of an in vitro alternative assay method for vaginal irritation. Toxicology 279, 130-138, doi:10.1016/j.tox.2010.10.001 (2011).

240 Laniewski, P., Gomez, A., Hire, G., So, M. \& Herbst-Kralovetz, M. M. Human Three-Dimensional Endometrial Epithelial Cell Model To Study Host Interactions with Vaginal Bacteria and Neisseria gonorrhoeae. Infect Immun 85, doi:10.1128/IAI.01049-16 (2017).

241 Doncel, G. F. \& Clark, M. R. Preclinical evaluation of anti-HIV microbicide products: New models and biomarkers. Antiviral Res 88 Suppl 1, S10-18, doi:10.1016/j.antiviral.2010.09.018 (2010).

242 Krogstad, E. A. \& Woodrow, K. A. Manufacturing scale-up of electrospun poly(vinyl alcohol) fibers containing tenofovir for vaginal drug delivery. Int J Pharm 475, 282291, doi:10.1016/j.ijpharm.2014.08.039 (2014).

243 Jiang, J. et al. Mussel-inspired protein-mediated surface functionalization of electrospun nanofibers for pH-responsive drug delivery. Acta Biomater 10, 13241332, doi:10.1016/j.actbio.2013.11.012 (2014).

244 Sun, X. Z., Williams, G. R., Hou, X. X. \& Zhu, L. M. Electrospun curcumin-loaded fibers with potential biomedical applications. Carbohydr Polym 94, 147-153, doi:10.1016/j.carbpol.2012.12.064 (2013).

245 Berg, M. C., Zhai, L., Cohen, R. E. \& Rubner, M. F. Controlled drug release from porous polyelectrolyte multilayers. Biomacromolecules 7, 357-364, doi:10.1021/bm050174e (2006).

246 Owen, D. H. \& Katz, D. F. A vaginal fluid simulant. Contraception 59, 91-95 (1999).

247 Wei, X. et al. Emergence of resistant human immunodeficiency virus type 1 in patients receiving fusion inhibitor (T-20) monotherapy. Antimicrob Agents Chemother 46, 1896-1905, doi:10.1128/aac.46.6.1896-1905.2002 (2002). 
248 Platt, E. J., Wehrly, K., Kuhmann, S. E., Chesebro, B. \& Kabat, D. Effects of CCR5 and CD4 cell surface concentrations on infections by macrophagetropic isolates of human immunodeficiency virus type 1. J Virol 72, 2855-2864 (1998).

249 Montefiori, D. C. Measuring HIV neutralization in a luciferase reporter gene assay. Methods Mol Biol 485, 395-405, doi:10.1007/978-1-59745-170-3_26 (2009).

250 Ham, A. S. et al. Development of a combination microbicide gel formulation containing IQP-0528 and tenofovir for the prevention of HIV infection. J Pharm Sci 101, 1423-1435, doi:10.1002/jps.23026 (2012).

251 Fichorova, R. N. et al. A quantitative multiplex nuclease protection assay reveals immunotoxicity gene expression profiles in the rabbit model for vaginal drug safety evaluation. Toxicol Appl Pharmacol 285, 198-206, doi:10.1016/j.taap.2015.02.017 (2015).

252 Nixon, B. et al. Vaginally delivered tenofovir disoproxil fumarate provides greater protection than tenofovir against genital herpes in a murine model of efficacy and safety. Antimicrob Agents Chemother 58, 1153-1160, doi:10.1128/AAC.01818-13 (2014).

253 Chen, S. C. et al. The Influence of Fiber Diameter of Electrospun Poly(lactic acid) on Drug Delivery. Fiber Polym 13, 1120-1125, doi:10.1007/s12221-012-1120-x (2012).

254 Hrib, J. et al. Nanofibers for drug delivery - incorporation and release of model molecules, influence of molecular weight and polymer structure. Beilstein $J$ Nanotechnol 6, 1939-1945, doi:10.3762/bjnano.6.198 (2015).

255 Chew, S. Y., Hufnagel, T. C., Lim, C. T. \& Leong, K. W. Mechanical properties of single electrospun drug-encapsulated nanofibres. Nanotechnology 17, 3880-3891, doi:10.1088/0957-4484/17/15/045 (2006).

256 Zong, X. et al. Structure and process relationship of electrospun bioabsorbable nanofiber membranes. Polymer 43, 4403-4412, doi:https://doi.org/10.1016/S00323861(02)00275-6 (2002).

257 DrugBank. Tenofovir, <https://www.drugbank.ca/drugs/DB00300> (2005).

258 DrugBank. Tenofovir Disoproxil Fumarate, <https://www.drugbank.ca/salts/DBSALT000172> (2005).

$259 \mathrm{Gu}, \mathrm{X} . \mathrm{H}$. et al. Electrospinning of poly(butylene-carbonate): Effect of Solvents on the Properties of the Nanofibers Film. Int J Electrochem Sc 9, 8045-8056 (2014).

260 Sun, Z. C., Deitzel, J. M., Knopf, J., Chen, X. \& Gillespie, J. W. The effect of solvent dielectric properties on the collection of oriented electrospun fibers. Journal of Applied Polymer Science 125, 2585-2594, doi:10.1002/app.35454 (2012).

261 Aldrich, $\quad$ S. 2,2,2-Trifluoroethanol ReagentPlus $\AA, \quad \geq 99 \%$ $<$ https://www.sigmaaldrich.com/catalog/product/sigald/t63002?lang=en\&region= US> (

262 Aldrich, S. 1,1,1,3,3,3-Hexafluoro-2-propanol $\quad 299 \%$ $<$ https://www.sigmaaldrich.com/catalog/product/aldrich/105228?lang=en\&region= US> (

263 Yu, D.-G., Zhu, L.-M., White, K. \& Branford-White, C. Electrospun nanofiber-based drug delivery systems. Health Vol.01No.02, 9, doi:10.4236/health.2009.12012 (2009).

264 Huang, Z. M. et al. Encapsulating drugs in biodegradable ultrafine fibers through co-axial electrospinning. J Biomed Mater Res A 77, 169-179, doi:10.1002/jbm.a.30564 (2006).

265 Geboers, S. et al. Intestinal behavior of the ester prodrug tenofovir DF in humans. Int J Pharm 485, 131-137, doi:https://doi.org/10.1016/j.ijpharm.2015.03.002 (2015). 
266 Galvin, S. R. \& Cohen, M. S. The role of sexually transmitted diseases in HIV transmission. Nat Rev Microbiol 2, 33-42, doi:10.1038/nrmicro794 (2004).

267 Havele, S. \& Dhaneshwar, S. R. Stress studies of tenofovir disoproxil fumarate by HPTLC in bulk drug and pharmaceutical formulation. ScientificWorldJournal 2012, 894136-894136, doi:10.1100/2012/894136 (2012).

268 Agrahari, V., Putty, S., Mathes, C., Murowchick, J. B. \& Youan, B. B. C. Evaluation of degradation kinetics and physicochemical stability of tenofovir. Drug Test Anal 7, 207-213, doi:10.1002/dta.1656 (2015).

269 Doncel, G. F., Chandra, N. \& Fichorova, R. N. Preclinical assessment of the proinflammatory potential of microbicide candidates. J Acquir Immune Defic Syndr 37 Suppl 3, S174-180 (2004).

270 Dayal, M. B., Wheeler, J., Williams, C. J. \& Barnhart, K. T. Disruption of the upper female reproductive tract epithelium by nonoxynol-9. Contraception 68, 273-279 (2003).

271 Fichorova, R. N., Tucker, L. D. \& Anderson, D. J. The molecular basis of nonoxynol-9-induced vaginal inflammation and its possible relevance to human immunodeficiency virus type 1 transmission. J Infect Dis 184, 418-428, doi:10.1086/322047 (2001).

272 Jain, J. K., Li, A., Minoo, P., Nucatola, D. L. \& Felix, J. C. The effect of nonoxynol9 on human endometrium. Contraception 71, 137-142, doi:10.1016/j.contraception.2004.08.012 (2005).

273 Montgomery, E. T. et al. Misreporting of Product Adherence in the MTN003/VOICE Trial for HIV Prevention in Africa: Participants' Explanations for Dishonesty. AIDS Behav 21, 481-491, doi:10.1007/s10461-016-1609-1 (2017).

274 Woodsong, C. et al. Microbicide clinical trial adherence: insights for introduction. $J$ Int AIDS Soc 16, 18505, doi:10.7448/IAS.16.1.18505 (2013).

275 IPM. Identifying Women's Needs and Preferences, $<$ https://www.ipmglobal.org/our-work/access/identifying-women-needspreferences $>$ (2017).

276 Fan, M. D. et al. Preferred Physical Characteristics of Vaginal Film Microbicides for HIV Prevention in Pittsburgh Women. Arch Sex Behav 46, 1111-1119, doi:10.1007/s10508-016-0816-1 (2017).

277 Zhou, K. J. et al. Tunable, Ultrasensitive pH-Responsive Nanoparticles Targeting Specific Endocytic Organelles in Living Cells. Angew Chem Int Edit 50, 6109-6114, doi:10.1002/anie.201100884 (2011).

278 Gao, W., Chan, J. M. \& Farokhzad, O. C. pH-Responsive nanoparticles for drug delivery. Mol Pharm 7, 1913-1920, doi:10.1021/mp100253e (2010).

279 Huang, C. et al. Stimuli-responsive electrospun fibers and their applications. Chem Soc Rev 40, 2417-2434, doi:10.1039/c0cs00181c (2011).

280 Yoo, J. W., Giri, N. \& Lee, C. H. pH-sensitive Eudragit nanoparticles for mucosal drug delivery. Int $J$ Pharm 403, 262-267, doi:10.1016/j.jpharm.2010.10.032 (2011).

281 Goh, Y. F., Shakir, I. \& Hussain, R. Electrospun fibers for tissue engineering, drug delivery, and wound dressing. J Mater Sci 48, 3027-3054, doi:10.1007/s10853013-7145-8 (2013).

282 Gupta, K. M. et al. Temperature and pH sensitive hydrogels: an approach towards smart semen-triggered vaginal microbicidal vehicles. J Pharm Sci 96, 670-681, doi:10.1002/jps.20752 (2007).

283 Zhang, T., Sturgis, T. F. \& Youan, B. B. pH-responsive nanoparticles releasing tenofovir intended for the prevention of HIV transmission. Eur J Pharm Biopharm 79, 526-536, doi:10.1016/j.ejpb.2011.06.007 (2011). 
284 Zhang, T. et al. Spray drying tenofovir loaded mucoadhesive and $\mathrm{pH}$-sensitive microspheres intended for HIV prevention. Antiviral Research 97, 334-346, doi:10.1016/j.antiviral.2012.12.019 (2013).

285 Mahalingam, A. et al. Inhibition of the transport of HIV in vitro using a pHresponsive synthetic mucin-like polymer system. Biomaterials 32, 8343-8355, doi:10.1016/j.biomaterials.2011.05.001 (2011).

286 Frizzell, H., Ohlsen, T. J. \& Woodrow, K. A. Protein-loaded emulsion electrospun fibers optimized for bioactivity retention and $\mathrm{pH}$-controlled release for peroral delivery of biologic therapeutics. Int $J$ Pharm 533, 99-110, doi:10.1016/j.ijpharm.2017.09.043 (2017).

287 Nie, H. W., K. Society for Biomaterials (2014).

288 Philippova, O. E., Hourdet, D., Audebert, R. \& Khokhlov, A. R. pH-responsive gels of hydrophobically modified poly(acrylic acid). Macromolecules 30, 8278-8285, doi:DOl 10.1021/ma970957v (1997).

289 Gupta, P., Vermani, K. \& Garg, S. Hydrogels: from controlled release to pHresponsive drug delivery. Drug Discov Today 7, 569-579 (2002).

290 de Vries, M. E., Bodde, H. E., Busscher, H. J. \& Junginger, H. E. Hydrogels for buccal drug delivery: properties relevant for muco-adhesion. J Biomed Mater Res 22, 1023-1032, doi:10.1002/jbm.820221106 (1988).

291 Sood, N., Bhardwaj, A., Mehta, S. \& Mehta, A. Stimuli-responsive hydrogels in drug delivery and tissue engineering. Drug Deliv 23, 758-780, doi:10.3109/10717544.2014.940091 (2016).

292 Jin, X. \& Hsieh, Y. L. pH-responsive swelling behavior of poly(vinyl alcohol)/poly(acrylic acid) bi-component fibrous hydrogel membranes. Polymer 46, 5149-5160, doi:10.1016/j.polymer.2005.04.066 (2005).

293 Sant, V. P., Smith, D. \& Leroux, J. C. Enhancement of oral bioavailability of poorly water-soluble drugs by poly(ethylene glycol)-block-poly(alkyl acrylate-comethacrylic acid) self-assemblies. $J$ Control Release 104, 289-300, doi:10.1016/j.jconrel.2005.02.010 (2005).

294 Liu, F. \& Urban, M. W. Dual Temperature and pH Responsiveness of Poly(2-(N,Ndimethylamino)ethyl methacrylate-co-n-butyl acrylate) Colloidal Dispersions and Their Films. Macromolecules 41, 6531-6539, doi:10.1021/ma8006784 (2008).

295 Bodde, H. E., Devries, M. E. \& Junginger, H. E. Mucoadhesive Polymers for the Buccal Delivery of Peptides, Structure Adhesiveness Relationships. Journal of Controlled Release 13, 225-231, doi:Doi 10.1016/0168-3659(90)90012-I (1990).

296 Owen, D. H. \& Katz, D. F. A review of the physical and chemical properties of human semen and the formulation of a semen simulant. $J$ Androl 26, 459-469, doi:10.2164/jandrol.04104 (2005).

297 Sarzotti-Kelsoe, M. et al. Optimization and validation of the TZM-bl assay for standardized assessments of neutralizing antibodies against HIV-1. J Immunol Methods 409, 131-146, doi:10.1016/j.jim.2013.11.022 (2014).

298 Yang, L. et al. Mechanical properties of single electrospun collagen type I fibers. Biomaterials 29, 955-962, doi:10.1016/j.biomaterials.2007.10.058 (2008).

299 Chew, S. Y., Wen, J., Yim, E. K. \& Leong, K. W. Sustained release of proteins from electrospun biodegradable fibers. Biomacromolecules 6, 2017-2024, doi:10.1021/bm0501149 (2005).

300 Jensen, S. M. et al. Differential inhibitory effects of cyanovirin-N, griffithsin, and scytovirin on entry mediated by envelopes of gammaretroviruses and deltaretroviruses. J Virol 88, 2327-2332, doi:10.1128/JVI.02553-13 (2014).

301 Mitchell, C. A., Ramessar, K. \& O'Keefe, B. R. Antiviral lectins: Selective inhibitors of viral entry. Antiviral Res 142, 37-54, doi:10.1016/j.antiviral.2017.03.007 (2017). 
302 Ducheyne, P. Comprehensive Biomaterials. Vol. 1 (Elsevier, 2011).

303 Pan, Z. \& Ding, J. Poly(lactide-co-glycolide) porous scaffolds for tissue engineering and regenerative medicine. Interface Focus 2, 366-377, doi:10.1098/rsfs.2011.0123 (2012).

304 Sutton, D., Durand, R., Shuai, X. T. \& Gao, J. M. Poly(D, L-lactide-coglycolide)/poly(ethylenimine) blend matrix system for $\mathrm{pH}$ sensitive drug delivery. Journal of Applied Polymer Science 100, 89-96, doi:10.1002/app.22636 (2006).

305 Colombani, O. et al. Structure of micelles of poly(n-butyl acrylate)-block-poly (acrylic acid) diblock copolymers in aqueous solution. Macromolecules 40, 43514362, doi:10.1021/ma0609580 (2007).

306 Sant, V. P., Smith, D. \& Leroux, J. C. Novel pH-sensitive supramolecular assemblies for oral delivery of poorly water soluble drugs: preparation and characterization. Journal of Controlled Release 97, 301-312, doi:10.1016/j.jconrel.2004.03.026 (2004).

307 Garbern, J. C., Hoffman, A. S. \& Stayton, P. S. Injectable pH- and temperatureresponsive poly( $\mathrm{N}$-isopropylacrylamide-co-propylacrylic acid) copolymers for delivery of angiogenic growth factors. Biomacromolecules 11, 1833-1839, doi:10.1021/bm100318z (2010).

308 Dong, L. C., Yan, Q. \& Hoffman, A. S. Controlled Release of Amylase from a Thermal and Ph-Sensitive, Macroporous Hydrogel. Journal of Controlled Release 19, 171-177 (1992).

309 Kim, Y. H., Bae, Y. H. \& Kim, S. W. Ph/Temperature-Sensitive Polymers for Macromolecular Drug Loading and Release. Journal of Controlled Release 28, 143-152 (1994).

310 Chen, G. \& Hoffman, A. S. Graft copolymers that exhibit temperature-induced phase transitions over a wide range of $\mathrm{pH}$. Nature 373 , 49-52, doi:10.1038/373049a0 (1995).

311 Bilia, A., Carelli, V., DiColo, G. \& Nannipieri, E. In vitro evaluation of a pH-sensitive hydrogel for control of GI drug delivery from silicone-based matrices. Int $J$ Pharm 130, 83-92, doi:Doi 10.1016/0378-5173(95)04297-0 (1996).

312 Shaikh, R., Raj Singh, T. R., Garland, M. J., Woolfson, A. D. \& Donnelly, R. F. Mucoadhesive drug delivery systems. J Pharm Bioallied Sci 3, 89-100, doi:10.4103/0975-7406.76478 (2011).

313 Marques, M. R. C., Loebenberg, R. \& Almukainzi, M. Simulated Biological Fluids with Possible Application in Dissolution Testing. Dissolut Technol 18, 15-28, doi:Doi 10.14227/Dt180311p15 (2011).

314 Beumer, G. J., van Blitterswijk, C. A., Bakker, D. \& Ponec, M. Cell-seeding and in vitro biocompatibility evaluation of polymeric matrices of PEO/PBT copolymers and PLLA. Biomaterials 14, 598-604, doi:https://doi.org/10.1016/01429612(93)90178-5 (1993).

315 Hago, E.-E. \& Li, X. Interpenetrating Polymer Network Hydrogels Based on Gelatin and PVA by Biocompatible Approaches: Synthesis and Characterization. Advances in Materials Science and Engineering 2013, 8, doi:10.1155/2013/328763 (2013).

316 Singh, B. \& Pal, L. Sterculia crosslinked PVA and PVA-poly(AAm) hydrogel wound dressings for slow drug delivery: Mechanical, mucoadhesive, biocompatible and permeability properties. Journal of the Mechanical Behavior of Biomedical Materials 9, 9-21, doi:https://doi.org/10.1016/j.jmbbm.2012.01.021 (2012).

317 Rogero, S. O. et al. Biocompatibility Study of Polymeric Biomaterials. Artificial Organs 27, 424-427, doi:10.1046/j.1525-1594.2003.07249.x (2003). 
318 Hendrickson, B. A. et al. Decreased Vaginal Disease in J-Chain-Deficient Mice Following Herpes Simplex Type 2 Genital Infection. Virology 271, 155-162, doi:https://doi.org/10.1006/viro.2000.0303 (2000).

319 Yang, $H$. et al. Design of Poly(lactic-co-glycolic Acid) (PLGA) Nanoparticles for Vaginal Co-Delivery of Griffithsin and Dapivirine and Their Synergistic Effect for HIV Prophylaxis. Pharmaceutics 11, 184 (2019).

320 Valenta, C. The use of mucoadhesive polymers in vaginal delivery. Adv Drug Deliver Rev 57, 1692-1712, doi:https://doi.org/10.1016/j.addr.2005.07.004 (2005).

321 Apicella, A. et al. Poly(Ethylene oxide) (PEO) and different molecular weight PEO blends monolithic devices for drug release. Biomaterials 14, 83-90, doi:https://doi.org/10.1016/0142-9612(93)90215-N (1993).

$322 \mathrm{Li}, \mathrm{J}$. et al. Self-assembled supramolecular hydrogels formed by biodegradable PEO-PHB-PEO triblock copolymers and a-cyclodextrin for controlled drug delivery. Biomaterials 27, 4132-4140, doi:https://doi.org/10.1016/j.biomaterials.2006.03.025 (2006).

323 Jeong, B., Choi, Y. K., Bae, Y. H., Zentner, G. \& Kim, S. W. New biodegradable polymers for injectable drug delivery systems. Journal of Controlled Release 62, 109-114, doi:https://doi.org/10.1016/S0168-3659(99)00061-9 (1999).

324 Desai, S. D. \& Blanchard, J. In Vitro Evaluation of Pluronic F127-Based ControlledRelease Ocular Delivery Systems for Pilocarpine. J Pharm Sci-Us 87, 226-230, doi:https://doi.org/10.1021/js970090e (1998).

325 Wang, L., Chang, M.-W., Ahmad, Z., Zheng, H. \& Li, J.-S. Mass and controlled fabrication of aligned PVP fibers for matrix type antibiotic drug delivery systems. Chemical Engineering Journal 307, 661-669, doi:https://doi.org/10.1016/j.cej.2016.08.135 (2017).

326 Pal, K., Banthia, A. K. \& Majumdar, D. K. Preparation and characterization of polyvinyl alcohol-gelatin hydrogel membranes for biomedical applications. AAPS PharmSciTech 8, E142-E146, doi:10.1208/pt080121 (2007).

327 Li, X., Kanjwal, M. A., Lin, L. \& Chronakis, I. S. Electrospun polyvinyl-alcohol nanofibers as oral fast-dissolving delivery system of caffeine and riboflavin. Colloids and Surfaces B: Biointerfaces 103, 182-188, doi:https://doi.org/10.1016/j.colsurfb.2012.10.016 (2013).

328 Jeong, B., Bae, Y. H., Lee, D. S. \& Kim, S. W. Biodegradable block copolymers as injectable drug-delivery systems. Nature 388, 860-862, doi:10.1038/42218 (1997).

329 Wen, P., Wen, Y., Zong, M. H., Linhardt, R. J. \& Wu, H. Encapsulation of Bioactive Compound in Electrospun Fibers and Its Potential Application. J Agr Food Chem 65, 9161-9179, doi:10.1021/acs.jafc.7b02956 (2017).

330 Seif, S., Planz, V. \& Windbergs, M. Delivery of Therapeutic Proteins Using Electrospun Fibers-Recent Developments and Current Challenges. Arch Pharm 350, doi:ARTN e170007710.1002/ardp.201700077 (2017).

331 Vermani, K. \& Garg, S. The scope and potential of vaginal drug delivery. Pharmaceutical Science \& Technology Today 3, 359-364, doi:https://doi.org/10.1016/S1461-5347(00)00296-0 (2000).

332 Keefe, B. R. et al. Broad-Spectrum \&lt;em\&gt;In Vitro\&lt;/em\&gt; Activity and \&lt;em\&gt; In Vivo\&lt;/em\&gt; Efficacy of the Antiviral Protein Griffithsin against Emerging Viruses of the Family \&lt;em\&gt;Coronaviridae\&lt;/em\&gt. Journal of Virology 84, 2511, doi:10.1128/JVI.02322-09 (2010).

$333 \mathrm{Ji}, \mathrm{W}$. et al. Biocompatibility and degradation characteristics of PLGA-based electrospun nanofibrous scaffolds with nanoapatite incorporation. Biomaterials $\mathbf{3 3}$, 6604-6614, doi:10.1016/j.biomaterials.2012.06.018 (2012). 
334 Machado, A. et al. Development and in vivo safety assessment of tenofovir-loaded nanoparticles-in-film as a novel vaginal microbicide delivery system. Acta Biomater 44, 332-340, doi:10.1016/j.actbio.2016.08.018 (2016).

335 Haider, A., Haider, S. \& Kang, I. K. A comprehensive review summarizing the effect of electrospinning parameters and potential applications of nanofibers in biomedical and biotechnology. Arab $J$ Chem 11, 1165-1188, doi:10.1016/j.arabjc.2015.11.015 (2018).

336 Oppenheim, J. J. Cytokine Reference: a compendium of cytokines and other mediators of host defense. 1.[Ligands]. (Academic Press, 2001).

337 Schindler, R. et al. Correlations and interactions in the production of interleukin-6 (IL-6), IL-1, and tumor necrosis factor (TNF) in human blood mononuclear cells: IL-6 suppresses IL-1 and TNF. Blood 75, 40-47 (1990).

338 Matsukawa, A. et al. Endogenous MCP-1 Influences Systemic Cytokine Balance in a Murine Model of Acute Septic Peritonitis. Exp Mol Pathol 68, 77-84, doi:https://doi.org/10.1006/exmp.1999.2296 (2000).

339 Deshmane, S. L., Kremlev, S., Amini, S. \& Sawaya, B. E. Monocyte chemoattractant protein-1 (MCP-1): an overview. J Interferon Cytokine Res 29, 313-326, doi:10.1089/jir.2008.0027 (2009).

340 Cook, D. N. The role of MIP-1a in Inflammation and hematopoiesis. Journal of Leukocyte Biology 59, 61-66, doi:10.1002/jlb.59.1.61 (1996).

341 Catalone, B. J. et al. Mouse model of cervicovaginal toxicity and inflammation for preclinical evaluation of topical vaginal microbicides. Antimicrobial agents and chemotherapy 48, 1837-1847, doi:10.1128/aac.48.5.1837-1847.2004 (2004).

342 Blakney, A. K., Jiang, Y. \& Woodrow, K. A. Application of electrospun fibers for female reproductive health. Drug Deliv Transl Res 7, 796-804, doi:10.1007/s13346-017-0386-3 (2017).

343 Soppimath, K. S., Aminabhavi, T. M., Kulkarni, A. R. \& Rudzinski, W. E. Biodegradable polymeric nanoparticles as drug delivery devices. Journal of Controlled Release 70, 1-20, doi:https://doi.org/10.1016/S0168-3659(00)00339-4 (2001).

344 Kumari, A., Yadav, S. K. \& Yadav, S. C. Biodegradable polymeric nanoparticles based drug delivery systems. Colloids and Surfaces B: Biointerfaces 75, 1-18, doi:https://doi.org/10.1016/j.colsurfb.2009.09.001 (2010).

345 Hans, M. L. \& Lowman, A. M. Biodegradable nanoparticles for drug delivery and targeting. Current Opinion in Solid State and Materials Science 6, 319-327, doi:https://doi.org/10.1016/S1359-0286(02)00117-1 (2002).

346 Panyam, J. \& Labhasetwar, V. Biodegradable nanoparticles for drug and gene delivery to cells and tissue. Adv Drug Deliver Rev 55, 329-347, doi:https://doi.org/10.1016/S0169-409X(02)00228-4 (2003).

347 Farokhzad, O. C. \& Langer, R. Impact of Nanotechnology on Drug Delivery. Acs Nano 3, 16-20, doi:10.1021/nn900002m (2009).

348 Olmsted, S. S. et al. Diffusion of macromolecules and virus-like particles in human cervical mucus. Biophys J 81, 1930-1937, doi:10.1016/S0006-3495(01)75844-4 (2001).

349 Steinbach, J. M., Weller, C. E., Booth, C. J. \& Saltzman, W. M. Polymer nanoparticles encapsulating siRNA for treatment of HSV-2 genital infection. Journal of Controlled Release 162, 102-110, doi:https://doi.org/10.1016/j.jconrel.2012.06.008 (2012).

350 Liu, Y. \& Cao, A. in Methods in Enzymology Vol. 590 (ed Challa Vijaya Kumar) 131 (Academic Press, 2017). 
351 Sims, L. B. et al. Surface-Modified Melphalan Nanoparticles for Intravitreal Chemotherapy of RetinoblastomaMelphalan Nanoparticles for Retinoblastoma. Investigative Ophthalmology \& Visual Science 60, 1696-1705, doi:10.1167/iovs.18-26251 (2019).

352 Sims, L. B. et al. Efficacy of Surface-Modified PLGA Nanoparticles as a Function of Cervical Cancer Type. Pharmaceutical Research 36, 66, doi:10.1007/s11095019-2602-y (2019).

353 Sims, L. B., Curtis, L. T., Frieboes, H. B. \& Steinbach-Rankins, J. M. Enhanced uptake and transport of PLGA-modified nanoparticles in cervical cancer. $J$ Nanobiotechnology 14, 33, doi:10.1186/s12951-016-0185-x (2016).

354 Sims, L. B., Huss, M. K., Frieboes, H. B. \& Steinbach-Rankins, J. M. Distribution of PLGA-modified nanoparticles in 3D cell culture models of hypo-vascularized tumor tissue. Journal of Nanobiotechnology 15, 67, doi:10.1186/s12951-0170298-x (2017).

355 Martin, D. T. et al. Surface-modified nanoparticles enhance transurothelial penetration and delivery of survivin siRNA in treating bladder cancer. Mol Cancer Ther 13, 71-81, doi:10.1158/1535-7163.MCT-13-0502 (2014).

356 Xing, Z. et al. IL-6 is an antiinflammatory cytokine required for controlling local or systemic acute inflammatory responses. The Journal of Clinical Investigation 101, 311-320, doi:10.1172/JCl1368 (1998).

357 Tyo, K. M. et al. Relating Advanced Electrospun Fiber Architectures to the Temporal Release of Active Agents to Meet the Needs of Next-Generation Intravaginal Delivery Applications. Pharmaceutics 11, 160 (2019).

358 Yoon, H. \& Kim, G. A three-dimensional polycaprolactone scaffold combined with a drug delivery system consisting of electrospun nanofibers. J Pharm Sci-Us 100, 424-430, doi:10.1002/jps.22310 (2011).

359 Lusvarghi, S. \& Bewley, C. A. Griffithsin: An Antiviral Lectin with Outstanding Therapeutic Potential. Viruses 8, doi:10.3390/v8100296 (2016).

360 Fuqua, J. L., Hamorsky, K., Khalsa, G., Matoba, N. \& Palmer, K. E. Bulk production of the antiviral lectin griffithsin. Plant Biotechnology Journal 13, 1160-1168, doi:10.1111/pbi.12433 (2015).

361 O'keefe, B. R. et al. (Google Patents, 2018).

362 Yoo, H. S., Kim, T. G. \& Park, T. G. Surface-functionalized electrospun nanofibers for tissue engineering and drug delivery. Adv Drug Deliver Rev 61, 1033-1042, doi:https://doi.org/10.1016/j.addr.2009.07.007 (2009).

363 Braghirolli, D. I., Steffens, D. \& Pranke, P. Electrospinning for regenerative medicine: a review of the main topics. Drug Discovery Today 19, 743-753, doi:https://doi.org/10.1016/j.drudis.2014.03.024 (2014).

364 Masson, L. et al. Genital inflammation and the risk of HIV acquisition in women. Clin Infect Dis 61, 260-269, doi:10.1093/cid/civ298 (2015).

365 Paudel, V. \& Baral, K. P. Women living with HIV/AIDS (WLHA), battling stigma, discrimination and denial and the role of support groups as a coping strategy: a review of literature. Reprod Health 12, 53-53, doi:10.1186/s12978-015-0032-9 (2015).

366 Dobard, C. et al. Durable protection from vaginal simian-human immunodeficiency virus infection in macaques by tenofovir gel and its relationship to drug levels in tissue. Journal of virology 86, 718-725, doi:10.1128/JVI.05842-11 (2012).

367 Perry, C. M. \& Faulds, D. Valaciclovir. Drugs 52, 754-772, doi:10.2165/00003495199652050-00009 (1996). 
368 Crumpacker, C. S. Mechanism of action of foscarnet against viral polymerases. The American Journal of Medicine 92, S3-S7, doi:https://doi.org/10.1016/00029343(92)90329-A (1992).

369 Emery, S. \& Winston, A. Raltegravir: a new choice in HIV and new chances for research. The Lancet 374, 764-766, doi:https://doi.org/10.1016/S01406736(09)61392-1 (2009).

370 Dey, B. et al. Multiple antiviral activities of cyanovirin-N: blocking of human immunodeficiency virus type 1 gp120 interaction with CD4 and coreceptor and inhibition of diverse enveloped viruses. Journal of virology 74, 4562-4569, doi:10.1128/jvi.74.10.4562-4569.2000 (2000).

371 Keeffe, J. R. et al. Designed oligomers of cyanovirin-N show enhanced HIV neutralization. Proceedings of the National Academy of Sciences of the United States of America 108, 14079-14084, doi:10.1073/pnas.1108777108 (2011).

372 Mazalovska, M. \& Kouokam, J. C. Lectins as Promising Therapeutics for the Prevention and Treatment of HIV and Other Potential Coinfections. BioMed Research International 2018, 12, doi:10.1155/2018/3750646 (2018).

373 Tetteh, R. A. et al. Pre-Exposure Prophylaxis for HIV Prevention: Safety Concerns. Drug Saf 40, 273-283, doi:10.1007/s40264-017-0505-6 (2017).

374 Flash, C. A., Dale, S. K. \& Krakower, D. S. Pre-exposure prophylaxis for HIV prevention in women: current perspectives. Int $J$ Womens Health 9, 391-401, doi:10.2147/IJWH.S113675 (2017).

375 Van der Elst, E. M. et al. High Acceptability of HIV Pre-exposure Prophylaxis but Challenges in Adherence and Use: Qualitative Insights from a Phase I Trial of Intermittent and Daily PrEP in At-Risk Populations in Kenya. AIDS and Behavior 17, 2162-2172, doi:10.1007/s10461-012-0317-8 (2013).

376 Huang, Z. M., Zhang, Y. Z., Kotaki, M. \& Ramakrishna, S. A review on polymer nanofibers by electrospinning and their applications in nanocomposites. Compos Sci Technol 63, 2223-2253, doi:10.1016/S0266-3538(03)00178-7 (2003).

377 Jiang, T., Carbone, E. J., Lo, K. W. H. \& Laurencin, C. T. Electrospinning of polymer nanofibers for tissue regeneration. Prog Polym Sci 46, 1-24, doi:10.1016/j.progpolymsci.2014.12.001 (2015).

378 Yohe, S. T., Colson, Y. L. \& Grinstaff, M. W. Superhydrophobic materials for tunable drug release: using displacement of air to control delivery rates. $\mathrm{J} \mathrm{Am}$ Chem Soc 134, 2016-2019, doi:10.1021/ja211148a (2012).

379 Li, C., Vepari, C., Jin, H. J., Kim, H. J. \& Kaplan, D. L. Electrospun silk-BMP-2 scaffolds for bone tissue engineering. Biomaterials 27, 3115-3124, doi:10.1016/j.biomaterials.2006.01.022 (2006).

380 Cai, S., Xu, H., Jiang, Q. \& Yang, Y. Novel 3D electrospun scaffolds with fibers oriented randomly and evenly in three dimensions to closely mimic the unique architectures of extracellular matrices in soft tissues: fabrication and mechanism study. Langmuir 29, 2311-2318, doi:10.1021/la304414j (2013).

$381 \mathrm{Li}, \mathrm{M}$. et al. Electrospun protein fibers as matrices for tissue engineering. Biomaterials 26, 5999-6008, doi:10.1016/j.biomaterials.2005.03.030 (2005).

382 Zahedi, P., Rezaeian, I., Ranaei-Siadat, S. O., Jafari, S. H. \& Supaphol, P. A review on wound dressings with an emphasis on electrospun nanofibrous polymeric bandages. Polymers for Advanced Technologies 21, 77-95, doi:10.1002/pat.1625 (2010).

383 Vaidya, P., Grove, T., Edgar, K. J. \& Goldstein, A. S. Surface grafting of chitosan shell, polycaprolactone core fiber meshes to confer bioactivity. J Bioact Compat Pol 30, 258-274, doi:10.1177/0883911515571147 (2015). 
384 Rim, N. G. et al. Mussel-inspired surface modification of poly(L-lactide) electrospun fibers for modulation of osteogenic differentiation of human mesenchymal stem cells. Colloids Surf B Biointerfaces 91, 189-197, doi:10.1016/j.colsurfb.2011.10.057 (2012).

385 Yao, C., Li, X., Neoh, K. G., Shi, Z. \& Kang, E. T. Surface modification and antibacterial activity of electrospun polyurethane fibrous membranes with quaternary ammonium moieties. Journal of Membrane Science 320, 259-267, doi:https://doi.org/10.1016/j.memsci.2008.04.012 (2008).

386 Kangwansupamonkon, W., Tiewtrakoonwat, W., Supaphol, P. \& Kiatkamjornwong, $\mathrm{S}$. Surface modification of electrospun chitosan nanofibrous mats for antibacterial activity. Journal of Applied Polymer Science 131, doi:10.1002/app.40981 (2014).

387 Moulaei, T. et al. Monomerization of viral entry inhibitor griffithsin elucidates the relationship between multivalent binding to carbohydrates and anti-HIV activity. Structure 18, 1104-1115, doi:10.1016/j.str.2010.05.016 (2010).

388 Barton, C. et al. Activity of and effect of subcutaneous treatment with the broadspectrum antiviral lectin griffithsin in two laboratory rodent models. Antimicrob Agents Chemother 58, 120-127, doi:10.1128/AAC.01407-13 (2014).

389 Ziolkowska, N. E. et al. Domain-swapped structure of the potent antiviral protein griffithsin and its mode of carbohydrate binding. Structure 14, 1127-1135, doi:10.1016/j.str.2006.05.017 (2006).

390 Ziolkowska, N. E. et al. Crystallographic, thermodynamic, and molecular modeling studies of the mode of binding of oligosaccharides to the potent antiviral protein griffithsin. Proteins 67, 661-670, doi:10.1002/prot.21336 (2007).

391 Lai, S. K., Wang, Y. Y. \& Hanes, J. Mucus-penetrating nanoparticles for drug and gene delivery to mucosal tissues. Adv Drug Deliv Rev 61, 158-171, doi:10.1016/j.addr.2008.11.002 (2009).

392 Lai, S. K., Wang, Y. Y., Hida, K., Cone, R. \& Hanes, J. Nanoparticles reveal that human cervicovaginal mucus is riddled with pores larger than viruses. Proc Natl Acad Sci U S A 107, 598-603, doi:10.1073/pnas.0911748107 (2010).

393 Lai, S. K., Wang, Y. Y., Wirtz, D. \& Hanes, J. Micro- and macrorheology of mucus. Adv Drug Deliver Rev 61, 86-100, doi:10.1016/j.addr.2008.09.012 (2009).

394 Gentile, P., Chiono, V., Carmagnola, I. \& Hatton, P. V. An overview of poly(lacticco-glycolic) acid (PLGA)-based biomaterials for bone tissue engineering. Int $\mathrm{J} \mathrm{Mol}$ Sci 15, 3640-3659, doi:10.3390/ijms15033640 (2014).

395 Barton, C., Kouokam, J. C., Hurst, H. \& Palmer, K. E. Pharmacokinetics of the Antiviral Lectin Griffithsin Administered by Different Routes Indicates Multiple Potential Uses. Viruses 8, doi:10.3390/v8120331 (2016).

396 Sawicka, K., Gouma, P. \& Simon, S. Electrospun biocomposite nanofibers for urea biosensing. Sensor Actuat B-Chem 108, 585-588, doi:10.1016/j.snb.2004.12.013 (2005).

397 Liu, X. et al. Electrospinnability of Poly Lactic-co-glycolic Acid (PLGA): the Role of Solvent Type and Solvent Composition. Pharmaceutical Research 34, 738-749, doi:10.1007/s11095-017-2100-z (2017).

398 Bhardwaj, N. \& Kundu, S. C. Electrospinning: a fascinating fiber fabrication technique. Biotechnol Adv 28, 325-347, doi:10.1016/j.biotechadv.2010.01.004 (2010).

399 Fong, H., Chun, I. \& Reneker, D. H. Beaded nanofibers formed during electrospinning. Polymer 40, 4585-4592, doi:https://doi.org/10.1016/S00323861(99)00068-3 (1999). 
400 Rodoplu, D. \& Mutlu, M. Effects of Electrospinning Setup and Process Parameters on Nanofiber Morphology Intended for the Modification of Quartz Crystal Microbalance Surfaces. J Eng Fiber Fabr 7, 118-123 (2012).

401 Grabarek, Z. \& Gergely, J. Zero-Length Crosslinking Procedure with the Use of Active Esters. Anal Biochem 185, 131-135, doi:Doi 10.1016/0003-2697(90)90267D (1990).

402 Staros, J. V., Wright, R. W. \& Swingle, D. M. Enhancement by NHydroxysulfosuccinimide of Water-Soluble Carbodiimide-Mediated Coupling Reactions. Anal Biochem 156, 220-222, doi:Doi 10.1016/0003-2697(86)90176-4 (1986).

403 Tan, S. H., Inai, R., Kotaki, M. \& Ramakrishna, S. Systematic parameter study for ultra-fine fiber fabrication via electrospinning process. Polymer 46, 6128-6134, doi:10.1016/j.polymer.2005.05.068 (2005).

404 Spasova, M., Stoilova, O., Manolova, N., Rashkov, I. \& Altankov, G. Preparation of PLLA/PEG Nanofibers by Electrospinning and Potential Applications. J Bioact Compat Pol 22, 62-76, doi:10.1177/0883911506073570 (2007).

405 Boland, E. D. et al. Electrospinning polydioxanone for biomedical applications. Acta Biomater 1, 115-123, doi:10.1016/j.actbio.2004.09.003 (2005).

406 Senecal, A., Magnone, J., Marek, P. \& Senecal, K. Development of functional nanofibrous membrane assemblies towards biological sensing. React Funct Polym 68, 1429-1434, doi:10.1016/j.reactfunctpolym.2008.06.022 (2008).

407 Zhang, Y. Z., Venugopal, J., Huang, Z. M., Lim, C. T. \& Ramakrishna, S. Characterization of the surface biocompatibility of the electrospun PCL-collagen nanofibers using fibroblasts. Biomacromolecules 6, 2583-2589, doi:10.1021/bm050314k (2005).

408 Gupta, D., Venugopal, J., Mitra, S., Giri Dev, V. R. \& Ramakrishna, S. Nanostructured biocomposite substrates by electrospinning and electrospraying for the mineralization of osteoblasts. Biomaterials 30, 2085-2094, doi:10.1016/j.biomaterials.2008.12.079 (2009).

409 O'Keefe, B. R. et al. Scaleable manufacture of HIV-1 entry inhibitor griffithsin and validation of its safety and efficacy as a topical microbicide component. Proc Natl Acad Sci U S A 106, 6099-6104, doi:10.1073/pnas.0901506106 (2009).

410 Akkouh, O. et al. Lectins with anti-HIV activity: a review. Molecules 20, 648-668, doi:10.3390/molecules20010648 (2015). 


\section{APPENDICES \\ FABRICATION AND CHARACTERIZATION OF GRIFFITHSIN-MODIFIED FIBER SCAFFOLDS FOR PREVENTION OF SEXUALLY TRANSMITTED INFECTIONS}

\section{Introduction}

During the past two decades, EFs have been extensively used in the fields of drug delivery and tissue engineering ${ }^{108}$. Often, biocompatible polymers are selected to easily translate to therapeutic applications. To fabricate polymeric EFs, the selected polymer is dissolved in an organic solvent or aqueous solution, depending on the degree of polymer hydrophobicity ${ }^{377}$. Active agents of interest are then added to the solvent or aqueous solution prior to the electrospinning process. The polymer solution is then aspirated into a syringe and slowly ejected while subject to an electrical current. This process typically results in polymer fibers with sheet or cylindrical macrostructures (Figure A.1), and fiber diameters ranging from the micro- to nano-scale ${ }^{80}$. For most therapeutic applications, active agents are incorporated within the fibers during the electrospinning process and are released from the fiber via diffusion and subsequent fiber degradation. The rate of degradation or release may be altered by using different types of polymers or polymer blends to establish a desired release profile, imparting unique chemical and physical properties ${ }^{376}$, and promoting the encapsulation of virtually any 
compound. As such, EFs have proven beneficial to the delivery of small molecule drugs and biological agents including proteins, peptides, oligonucleotides, and growth factors ${ }^{80,110,183}$. 


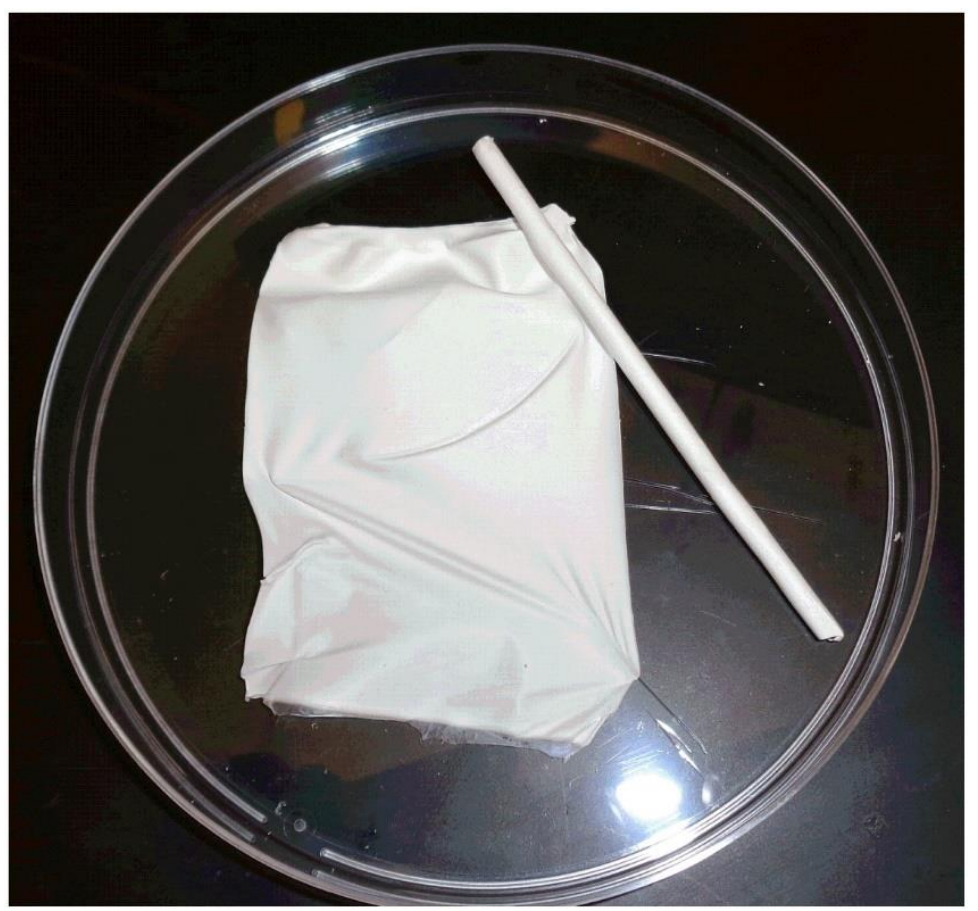

Figure A.1 The macroscale morphology of electrospun fibers. The electrospun fibers shown were fabricated using a $4 \mathrm{~mm}$ (cylinder) and $25 \mathrm{~mm}$ (sheet) diameter mandrel, respectively. 
In the field of STI prevention, EFs have been recently used to incorporate and provide sustained- or inducible-release of antiviral agents $38,83-87,101,160,166,378$. In one of the earliest studies, $\mathrm{pH}$-responsive fibers were developed to release active agents in response to environmental changes within the female reproductive tract (FRT), as an on-demand method of protection against HIV-183. Since, other studies have investigated polymer blends comprised of polyethylene oxide (PEO) and poly-L-lactic acid (PLLA), to evaluate the tunable release of antiviral and contraceptive agents for HIV-1 prevention and contraception in vitro ${ }^{86}$. Additional studies have demonstrated the feasibility of EFs to provide the following: prolonged release of small molecule antivirals ${ }^{87}$, strong and flexible mechanical properties ${ }^{379}$, 3-D delivery architectures ${ }^{380}$, inhibition of sperm penetration ${ }^{86}$, and the ability to merge with other delivery technologies ${ }^{378}$. Finally, previous work has evaluated polymeric fibers for the sustained-delivery of antiviral agents against common coinfective viruses, HSV-2 and HIV-1 ${ }^{87}$. In this study, polymer fibers provided complementary activity to antiviral delivery by retaining their structure for up to 1 month and providing a physical barrier to viral entry. From these results, it was observed that EFs may be used to both physically and chemically hinder virus infection.

While tunable release properties make polymeric EFs an attractive delivery platform for microbicide delivery, EFs have been developed in other applications to serve as surface-modified scaffolds ${ }^{376}$. EFs have been used to mimic the morphology of the extracellular matrix (ECM), often acting as scaffolds to improve cellular regeneration ${ }^{381}$, and enhance their utility in tissue engineering ${ }^{211,382}$. Fibers 
comprised of polymers such as poly- $\varepsilon$-caprolactone (PCL) and PLLA have been surface-modified with growth factors and proteins after electrospinning to impart ECM-like properties including increased cellular adhesion and proliferation ${ }^{383,384}$. Additionally, antimicrobial surface-modified EFs have been evaluated to prevent the growth of specific pathogenic bacteria ${ }^{385,386}$. Due to this versatility and the ability to induce biological effects, EF technology continues to expand across a variety of fields to provide multi-mechanistic functionality. Yet, despite their utility in a diversity of applications, surface-modified fibers have only recently been explored in the microbicide field ${ }^{75}$.

In parallel with the development of new delivery technologies to prevent and treat STIs, novel biological therapeutics have been developed. One of the most promising microbicide candidates is the adhesive antiviral lectin, GRFT ${ }^{66}$. Originally derived from a species of red algae, GRFT has demonstrated activity as a potent inhibitor of HIV-1, HSV-2, SARS, as well as Hepatitis C virus ${ }^{62-65,67,70}$. In fact, among biologically-based inhibitors, GRFT has one of the most potent antiHIV activities, inactivating HIV-1 almost immediately upon contact ${ }^{66}$, while maintaining stability and activity in the presence of culture media from vaginal microbes for up to 10 days ${ }^{69}$. More recently, a $0.1 \%$ GRFT gel was shown to protect mice against intravaginal HSV-2 challenge, making it a promising candidate for the first line of protection against both HSV-2 and HIV-165,387. For HIV-1 specifically, GRFT inhibits infection by physically binding gp120 or terminal mannose $N$-linked glycan residues on viral envelope surfaces to prevent entry ${ }^{61,387-}$ 390. This inhibition is highly potent, with $\mathrm{IC}_{50}$ s approaching $3 \mathrm{ng} / \mathrm{mL}^{62}$. In addition to 
inhibiting HIV-1 infection, studies have also shown that GRFT protects against HSV-2 infection by inhibiting the cell-to-cell spread of the virus ${ }^{65}$. In all cases, GRFT has been shown to be adhesive to viral particles, while demonstrating high resistance to denaturation. Last, GRFT has demonstrated synergistic activity with combinations of Tenofovir (TFV) and other antivirals ${ }^{72}$, making it feasible and likely beneficial to co-administer with EFs. The potent properties of GRFT make it an excellent biologically-based antiviral candidate, in which delivery may be enhanced with EF technology.

Utilizing this knowledge of the adhesive and innate antiviral properties of GRFT, a polymeric fiber scaffold was designed, that integrates these properties to provide the first layer of virus entry inhibition ${ }^{75}$. Finding inspiration in the way that cervicovaginal mucus hinders virus transport primarily through mucoadhesive mucin interactions, we hypothesized that by using EFs as a scaffold and covalently modifying the surface with GRFT, a high density of surface-conjugated GRFT would debilitate and inactivate virus at its entry point ${ }^{391-393}$. Here EFs were developed as a stationary scaffold to provide a protein-based, viral adhesiveinactivating barrier platform. We sought to combine the potent antiviral properties of GRFT with a biocompatible, modifiable, and durable polymer platform, to create a novel virus "trap."

To achieve these goals, fibers comprised of PLGA were electrospun, and EDC-NHS chemistry was used to subsequently modify the EF surface with GRFT. PLGA served as a model polymer due to its extensive use in electrospinning ${ }^{394}$, combined with its biocompatibility and cost-effectiveness. Additionally, surface 
modification exploits the large surface area of EFs, and provides a useful alternative that can be combined with encapsulation to maximize fiber utility ${ }^{88}$. Unlike traditional encapsulation methods where only a portion of GRFT is available (and only transiently present in the FRT), surface modification may enable GRFT to maintain maximum bioactivity during the entire duration of treatment. Furthermore, the incorporation of hydrophilic compounds such as proteins, by traditional electrospinning methods, may result in lower encapsulation efficiencies and loss of protein activity ${ }^{362}$. Therefore, GRFT surface-modified fibers may offer a promising alternative delivery method that can be used alone or in combination with electrospinning to enhance protection against STI infection.

\section{Protocol}

\section{Preparation and Fabrication of the Electrospun Fiber Scaffold}

CAUTION: All work with solvents or polymer solutions should be performed in a chemical fume hood. Refer to material safety datasheet of each reagent before starting the protocol.

1. To electrospin a $3 \mathrm{~mL} 15 \% \mathrm{w} / \mathrm{w}$ PLGA polymer solution, weigh $720 \mathrm{mg}$ of $50: 50$ poly(lactic-co-glycolic acid) (PLGA; 0.55 to $0.75 \mathrm{dL} / \mathrm{g}, 31-57 \mathrm{kDa}$ ) into a $10 \mathrm{~mL}$ scintillation vial. The volume of the solution is based on the typical batch size used in current studies.

NOTE: The polymer mass to add to a given volume of solvent must be calculated by first determining the density of the solvent used to dissolve the polymer. The density of the solvent Hexafluoro-2-propanol (HFIP) is $1.59 \mathrm{~g} / \mathrm{mL}$. Thus, the weight 
of solvent, based on a volume of $3.0 \mathrm{~mL}$ HFIP needed, is $4.8 \mathrm{~g}(3.0 \mathrm{~mL} \times 1.59$ $\mathrm{g} / \mathrm{mL}$ ). For a $15 \% \mathrm{w} / \mathrm{w}$ fraction of PLGA to HFIP, $720 \mathrm{mg}$ PLGA must be added to $3.0 \mathrm{~mL} \mathrm{HFIP}(0.15 \times 4,800 \mathrm{mg}=720 \mathrm{mg})$. The advantage of using a $\% \mathrm{w} / \mathrm{w}$ polymer/solution, rather than $\% \mathrm{w} / \mathrm{v}$, is that this provides a defined weight of the final solution. This defined weight enables more accurate solvent replacement, in the case of solvent evaporation during step 1.3.

2. Add $3.0 \mathrm{~mL}$ HFIP to the glass scintillation vial containing PLGA (from step 1.1) using a serological glass pipette. Cover the vial with plastic film, then measure and record the vial mass.

3. Incubate the polymer suspension overnight at $37^{\circ} \mathrm{C}$ to ensure complete dissolution of the polymer. If any solvent evaporates, decreasing the vial mass, add HFIP until the vial reaches its original mass in step 1.2.

4. After incubation, prepare the electrospinning apparatus (Figure A.2A). Although a mandrel of any size may be used, here a rotating $25 \mathrm{~mm}$ outer-diameter stainless steel mandrel was used as the collector.

NOTE: A larger mandrel diameter will decrease the fiber thickness, given the same volume of electrospinning solution. 

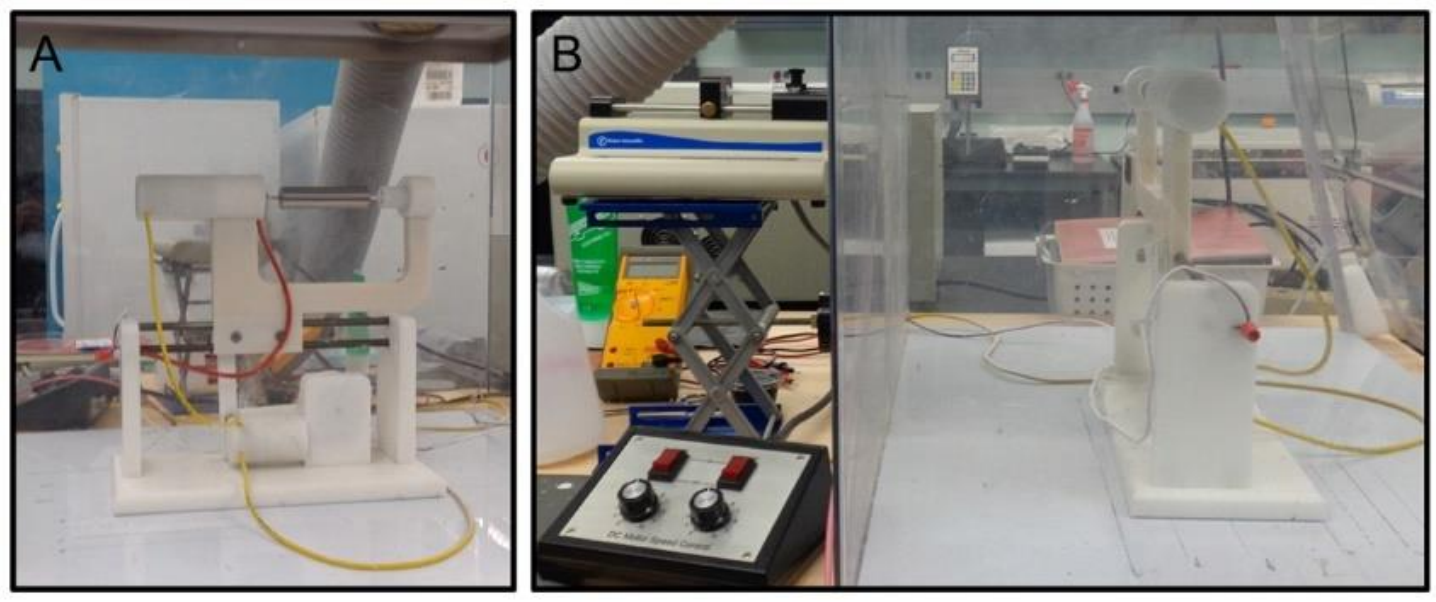

Figure A.2 Electrospinning apparatus. (A) The collecting mandrel where the liquid jets of polymer deposit, and (B) the full electrospinning setup. 
5. Aspirate the polymer solution into a $3 \mathrm{~mL}$ syringe.

6. Connect a blunt 18-gauge, $1 / 2$ inch needle tip to the syringe and dispense the excess solution (typically $0.25 \mathrm{~mL}$ ) to remove empty headspace in the needle tip.

7. Place the syringe on a syringe pump and set the instrument flow rate to 2.0 $\mathrm{mL} / \mathrm{h}$.

NOTE: This flow rate was previously optimized based on polymer viscosity for this formulation.

8. Connect the power source to the syringe needle and electrospin the polymer solution using a voltage of $+27 \mathrm{kV}$. The distance between the needle and collector should be set to approximately $25 \mathrm{~cm}$ (Figure A.2B.

CAUTION: The electrospinning process creates a solvent vapor. Use a fume hood or an enclosed apparatus (Figure A.2) to remove the harmful vapor.

9. Once the entire solution is electrospun, turn off the power source and allow the mandrel to spin for an additional 30 min to fully evaporate solvent.

10. Turn off the rotating mandrel collector, and use a razor blade to cut the fiber from the mandrel. Use the blade to gently peel the fiber from the mandrel.

11. Collect the electrospun PLGA fiber into a labeled Petri dish, and place in a desiccator overnight to remove residual solvent.

\section{Surface-modification of Fibers with GRFT}


1. Prepare solutions of phosphate-buffered saline (PBS) and 2-(N-morpholino) ethanesulfonic acid (MES buffer). Prepare PBS by dissolving $8 \mathrm{~g} \mathrm{NaCl}, 0.2 \mathrm{~g} \mathrm{KCl}$, $1.44 \mathrm{~g} \mathrm{Na}_{2} \mathrm{HPO}_{4}$, and $0.24 \mathrm{~g} \mathrm{KH}_{2} \mathrm{PO}_{4}$ in $1 \mathrm{~L}$ of ultrapure water. Similarly, dissolve 19.52 $\mathrm{g}$ MES (free acid, MW 195.2) and $29.22 \mathrm{~g} \mathrm{NaCl}$ in $1 \mathrm{~L}$ of ultrapure water, to prepare MES buffer. Ensure the final pH of each solution is between $7.2-7.5$ and 5.0 - 6.0, respectively, using a $\mathrm{pH}$ meter.

2. Prepare individual working solutions of EDC (2 mM) and NHS (5 mM). Remove the EDC and NHS from the freezer and allow them to equilibrate to room temperature before weighing. Weigh $4 \mathrm{mg}$ of EDC into a $1.5 \mathrm{~mL}$ microcentrifuge tube. Weigh $6 \mathrm{mg}$ of NHS into another microcentrifuge tube. Add $1 \mathrm{~mL}$ MES buffer to each tube. Vortex both tubes vigorously to ensure the reagents are fully dissolved.

3. Prepare a solution of hydroxylamine by weighing $70 \mathrm{mg}$ into a $50 \mathrm{~mL}$ conical centrifuge tube.

4. Add $20 \mathrm{~mL}$ PBS to the hydroxylamine and vortex to dissolve.

5. Mass out an appropriate amount of PLGA fiber into a $15 \mathrm{~mL}$ conical centrifuge tube. Typically, $75 \mathrm{mg}$ of fiber is used for each reaction batch.

6. Add $8 \mathrm{~mL}$ of MES buffer to the $15 \mathrm{~mL}$ tube.

7. Add $1 \mathrm{~mL}$ each of the EDC and NHS solutions prepared earlier to the tube. The final volume of the solution should be $10 \mathrm{~mL}$. The final concentrations of EDC and NHS should be $0.4 \mathrm{mg} / \mathrm{mL}$ and $0.6 \mathrm{mg} / \mathrm{mL}$, respectively. 
8. Close and seal the $15 \mathrm{~mL}$ tube with plastic film and place on a rotator to allow the solution to be gently inverted for $15 \mathrm{~min}$ at room temperature (Figure A.3B). This step activates the carboxyl groups on the polymer to allow for covalent modification with the GRFT protein (Figure A.3A). 
A.

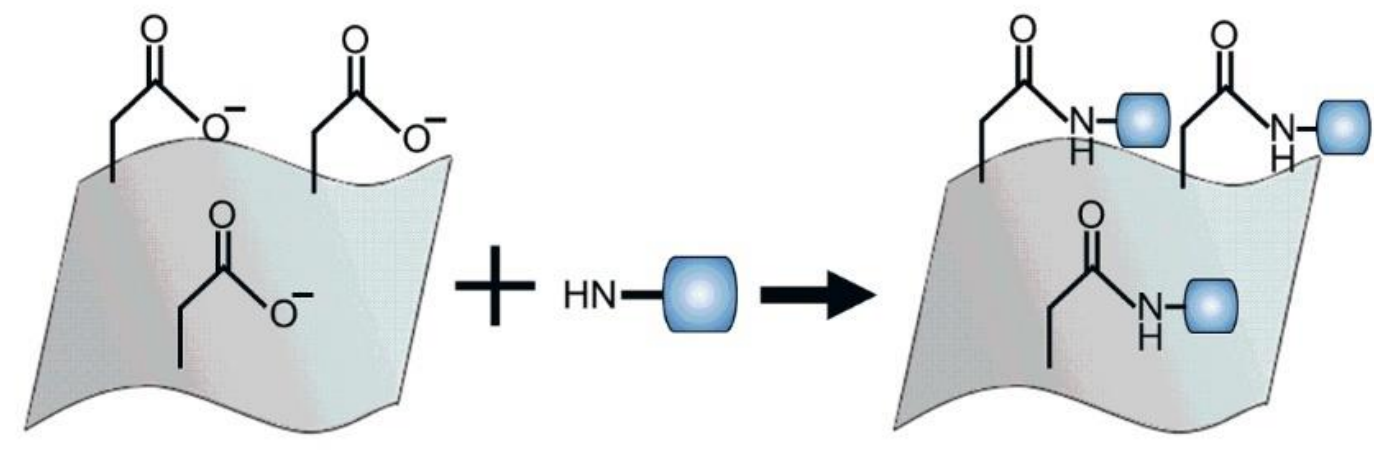

B.

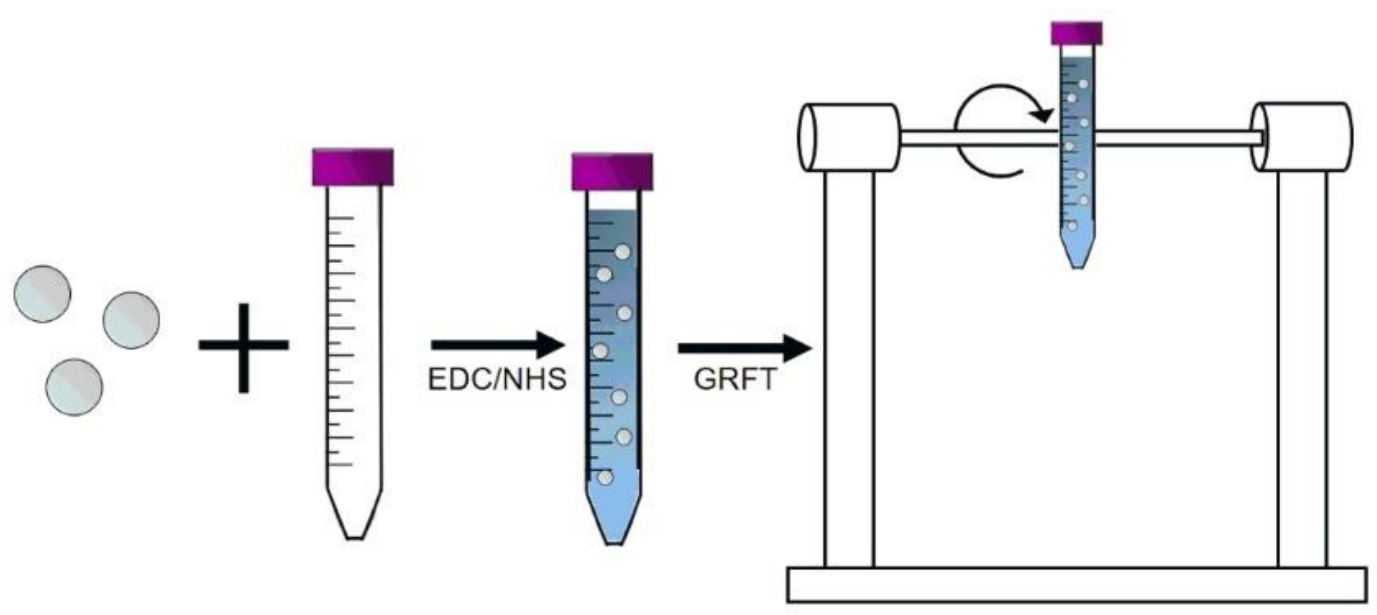

Figure A.3 Schematic of EF modification with GRFT using EDC-NHS chemistry. (A) Carboxyl groups on the PLGA EF react with NHS in the presence of EDC to form amine-reactive esters, which will subsequently form stable amide bonds with the primary amines of GRFT. (B) Two milligram fiber disks or pieces are cut and incubated in $8 \mathrm{~mL}$ of MES buffer with $2 \mathrm{~mL}$ of EDC/NHS reagent and rotated for $15 \min$. 
9. After activation, carefully quench the reaction by adding $14 \mu \mathrm{L}$ of $\beta$ mercaptoethanol to the tube. Invert the tube several times to ensure complete mixing.

CAUTION: $\beta$-mercaptoethanol is highly toxic and should only be used in a chemical fume hood.

10. Discard the supernatant and rinse the PLGA fiber twice, with $10 \mathrm{~mL}$ of PBS, to remove any remaining $\beta$-mercaptoethanol.

11. After rinsing, add an appropriate amount of GRFT stock solution to the tube. For example, a $5 \mathrm{nmol}$ GRFT/mg fiber would require $6.35 \mu \mathrm{L}$ of GRFT stock solution (from a $10 \mathrm{mg} / \mathrm{mL}$ stock) per $\mathrm{mg}$ of fiber. Thus a $75 \mathrm{mg}$ fiber sample would require $476.25 \mu \mathrm{L}$ of $10 \mathrm{mg} / \mathrm{mL}$ GRFT stock solution.

NOTE: GRFT fibers with theoretical loadings of $0.05,0.5$, and 5 nmol GRFT per mg fiber were fabricated.

12. Add enough PBS to bring the final volume to $8 \mathrm{~mL}$, close and invert the tube to ensure thorough mixing.

13. Seal the tube with plastic film and place on a rotor again, this time for $2 \mathrm{~h}$.

14. After the $2 \mathrm{~h}$ incubation, quench the reaction by adding $2 \mathrm{~mL}$ of hydroxylamine solution into the $15 \mathrm{~mL}$ centrifuge tube. Per manufacturer instructions, the final concentration of hydroxylamine during the quenching reaction should be 0.7 $\mathrm{mg} / \mathrm{mL}$. 
15. Mix the solution well and discard the supernatant. Rinse the surface-modified PLGA fiber twice with $10 \mathrm{~mL}$ ultrapure water to remove any unconjugated GRFT.

16. Transfer the fiber to a Petri dish and place inside of a desiccator until the fiber is completely dry. Transfer the Petri dish to $4{ }^{\circ} \mathrm{C}$ for storage.

\section{SEM Characterization of GRFT Surface-modified Fibers}

1. Place a strip of double-sided carbon tape on an SEM specimen mount. Label the bottom of the specimen mount with the sample identifying information using a permanent marker.

2. Cut three samples from one surface-modified fiber and place them on separate specimen mounts. The thickness of each sample is approximately $0.5 \mathrm{~mm}$.

3. Sputter coat the samples using electron-induced particle deposition from a gold plate. Sputter coat for $90 \mathrm{~s}$, at $2.4 \mathrm{kV}$.

NOTE: The sputter coat time may vary depending on equipment parameters, including voltage and amperage.

4. Image the samples at $8 \mathrm{kV}$ with a magnification ranging from 1,000 to $5,000 \mathrm{X}$.

\section{Extraction of GRFT from Surface-modified Fibers}

1. Mass out $2 \mathrm{mg}$ of fiber in triplicate into $1.5 \mathrm{~mL}$ microcentrifuge tubes.

2. Add $1 \mathrm{~mL}$ dimethyl sulfoxide (DMSO) to the tube, then vortex and incubate for $1 \mathrm{~min}$ at room temperature to completely dissolve the fiber. 
3. After incubation, dilute a $10 \mu \mathrm{L}$ aliquot of the DMSO-fiber solution from step 4.2, at least 100 -fold in Tris-EDTA (TE) buffer $(\mathrm{pH}=8.0)$.

4. Store samples at $-20^{\circ} \mathrm{C}$ until loading characterization with ELISA.

\section{Measuring GRFT Desorption from Fibers}

1. To assess the amount of GRFT released or desorbed from the fiber, weigh 5 $10 \mathrm{mg}$ of surface-modified fiber and place in a microcentrifuge tube. Record the fiber mass in each tube.

2. Add $1 \mathrm{~mL}$ of an appropriate solution that mimics the physiological environment (e.g., PBS, TE buffer, simulated vaginal fluid (SVF), etc.) to each sample.

3. Incubate the samples for $1 \mathrm{~h}$ on a rotating shaker at $200 \mathrm{rpm}, 37^{\circ} \mathrm{C}$.

4. After incubation, remove approximately $1 \mathrm{~mL}$ of $\mathrm{TE}$ buffer containing the desorbed GRFT from the vial, and aliquot to cluster tubes. Store at $-20^{\circ} \mathrm{C}$ until protein quantification.

5. Transfer the sample to a new microcentrifuge tube, add $1 \mathrm{~mL}$ of fresh buffer solution to the fiber within the microcentrifuge tube, and incubate until the next time point.

6. Typical time points used to measure release include: $1,2,4,6,8,24,48,72 \mathrm{~h}$, and 1 wk. Negligible desorption was observed in these studies after $4 \mathrm{~h}$.

\section{Quantification of GRFT Extraction and Desorption via ELISA}

1. Coat a 96 -well ELISA plate with $0.1 \mathrm{~mL}$ of $\mathrm{HA}(10 \mu \mathrm{g} / \mathrm{mL})$ per well as previously 
described ${ }^{395}$. Seal the plate with plastic film and incubate overnight at $4{ }^{\circ} \mathrm{C}$ (Figure A.4). 

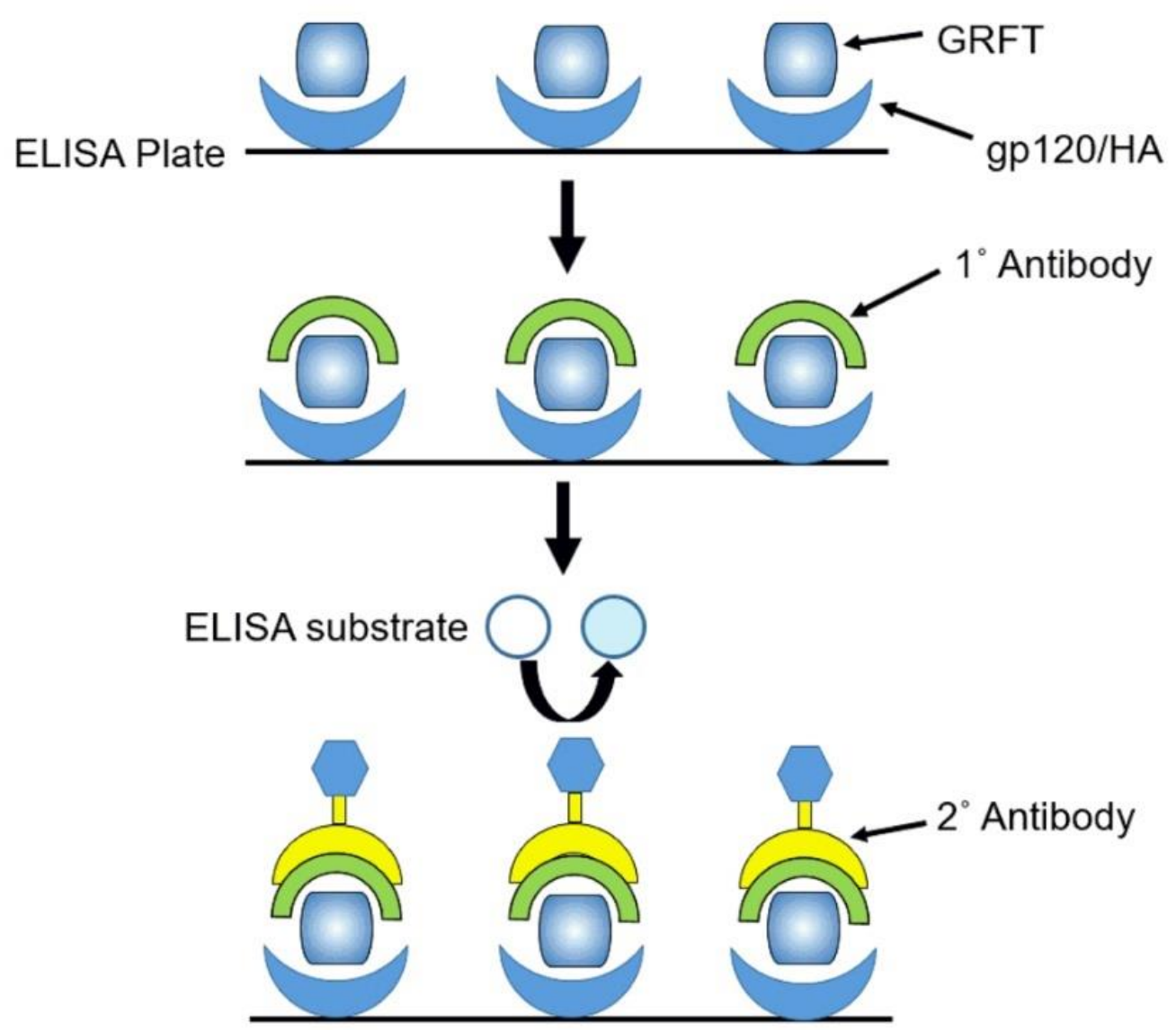

Figure A.4 Schematic illustrating GRFT quantification using ELISA. A 96-well immunoplate is coated with gp120 or HA to capture and immobilize GRFT. Primary antibodies against GRFT, secondary antibodies linked to horseradish peroxidase, and ELISA substrate are sequentially added to quantify GRFT. 
2. Remove the coating buffer, and add $0.3 \mathrm{~mL}$ blocking buffer ( 2 - $3 \%$ bovine serum albumin (BSA) in PBS with $0.05 \%$ polysorbate 20 ) to each well. Incubate the plate for at least $2 \mathrm{~h}$ at room temperature.

3. After incubation, rinse the plate 3 times with $1 \times$ PBS with $0.1 \%$ polysorbate 20 (PBS-P). After rinsing, dispense $0.1 \mathrm{~mL}$ of sample, standard, or PBS as negative control into the respective wells. Incubate the plate again for $1 \mathrm{~h}$ at room temperature.

4. Rinse the plate 3 times again with PBS-P. After rinsing, add $0.1 \mathrm{~mL}$ of primary antibody (goat anti-GRFT antiserum) into each well and incubate for at least $1 \mathrm{~h}$ at room temperature. Typically, the primary antibody solution is diluted by $1: 10,000$ in PBS.

5. After incubating the samples with primary antibody rinse the plates again 3 times with PBS-P. Add $0.1 \mathrm{~mL}$ of secondary antibody (horseradish peroxidase (HRP)conjugated rabbit anti-goat $\lg G$ ) to each well and incubate for $1 \mathrm{~h}$ at room temperature. The secondary antibody solution is diluted by 1:10,000 in PBS.

6. Wash the plate 3 times. Add $0.1 \mathrm{~mL}$ TMB 2-peroxidase substrate to each well. Monitor color development (approximately $2 \mathrm{~min}$ ), then add $0.1 \mathrm{~mL} \mathrm{H} 2 \mathrm{SO} 4(1 \mathrm{~N})$ to quench the reaction. Read plate at $450 \mathrm{~nm}$ on a plate reader.

7. Average the background OD values (wells which only receive PBS), and subtract this from the experimental groups. 


\section{Representative Results}

Fiber morphology has a significant effect on the ability of surface-modified EFs to provide protection against viruses. Although electrospinning is a convenient and straightforward procedure, non-optimized polymer formulations may result in irregular fiber morphology (Figure A.5). Alterations in electrospinning conditions that result in the formation of beaded or amorphous mat-like morphologies, are often caused by solvent polymer incompatibility, low polymer viscosity, flow rate, or other electrospinning conditions. The resulting variations in fiber structure can result in different release profiles for drug-incorporated fibers or inconsistencies in conjugation efficacy, altering the fibers ability to physically or chemically hinder virus penetration. The PLGA EFs fabricated using the described electrospinning conditions should result in distinct fiber morphologies with diameters ranging between 1.5 and $2.8 \mu \mathrm{m}$ (Figure 3.6). To determine fiber morphology and diameter, EFs should be examined with SEM prior to other characterization or modification steps. 

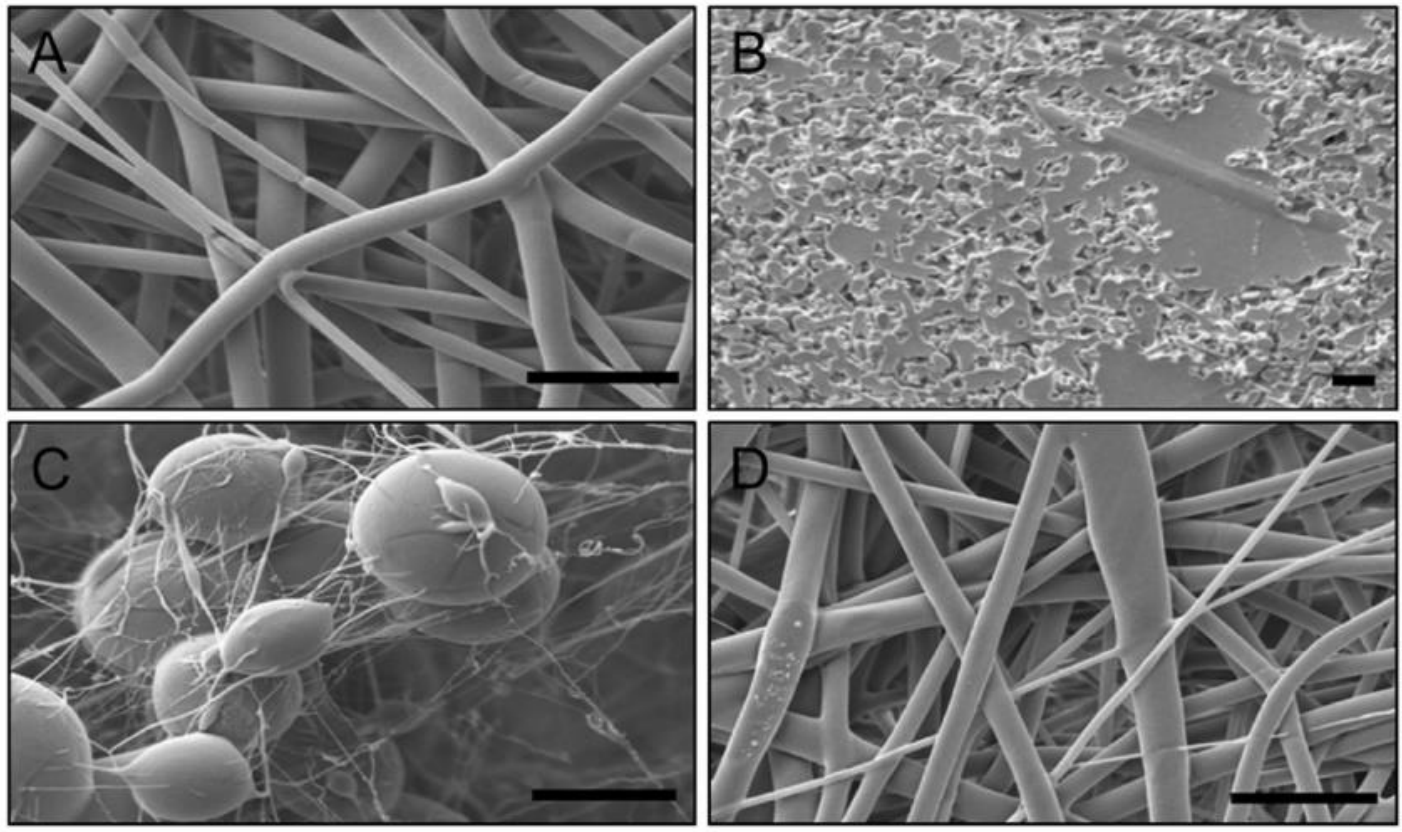

Figure A.5 Effects of solvent choice on PLGA EF morphology. (A) The 15\% w/w PLGA EFs in HFIP displayed desirable thread-like morphology. (B) The $15 \% \mathrm{w} / \mathrm{w}$ PLGA EFs in chloroform and dimethylformamide, failed to form due to non-optimal solvent choice or polymer concentration (viscosity). (C) The 15\% w/w and (D) $20 \%$ w/w PLGA EFs in TFE demonstrate the importance of polymer viscosity. Beads formed in the formulation with lower polymer concentration (C), while increasing the polymer concentration (solvent viscosity) resulted in well-defined fiber morphology (D). Note, Figure 3.5B was taken at a lower magnification to show the mat-like morphology. Scale bars $=10 \mu \mathrm{m}$. 

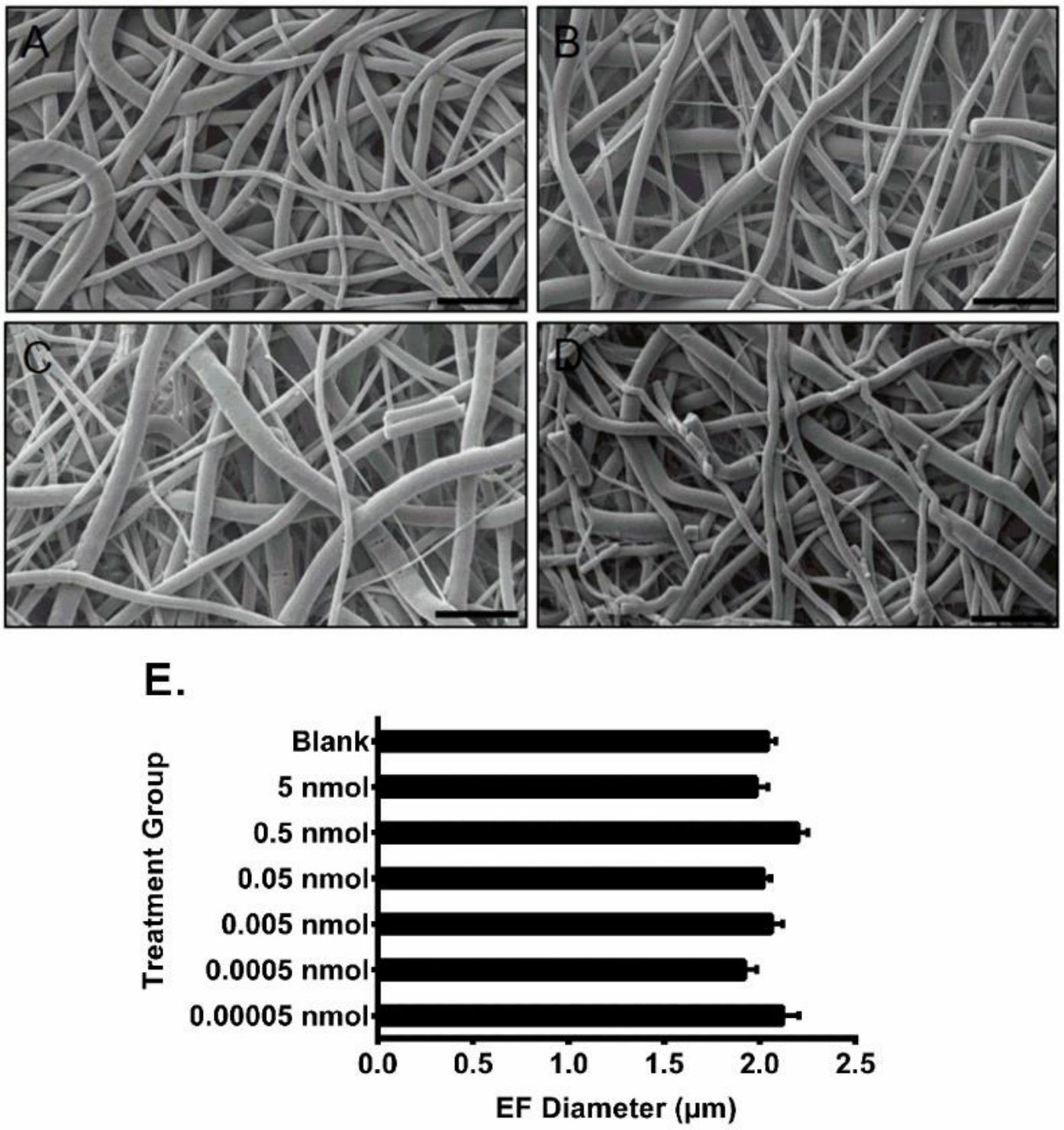

Figure A.6 SEM images and fiber diameters of bare and GRFT-modified EFs. (A) Bare PLGA EFs and PLGA EFs surface-modified with (B) $0.05 \mathrm{nmol},(C) 0.5 \mathrm{nmol}$, and (D) $5 \mathrm{nmol}$ of GRFT per mg of fiber. Scale bars $=10 \mu \mathrm{m}$. (E) Diameters of unmodified and GRFT-EFs. Error bars represent the mean \pm SEM. No statistical difference was observed between the diameters of unmodified and GRFT-modified fibers. Figure A.6E has been adapted from Grooms et al. ${ }^{75}$ 
SEM images of blank, $0.05,0.5$, and 5 nmol GRFT fibers showed no significant differences in fiber morphology (Figure A.6A-D), indicating that GRFT modification has no effect on fiber morphology. To determine the average diameter of each EF formulation, a minimum of 50 random measurements were taken per field of view from the SEM images diameters of the EF formulations were measured and calculated in ImageJ, as shown in Figure A.6E. All EF formulations had similar average diameters around $1.9 \mu \mathrm{m}$, demonstrating the consistency of unmodified fiber fabrication process across batches.

To determine the amount of GRFT conjugated to the EFs, GRFT-EFs were dissolved in DMSO, followed by a 100-fold dilution in TE buffer, to extract GRFT from the fiber. The quantity of GRFT conjugated per mg of fiber was quantified using ELISA. For each modification density $(0.05,0.5$, and $5 \mathrm{nmol}$ GRFT per $\mathrm{mg}$ fiber), ten replicates were evaluated. For the 5, 0.5, and $0.05 \mathrm{nmol}$ GRFT/mg EF modifications, each EF had 373, 165, and 42 ng GRFT per mg of EF, resulting in conjugation efficiencies of $0.6,4.2$, and $6.9 \%$, respectively. These results demonstrate that GRFT-EFs conjugated with higher theoretical surfacemodification density, result in more GRFT conjugated to the fiber (Figure 3.7A). However, there was an inverse correlation with the resulting conjugation efficiency $^{75}$ 


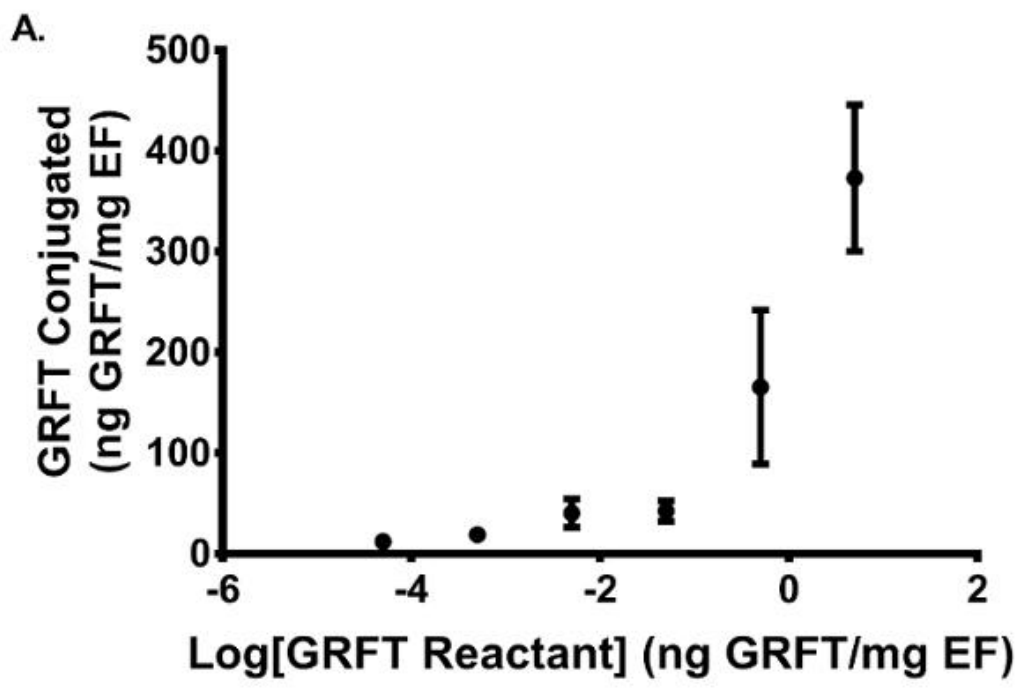

B.

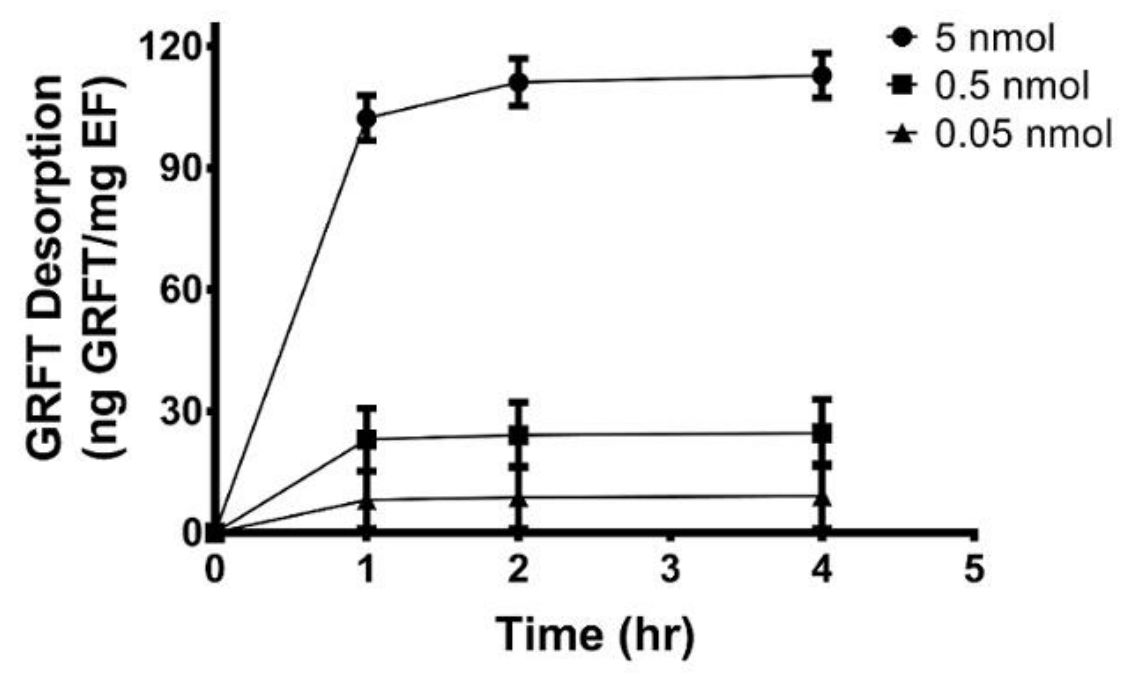

Figure A.7 Quantity of GRFT conjugated to and desorbed from GRFT-modified EF. (A) The quantity of GRFT conjugated to each mg of EF fiber increases with increased GRFT reactant concentration. (B) The quantity of GRFT released from each $\mathrm{mg}$ of fiber is shown for the $0.05,0.5$, and $5 \mathrm{nmol}$ formulations after 1,2 , and $4 \mathrm{~h}$ incubation in SVF. Error bars represent the mean \pm SEM. This figure has been adapted from Grooms et al75. 
To assess the amount of GRFT covalently conjugated to the fiber surface relative to that adsorbed, GRFT-EFs were incubated in SVF to determine the amount of GRFT released. Within the first $4 \mathrm{~h}, 113,25$, and $10 \mathrm{ng}$ of GRFT per mg EF was detected in SVF for the $5,0.5$, and $0.05 \mathrm{nmol}$ theoretical modification concentrations, respectively. These values correspond to $30 \%, 41 \%$, and $24 \%$ of the amount of GRFT conjugated to 5, 0.5, and 0.05 nmol GRFT-EFs. After $4 \mathrm{~h}$, negligible GRFT was detected in the release eluate for all three formulations. GRFT release after 1, 2, and $4 \mathrm{~h}$ is shown in Figure 3.7B. Taken together, these data indicate that the majority of GRFT is covalently bound to the EFs, and that the surface-adsorbed GRFT is released within the first $4 \mathrm{~h}$.

\section{Discussion}

Due to their porous structures and large surface areas, EFs have found a variety of applications in healthcare, one of which includes serving as therapeutic delivery vehicles. Drugs and other active agents can be incorporated within EFs for tunable delivery, while biologics and chemical ligands can be conjugated to the fiber surface for cell-specific targeting ${ }^{396}$ or biosensing ${ }^{102}$. Here the fabrication of GRFT surface-modified PLGA EFs, as a delivery scaffold to prevent HIV-1 infection, is described. GRFT-EFs were synthesized by electrospinning, providing the advantages of low cost and high production rate relative to other fiber production methods 88,102 .

\section{Critical Steps in the Protocol}


The formation of EFs is critically dependent on the properties of polymer solution, in particular the solution or solvent viscosity ${ }^{397}$. The factors that affect the viscosity of a polymer solution include polymer molecular weight, polymer concentration, and the type of solvent used. The solution or solvent viscosity is typically adjusted by changing the ratio of polymer to solvent, to obtain the desired polymer concentration. With each fabrication, the volume must be maintained during overnight incubation to maintain the proper polymer-to-solvent ratio (viscosity). At sufficiently high polymer concentration, the polymer molecules entangle in the solution during the electrospinning process to produce fibers. During the electrospinning process, a bead will form at the spinneret tip and if there is sufficient polymer entanglement, liquid jets will erupt from this point at a critical voltage and accelerate in whip-like fashion toward the collecting mandrel ${ }^{398}$. Solvent evaporation will then lead to jet thinning, producing threads of fiber as they deposit on the collecting mandrel. Once synthesized, EFs should be analyzed by SEM to verify proper morphology and consistent fiber diameter. The presence of beaded EFs may be the result of low solution viscosity ${ }^{399}$, exceedingly high applied voltage ${ }^{256}$, polymer feed rate 400 , or a combination of all three factors. If this is observed, polymer concentration should be increased and the applied voltage and feed rate should be adjusted, to attain fiber-like morphology.

To conjugate proteins to the EF surface, here PLGA carboxyl groups were reacted using EDC-NHS carbodiimide crosslinking chemistry ${ }^{401}$. During the modification process, fibers are briefly incubated with EDC in the presence of NHS which results in the conversion of carboxylates into semistable, amine-reactive 
esters. During the two-step conjugation process, it is critical that the buffers used during each step have the optimal $\mathrm{pH}$, noted in the manufacturer's instructions, to ensure maximum conjugation efficiency. The half-life of NHS esters ranges from four to five hours at neutral $\mathrm{pH}$ and dramatically decreases in more basic conditions ${ }^{402}$. Thus, the first reaction should be performed in MES buffer at $\mathrm{pH} 5$ 6 and the activated fibers should then be transferred to a PBS buffer $(\mathrm{pH} 7.2-7.5)$ for subsequent and immediate reaction with GRFT. It is also important that EDC is inactivated by the addition of 2-mercaptoethanol and sufficiently rinsed from the fibers after carboxylate activation. This will help prevent protein activation by EDC and self-crosslinking during the second reaction, which may reduce conjugation efficiency.

\section{Modifications and Troubleshooting}

Although our previous work demonstrated the efficacy of a variety of GRFTmodified fibers against HIV-1 infection, certain process alterations may be considered to customize EF morphology or to improve GRFT (or other protein) conjugation efficiency or fiber yield ${ }^{75}$. In particular, the EF surface area may be increased by decreasing the fiber diameter, to enable a greater surface area for conjugation. Previous studies have shown that reducing polymer concentration and viscosity produces smaller fiber diameter ${ }^{403,404}$. However, this approach is limited by the formation of beaded fibers when the concentration is below the threshold value. To decrease fiber diameter without changing solution viscosity, dual-solvent systems can be utilized to reduce the surface tension, or salts may be added to increase solution conductivity 256,399 . Both methods will enable greater 
stretching of the electrospinning jets which may produce smaller fiber diameters. In addition, lower molecular weight polymers may be used to fabricate smaller diameter fibers. Decreasing fiber diameter also provides the added advantage of generating smaller pores, potentially making EFs more effective as a barrier to virus penetration, for microbicide-based applications ${ }^{405}$. Finally, it was observed that humidity can affect EF yield. At higher humidity, the yield tends to decrease due to the formation of fibers with unusual macro-morphology. Installing a humidity control system within the electrospinning chamber could therefore facilitate producing EFs with consistent yields, if this presents a challenge to fabrication.

To improve GRFT conjugation efficiency, investigating the selection and location of functionalizable groups may also be considered. For example, if the primary amines of the protein of interest are located near the interior of the threedimensional protein structure, steric hindrance may prevent activated carboxyl groups on EFs from interacting with primary amines, thereby decreasing the likelihood of the reaction. This challenge may be overcome by amino acid substitution of the protein to generate a primary amine group closer to the protein surface. However, as GRFT and other proteins depend on the specific activity of its binding sites for functionality, alterations in protein conformation should be thoroughly tested in functional assays prior to conjugation.

\section{Limitations}

A major limitation of GRFT surface modification is the potential for low conjugation efficiency using carbodiimide crosslinking chemistry. If the antiviral 
protein of interest has a high $\mathrm{IC}_{50}$, a low conjugation efficiency may not provide sufficient protection (in these applications) against virus infection. However, for GRFT (or other modified) EFs, proteins and active agents that are not covalently bound to EFs may still adsorb to the surface. These adsorbed agents offer the potential to complement the activity of conjugated GRFT, by transiently increasing the localized concentration available for virus binding. In this example, desorbed GRFT may bind to HIV-1 virions that do not directly contact EFs, and may provide an alternative mechanism of protection with the first $4 \mathrm{~h}$ of (pericoital) administration. Thus, both conjugated and surface-adsorbed GRFT may contribute to providing uniform protection against STIs.

Despite the protection conferred from both protein conjugation and desorption, other surface-modification strategies with potentially higher efficiency might be pursued. For example, PLGA terminated with amines instead of carboxyl groups could be used to conjugate activated GRFT. Alternatively, a different surface-modification strategy may be utilized to improve EF-protein conjugation efficiency. Nanofibrous membranes composed of EFs have been functionalized with avidin via carbodiimide crosslinking chemistry ${ }^{406}$. Biotinylation or addition of a Strep-tag (Trp-Ser-His-Pro-Gln-Phe-Glu-Lys) through recombination may enable the modified protein to form strong and extremely stable non-covalent interaction with avidin EFs. While non-covalent, the avidin-biotin linkage is the strongest noncovalent bond, with femtomolar affinity, likely resulting in stable conjugation to the fiber surface. For any surface-modification strategies, steric hindrance should be considered to maximize conjugation efficiency. 
Lastly, we envision that surface-modified EFs will offer an alternative strategy to active agent encapsulation to provide complementary modes of protection. One consideration with combining encapsulation and surfacemodification technologies on a single EF platform will be reducing the premature release of active agents during the surface-modification process. For surfacemodification reactions that require relatively long incubation time in aqueous solution, a significant percentage of the active agents loaded could be lost due to polymer hydrolysis.

\section{Significance of the Method with Respect to Existing and Alternative Methods}

In previous work, we observed that unmodified blank EFs were able to inhibit HIV-1 infection by $\sim 38 \%$ when placed in a transwell insert above infectible cells $^{75}$. This observation combined with the biocompatibility, web-like microstructure, and tortuosity of EFs, in parallel with the observed potent antiviral and adhesive properties of GRFT, prompted the development of the surfacemodified EFs described here.

Relative to other delivery technologies currently employed in microbicide applications, EFs have a wide range of potential applications due to their architecture and capacity for customization. In other work, the fibrous morphology of EFs has enabled them to deliver active agents, and to mimic the ECM, making them suitable scaffolds for tissue engineering. EFs have also been surfacemodified to enhance biocompatibility and enhance sustained-release ${ }^{407,408}$. 
To enhance biocompatibility or deliver agents that function through specific binding activity, numerous methods exist that allow for the attachment of compounds to the surfaces of EFs such as plasma treatments, wet chemical methods, and surface graft polymerizations ${ }^{362}$. In the case of GRFT which specifically bind to the viral glycoproteins of HIV-1, the wet chemical method of EDC-NHS is the most optimal due to the ability to penetrate fibers deep within the mesh while also preserving GRFT functionality ${ }^{362}$. GRFT immobilized to EFs can then be delivered in a durable formulation to the FRT and provide immediate protection against HIV-1 infection.

Compared to the existing strategy of encapsulating therapeutics within EFs to inhibit STIs, covalent conjugation offers the distinct advantage of increasing the potential avidity between GRFT and HIV-1 virions. By immobilizing GRFT to EFs through surface-modification, highly localized concentrations of GRFT can be achieved, which increase the opportunity for multivalent binding with HIV-1. In addition, EF-immobilized GRFT, relative to free GRFT in solution, may prevent the depletion of the GRFT pool by hindering cell internalization. Moreover, due to the unique mechanism of action of GRFT as a stable and adhesive entry inhibitor, covalent surface conjugation enables surface-modified EFs to provide a physical barrier to virus penetration, in addition to potent antiviral properties.

\section{Future Applications of this Method}

The utilization of surface-modification for delivery presents the opportunity to integrate multiple active agents within a single EF platform. In the future, we 
seek to develop a multipurpose technology (MPT) where a variety of active agents may be encapsulated within and conjugated to EFs. These MPTs may be designed to confer protection against a wider range of pathogens by incorporating therapeutics with different mechanisms of action. By utilizing both the surface and interior of EFs to deliver active agents with different mechanisms of action, the potential of EFs to protect against virus infection will be maximized. 
Target Product Profile

Table A 1. GRFT Electrospun Fibers Target Product Profile

\begin{tabular}{|c|c|c|}
\hline \multicolumn{3}{|c|}{ GRFT Electrospun Fibers Target Product Profile } \\
\hline Formulation Properties & $\begin{array}{c}\text { Minimum Acceptable } \\
\text { Result }\end{array}$ & Ideal Result \\
\hline Primary Purpose* & $\begin{array}{l}\text { To fabricate GRFT } \\
\text { encapsulated electrospun } \\
\text { fibers that will provide } \\
\text { sustained release of GRFT } \\
\text { for one week to one month. } \\
\text { Furthermore, the released } \\
\text { GRFT will provide } \\
\text { protection against both } \\
\text { HSV-2 and HIV-1 in vitro as } \\
\text { well as HSV-2 in vivo } \\
\text { against a single challenge. }\end{array}$ & $\begin{array}{l}\text { To fabricate GRFT } \\
\text { encapsulated electrospun } \\
\text { fibers that will provide } \\
\text { sustained release of GRFT } \\
\text { for up to one month. } \\
\text { Furthermore, the released } \\
\text { GRFT will provide complete } \\
\text { protection against both } \\
\text { HSV-2 and HIV- } 1 \text { in vitro as } \\
\text { well as HSV-2 in vivo } \\
\text { against multiple challenges. }\end{array}$ \\
\hline In Vitro Target Population & $\begin{array}{l}\text { TZM-bl, Vero, VK2, Ect, } \\
\text { End. }\end{array}$ & $\begin{array}{l}\text { TZM-bl, Vero, VK2, Ect, } \\
\text { End. }\end{array}$ \\
\hline In Vivo Target Population & BALB/c female mice. & BALB/c female mice. \\
\hline In Vitro Treatment Duration & $\begin{array}{l}\text { Test } 1 \text { day to } 1 \text { month } \\
\text { eluates. }\end{array}$ & $\begin{array}{l}\text { Test } 1 \text { day to } 1 \text { month } \\
\text { eluates. }\end{array}$ \\
\hline In Vivo Treatment Duration & Test single dose of fibers. & Test single dose of fibers. \\
\hline In Vivo Delivery Mode & $\begin{array}{l}\text { Intravaginal administration } \\
\text { for murine model. }\end{array}$ & $\begin{array}{l}\text { Intravaginal administration } \\
\text { for murine model. }\end{array}$ \\
\hline In Vivo Regimen & $\begin{array}{l}\text { One administration for the } \\
\text { entire duration. }\end{array}$ & $\begin{array}{l}\text { One administration for the } \\
\text { entire duration. }\end{array}$ \\
\hline Dosage for In Vitro & $\begin{array}{l}3-10 \mathrm{mg} \text { of GRFT fiber for in } \\
\text { vitro studies. Minimum } \\
\text { release is } 600 \mathrm{ng} / \mathrm{mL} \text { per } \\
\text { day against HIV }-1409,410 \text {, } \\
\text { while at least } 19-39 \mu \mathrm{g} / \mathrm{mL} \\
\text { is required for HSV- } 2^{65,71} \text {. }\end{array}$ & $\begin{array}{l}3-10 \mathrm{mg} \text { of GRFT fiber for in } \\
\text { vitro studies. Minimum } \\
\text { release is } 600 \mathrm{ng} / \mathrm{mL} \text { per } \\
\text { day against HIV-1409,410, } \\
\text { while at least } 19-39 \mu \mathrm{g} / \mathrm{mL} \\
\text { is required for HSV-2 } 2^{65,71} \text {. }\end{array}$ \\
\hline Dosage for In Vivo & $\begin{array}{l}4-5 \mathrm{mg} \text { of GRFT fiber for in } \\
\text { vivo studies. Minimum } \\
\text { release is } 600 \mathrm{ng} / \mathrm{mL} \text { per } \\
\text { day against HIV-1 } 1^{409,410} \text {, } \\
\text { while at least } 19-39 \mu \mathrm{g} / \mathrm{mL} \\
\text { is required for HSV- } 2^{65,71} \text {. }\end{array}$ & $\begin{array}{l}4-5 \mathrm{mg} \text { of GRFT fiber for in } \\
\text { vivo studies. Minimum } \\
\text { release is } 600 \mathrm{ng} / \mathrm{mL} \text { per } \\
\text { day against HIV-1 } 1^{409,410} \text {, } \\
\text { while at least } 19-39 \mu \mathrm{g} / \mathrm{mL} \\
\text { is required for HSV-2 }{ }^{65,71} \text {. }\end{array}$ \\
\hline In Vitro Efficacy & $\begin{array}{l}\text { Greater than } 90 \% \text { reduction } \\
\text { in } \mathrm{HIV}-1_{\text {BaL }} \text { infectivity for up } \\
\text { to } 1 \text { week eluates. } \\
50-90 \% \text { reduction of cell to } \\
\text { cell spread (via plaque } \\
\text { counts). }\end{array}$ & $\begin{array}{l}\text { Greater than } 90 \% \text { reduction } \\
\text { in HIV- } 1_{\text {BaL infectivity for up }} \\
\text { to } 1 \text { month eluates. } \\
\text { At least } 90 \% \text { reduction of } \\
\text { cell to cell spread (via } \\
\text { plaque counts). }\end{array}$ \\
\hline
\end{tabular}




\begin{tabular}{|c|c|c|}
\hline In Vivo Efficacy & $\begin{array}{l}\text { HSV-2: } 5,000 \text { PFU/20 } \mu \mathrm{L} \\
\left(\mathrm{LD}_{90}\right) \text {. Single dose of fibers } \\
\text { provides greater than } 90 \% \\
\text { protection for at least one } \\
\text { week. }\end{array}$ & $\begin{array}{l}\text { HSV-2: } 5,000 \text { PFU } / 20 \mu \mathrm{L} \\
\left(\mathrm{LD}_{90}\right) \text {. Single dose of fibers } \\
\text { provides greater than } 90 \% \\
\text { protection for for up to one } \\
\text { month. }\end{array}$ \\
\hline In Vitro Risk/Side Effects & $\begin{array}{l}\text { Greater than } 80 \% \text { viability } \\
\text { (MTT assay) in cell lines } \\
\text { after } 3 \text { day culture. }\end{array}$ & $\begin{array}{l}\text { Greater than } 80 \% \text { viability } \\
\text { (MTT assay) in cell lines } \\
\text { after } 3 \text { day culture. }\end{array}$ \\
\hline In Vivo Risk/Side Effects & $\begin{array}{l}\text { No histopathological } \\
\text { aberrations in the FRT from } \\
\text { exposure to GRFT fibers. } \\
\text { No more than a } 2-3 \text { fold } \\
\text { difference in cytokines } \\
\text { (GM-CSF, IFN- }- \text {, IL-1 } \alpha \text {, IL- } \\
1 \beta \text {, IL-6, CCL-2, TNF- } \alpha \text { ) } \\
\text { after exposure to GRFT } \\
\text { EFs }{ }^{68} \text {. }\end{array}$ & 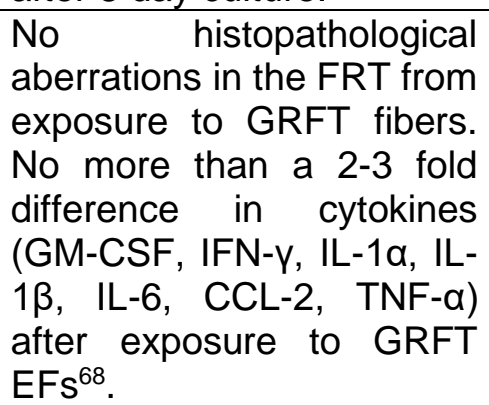 \\
\hline Stability & $\begin{array}{l}\text { Most (>50\%) incorporated } \\
\text { GRFT is stable for } 1 \text { month. }\end{array}$ & $\begin{array}{l}\text { All incorporated GRFT is } \\
\text { stable for } 1 \text { month. }\end{array}$ \\
\hline
\end{tabular}

\section{Primary Purpose}

\section{Minimum Acceptable Result}

The primary purpose of using polymeric electrospun fibers as delivery vehicles is to provide sustained-release of the antiviral Griffithsin (GRFT) for one month. Additionally, both the incorporated and released GRFT will maintain its bioactivity and by extension antiviral properties. Acceptable fiber formulations will release enough GRFT to significantly protect against both HIV-1 as well as HSV2 throughout a week's duration. Furthermore, GRFT release from fibers should continue for an entire month.

\section{Ideal Result}


The ideal result would meet all of the specifications listed above, releasing enough GRFT to completely prevent HIV-1 or HSV-2 infection for the entire one month duration.

\section{In Vitro Target Population}

\section{Minimum Acceptable Result}

These fibers will be assessed in vitro against both HIV-1 and HSV-2 infection. To assess against HIV-1 infection TZM-bl cell lines will be used in an HIV-1 inhibition assay. These cell lines are an accepted cell model to assess the therapeutic efficacy against HIV-1 infection. Eluates from GRFT EFs at various time points during the one month period will be evaluated.

For HSV-2 infection, plaque assays using Vero E6 cell lines will be used. Vero E6 is a widely used and accepted model to determine viral infection. For both HIV-1 and HSV-2 assays, the minimum acceptable result will be a significant decrease in infection within a 1 week duration. In addition, a decrease of cell-tocell spread will be assessed in our HSV-2 studies. Finally, VK2/E6E7, Ect1/E6E7, and End/E6E7, cell lines will be used to assess cytotoxicity. An acceptable result is that cells in contact with GRFT EFs exhibit $80-97 \%$ viability.

\section{Ideal Result}

The ideal result is that electrospun fibers completely inhibit HIV-1 and HSV2 infections while maintaining full viability of selected cell lines.

\section{In Vivo Target Population}




\section{Minimum Acceptable Result}

$\mathrm{BALB} / \mathrm{c}$ female mice will be used for these studies. This mouse type is an acceptable model for studies involving HSV-2 infection.

\section{Ideal Result}

Same as minimum acceptable result (MAR).

\section{In Vitro Treatment Duration}

\section{Minimum Acceptable Result}

Any fabricated fiber incorporating GRFT must release GRFT throughout the entire 1 month duration. However, the MAR is therapeutically relevant GRFT release during the first week of release.

\section{Ideal Result}

Any fabricated fiber incorporating GRFT must release enough of the antiviral to completely inhibit viral infection throughout the entire 1 month. Our ideal result is that therapeutically relevant concentrations of GRFT are released during the entire one month.

\section{In Vivo Treatment Duration}

\section{Minimum Acceptable Result}

Our in vivo studies are to be conducted for 1 month.

\section{Ideal Result}


Same as MAR.

\section{Delivery Mode}

\section{Minimum Acceptable Result}

Fibers will be delivered intravaginally to assess a new topical antiviral delivery vehicle that will be administrated within the female reproductive tract.

\section{Ideal Result}

Same as MAR.

\section{Regimen}

\section{Minimum Acceptable Result}

One administration for the entire 1 month duration.

\section{Ideal Result}

Same as MAR.

\section{In Vitro Dosage}

\section{Minimum Acceptable Result}

For GRFT released from fibers, a daily release of $600 \mathrm{ng} / \mathrm{mL}$ is required to provide an $\mathrm{IC}_{90}$ against HIV-1 infection ${ }^{409,410}$. In regard to HSV-2 infection, literature mentions several $I_{50}$ values for GRFT dependent on cell type (19, 4.5, and $72 \mu \mathrm{g} / \mathrm{mL}$ for Vero $E 6^{71}$, Caski ${ }^{65}$, and VK2/E6E7 ${ }^{65}$ respectively). However, GRFT mainly acts to prevent the cell-to-cell spread of HSV-2 infection with $\mathrm{IC}_{50}$ 
values of 4.5 and $72 \mu \mathrm{g} / \mathrm{mL}^{65}$. As for IC90, GRFT concentrations of 39 and 335 $\mu \mathrm{g} / \mathrm{mL}$ were required to prevent of cell-to-cell spread ${ }^{65}$. Thus, for HSV-2, a GRFT daily release of at least $19-39 \mu \mathrm{g} / \mathrm{mL}$ will be required in our in vitro studies using Vero cell lines.

This release should also be enough to significantly decrease HIV-1 infection. To provide an $\mathrm{IC}_{90}$ against HIV-1 for one entire week, a fiber will have to release a total of at least $4.2 \mu \mathrm{g} / \mathrm{mL}$ of GRFT in total. For in vitro testing, a $3 \mathrm{mg}$ fiber sample with a loading of $1.4 \mu \mathrm{g} / \mathrm{mg}$ is needed to provide an IC 90 against HIV1 infection for 1 week (assuming complete release during this period). For HSV-2, to provide at least an $\mathrm{EC}_{50}$ for one week in Vero cells, a $3 \mathrm{mg}$ fiber sample must have a loading of $45 \mu \mathrm{g} / \mathrm{mg}(135 \mu \mathrm{g})$ of GRFT. To provide an IC 90 against HSV-2, a $3 \mathrm{mg}$ sample must load $91 \mu \mathrm{g} / \mathrm{mg}(273 \mu \mathrm{g})$ of GRFT.

\section{Ideal Result}

For GRFT released from fibers, we anticipate a daily release of $600 \mathrm{ng} / \mathrm{mL}$ for 1 month duration to provide an IC90 against HIV-1. This would require a fiber to release a total of $18 \mu \mathrm{g}$ of GRFT during this duration. For a $3 \mathrm{mg}$ fiber sample, the total loading would require a loading of at least $6 \mu \mathrm{g} / \mathrm{mg}$ (assuming full release after one month). As for HSV-2, $570 \mu \mathrm{g}$ of GRFT would need to be released to provide an $\mathrm{IC}_{50}$ against HSV-2 for one month, requiring a loading of $(190 \mu \mathrm{g} / \mathrm{mg})$ from a $3 \mathrm{mg}$ EF sample. To provide an $\mathrm{IC}_{90}$ against HSV-2 the entire one month duration, a $3 \mathrm{mg}$ EF sample must load $390 \mu \mathrm{g} / \mathrm{mg}(1.17 \mathrm{mg})$ of GRFT.

\section{In Vivo Dosage}




\section{Minimum Acceptable Result}

For GRFT released from fibers, a daily release of $1 \mu \mathrm{g} / \mu \mathrm{L}(0.1 \% \mathrm{wt} / \mathrm{vol})$ would be required to provide protection against HSV-2 within a mouse model ${ }^{65}$. This daily release must be extended for at least one week.

\section{Ideal Result}

For GRFT released from fibers, we anticipate a daily release of $3 \mu \mathrm{g} / \mu \mathrm{L}$ $(0.3 \% \mathrm{wt} / \mathrm{vol})$ for 1 month duration to provide an $\mathrm{EC}_{90}$ against HSV-2 infection within a mouse model. This would require a fiber to release a total of $1.8 \mathrm{mg}$ of GRFT during this duration. For a $10 \mathrm{mg}$ fiber sample, the total loading would require a loading of at least $180 \mu \mathrm{g} / \mathrm{mg}$ (assuming full release after one month).

\section{In Vitro Efficacy}

\section{Minimum Acceptable Result}

For HIV-1 in vitro testing, the minimal result acceptable would be at least a $90 \%$ decrease of infection during a 1 week period. In regard to HSV-2, a decrease in plaque formation is ideal; however, a $50-90 \%$ reduction in cell-to-cell spread of HSV-2 is required.

\section{Ideal Result:}

The ideal result would be that collected daily or weekly eluates collected from fibers demonstrate a $90 \%$ decrease of both HIV-1 and HSV-2 infections. These eluates will be collected for up to one month. 


\section{In Vivo Efficacy}

\section{Minimum Acceptable Result}

Fibers provide $90 \%$ protection against a single challenge with HSV-2 (LD90) for 1 week.

\section{Ideal Result}

Fibers provide $90 \%$ protection against a single challenge with HSV-2 (LD90) for 1 month.

\section{In Vitro Risk/Side Effects}

\section{Minimum Acceptable Result}

An acceptable result would be cells in contact with GRFT EFs exhibiting 70$100 \%$ viability. These viability values are from the JFHE grant proposal.

\section{Ideal Result}

Ideally, we would want no cytotoxicity expressed from contact from fibers.

\section{In Vivo Risk/Side Effects}

\section{Minimum Acceptable Result:}

From literature, GRFT is biocompatible and does not induce inflammation ${ }^{68}$. Furthermore, our selected polymers share the same attributes in safety. Therefore, the administration of GRFT fibers should not induce toxicity or lead to increased 
cytokine expression. Histopathological grading should reflect a lack of inflammation.

Ideal Result

Same as MAR.

\section{Stability}

\section{Minimum Acceptable Result}

GRFT has shown remarkable stability ${ }^{409}$. Therefore, there should not be any decrease of activity shown form released GRFT. We predict that encapsulated GRFT will retain activity for the entire 1 month duration. However, since the amount of GRFT encapsulated within fibers will be more than necessary to provide IC90, a decrease of activity can be tolerated. For our purposes, a decrease of at most $50 \%$ will be tolerated.

\section{Ideal Result}

Ideally, we would want all incorporated and released GRFT to maintain bioactivity for the entire duration. 
Table A 2. GRFT Nanoparticle Target Product Profile

\begin{tabular}{|c|c|c|}
\hline \multicolumn{3}{|c|}{ GRFT Nanoparticle Target Product Profile } \\
\hline Formulation Properties & $\begin{array}{c}\text { Minimum Acceptable } \\
\text { Result }\end{array}$ & Ideal Result \\
\hline Primary Purpose* & $\begin{array}{l}\text { To fabricate encapsulated } \\
\text { GRFT NPs that will provide } \\
\text { sustained release of GRFT } \\
\text { for up to one month. } \\
\text { Furthermore, the released } \\
\text { GRFT and will provide } \\
\text { significant protection } \\
\text { against both HSV-2 and } \\
\text { HIV-1 in vitro as well as } \\
\text { HSV-2 in vivo. }\end{array}$ & $\begin{array}{l}\text { To fabricate encapsulating } \\
\text { GRFT NPs that will provide } \\
\text { sustained release of GRFT } \\
\text { for up to one month. } \\
\text { Furthermore, the released } \\
\text { GRFT and will provide } \\
\text { complete significant } \\
\text { protection against both } \\
\text { HSV-2 and HIV-1 in vitro as } \\
\text { well as HSV-2 in vivo. }\end{array}$ \\
\hline In Vitro Target Population & $\begin{array}{l}\text { TZM-bl, Vero, VK2, Ect, } \\
\text { End. }\end{array}$ & $\begin{array}{l}\text { TZM-bl, Vero, VK2, Ect, } \\
\text { End. }\end{array}$ \\
\hline In Vivo Target Population & BALB/c female mice. & BALB/c female mice. \\
\hline In Vitro Treatment Duration & $\begin{array}{l}\text { Test } 1 \text { day to } 1 \text { month } \\
\text { eluates. }\end{array}$ & $\begin{array}{l}\text { Test } 1 \text { day to } 1 \text { month } \\
\text { eluates. }\end{array}$ \\
\hline In Vivo Treatment Duration & Test single dose of NPs. & Test single dose of NPs. \\
\hline Delivery Mode & $\begin{array}{l}\text { Intravaginal administration } \\
\text { for murine model. }\end{array}$ & $\begin{array}{l}\text { Intravaginal administration } \\
\text { for murine model. }\end{array}$ \\
\hline Regimen & $\begin{array}{l}\text { One administration for the } \\
\text { entire duration. }\end{array}$ & $\begin{array}{l}\text { One administration for the } \\
\text { entire duration. }\end{array}$ \\
\hline Dosage for In Vitro & $\begin{array}{l}2-5 \mathrm{mg} \text { for in vitro studies. } \\
\text { Minimum release is } 600 \\
\mathrm{ng} / \mathrm{mL} \text { per day against HIV- } \\
1^{409,410} \text {, while at least } 19-39 \\
\mu \mathrm{g} / \mathrm{mL} \text { is required for HSV- } \\
65,71 \text {. }\end{array}$ & $\begin{array}{l}2-5 \mathrm{mg} \text { for in vitro studies. } \\
\text { Minimum release is } 600 \\
\mathrm{ng} / \mathrm{mL} \text { per day against HIV- } \\
1^{409,410} \text {, while at least } 19-39 \\
\mu \mathrm{g} / \mathrm{mL} \text { is required for HSV- } \\
2^{65,71} \text {. }\end{array}$ \\
\hline Dosage for In Vivo & $\begin{array}{l}4 \mathrm{mg} \text { in } 20 \mu \mathrm{L} \text { for in vivo } \\
\text { studies. Minimum release } \\
\text { is } 600 \mathrm{ng} / \mathrm{mL} \text { per day } \\
\text { against } \mathrm{HIV} 409,410 \text {, while at } \\
\text { least } 19-39 \mu \mathrm{gg} / \mathrm{mL} \text { is } \\
\text { required for HSV-2 } \\
\text { res,71. }\end{array}$ & $\begin{array}{l}4 \mathrm{mg} \text { in } 20 \mu \mathrm{L} \text { for in vivo } \\
\text { studies. Minimum release } \\
\text { is } 600 \mathrm{ng} / \mathrm{mL} \text { per day } \\
\text { against } \mathrm{HIV} \mathrm{V}^{409,410} \text {, while at } \\
\text { least } 19-39 \mu \mathrm{g} / \mathrm{mL} \text { is } \\
\text { required for HSV-2 }{ }^{65,71} \text {. }\end{array}$ \\
\hline In Vitro Efficacy & $\begin{array}{l}\text { Greater than } 90 \% \text { reduction } \\
\text { in HIV- } 1_{\text {BaL infectivity for up }} \\
\text { to } 1 \text { wk eluates. } \\
50-90 \% \text { reduction of cell to } \\
\text { cell spread (via plaque } \\
\text { counts). }\end{array}$ & $\begin{array}{l}\text { Greater than } 90 \% \text { reduction } \\
\text { in HIV- } 1_{\mathrm{BaL}} \text { infectivity for up } \\
\text { to } 1 \text { month eluates. } \\
\text { At least } 90 \% \text { reduction of } \\
\text { cell to cell spread (via } \\
\text { plaque counts). }\end{array}$ \\
\hline In Vivo Efficacy & $\begin{array}{l}\text { HSV-2: } 5,000 \text { PFU/20 } \mu \mathrm{L} \\
\left(\mathrm{LD}_{90}\right) \text {. Single dose of NPs } \\
\text { provides greater than } 90 \% \\
\text { protection for at least one } \\
\text { week. }\end{array}$ & $\begin{array}{l}\text { HSV-2: } 5,000 \text { PFU/ } 20 \mu \mathrm{L} \\
\left(\mathrm{LD}_{90}\right) \text {. Single dose of } \\
\text { provides greater than } 90 \% \\
\text { protection for at least one } \\
\text { month. }\end{array}$ \\
\hline
\end{tabular}




\begin{tabular}{|c|c|c|}
\hline In Vitro Risk/Side Effects & $\begin{array}{l}\text { Greater than } 80 \% \text { viability } \\
\text { (MTT assay) in cell lines } \\
\text { after } 3 \text { day culture. }\end{array}$ & $\begin{array}{l}\text { Greater than } 80 \% \text { viability } \\
\text { (MTT assay) in cell lines } \\
\text { after } 3 \text { day culture. }\end{array}$ \\
\hline In vivo Risk/Side Effects & 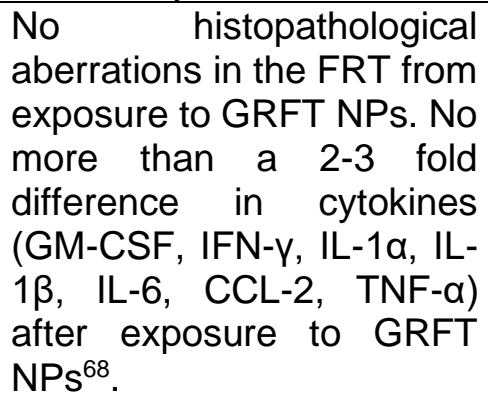 & $\begin{array}{l}\text { No histopathological } \\
\text { aberrations in the FRT from } \\
\text { exposure to GRFT NPs. No } \\
\text { more than a } 2-3 \text { fold } \\
\text { difference in cytokines } \\
\text { (GM-CSF, IFN- }- \text {, IL-1 } \alpha \text {, IL- } \\
1 \beta \text {, IL-6, CCL-2, TNF- } \alpha \text { ) } \\
\text { after exposure to GRFT } \\
\text { NPs }{ }^{68} \text {. }\end{array}$ \\
\hline Stability & $\begin{array}{l}\text { Most (>50\%) incorporated } \\
\text { GRFT is stable for } 1 \text { month. }\end{array}$ & $\begin{array}{l}\text { All incorporated GRFT is } \\
\text { stable for } 1 \text { month. }\end{array}$ \\
\hline
\end{tabular}

\section{Primary Purpose}

\section{Minimum Acceptable Result}

The primary purpose of using polymeric nanoparticles (NPs) as mobile delivery vehicles is to provide sustained-release of the antiviral griffithsin (GRFT) for one month. Additionally, both the incorporated and released GRFT will maintain its bioactivity and by extension antiviral properties. Acceptable NP formulations will release enough GRFT to significantly protect against both HIV-1 as well as HSV2 throughout a week's duration. Furthermore, GRFT release from NPs should continue for an entire month.

\section{Ideal Result}

The ideal result would meet all of the specifications listed above, releasing enough GRFT to completely prevent HIV-1 or HSV-2 infection for the entire one month duration

\section{In Vitro Target Population}




\section{Minimum Acceptable Result}

These NPs will be assessed in vitro against both HIV-1 and HSV-2 infection. To assess against HIV-1 infection TZM-bl cell lines will be used in an HIV-1 inhibition assay. These cell lines are an accepted cell model to assess the therapeutic efficacy against HIV-1 infection. Eluates from GRFT NPs at various time points during the one month period will be evaluated.

For HSV-2 infection, plaque assays using Vero E6 cell lines will be used. Vero E6 is a widely used and accepted model to determine viral infection. For both HIV-1 and HSV-2 assays, the minimal acceptable result will significantly decrease in infection within a 1 week period. A decrease of cell-to-cell spread will be assessed during the HSV-2 studies. Finally, VK2/E6E7, Ect1/E6E7, and End/E6E7 cell lines will be used to assess cytotoxicity. An acceptable result is that cells in contact with GRFT NPs exhibit 80-97\% viability.

\section{Ideal Result}

The ideal result is that NPs completely inhibit HIV-1 and HSV-2 infections while maintaining full viability of selected cell lines.

\section{In Vivo Target Population}

\section{Minimum Acceptable Result}

$\mathrm{BALB} / \mathrm{c}$ female mice will be used for these studies. This mouse type is an acceptable model for STI studies.

\section{Ideal Result}


Same as minimum acceptable result (MAR).

\section{In Vitro Treatment Duration}

\section{Minimum Acceptable Result}

NPs incorporating GRFT must release GRFT throughout the entire 1 month.

\section{Ideal Result}

GRFT NPs release enough of the antiviral to completely inhibit viral infection throughout the entire 1 month.

\section{In Vivo Treatment Duration}

\section{Minimum Acceptable Result}

Our in vivo studies are to be conducted for 1 month.

\section{Ideal Result}

Same as MAR.

\section{Delivery Mode}

\section{Minimum Acceptable Result}

NPs will be delivered intravaginally using PBS to assess a new topical antiviral delivery vehicle that will be administrated within the female reproductive tract.

Ideal Result 
Same as MAR.

\section{Regimen}

\section{Minimum Acceptable Result}

One administration for the entire 1 month duration.

\section{Ideal Result}

Same as MAR.

\section{In Vitro Dosage}

\section{Minimum Acceptable Result}

For GRFT released from nanoparticles, a daily release of $600 \mathrm{ng} / \mathrm{mL}$ is required to provide an $\mathrm{IC}_{90}$ against HIV-1 infection. As for HSV-2 infection, a daily release of $19-39 \mu \mathrm{g} / \mathrm{mL}$ would be required to provide an $\mathrm{EC}_{50}$ for Vero cell lines ${ }^{71}$. This release should also be enough to significantly decrease HIV-1 infection. To provide an IC90 against HIV-1 for one entire week, NPs will have to release a total of at least $4.2 \mu \mathrm{g} / \mathrm{mL}$ of GRFT for one week. For in vitro testing, a $2 \mathrm{mg}$ with a loading of $2.1 \mu \mathrm{g} / \mathrm{mg}$ is needed provide an IC90 against HIV-1 infection for 1 week (assuming complete release during this period). For HSV-2, to provide at least an

$\mathrm{EC}_{50}$ for one week, a $2 \mathrm{mg}$ of NPs must have a loading of $66 \mu \mathrm{g} / \mathrm{mg}(135 \mu \mathrm{g})$ of GRFT. To provide an IC90 against HSV-2, a 2 mg NP sample must load $136 \mu \mathrm{g} / \mathrm{mg}$ $(273 \mu \mathrm{g})$ of GRFT.

\section{Ideal Result}


For GRFT released from nanoparticles, we anticipate a daily release of 600 $\mathrm{ng} / \mathrm{mL}$ for 1 month duration to provide an IC 90 against HIV-1. This would require NPs to release a total of $18 \mu \mathrm{g}$ of GRFT during this duration. For a $2 \mathrm{mg}$ NP sample, the total loading would require a loading of at least $9 \mu \mathrm{g} / \mathrm{mg}$ (assuming full release after one month). As for HSV-2, $570 \mu \mathrm{g}$ of GRFT would need to be released to provide an $\mathrm{IC}_{50}$ against HSV-2 for one month, requiring a loading of $(285 \mu \mathrm{g} / \mathrm{mg})$ from a $2 \mathrm{mg}$ NP sample. To provide an $\mathrm{IC}_{90}$ against HSV-2 the entire one month duration, a $2 \mathrm{mg} \mathrm{NP}$ sample must load $585 \mu \mathrm{g} / \mathrm{mg}(1.17 \mathrm{mg})$ of GRFT.

\section{In Vivo Dosage}

\section{Minimum Acceptable Result}

For GRFT released from NPs, a daily release of $1 \mu \mathrm{g} / \mu \mathrm{L}(0.1 \% \mathrm{wt} / \mathrm{vol})$ would be required to provide protection against HSV-2 within a mouse model ${ }^{65}$. This daily release must be extended for at least one week.

\section{Ideal Result}

For GRFT released from NPs, we anticipate a daily release of $3 \mu \mathrm{g} / \mu \mathrm{L}(0.3 \%$ wt/vol) for 1 month duration to provide an $\mathrm{EC}_{90}$ against HSV-2 infection within a mouse model. This would require a fiber to release a total of $1.8 \mathrm{mg}$ of GRFT during this duration. For a $4 \mathrm{mg} \mathrm{NP}$ sample, the total loading would require a loading of at least $450 \mu \mathrm{g} / \mathrm{mg}$ (assuming full release after one month).

\section{In Vitro Efficacy}

\section{Minimum Acceptable Result}


For HIV-1 in vitro testing, the minimal result acceptable would be at least a $90 \%$ decrease of infection during a 1 week period. In regard to HSV-2, a decrease in plaque formation is ideal; however, a $50-90 \%$ reduction in cell-to cell-spread of HSV-2 is required.

\section{Ideal Result}

The ideal result would be that daily or weekly eluates collected from GRFT NPs demonstrate a $90 \%$ decrease of both HIV-1 and HSV-2 infections. These eluates will be collected for up to one month.

\section{In Vivo Efficacy}

\section{Minimum Acceptable Result}

GRFT NPs provide $90 \%$ protection with a single challenge against HSV-2 (LDgo) for 1 week.

\section{Ideal Result}

GRFT NPs provide $90 \%$ protection against a single challenge with HSV-2 $\left(\mathrm{LD}_{90}\right)$ for 1 month.

\section{In Vitro Risk/Side Effects}

\section{Minimum Acceptable Result}

An acceptable result would be cells in contact with GRFT NPs exhibiting $80-100 \%$ viability. 
Ideal Result

Ideally, we would want no cytotoxicity expressed from contact from nanoparticles.

In vivo Risk/Side Effects

Minimum Acceptable Result

Same as EFs.

Ideal Result

Same as EFs.

Stability

Minimum Acceptable Result

Same as EFs.

Ideal Result

Ideally, we would want all incorporated and released GRFT to maintain bioactivity. 


\section{LIST OF ABBREVIATIONS}

ACV - Acyclovir

AIDS - Acquired immunodeficiency syndrome

ARRRP - AIDS Research and Reference Reagent Program

AZT - Azidothymidine

BSA - Bovine serum albumin

C6 - Coumarin 6

CCR5 - Chemokine receptor 5

CD4 - Cluster of differentiation 4 receptor

CG - Carrageenan

CXCR4 - Chemokine receptor type 4

D - Day

DCM - Dichloromethane

DMEM - Dulbecco`s modified Eagle media

DMSO - Dimethyl sulfoxide

EC $90-90 \%$ maximal response

ECM - Extracellular matrix

EDC - 1-ethyl-3-(3-dimethylaminopropyl)carbodiimide hydrochloride

EE\% - Encapsulation efficiency

EFs - Electrospun fibers 
ELISA - Enzyme-linked immunosorbent assay

ENV - Envelope glycoprotein

FBS - Fetal bovine serum

FDA - Food and Drug Administration

FDI - Fast dissolving insert

FRT - Female reproductive tract

GRFT - Griffithsin

HAART - Highly active antiretroviral therapy

H\&E - Haemotoxylin and Eosin

HFIP - Hexafluoro-2-propanol

HIV-1 - Human immunodeficiency virus

HSV-2 - Herpes simplex virus

$\mathrm{IC}_{50}$ - The half maximal inhibitory concentration

IVR - Intravaginal ring

KFSM - Keratinocyte serum-free media

LC/MS - Liquid chromatography-mass spectrometry

LD $_{10}$ - Lethal dose $10 \%$

LD90 - Lethal dose $90 \%$

MEM - Minimal essential medium

MES - 2-ethanesulfonic acid

mPEG-PLGA - methoxy poly(ethylene glycol)-b-poly(lactide-co-glycolide)

MTT - 3-(4,5-dimethylthiazol-2-yl)-2,5-diphenyltetrazolium bromide N-9 - Nonoxynol-9 
$\mathrm{NHS}-\mathrm{N}$-Hydroxysuccinimide

$\mathrm{NIH}$ - National Institutes of Health

NNRTIs - Non-nucleoside/nucleotide reverse transcriptase inhibitors

NP-EFs - Nanoparticle-Electrospun Fiber composites

NPs - Nanoparticles

NRTI - Nucleoside/nucleotide reverse transcriptase inhibitors

PAA - Poly(acrylic acid)

PBA - Poly(n-butyl acrylate)

PBS - Phosphate-buffered saline

PBS-T - Phosphate-buffered saline Tween 20

PCL - Polycaprolactone

PEO - Poly(ethylene oxide)

PFU - Plaque forming units

$\mathrm{PI}$ - Protease inhibitors

PLCL - Poly(lactide-co-caprolactone)

PLGA - Poly(lactic-co-glycolic acid)

PLLA - Polylactide (Poly(lactic acid)

PrEP - Pre-exposure prophylaxis

PVA - Polyvinyl alcohol

PVP - Polyvinylpyrrolidone

RhB - Rhodamine B

RLU - Relative luminescence unit

SDS - sodium dodecyl sulfate 
SEM - Scanning electron microscopy

SiRNA - Small interfering RNA

SIV - Simian immunodeficiency virus

STI - Sexually transmitted infection

SSF - simulated semen fluid

SVF - Simulated vaginal fluid

$\mathrm{TCID}_{50}$ - Median tissue culture infectious dose $50 \%$

TEER - Transepithelial/transendothelial electrical resistance

TFE - 2,2,2 -Trifluoroethanol

TDF -Tenofovir disoproxil fumarate

TFV - Tenofovir

WK - Week 


\section{CURRICULUM VITAE}

Kevin M. Tyo

Phone: (502) 424-8625

Email: kevinmtyo@gmail.com

Address: 901 Willow Springs Drive, Apt. A,

Louisville, KY 40242

\section{Professional Summary}

Current doctoral candidate of Pharmacology and Toxicology from The University of Louisville with over 10 years of laboratory experience.

\section{EDUCATION}

Ph.D. University of Louisville, Louisville, KY

Pharmacology and Toxicology

Expected Dec 2019

Dissertation: "Electrospun Fibers and Nanoparticles for The

Prevention of Sexually Transmitted Infections."

Advisor: Dr. Jill Steinbach-Rankins

MS University of Louisville, Louisville, KY

Pharmacology and Toxicology

May 2016

Thesis: "Multipurpose Tenofovir Disoproxil Fumarate Electrospun

Fibers for the Prevention of HIV-1 and HSV-2 Infections."

Advisor: Dr. Jill Steinbach-Rankins

BS Virginia Polytechnic Institute and State University, Blacksburg, VA

Biochemistry

May 2010

\section{GraduAte Research ExPERIENCE}

University of Louisville, Louisville, KY

Aug 2014-Aug 2019

Ph.D. Candidate, Pharmacology and Toxicology, Center of Predictive Medicine

- Fabricated alternative topical delivery platforms encapsulating the novel protein Griffithsin for the prevention of sexually transmitted infections such as HIV-1 and HSV-2 within the female reproductive tract.

- Volunteered to supervise inventory management, item purchasing, hazardous waste compliance, and oversee personnel training.

Additionally, ssisted in mentoring and training students to perform a variety of lab experiments.

- Lead a small group to update laboratory standard operating procedures. Within a two-month period, dozens of protocols were revised to more streamline training as well as to provide easier material purchasing. 
Arista Laboratories, Richmond, VA Position, Associate Scientist

Apr 2012-May 2014

- Performed extraction and analysis of target compounds by employing GC instrumentation. In charge of performing daily and preventative maintenance on instruments. Collaborated with personal on completing tasks within a GLP compliant laboratory.

- Managed a small team in a fast-paced environment to execute timely analysis of contract samples.

- Isolated selected compounds from difficult matrixes such as tobacco smoke and plant fiber.

- Assisted Methods Development in improving in house methods. Was able to consolidate the analysis of seven separate compounds, reducing the number of protocols from three into just one.

SLGI, Richmond, VA

Position, Laboratory Technician and Safety Officer

Mar 2011-Apr 2012

- Worked, trained, and troubleshot a variety of equipment including HPLC/UV-vis, HPLC-conductivity, GC/MS, GC/ECD, GC/FID, QuickChem 8500, and spectrophotometers.

- Proficient in the extraction and analysis of organic compounds in the environmental, industrial hygiene, and wet chemistry fields.

- Volunteered to assist in feasibility studies, quality control, and the creation of standard operating procedures for new analysis methods. Within a sixmonth period, the laboratory became certified for drinking water analysis, resulting in the acquisition of more impactful and lucrative contracts.

- Was personally requested for the position of safety officer by the owner of the company. During afterhours period, I streamlined safety training and created a more efficient transfer of safety-related information.

\section{UNDERGRADUATE RESEARCH EXPERIENCE}

Virginia Polytechnic Institute, Blacksburg, VA

Aug 2007-Jan 2008

Undergraduate Research, Biochemistry

- Produced research on mycothiol synthase proteins found in Tuberculosis Bacteria in order to explore new routes for antibiotics.

- Assisted in training, inventory management, lab cleanup, and stock solution preparations. Proficiency in bacterial cell culture for a variety of applications. Manufactured transgenic bacteria cultures for use in protein or DNA production. Maintained and assessed purity of products from bacterial cultures.

Virginia Polytechnic Institute, Blacksburg, VA Undergraduate Research, Biology 
- Research on Shigella Pseudomonas' proteins to better understand its virulence.

- Duties included protein expression, isolation and quantization, and plasmid preparation.

\section{Publications}

\section{Publications:}

Kevin M. Tyo, Amanda Lasnik, Longyun Zhang, Mohamed Mahmoud, Kenneth E. Palmer, Jill M. Steinbach-Rankins (In preparation). "Multipurpose Griffithsin nanoparticle-electrospun fiber composites against HIV-1 and HSV-2 infections."

Kevin M. Tyo, Amanda Lasnik, Longyun Zhang, Mohamed Mahmoud, Kenneth E. Palmer, Jill M. Steinbach-Rankins (In preparation). "Rapid Release Griffithsin Electrospun Fibers for Use Against Sexually Transmitted Infections."

Kevin M. Tyo*, Farnaz Minooei*, Keegan C. Curry, Sarah M. NeCamp, Danielle L. Graves, Joel R. Fried, and Jill M. Steinbach-Rankins (2019). "Relating Advanced Electrospun Fiber Architectures to the Temporal Release of Active Agents to Meet the Needs of Next-Generation Intravaginal Delivery Applications," Pharmaceutics 2019, 11(4), 160; https://doi.org/10.3390/pharmaceutics11040160.

Lee B. Sims; Kevin M. Tyo; Sanaya Stocke; Mohamed Y. Mahmoud; Aparna Ramasubramanian; Jill M. Steinbach-Rankins (2019) "Surface-Modified Melphalan Nanoparticles for Intravitreal Chemotherapy of Retinoblastoma," Investigative Ophthalmology \& Visual Science April 2019, Vol.60, 1696-1705. doi:10.1167/iovs.18-26251.

Kevin M. Tyo*, Jinghua Duan*, Pravallika Kollipara, Kenneth E. Palmer, Jill M. Steinbach-Rankins (2018). "pH-Responsive Delivery of Griffithsin from Electrospun Fibers", European Journal of Pharmaceutics, (138) 64-74 https://doi.org/10.1016/j.ejpb.2018.04.013.

Michael E. Halwes, Kevin M. Tyo, Jill M. Steinbach-Rankins§, Hermann B. Frieboes§ (2018). "Computational Modeling of PLGA Fiber Degradation and Resultant Multicompartment Tenofovir Pharmacokinetics in the Female Reproductive Tract", Molecular Pharmaceutics, 15(4): 1534-47, doi:10.1021/acs.molpharmaceut.7b01089.

Hung R. Vuong*, Kevin M. Tyo*, Jill M. Steinbach-Rankins (2017). "Fabrication and Characterization of Griffithsin-Modified Fiber Scaffolds for STI Prevention", Journal of Visual Experiments, (128), e56492, doi:10.3791/56492. 
Kevin M. Tyo, Hung R. Vuong, Danial A. Malik, Lee B. Sims, Houda Alatassi, Jinghua Duan, Walter H. Watson, Jill M. Steinbach-Rankins (2017).

"Multipurpose Tenofovir Disoproxil Fumarate Electrospun Fibers for the Prevention of HIV-1 and HSV-2 Infections", Intnl. Jnl. of Pharmaceutics, 531(1):118-133, doi: 10.1016/j.jpharm.2017.08.061.

Stella E. Aniagyei, Lee B. Sims, Danial A. Malik, Kevin M. Tyo, Keegan C. Curry, Woihwan Kim, Daniel A. Hodge, Jinghua Duan, Jill M. Steinbach-Rankins (2017). "Evaluation of Poly(Lactic-co-Glycolic Acid) and Poly(DL-Lactide-coCaprolactone) Electrospun Fibers for the Treatment of HSV-2 Infection", Materials Science and Engineering C, 1(72C):238-51, doi:

10.1016/j.msec.2016.11.029.

Tiffany N. Grooms, Hung R. Vuong, Kevin M. Tyo, Danial A. Malik, Lee B. Sims, Kenneth E. Palmer, Nobuyuki Matoba, Jill M. Steinbach-Rankins (2016). "Griffithsin-Modified Electrospun Fibers as a Delivery Scaffold to Prevent HIV Infection", Antimicrobial Agents and Chemotherapy, 60(11): 6518-31, doi: 10.1128/AAC.00956-16.

*Shared first authorship

\section{Presentations and Invited Lectures}

\section{Poster Presentations}

'Safety and efficacy of multilayered polymeric nanoparticle-electrospun fiber composites incorporating Griffithsin for use against HSV-2 infection' Controlled Release Society Annual Meeting \& Exposition Valencia, Spain, July 21-24, 2019.

'Rapid-release Griffithsin fibers provide in vivo protection against HSV-2 infection' Controlled Release Society Annual Meeting \& Exposition Valencia, Spain, July 21-24, 2019.

'Rapid Release Griffithsin Fibers for Use Against Sexually Transmitted Infections' Biomedical Engineering Society (BMES) Annual Meeting Atlanta, Georgia, October 17-20, 2018.

'Rapid Release Griffithsin Fibers for Use Against Sexually Transmitted Infections' Research! Louisville Louisville, Kentucky, October 9-11, 2018.

'Griffithsin based nanocarriers for the prevention of viral infections' Research! Louisville Louisville, Kentucky, September 11-15, 2017. 
'Electrospun Polymer Nanofibers for Long-term Protection Against HIV and HSV''

HIV Research for Prevention (HIVR4P) Annual Conference

Chicago, Illinois, October 17-20 2016.

'Electrospun Polymeric Fibers for Long-Term Protection against HIV and HSV-2' Research! Louisville

Louisville, Kentucky, October 11-14, 2016.

'Electrospun Nanofibers as a Novel Drug Delivery System for the Prevention of STIs'

Research! Louisville

Louisville, Kentucky, October 27-30, 2015.

\section{Oral Presentations}

'Review of Electrospun Polymeric Fibers and Advanced Architectures for Topical Drug Delivery Applications.'

University of Louisville, Department of Pharmacology and Toxicology

Louisville, Kentucky, June 27, 2019.

'Electrospun Polymer Nanofibers as a Drug Delivery Vehicle'

Center for Predictive Medicine Annual Retreat

Henryville, Indiana, November 19-20, 2015.

'A Multipurpose Prevention Technology or "Virus Trap and Safety Net" for the

Delivery of Antivirals, Proteins, and Oligonucleotides against STIs'

Biomedical Engineering Society (BMES) Annual Meeting

Tampa, Florida, October 7-10, 2015.

\section{Professional SkiLLS}

-Possesses practical experience in providing training and personnel mentoring.

-Broad expertise in scientific writing and communication skills.

- Experience in planning and implementing murine studies.

-Extensive practice designing, fabricating, characterizing delivery platforms such nanoparticles and polymeric electrospun fibers.

- Expertise in a variety of eukaryotic cell culture.

-Proficiency in bacterial cell culture for a variety of applications.

-Wide expertise in the field of analytical chemistry.

\section{Professional Affiliations}

Member of the Bioengineering Society, 2015-Present 
Member of the Controlled Release Society, 2019-Present 